Maximilian Eichhorn

Logistische Entscheidungen und ihre Auswirkungen:

Die Unternehmenssimulation LogisticPLUS 


\title{
Logistische Entscheidungen und ihre Auswirkungen: Die Unternehmenssimulation LogisticPLUS
}

\author{
Dissertation \\ zur Erlangung des wirtschaftswissenschaftlichen Doktorgrades \\ des Fachbereichs Wirtschaftswissenschaften der \\ Georg-August-Universität Göttingen
}

vorgelegt von

Dipl.-Ing. Maximilian Eichhorn

von Münster / Westfalen

Göttingen 2000 
Erstgutachter

Zweitgutachter

Tag der mündlichen Prüfung
Prof. Dr. Dr.h.c. Jürgen Bloech

Prof. Dr. Helmut Brede

15. Dezember 2000 
Für Susanne 
Wenn ein Unternehmer eine Fehlentscheidung getroffen hat, so wird er diesen schmerzlichen Sachverhalt aus seiner Gewinn- und Verlustrechnung erfahren. Er wird versuchen, sich an die Regel zu halten, dass man einen Fehler nur einmal machen darf. So wird er seine Erfahrungen sammeln und aus den Erfahrungen lernen. In diesem Sinne schafft das Planspiel eine höchstmögliche Annäherung an das praktische Sammeln von Erfahrungen. Es ist eine aktive Form des Lernens, ein Lernen durch Handeln, durch Sammeln von Erfahrungen aus eigenen Entscheidungen. Dabei kann oftmals aus falschen Entscheidungen im Planspiel mehr gelernt werden als aus richtigen.

Ludwig Pack

Pack (Planspiele, 1968) Seite 13. Ludwig Pack war Professor am Institut für industrielle Unternehmensforschung der Universität Münster. 


\section{Vorwort}

Angesichts der zunehmenden Arbeitsteilung, Verkettung von Wertschöpfungsbeiträgen, Komponentenfertigung, Roboterisierung, Produktionsverfahrensanpassung, zwischenbetrieblichen und internationalen Warenströme bis hin zur Globalisierung gewinnt die Logistik immer größere Bedeutung. Einem Teilaspekt widmet sich die vorliegende Arbeit. Sie untersucht das betriebliche Verhalten logistischer Systeme im Zeitablauf. Um die ständig ablaufenden Logistikvorgänge in sich entwickelnden Entscheidungssituationen abzubilden, wird das Mittel der Simulation eingesetzt. Dabei kommt es darauf an, die Wirklichkeit stringent einzufangen und die extern und intern determinierten wechselnden Bedingungen $\mathrm{zu}$ erfassen und innerhalb ihrer Grenzen zielorientierte Entscheidungen vorzubereiten.

Vorgestellt wird eine Industrieunternehmung, die zwei Produkte auf bis zu drei verschiedenen Märkten absetzen will. Am Planspiel - eine besondere Art der Simulation

- nehmen fünf virtuelle Unternehmen bzw. Spielgruppen teil. Bei konsequenter Konstruktion des Modells erlaubt diese Methode, betriebliche Entscheidungen in einem komplexen ökonomischen Umfeld auf Grundlage aufeinander folgender operativer Maßnahmen und Ergebnisse zu treffen.

Das hier simulierte Unternehmen ist zwar unabhängig von Strukturen oder Zahlen einer speziellen Firma, es wird jedoch eine mehrstufige Verbundproduktion dargestellt, wie sie z.B. in der Automobilindustrie oder bei anderen Serienfertigungen vorkommt. Zahlen, Parameter und sonstige Informationen sind frei gewählt.

Der vorliegende Text ist nur ein Teil der Arbeit. Er skizziert die Grundlagen der Logistiktheorie, gibt einen Einblick in Art und Funktionsweise von Planspielen und beschreibt detailliert die Modellbildung des simulierten Unternehmens. Auf diesem Konzept sind zwei vollständige Computerprogramme entwickelt und programmiert worden: Ein Unternehmensmodell, das für die Entscheidungsfindung und für die Entscheidungsauswertung benötigt wird, sowie ein Marktmodell, das den ökonomischen Rahmen in Form von Absatzmöglichkeiten oder (Markt-) Parametern bildet. 
Die vorliegende Arbeit wurde im Wintersemester 2000 / 2001 von der Fakultät für Wirtschaftswissenschaften der Universität Göttingen als Dissertation angenommen. Mein Dank gilt besonders meinem Doktorvater, Herrn Prof. Dr. Dr.h.c. Jürgen Bloech, Vorstand des Instituts für Betriebswirtschaftliche Produktions- und Investitionsforschung. Zahlreiche Gespräche und Diskussionen mit Prof. Bloech und seinem Team unterstützten mich auf meinem Weg zu LogisticPLUS.

Ebenso danke ich Herrn Prof. Dr. Helmut Brede, Vorstand des Instituts für Rechnungsund Prüfungswesen privater und öffentlicher Betriebe, für zahlreiche wertvolle Hinweise, Herrn Prof. Dr. Günter Gabisch, Ordinarius im Seminar für Volkswirtschaftslehre, für die Übernahme des volkswirtschaftlichen Prüfungsteiles sowie dem ehemaligen Alleinvorstand der FRIATEC Aktiengesellschaft, Herrn Senator h.c. Prof. Dr. Friedrich Reutner, für die Möglichkeit, eine berufsbegleitende Dissertation anzufertigen. Den Herren Dipl.-Volkswirt Herbert Rüscher, Institut für Betriebswirtschaftliche Produktions- und Investitionsforschung der Universität Göttingen, und cand. inf. techn. Andreas Roessle, Universität Mannheim, bin ich für ihre Unterstützung bei informationstechnischen Fragen ebenfalls zu Dank verpflichtet.

Der größte Dank gebührt meinen Eltern. Sie haben mir und meinen beiden Geschwistern ein glückliches und geborgenes familiäres Umfeld gegeben, meine persönliche Entwicklung überdurchschnittlich gefördert und mich in meinen Entscheidungen immer unterstützt. 


\section{Inhaltsverzeichnis}

\begin{tabular}{|c|c|}
\hline torwort & \\
\hline Inhaltsverzeichnis & IIII \\
\hline Abkürzungsverzeichnis & VII \\
\hline Abbildungsverzeichnis & VIII \\
\hline Tabellenverzeichnis & $\mathrm{X}$ \\
\hline 1 Problemstellung und Vorgehensweise & 1 \\
\hline 2 Logistik in Theorie und Praxis & 7 \\
\hline 2.1 Grundlagen der industriellen Logistik & 7 \\
\hline 2.2 Logistische Funktionen und ihre Aufgaben & 15 \\
\hline $2.2 .1 \quad$ Beschaffungslogistik & 16 \\
\hline 2.2.2 Produktionslogistik & 17 \\
\hline $2.2 .3 \quad$ Distributionslogistik & 19 \\
\hline 2.2.4 Entsorgungslogistik & 21 \\
\hline 2.3 Systeme der industriellen Logistik & 22 \\
\hline 2.3.1 Auftragsabwicklung als übergreifender Prozess & 22 \\
\hline 2.3.2 Die Lagerhaltung als Grundlage des Geschäftes & 23 \\
\hline 2.3.3 Das Lagerhaus als logistisches Zentrum & 26 \\
\hline 2.3.4 Die Verpackung in Logistiksystemen & 28 \\
\hline 2.3.5 Transporte als Hauptfunktionen der Logistik & 31 \\
\hline 2.4 Kenngrößen der Logistik & 34 \\
\hline 2.5 Aktuelle Entwicklungen und Probleme & 36 \\
\hline 2.6 Einschlägige Berufe und Aufgaben & 40 \\
\hline 3 Planspiele und Unternehmenssimulationen für Konkurrenzsituationen & 45 \\
\hline 3.1 Merkmale von Planspielen & 45 \\
\hline 3.1.1 Historische Entwicklung & 45 \\
\hline 3.1.2 Anwendungsgebiete von Planspielen & 46 \\
\hline 3.1.3 Vor- und Nachteile von Planspielen & 48 \\
\hline 3.2 Planspiele in der betriebswirtschaftlichen Aus- und Weiterbildung & 50 \\
\hline 3.2.1 $\quad$ Aufbau und Funktionsweise & 50 \\
\hline 3.2.2 Arten und Einteilung von Planspielen & 53 \\
\hline 3.2.3 $\quad$ Generelle Planspiele & 54 \\
\hline 3.2.3.1 EpUS - Ein-Platz-Unternehmens-Simulation & 55 \\
\hline
\end{tabular}


3.2.3.2 BO-Cash

\begin{tabular}{|lrr}
\hline 3.2 .4 & Funktionsorientierte Planspiele & 57 \\
\hline
\end{tabular}

\begin{tabular}{|lll}
\hline 3.2 .4 .1 & PUMA - Produktions- und Marketing-Simulation & 58 \\
\hline
\end{tabular}

\begin{tabular}{llr}
\hline 3.2 .4 .2 & SIM-LOG - Simulation zur Logistik & 59 \\
\hline
\end{tabular}

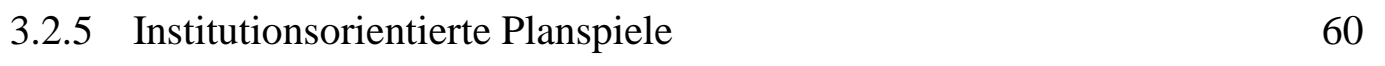

\begin{tabular}{lll}
\hline 3.2 .5 .1 & PENTA - Planspiel Entsorgungs- und Abfallwirtschaft & 60
\end{tabular}

3.2.5.2 KLIMA $^{\text {FORTE }}$ - Klinikmanagement mit FallpauschalenFinanzierung 61

\begin{tabular}{|lll}
\hline 3.2 .6 & Anwendungsformen von betriebswirtschaftlichen Planspielen & 63 \\
\hline
\end{tabular}

\begin{tabular}{|lll}
4 & Konzeption des Planspiels LogisticPLUS & 65 \\
\hline
\end{tabular}

\begin{tabular}{lr}
\hline 4.1 Grundlagen & 65 \\
\hline
\end{tabular}

\begin{tabular}{|lll}
\hline 4.1 .1 & Zielsetzung von LogisticPLUS & 65 \\
\hline
\end{tabular}

4.1 .2 Das Unternehmen 66

\begin{tabular}{|lll}
\hline 4.1 .3 & Marktstellung und Wettbewerber & 68 \\
\hline
\end{tabular}

\begin{tabular}{lll}
4.1 .4 & Entscheidungen bei LogisticPLUS & 70 \\
\hline
\end{tabular}

4.1 .5 Marktmodell und ausführbare Computerprogramme 71

4.1 .6 Das Computerprogramm für den Spieler 7

\begin{tabular}{|ll}
\hline 4.2 Leistungsströme bei LogisticPLUS & 75 \\
\hline
\end{tabular}

\begin{tabular}{|lll}
4.3 Verschiedene Entscheidungsbereiche & 80 \\
\hline
\end{tabular}

\begin{tabular}{|llr}
\hline $4.3 .1 \quad$ Absatz und Marketing & 80 \\
\hline
\end{tabular}

\begin{tabular}{|llr}
\hline 4.3 .1 .1 & Marktforschung und Wettbewerbsberichte & 80 \\
\hline
\end{tabular}

\begin{tabular}{|llr}
\hline 4.3 .1 .2 & Preispolitik und ihre Wirkung & 82 \\
\hline 4.3 .1 .3 & Absatzuntersützung & 83
\end{tabular}

\begin{tabular}{|lll}
\hline 4.3 .1 .3 Absatzunterstützung & 83 \\
\hline
\end{tabular}

\begin{tabular}{|llr}
\hline 4.3 .2 & Das Produktionssystem im Planspiel & 87 \\
\hline
\end{tabular}

\begin{tabular}{|lll|}
\hline 4.3 .2 .1 & Fertigung und Fertigungssteuerung & 88 \\
\hline
\end{tabular}

\begin{tabular}{|lll}
\hline 4.3 .2 .2 & Die Montage der Verkaufsartikel & 89 \\
\hline
\end{tabular}

\begin{tabular}{|lll}
\hline 4.3 .2 .3 & Recycling ausgesonderter Verkaufsartikel & 91 \\
\hline
\end{tabular}

\begin{tabular}{|lll}
\hline 4.3 .3 & Die Logistik und ihre Komponenten & 92 \\
\hline
\end{tabular}

\begin{tabular}{llr}
\hline 4.3 .3 .1 & Beschaffungslogistik & 92 \\
\hline 4.3 .3 .2 & Produktionslogiktik & 95
\end{tabular}

\begin{tabular}{|llr}
\hline 4.3 .3 .2 & Produktionslogistik & 95 \\
\hline 4.3 .3 .3 & Diktributionslogistik & 96
\end{tabular}

\begin{tabular}{|lll}
\hline 4.3 .3 .3 & Distributionslogistik & 96 \\
\hline 4.3 .3 .4 & Entsorgungslogik & 97
\end{tabular}

\begin{tabular}{lll}
\hline 4.3 .3 .4 & Entsorgungslogistik & 97 \\
\hline
\end{tabular}

\begin{tabular}{llr}
4.3 .4 & Qualitätssicherung & 98 \\
\hline
\end{tabular}

\begin{tabular}{lll}
4.3 .5 & Finanzierung & 100 \\
\hline
\end{tabular}

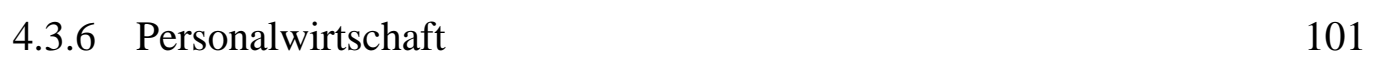

\begin{tabular}{lll}
\hline 4.3 .6 .1 & Fach- und Hilfsarbeiter & 101 \\
\hline
\end{tabular}

\begin{tabular}{llc}
\hline 4.3 .6 .2 & Sozialleistungen & 103 \\
& &
\end{tabular} 
4.3.6.3 Verwaltung und Vorstand 104

4.3 .7 Eingabemasken für verschiedene Bereiche 106

4.4 Interne und externe Rechnungsinformationen 114

\begin{tabular}{lll}
\hline 4.4.1 & Kostenrechnung & 114 \\
\hline
\end{tabular}

\begin{tabular}{lll}
\hline 4.4.1.1 & Bewertungsspielräume & 115 \\
\hline
\end{tabular}

\begin{tabular}{|llr|}
\hline 4.4 .1 .2 & Kalkulation der Halbfabrikate (Herstellungskosten A) & 117 \\
\hline \hline
\end{tabular}

\begin{tabular}{lll}
\hline 4.4.1.3 Kalkulation der Fertigfabrikate (Herstellungskosten B) & 118 \\
\hline 4.4 .17
\end{tabular}

\begin{tabular}{|llr}
\hline 4.4 .1 .4 & Kalkulation der Verkaufsartikel (Herstellungskosten C) & 120 \\
\hline \hline .
\end{tabular}

\begin{tabular}{|lll}
\hline 4.4.1.5 Kalkulation der Verkaufsartikel (Selbstkosten) & 123 \\
\hline
\end{tabular}

\begin{tabular}{lll}
\hline .4 .2 & Gewinn- und Verlustrechnung & 126 \\
\hline
\end{tabular}

\begin{tabular}{lll}
4.4 .3 Bilanz & 130 \\
\hline
\end{tabular}

4.4 .4 Liquiditätsrechnung 136

\begin{tabular}{lll}
\hline 4.4.5 & Finanzkennzahlen & 139 \\
\hline
\end{tabular}

4.5 Einschlägige Betriebskennzahlen 141

\begin{tabular}{lll}
4.5 .1 & Vertrieb & 142 \\
\hline
\end{tabular}

\begin{tabular}{|lll}
\hline 4.5 .2 & Produktion, Qualität und Reklamationen & 145 \\
\hline
\end{tabular}

\begin{tabular}{lll}
4.5 .3 Anlagevermögen & 146 \\
\hline 4.5 .4 & Unlaufvermögen
\end{tabular}

4.5 .4 Umlaufvermögen 148

\begin{tabular}{lll}
\hline .5 .5 & Transporte & 151 \\
\hline .5 .6 & Persan &
\end{tabular}

4.5 .6 Personal, Verwaltung und Finanzierung 152

4.6 Marktinformationen 156

\begin{tabular}{lll}
$4.6 .1 \quad$ Allgemeines & 156 \\
\hline
\end{tabular}

4.6 .2 Informationen über Marktpreise 156

4.6 .3 Informationen über Parameter 161

4.6.4 Ergebnisse der Marktforschung 165

4.7 Reihenfolge der Simulation 172

\begin{tabular}{|ll}
5 Konzeption des Spielleitermoduls & 175 \\
\hline
\end{tabular}

\begin{tabular}{ll}
\hline 1 Aufbau und Bedienung & 175 \\
\hline
\end{tabular}

\begin{tabular}{ll}
\hline .2 Steuerungsgrößen & 177 \\
\hline
\end{tabular}

\begin{tabular}{ll}
\hline .3 Auswertungen & 180 \\
\hline
\end{tabular}

5.4 Das Computerprogramm für den Spielleiter 182

6 Logistikausbildung mit LogisticPLUS 185

6.1 Zielgruppenidentifikation 185

6.2 Einsatzmöglichkeiten 186

6.3 Seminarkonzeption und Lehrmodule 190 
\begin{tabular}{lll}
6.3 .1 & Seminareinstieg & 190 \\
\hline 6.3 .2 & Ser
\end{tabular}

6.3.2 Seminarablauf 193

6.3 .3 Nachbereitung des Seminars 196

\begin{tabular}{lll}
7 Zusammenfassung & 197 \\
\hline
\end{tabular}

\begin{tabular}{|lr}
\hline Auszug aus dem Programmcode & 201 \\
\hline
\end{tabular}

Beispiel 1: Überprüfung der Eingabe auf eine korrekten Wert 201

Beispiel 2: Berechnung der maximal möglichen Produktion 202

Literaturverzeichnis 205

Versicherung an Eides Statt 214

\begin{tabular}{|lr}
\hline Autor 215 \\
\hline
\end{tabular} 


\section{Abkürzungsverzeichnis}

\begin{tabular}{|c|c|c|c|}
\hline Abb. & Abbildung & $\mathrm{kg}$ & Kilogramm \\
\hline bzw. & beziehungsweise & LKW & Lastkraftwagen \\
\hline ca. & circa & $\mathrm{m}$ & Meter \\
\hline \multirow[t]{2}{*}{ Cap } & Capacity (Kapazität, 1 Cap & $\mathrm{m}^{3}$ & Kubikmeter \\
\hline & entspricht 1 Stunde) & $\min$ & Minute \\
\hline $\mathrm{CD}$ & Compact Disc & Mio. & Millionen \\
\hline $\mathrm{cm}^{3}$ & Kubikzentimeter & Mrd. & Milliarden \\
\hline d.h. & das heißt & PPS & Produktionsplanung- und \\
\hline $\mathrm{dm}^{3}$ & Kubikdezimeter & & Steuerung \\
\hline engl. & englisch & QM & Qualitätsmanagement \\
\hline et al. & et aliter (und andere) & ROI & Return on Investment \\
\hline etc. & et cetera & & (Rentabilität des investierten \\
\hline $\mathrm{F} \& \mathrm{E}$ & Forschung und Entwicklung & & Kapitals) \\
\hline ff. & fortlaufende (Seiten) & St. & Stïck \\
\hline \multirow[t]{2}{*}{ FAZ } & Frankfurter Allgemeine & Tab. & Tabelle \\
\hline & Zeitung & u.a. & unter anderem \\
\hline FK-Zinsen & Fremdkapitalzinsen & vgl. & vergleiche \\
\hline GabSt & Gabelstapler & VK-Art & 1 Verkaufsartikel \\
\hline \multirow[t]{2}{*}{$\mathrm{GuV}$} & Gewinn- und & WWW & World Wide Web (Teil des \\
\hline & Verlustrechnung & & Internet) \\
\hline \multirow[t]{3}{*}{ HTML } & Hypertext Markup & z.B. & zum Beispiel \\
\hline & Language (Sprache, um & & \\
\hline & Informationen im WWW & & \\
\hline
\end{tabular}

Hrsg. Herausgeber 


\section{Abbildungsverzeichnis}

\begin{tabular}{|c|c|c|}
\hline A bb. 1: & Funktionen der Logistik und Organisationsformen der Logistik & 10 \\
\hline Abb. 2: & Logistische Funktionen & 16 \\
\hline \multirow[t]{2}{*}{ Abb. 3: } & Komponenten des Lagerbestandes bei Vorratsergänzung und & \\
\hline & Vorratssicherung & 25 \\
\hline Abb. 4: & Lagertypenstruktur & 27 \\
\hline Abb. 5: & Einflussgrößen auf Verpackungsentscheidungen & 31 \\
\hline Abb. 6: & Grundstruktur von Planspielen & 51 \\
\hline Abb. 7: & Reaktionskette in Unternehmensspielen & 52 \\
\hline Abb. 8: & Struktur (Beispiel) des LogisticPLUS - Unternehmens & 68 \\
\hline Abb. 9: & Marktsituation bei LogisticPLUS & 69 \\
\hline Abb. 10: & Struktur der Planspieloberfläche & 72 \\
\hline Abb. 11: & Abwicklung einer Periode & 74 \\
\hline Abb. 12: & Leistungs- und Finanzbereich bei LogisticPLUS & 76 \\
\hline Abb. 13: & Leistungsfluss LogisticPLUS & 77 \\
\hline Abb. 14: & Absatzstruktur LogisticPLUS & 79 \\
\hline Abb. 15: & Bestimmung des Marktvolumens & 82 \\
\hline Abb. 16: & Preisabweichung vom Durchschnittspreis & 83 \\
\hline Abb. 17: & Wirksamkeit der Werbung (effektive Werbung) & 85 \\
\hline Abb. 18: & Werbefaktor & 86 \\
\hline Abb. 19: & Berechnung und Begrenzung des Preisfaktors & 87 \\
\hline Abb. 20: & Stücklisten für die VK-Artikel Singulus und Combilus & 89 \\
\hline Abb. 21: & Verkaufsartikel Singulus & 90 \\
\hline Abb. 22: & Verkaufsartikel Combilus & 91 \\
\hline Abb. 23: & Wirksamkeit der Qualitätsfaktoren & 99 \\
\hline Abb. 24: & Einfluss der Sozialabgaben auf den Krankenstand & 104 \\
\hline Abb. 25: & Eingabemaske Absatz und Marketing & 106 \\
\hline Abb. 26: & Eingabemaske Einkauf und Produktion & 108 \\
\hline Abb. 27: & Eingabemaske Maschinen & 109 \\
\hline Abb. 28: & Eingabemaske Silos & 110 \\
\hline Abb. 29: & Eingabemaske Lager & 111 \\
\hline Abb. 30: & Eingabemaske Transporte & 112 \\
\hline Abb. 31: & Eingabenmaske Diverse & 113 \\
\hline
\end{tabular}


Abb. 32: Ausgabemaske Herstellungskosten A 118

\begin{tabular}{llr}
\hline Abb. 33: & Ausgabemaske Herstellungskosten B & 120 \\
\hline
\end{tabular}

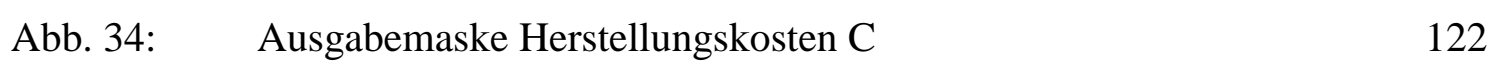

\begin{tabular}{|ll|}
\hline Abb. 35: Ausgabemaske Selbstkosten & 124 \\
\hline
\end{tabular}

Abb. 36: Kennzahlen Produktkalkulation (Herstellungskosten) 125

\begin{tabular}{lll}
\hline Abb. 37: Ausgabemaske GuV & 128 \\
\hline
\end{tabular}

\begin{tabular}{lll}
\hline Abb. 38: & Ausgewählte Kennzahlen GuV & 129 \\
\hline
\end{tabular}

\begin{tabular}{|lr}
\hline Abb. 39: Ausgabemaske Bilanz & 132 \\
\hline
\end{tabular}

\begin{tabular}{|lr|}
\hline Abb. 40: $\quad$ Ausgewählte Bilanzkennzahlen & 133 \\
\hline
\end{tabular}

\begin{tabular}{|lr|}
\hline Abb. 41: Kennzahlen Anlagevermögen & 134 \\
\hline
\end{tabular}

\begin{tabular}{|ll|}
\hline Abb. 42: Kennzahlen Umlaufvermögen & 135 \\
\hline
\end{tabular}

\begin{tabular}{lll}
\hline Abb. 43: Ausgabemaske Liquidität & 137 \\
\hline
\end{tabular}

\begin{tabular}{lll}
\hline Abb. 44: Liquidität & 138 \\
\hline
\end{tabular}

\begin{tabular}{lll}
\hline Abb. 45: $\quad$ Ausgabemaske Cashflow und Eigenkapitalrentabilität & 141 \\
\hline
\end{tabular}

\begin{tabular}{lll}
\hline Abb. 46: Ausgabemaske Kennzahlen Vertrieb & 143 \\
\hline
\end{tabular}

\begin{tabular}{lll}
\hline Abb. 47: Ausgewählte Vertriebskennzahlen & 144 \\
\hline
\end{tabular}

Abb. 48: Ausgabemaske Kennzahlen Produktion, Qualität und Reklamationen 146

Abb. 49: Ausgabemaske Kennzahlen Anlagevermögen 147

\begin{tabular}{lll}
\hline Abb. 50: Ausgabemaske Kennzahlen Umlaufvermögen & 149 \\
\hline
\end{tabular}

Abb. 51: Graphische Darstellung Auslastung der Lagerstellen 150

\begin{tabular}{|lr|}
\hline Abb. 52: & Ausgabemaske Kennzahlen Transporte \\
\hline
\end{tabular}

Abb. 53: Graphische Darstellung Personalstand 153

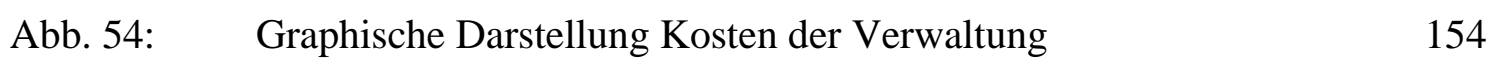

Abb. 55: Ausgabemaske Kennzahlen Personal, Verwaltung und Finanzierung 155

\begin{tabular}{lll}
\hline Abb. 56: Ausgabemaske Marktpreise Einkauf & 157 \\
\hline
\end{tabular}

Abb. 57: Ausgabemaske Marktpreise Silos, Lager und Maschinen 158

\begin{tabular}{lll}
\hline Abb. 58: Ausgabemaske Marktpreise Transport & 159 \\
\hline
\end{tabular}

Abb. 59: Ausgabemaske Marktpreise Personal und Verwaltung 160

\begin{tabular}{|lll}
\hline Abb. 60: & Ausgabemaske Marktpreise Finanzierung und Sonstiges & 161 \\
\hline
\end{tabular}

\begin{tabular}{|ll|}
\hline Abb. 61: Ausgabemaske Parameter Stücklisten & 162 \\
\hline
\end{tabular}

Abb. 62: Ausgabemaske Parameter Maschinen 163

\begin{tabular}{lll}
\hline Abb. 63: & Ausgabemaske Parameter Transporte & 164 \\
\hline
\end{tabular}

Abb. 64: Ausgabemaske Parameter Abschreibungen, Personal und Kredite 165

\begin{tabular}{lll}
\hline Abb. 65: & Ausgabemaske Marktforschung klein (Teil 1) & 166 \\
\hline
\end{tabular} 


\begin{tabular}{llr|}
\hline Abb. 66: & Ausgabemaske Marktforschung klein (Teil 2) & 167 \\
\hline Abb. 67: & Auszug Graphische Darstellung Kennzahlen Wettbewerb (Teil 1) & 168 \\
\hline Abb. 68: & Ausgabemaske Marktforschung groß (Teil 1) & 169 \\
\hline Abb. 69: & Ausgabemaske Marktforschung groß (Teil 2) & 170 \\
\hline Abb. 70: & Auszug Graphische Auswertung Kennzahlen Wettbewerb (Teil 2) & 171 \\
\hline Abb. 71: & Reihenfolge der Simulation & 172 \\
\hline Abb. 72: & Abwicklung der Simulation & 176 \\
\hline Abb. 73: & Nachfragevorgabe durch die Spielleitung & 178 \\
\hline Abb. 74: & Einstellungsmöglichkeiten Nachfrage (Spielleitung) & 180 \\
\hline Abb. 75: & Spielleiterauswertung Umsatz & 181 \\
\hline Abb. 76: & Spielleiterauswertung Effektive Faktoren & 182 \\
\hline
\end{tabular}

\section{Tabellenverzeichnis}

\begin{tabular}{|c|c|c|}
\hline Tab. 1: & Zielsystem der Logistik & 12 \\
\hline Tab. 2: & Einfluss des Lagerbestandes auf den ROI & 13 \\
\hline Tab. 3: & Unterscheidung von Lagerhausarten nach ihrer Funktion & 26 \\
\hline Tab. 4: & Grundfunktionen der Verpackung & 29 \\
\hline Tab. 5: & Kosten-, Angebots- und Bedarfsmatrix eines Transportproblems & 33 \\
\hline Tab. 6: & Erste Lösung des Transportproblems & 33 \\
\hline Tab. 7: & Optimale Lösung des Transportproblems & 34 \\
\hline Tab. 8: & Produktmerkmale bei BO-Cash & 56 \\
\hline Tab. 9: & Entscheidungen bei BO-Cash & 57 \\
\hline Tab. 10: & Entscheidungen bei PUMA & 58 \\
\hline Tab. 11: & Entscheidungen bei SIM-LOG & 60 \\
\hline Tab. 12: & Entscheidungen bei PENTA & 61 \\
\hline Tab. 13: & Entscheidungen LogisticPLUS & 70 \\
\hline Tab. 14: & Berechnung der Werbefaktoren & 86 \\
\hline Tab. 15: & Rohstoffeinsatz und Volumen der Produkte 1 und 2 & 88 \\
\hline Tab. 16: & Produktionsdauer Fertigung Produkte 1 und 2 & 88 \\
\hline Tab. 17: & Gesamtproduktivität je Maschine und Monat & 89 \\
\hline Tab. 18: & Stückliste für den VK-Artikel Combilus & 90 \\
\hline Tab. 19: & Einkaufswert und Anfahrtsgebühren für Rohstoffe und Zukaufpr & 294 \\
\hline
\end{tabular}




\begin{tabular}{|c|c|c|}
\hline Tab. 20: & Beispiel Volumens- und Stückzahlreduktion bei der Einlagerung & 94 \\
\hline Tab. 21: & Benötigte Gabelstapler-Kapazitäten für innerbetriebliche Transporte & 95 \\
\hline Tab. 22: & Kosten für Absatztransporte & 97 \\
\hline Tab. 23: & Einfluss verschiedener Aufwendungen & 99 \\
\hline Tab. 24: & Berechnung der Unterstützungsfaktoren in der Planungsphase & 100 \\
\hline Tab. 25: & Zinssätze für Finanzierungen Kreditzinsen & 101 \\
\hline Tab. 26: & Lohn-, Einstellungs- und Entlassungskosten & 102 \\
\hline Tab. 27: & Kapazitäten pro Fach- und Hilfsarbeiter & 102 \\
\hline Tab. 28: & Benötigte Personalkapazitäten & 102 \\
\hline Tab. 29: & Umsatzabhängige Kosten der Verwaltung & 105 \\
\hline Tab. 30: & Personalabhängige Kosten der Verwaltung & 105 \\
\hline Tab. 31: & Ermittlung der Herstellungskosten in Handels- und Steuerbilanz & 115 \\
\hline Tab. 32: & Kalkulation der Halbfabrikate (Herstellungskosten A) & 117 \\
\hline Tab. 33: & Kalkulation der Fertigfabrikate (Herstellungskosten B) & 119 \\
\hline Tab. 34: & Kalkulation der Verkaufsartikel (Herstellungskosten C) & 121 \\
\hline Tab. 35: & Kalkulation der Verkaufsartikel (Selbstkosten) & 123 \\
\hline Tab. 36: & Gliederung der GuV & 126 \\
\hline Tab. 37: & Gliederung der Bilanz & 130 \\
\hline Tab. 38: & Liquiditätsrechnung & 136 \\
\hline Tab. 39: & Allgemeine Berechnung des Cashflow & 139 \\
\hline Tab. 40: & Berechnung des Cashflow bei LogisticPLUS & 140 \\
\hline Tab. 41: & Beispiel Eigenkapitalrentabilität & 140 \\
\hline Tab. 42: & Innerbetriebliche Transporte & 151 \\
\hline Tab. 43: & Auswahl Steuerungsgrößen des Spielleiters & 179 \\
\hline Tab. 44: & Lehrmodule und Inhalte & 192 \\
\hline
\end{tabular}




\title{
1 Problemstellung und Vorgehensweise
}

\author{
Wir lernen am meisten aus Erfahrungen, \\ aber wir erfahren häufig nicht, wie sich \\ unsere wichtigsten Entscheidungen aus- \\ wirken.
}

Peter M. Senge

Die zunehmende Verschärfung des Wettbewerbs, wachsende Transparenz und Komplexität wirtschaftlicher Abläufe sowie die immer schneller werdende Wandlung der Unternehmensumwelt fordern professionelles, qualifiziertes und weitblickendes Management, um Unternehmen erfolgreich zu führen.

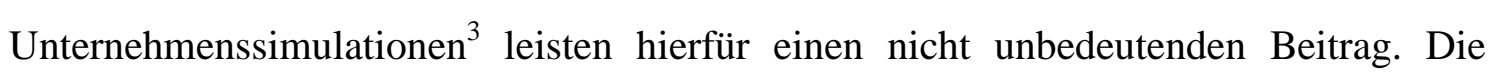
vorliegende Arbeit bildet die Basis für ein sogenanntes Planspiel ${ }^{\natural}$ aus dem Bereich der Unternehmensführung. Im Vordergrund stehen die logistischen Aspekte von Beschaffung, Produktion und Distribution. Die Bezeichnung „Planspiel“ charakterisiert das Vorhaben allerdings nur unvollkommen. Es geht um eine besondere Art der Modellbildung für im Wettbewerb stehende Unternehmen. Angestrebt wird die Konstruktion eines virtuellen Systems von Märkten und Unternehmungen, die im Zeitablauf durch eigene Entscheidungen und die Veränderung von Rahmenbedingungen unterschiedliche Entwicklungen durchlaufen. Ein derartiges Modell von Märkten und Unternehmungen kann an die Unternehmensführung ähnliche Ansprüche stellen, wie sie in realen Unternehmungen auftauchen. Anders als in schriftlichen Darstellungen und mündlichen Vorträgen können Teilnehmer in simulierten Unternehmungen die Bedeutung der logistischen Systeme besser verstehen. Diese Erkenntnisse bieten eine gute Informationsbasis für richtige Entscheidungen. Dieses sogenannte Lernhandeln ist

2 Senge (Disziplin, 1999) Seite 35. Peter M. Senge ist Leiter des ,Systems Thinking and Organizational Learning Program' an der Sloan School of Management des Massachusetts Institute of Technology.

3 Simulation, von Lateinischer Herkunft, bedeutet in Wissenschaft und Technik die modellhafte Nachbildung eines beliebigen Systems oder Prozesses durch ein anderes (kybernetisches) System bzw. einen anderen Prozess und das Experimentieren mit diesem Modell. Vgl. Brockhaus (LexiROM, 1995).

4 Weitere Bezeichnungen für Planspiele sind Unternehmensplanspiel, Unternehmensspiel, Unternehmenssimulation, Management Game, Business Game oder Top Management Decision Simulation. 
als Funktion primär auf die Ausbildung der subjektiven Handlungsfähigkeit bzw. den Auf- und Ausbau kognitiver Strukturen gerichtet. ${ }^{[}$

Die genannten logistischen Funktionen in der Beschaffung, Produktion und Distribution sind ein wesentliches Element der Unternehmensstrategie ${ }^{6}$ und insoweit ein entscheidender Wettbewerbsfaktor geworden. ${ }^{7}$ Darauf weisen zahlreiche wissenschaftliche Abhandlungen, die Angebote von logistischen Dienstleistungen und die zunehmende Nachfrage nach Logistikmanagern hin. Aufgaben der Logistik erwachsen auch aus der Globalisierung. Just-in-time, Modular Sourcing und Global Sourcing sind dafür einschlägige Stichworte. Die ursprünglich auf eine Betriebsstätte und einzelne Bauteile reduzierten Beschaffungs- und Steuerungsaufgaben sind in den letzten Jahren deutlich erweitert worden: So werden z.B. nicht nur einzelne Bauteile, sondern ganze Baugruppen international ausgeschrieben. Diese müssen vom potenziellen Lieferanten zu einem bestimmten Zeitpunkt direkt in ein Produktionswerk geliefert werden. Zugleich findet eine Regionalisierung von Produktionsnetzwerken statt. $^{\mathrm{B}}$ Dies bedeutet, dass die Produktion bzw. Wertschöpfung nicht mehr durchgängig an einem Ort geschieht, sondern dass Firmen oder Firmenverbünde bestimmte Produkte oder Baugruppen an unterschiedlichen Orten fertigen lassen und diese z.B. im Land des späteren Kunden montieren lassen. Zudem haben die sich wandelnden Märkte durch kürzere Produktlebenszyklen, gestiegene Qualitätsansprüche und zunehmende Variantenvielfalt direkten Einfluss auf die gesamten Wertschöpfungskette und damit auf die Logistik. Wie im Rahmen dieser komplexen Entwicklungen richtige Entscheidungen zu treffen sind, dieser Aufgabe widmet sich diese Arbeit.

Planspiele sind für Entscheidungsfindungen unter den oben genannten Aspekten sehr hilfreich. Sie haben sowohl in der Analyse der unternehmerischen Problemstellungen als

\footnotetext{
Vgl. Achtenhagen et al. (Lernhandeln, 1992) Seite 83.

6 Die Logistik kann im Rahmen der strategischen Planung der Funktionsbereiche im Gegensatz zu F\&E, Produktion oder Absatz als übergreifende Funktion betrachtet werden. Vgl. Götze / Mikus (Strategisches Management, 1999) Seite 185.

7 Vgl. Merkel (Managementsysteme, 1995) Seite 15 oder: Porter (Wettbewerbsvorteile, 1992) Seite 650: Veränderungen in der Logistik des Warenzugangs und im Kundendienst: Ein Herausforderer kann einen Branchenführer angreifen, indem er Dinge wie Produktunterstützung, Kundendienst, Auftragsabwicklung oder physische Distribution verändert. So hat beispielsweise die Neuausrichtung der Wertaktivitäten bei Federal Express, einem der weltweit größten Logistikunternehmen, die relative Kostenposition erheblich verbessert.

8 Kuhn (Unternehmensplanung, 1998) ohne Seitenangabe.
} 
auch in der Aus- und Weiterbildung einen hohen Stellenwert. ${ }^{2}$ Sie vermitteln ein Gespür für die sich stellenden Aufgaben, zeigen Engpässe oder kritische Entscheidungsvariable auf und lassen den Benutzer Schwierigkeiten erkennen, nachvollziehen und beheben.

Die zentralen Fragestellungen der vorliegenden Arbeit lauten:

1. Was müssen Logistiker verstehen, erlernen und entscheiden können ?

2. Bietet das Instrument „Planspiel“ eine geeignete Antwort auf diese Frage ?

3. Was leistet das vorliegende Planspiel dazu ?

Es wird sich zeigen, dass mit Hilfe des entwickelten Planspiels der (generelle) Logistiker die Markt- und Absatzeinflüsse begreifen und für seine originäre Arbeit berücksichtigen lernt. Er wird die Zusammenhänge zwischen Marketing, Absatz und Logistik und die daraus folgenden wirtschaftlichen Auswirkungen anhand der betriebsund marktwirtschaftlichen Auswertungen schneller erkennen können und damit für seine tägliche Arbeit eine Verbesserung der logistischen Entscheidungen erreichen.

Den Grundlagen logistischer Fragestellungen in einer Unternehmung dient das zweite Kapitel. Logistik wird dabei als Summe von unterschiedlichen Funktionen verstanden. Unterschieden werden Einkauf, Beschaffung, Transport, Lagerhaltung, PPS, Distribution, Fabrikplanung und Entsorgung. 10 Der Schwerpunkt der Beschreibungen liegt dabei auf jenen Funktionen, die sich für die spätere Modellierung bzw. für die ausführbaren Computerprogramme als wichtig erweisen. Im logistischen Bereich des Planspiels sind dies vor allem Entscheidungen über Beschaffungsmengen, Lagerhaltung sowie innerbetriebliche und außerbetriebliche Transportressourcen. Im Weiteren werden die für das Logistiksystem wesentlichen verrichtungsspezifischen Subsysteme Auftragsabwicklung, Lagerhaltung, Lagerhaus, Verpackung und Transport erörtert. Aktuelle Entwicklungen und Problemfelder sowie kurze Berufsbeschreibungen im logistischen Bereich schließen dieses Kapitel ab.

9 Vgl. Drössler (Planspiele, 1998): Virtueller Bankrott: Mit Planspielen sollen Manager lernen ein Unternehmen zu führen, ferner Knüwer (Hochschulen, 1998): Hochschulen machen das Management spielend einfach. Immer mehr Hochschulen setzen deshalb auf Planspiele, um den Management-Nachwuchs auf die Praxis vorzubereiten.

10 Vgl. hierzu Abb. 1: Funktionen der Logistik und Organisationsformen der Logistik. 
Das dritte Kapitel widmet sich Unternehmenssimulationen. Nach einem kurzen Einblick in die historische Entwicklung wird auf Anwendung und Lernziele von Unternehmenssimulationen eingegangen. Planspiele, wie man Unternehmenssimulationen auch nennt, eignen sich besonders für die Aus- und Weiterbildung, da die in der theoretischen Ausbildung unvermeidbaren Mängel ausgeglichen werden. Durch ein Planspiel lassen sich die in der Ausbildung erworbenen Kenntnisse leichter in praktische Fähigkeiten umsetzen. Deshalb sollen zunächst Aufbau und Funktionsweise von Planspielen näher dargelegt werden. Es folgen die Beschreibung von Merkmalen für die Einteilung von Planspielen und eine Klassifizierung in generelle, funktionelle und institutionelle Planspiele. Dabei werden jeweils zwei existierende Planspiele kurz dargestellt.

Das vierte Kapitel stellt das Unternehmensmodell LogisticPLUS und damit die Produktionsstruktur der simulierten Unternehmung vor. Am Anfang wird auf die Grundlagen und das Umfeld eingegangen. Danach stehen das simulierte Unternehmen sowie die Markt- und Wettbewerbersituation im Vordergrund. Das Unterkapitel endet mit Hinweisen sowohl auf das den Markt simulierende Spielleitermodul als auch auf die ausführbaren Computerprogramme.

Anschließend erfolgen eine Beschreibung des Leistungsflusses des Unternehmens sowie ein Überblick über die Absatzmärkte. Ferner werden die Entscheidungsbereiche Marketing, Produktion, Logistik, Qualitätssicherung, Finanzierung und Personalwirtschaft vorgestellt und Verknüpfungen untereinander aufgezeigt. Diese Definitionen sind wichtige Grundlagen für die 52 durch die Spieler zu treffenden Entscheidungen. Die Größenordnung der Anzahl der Entscheidungsvariablen ist dabei ein guter Kompromiss aus Detailgenauigkeit in der Modellierung der Wirklichkeit und dem für die Spieler notwendigen Überblick und Funktionsverständnis.

Die Auswirkungen dieser Entscheidungen schlagen sich im Rechnungswesen nieder. Es folgen deshalb Ausführungen $\mathrm{zu}$ Gewinn- und Verlustrechnung, Bilanz und Finanzkennzahlen wie Liquidität, Cashflow und Eigenkapitalrentabilität. Ferner werden relevante logistische Betriebskennzahlen aus den Bereichen Umlaufvermögen, Lagerhaltung und Transporte beschrieben. Außerdem werden die für die 
Entscheidungsfindung wichtigen Parameter wie beispielsweise Marktpreise oder eventuell beauftragte Marktforschungsergebnisse vorgestellt.

Auf das Spielleitermodul geht das fünfte Kapitel ein. In diesem Modul, das ebenfalls Bestandteil der Arbeit und somit auch programmiert worden ist, kann die Spielleitung die Märkte beeinflussen: So sind z.B. Marktvolumina für die beiden Produkte oder Preise für Produktionsfaktoren für jede Periode festzulegen. Das Modul eignet sich gut für den Spielleiter, um einen Überblick über jeden einzelnen der fünf Spieler und über die Märkte zu erhalten. Diese Informationen sind einerseits Grundlage für die periodisch zu erstellenden Marktberichte der Spielleitung. Mit ihrer Hilfe werden die Spieler über anstehende Ausschreibungen, Marktentwicklungen oder auch Preisveränderungen informiert. Andererseits dient dieses Modul für das Einzel-Feedback für jeden Spieler.

Das sechste Kapitel beschäftigt sich mit Inhalten der Lehre, die spielbegleitend unterrichtet werden müssen. Nach einer Einführung und Zielgruppenidentifikation werden die Einsatzmöglichkeiten von LogisticPLUS dargestellt. Damit die Spieler richtige Entscheidungen treffen können, werden im Anschluss daran die Wissensbereiche identifiziert, die für Entscheidungsfindung notwendig sind. Diese Bereiche werden in einem ersten Vorschlag zu Lehrmodulen zusammengefasst, welche je nach Einsatzart des Planspiels vorab oder parallel gelehrt werden sollten.

Im siebten Kapitel werden die ersten Ergebnisse des Planspiels erörtert. Die zentralen Fragestellungen werden zusammenfassend beantwortet. Zusätzlich wird auf zukünftige Forschungsfelder hingewiesen.

Um einen kurzen Einblick in die Programmiersprache ,Visual Basic for Applications' von Microsoft zu erhalten, sind im Anhang zwei kurze Ausschnitte aus dem Programmcode des Planspiels abgedruckt. 


\title{
2 Logistik in Theorie und Praxis
}

\author{
The work of logistics takes place across a \\ broad geographical playing field twenty- \\ four hours a day, seven days of every \\ week. 11
}

\subsection{Grundlagen der industriellen Logistik}

Der Begriff Logistik stammt ursprünglich aus dem militärischen Bereich. Er wurde insbesondere für Nachschubgestaltung und Truppenbewegungen verwendet und dadurch bis heute geprägt. ${ }^{2}$ Mitte der sechziger Jahre wurde dieser Begriff in den USA auch für zivile Bereiche übernommen.

Wie für das Militär besteht auch für ein Industrieunternehmen der logistische Auftrag darin, die richtige Menge der richtigen Objekte als Gegenstände der Logistik (Güter, Personen, Energien, Informationen) am richtigen Ort (Quelle, Senke) im System, zum richtigen Zeitpunkt, in der richtigen Qualität und zu den richtigen Kosten zur Verfügung zu stellen.

Logistik hat sich gegen Ende des 20. Jahrhunderts zunehmend zu einem Schlagwort und viel beachtetem Begriff entwickelt. Bei verschiedenen Autoren und Verbänden ist selten ein übereinstimmender Begriffsinhalt festzustellen. Ausdrücke wie Beschaffung, Einkauf, Materialwirtschaft, Logistik und ganz aktuell Supply-Chain-Management

1 Bowersox et al. (Logistical Excellence, 1992) Seite 12.

12 Gemäß „Handbuch der Logistik“ der Deutschen Bundeswehr ist Logistik die „Lehre von der Planung, der Bereitstellung und vom Einsatz der für militärische Zwecke erforderlichen Mittel und Dienstleistungen zur Unterstützung der Streitkräfte oder die Anwendung dieser Lehre“. Vgl. Bundeswehr (Logistik, 1982). Die Bundeswehr nennt als Funktionen der Logistik die Materialwirtschaft (Einführung des Materials, Materialplanung, Materialbedarfsdeckung, Materialbewirtschaftung, Materialerhaltung), das Verkehrs- und Transportwesen sowie die Unterstützung des Sanitätsdienstes. Vgl. Bundeswehr (ZDv 30/41, 1984).

13 Jünemann (Materialfluss, 1989), Seite 11 und 18. Vgl. hierzu auch Bowersox (Logistical Management, 1978) Seite 4, Weber (Logistik-Controlling, 1990) Seite 9, oder auch Wildemann (Logistik, 1992) Seite 192. 
bezeichnen insbesondere in der Praxis sowohl Funktionen im Sinn von betrieblichen Aufgaben als auch Organisationsformen (z.B. Abteilungen). An dieser Stelle mag es genügen, drei Definitionen vorzustellen. ${ }^{14}$

Der erste Definitionsansatz kann als flussorientierte Definition der Logistik bezeichnet werden: „Zur Logistik gehören alle Tätigkeiten, durch die die raum-zeitliche Gütertransformation und die damit zusammenhängenden Transformationen hinsichtlich der Gütermengen und -sorten, der Güterhandhabungseigenschaften sowie der logistischen Determiniertheit der Güter geplant, gesteuert, realisiert oder kontrolliert werden. Durch das Zusammenwirken dieser Tätigkeiten soll ein Güterfluss in Gang gesetzt werden, der einen Lieferpunkt mit einem Empfangspunkt möglichst effizient verbindet.“

Ein zweiter Definitionsansatz kann als lebenszyklusorientierte Definition der Logistik bezeichnet werden. Sie baut auf dem Lebenszyklus eines Erzeugnisses im Sinne seiner Lebensdauer auf. Dem Begriff des Lebenszyklus liegt der Gedanke zugrunde, dass ein Produkt - allgemeiner ein System - durch Maßnahmen der Planung, des Entwurfs und der Entwicklung entsteht und nach einer Periode des Betriebs schließlich stillgelegt oder verschrottet wird.

Ein dritter Definitionsansatz kann schließlich als dienstleistungsorientierte Definition der Logistik bezeichnet werden. Sie baut auf dem Gedanken auf, dass eine Dienstleitung einem Kunden nur optimal zur Verfügung gestellt werden kann, wenn alle Aktivitäten zur Produktion in koordinierter Weise erbracht werden. Der Schwerpunkt dieser Aktivitäten liegt in den folgenden drei Gebieten: Minimierung der Wartezeiten (der Auftragsabwicklungszeiten), Management der Dienstleistungskapazität und Bereitstellung der Dienstleistung durch einen Distributionskanal.

Im nachfolgenden wird eine flussorientierte Definition der Logistik gewählt: „Logistik ist der Teil der Wertschöpfungskette, der die Planung, Realisierung und Kontrolle des effizienten, kosteneffektiven Fliessens und Lagerns von Gütern und Services und der

14 Vgl. hierzu und im nachfolgenden Pfohl (Logistiksysteme, 2000) Seite 12 ff. 
damit zusammenhängenden Informationen vom Liefer- zum Empfangspunkt entsprechend des Anforderungen des Kunden abdeckt.“ Diese Definition stammt von der US-amerikanischen Logistikgesellschaft ,Council of Logistics Management ‘ und ist insbesondere in den USA weit verbreitet.

In diesem Zusammenhang ist die Materialwirtschaft von der Logistik abzugrenzen. Unter der klassischen Materialwirtschaft versteht man heute neben der Funktion Einkauf die Funktionen Materialbereitstellung, (innerbetrieblicher) Transport und Lagerhaltung. Sie umfasst alle Vorgänge der Bewirtschaftung von Erzeugnis- und Betriebsstoffen, unabhängig davon, für welche betrieblichen Teilbereiche diese vollzogen werden. Diese Beschränkung auf die Sicherstellung der Leistungsbereitschaft führt aufgrund vielfältiger Interdependenzen zwischen den Transformations- und Transferprozessen entlang den Wertschöpfungsketten zwangsläufig zu suboptimalen Problemlösungen. Die Erweiterung um die Produktionsplanung und -steuerung sowie um die Versorgung des Marktes wird in der Integrierten Materialwirtschaft berücksichtigt. Diese ist durch alle Aufgaben der Materialwirtschaft bestimmt, die Bestände bzw. den Materialfluss determinieren.

5 Vgl. hierzu auch: Arthur D. Little (Logistics, 1991) Seite 34 ff.

16 Im Original: „Logistics is that part of the supply chain process that plans, implements, and controls the efficient, effective flow and storage of goods, services, and related information from the point of origin to the point of consumption in order to meet customers' requirements." Übersetzung durch den Verfasser, vgl. auch http://www.clm1.org. 


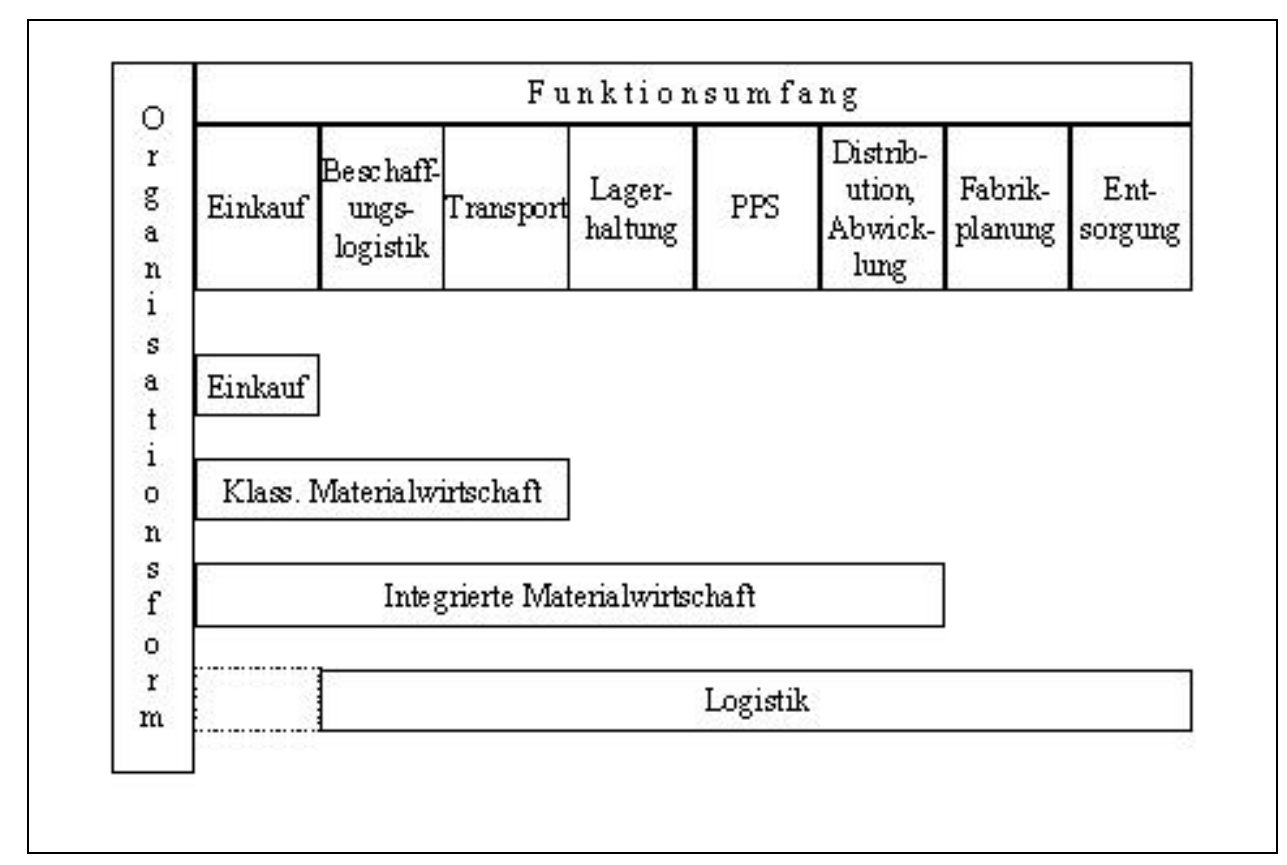

Abb. 1: Funktionen der Logistik und Organisationsformen der Logistik

Klar erkennbar ist der übergreifende Inhalt des Begriffes Logistik. So werden im Unterschied zur Materialwirtschaft auch die Funktionen Fabrikplanung und Entsorgung mit erfasst. Die durch die ganzheitliche Betrachtungsweise des gesamten Güterflusses notwendige Abstimmung der verschiedenen Unternehmensbereiche zur Vermeidung von suboptimalen Insellösungen stellt das eigentliche Rationalisierungspotenzial der Logistik dar.

Logistik bildet eine Querschnittsfunktion und ist deshalb von strategischer und operativer Bedeutung. 19 Zum einen wird die Logistik als ein Schlüsselfaktor für das Erreichung bestimmter strategischer Ziele angesehen. So sind z.B. bei der Verfolgung der Strategie der Kostenführerschaft oftmals Logistiksysteme notwendig, die den Transport relativ großer Gütermengen in weiträumigen Marktgebieten mit geringen Kosten ermöglichen. Zur Kostensenkung können eine Vielzahl von Strategien und Maßnahmen zur Rationalisierung der Logistik beitragen, z.B. die Automatisierung der

17 Schulte (Logistik, 1995) Seite 2.

18 Harlacher (Logistikkostenrechnung, 1988) Seite 272.

19 Vgl. Götze / Mikus (Strategisches Management, 1999) Seite 247 ff. Vgl. auch Klöpper (Management, 1991) Seite 43: „Der operative Erfolg von Unternehmungsstrategien ist zunehmend abhängig von einem leistungsfähigen Logistiksystem. Insbesondere dort, wo die Zeit als eine kritische Erfolgsgröße zählt, gewinnt die Logistik an strategischer Relevanz.“ 
Materialflusstechnik oder die Reduzierung von Beständen. Bei einer Strategie der Differenzierung kann ein hoher Lieferservicegrad ein Differenzierungsmerkmal darstellen. Die Erreichung eines besonderen Kundennutzens über die Logistikleistungen setzt die Flexibilität des Logistiksystems voraus. Die Strategie der Konzentration auf Schwerpunkte impliziert eventuell eine räumliche Beschränkung des zu versorgenden Marktes und damit gegebenenfalls einfachere Anforderungen an die Logistik oder aber die Notwendigkeit des Anpassung des Logistiksystems an die spezifischen Bedürfnisse in einem Marktsegment. ${ }^{60}$ Zum anderen hängt die operative Tagesarbeit zu einem großen Teil von der zeitgerechten Bereitstellung der richtigen Güter am richtigen Platz $\mathrm{ab}$.

Dem Aspekt der strategischen und operativen Querschnittsfunktion wird derzeit vermehrt in der produzierenden Industrie Rechnung getragen. Dies äußert sich darin, das sich bisher stark nach Abteilungen strukturierte Unternehmungen in eine Produktionsgesellschaft und eine Vertriebsgesellschaft aufteilen. ${ }^{2}$ Dabei umfasst der technische Bereich in der Regel die Abteilungen Produktion, Materialwirtschaft, Qualitätssicherung 22 sowie Forschung \& Entwicklung. Der vertriebliche Bereich setzt sich aus den Abteilungen Marketing, Vertriebsinnen- und Außendienst, Versand Produktmanagement, Kundenbetreuung (Anwendungstechnik) und Reklamationsbearbeitung zusammen.

Die Logistik wirkt sich auf beide Bereiche aus. So ist die Leistungserbringung fast aller technischen Bereiche unmittelbar mit der Bereitstellung der Produktionsfaktoren verknüpft. Die vertrieblichen Abteilungen benötigen ebenfalls die Logistik: Ohne die zeitgerechte Bereitstellung bzw. Auslieferung der Produkte ist eine verspätete bzw. keine Rechnungsstellung möglich, oder: Die Reklamationsabteilung muss den zeitnahen und sachgerechten Ersatz von Produkten gewährleisten. Störungen in der Logistik verursachen häufig Störungen in anderen Bereichen.

20 Götze / Mikus (Strategisches Management, 1999) Seite $247 \mathrm{f}$.

21 Diese Gesellschaften können eine eigene Rechtsform haben, müssen aber nicht. Oftmals wird jede von beiden von einer eigenständigen Geschäftsführung geführt.

22 Qualitätssicherung wird hier als rein technische Funktion, d.h. Produkt- bzw. Warenkontrolle angesehen. 
Dies führt zum naheliegenden Zielsystem der Logistik:

\begin{tabular}{|l|l|}
\hline Erhaltung der Wettbewerbsfähigkeit & oberste \\
Erzielung eines befriedigenden Gewinns & Unternehmensziele \\
\hline Sicherung der Beschaffungsmärkte & \\
Sicherung der Lieferbereitschaft & langfristige \\
Sicherung der Qualität und des Technologiestatus & Unternehmensziele \\
Erschließung von Kosten- und Bestandssenkungspotenzialen & \\
\hline Optimierung der Materialkosten & \\
Optimierung der Kostenwirtschaftlichkeit & \\
Optimierung der Kapazitätsauslastung & mittelfristige \\
Optimierung des Servicegrades & Unternehmensziele \\
Optimierung der Materialqualität & \\
Optimierung der Kapitalbindung & \\
\hline
\end{tabular}

Tab. 1: Zielsystem der Logistik 24

Es ist ersichtlich, dass die obersten und langfristigen Unternehmensziele strategische Überlegungen beinhalten, während die mittelfristigen Ziele eher operativer Natur sind.

Beispiele für strategische Überlegungen sind Einkaufallianzen, Lieferantenauswahl oder generelle Beschaffungsstrategien. Operative Funktionen sind z.B. die Durchführung der Wareneingangskontrolle mit definierten Prüfplänen, der automatische Warenumschlag oder die Automatisierung der Abrufe mittels eines PPS-Systems ${ }^{65}$.

Von den logistischen Funktionen üben die Lagerhaltung und die Transporte starken Einfluss auf das Ergebnis eines Unternehmens aus. Hier sind insbesondere die Kapitalbindungskosten zu nennen, die sich sowohl in der Produktion als auch in der Lagerhaltung direkt auswirken. 26 Durch das nachfolgende Beispiel, das den Einfluss der Lagerveränderungen auf den ROI zeigt, lässt sich die Bedeutung der Kapitalbindung im Umlaufvermögen erklären.

23 Der Versand könnte selbstverständlich auch zum technischen Bereich zugeschlagen werden. Grundlage für eine Entscheidung der Zuordnung könnte z.B. die Wertigkeit der Transportleistung für den Kunden sein.

24 Engel (Konzernlogistik, 1994).

25 Ein PPS-System hat die Aufgabe, aufgrund erwarteter und vorliegender Kundenaufträge, den Material- und Warenfluss in den Produktionsstellen zielgerichtet nach Art, Menge und Zeit für den Planungszeitraum unter Beachtung betrieblicher Ziele festzulegen, diese Vorgaben durchzusetzen, die Auftragsabwicklung zu überwachen und bei Abweichungen Maßnahmen zu ergreifen. Vgl. Zäpfel (Produktionsplanung, 1993) Seite 26. 


\begin{tabular}{|c|c|c|c|c|}
\hline $\begin{array}{c}\text { Umsatz } \\
\text { [Mio. €] }\end{array}$ & $\begin{array}{c}\text { Lagerbestand } \\
\text { [Mio. } € \text { ] }\end{array}$ & $\begin{array}{c}\text { zusätzliche Zinsen } \\
\text { absolut }\end{array}$ & $\begin{array}{c}\text { Rendite } \\
\text { absolut }\end{array}$ & $\begin{array}{c}\text { ROI } \\
{[\%]}\end{array}$ \\
\hline 10 & 2 & 0,2 & 0,4 & 8,0 \\
\hline 10 & 3 & 0,3 & 0,3 & 5,0 \\
\hline 10 & 4 & 0,4 & 0,2 & 2,9 \\
\hline 10 & 5 & 0,5 & 0,1 & 1,3 \\
\hline
\end{tabular}

Tab. 2: Einfluss des Lagerbestandes auf den ROL

Deutlich erkennt man, dass ein erhöhter Lagerbestand bei gleichbleibendem Umsatz aufgrund von erhöhten Zinsaufwendungen eine geringere absolute Rendite und damit einen geringeren ROI bewirkt.

Im Schrifttum finden sich zahlreiche Erfolgsfaktoren der Logistik. Schulte beschreibt die Erfolgsfaktoren für exzellente Logistik, die in einer Studie im Auftrag der National Association of Accountants und des Council of Logistics Management genannt werden: 28

1. Verknüpfung der Logistik mit der Unternehmensstrategie: Logistik darf nicht als neutrales Element oder als notwendiges Übel, sondern ist als wesentlicher Erfolgsfaktor anzusehen.

2. Ganzheitliche Organisation: Es muss eine definierte Struktur bzw. Organisation geben, die sämtliche Funktionen (Produktion, Logistik, Vertrieb, etc.) miteinander verbindet. Klare Zuständigkeiten und Entscheidungsspielräume müssen definiert sein. Alle logistischen Funktionen sind in einer Organisationseinheit zusammenzufassen.

3. Umfassende Nutzung von Informationen und Informationssystemen: In der Praxis zeigt sich oft, dass z.B. bei verteilter Produktion an mehreren Standorten unterschiedliche Software existiert. So wird die Beschaffung oder das PPS-System auf verschiedenen Plattformen ausgeübt, was einen Informationsaustausch schwierig und langsam gestaltet. Information muss als strategische Ressource erkannt werden.

26 Vgl. hierzu Wildemann (Logistik, 1992) Seite 193 oder auch Hug (Beständecontrolling, 1988) Seite259: Seit einiger Zeit lässt sich allerdings feststellen, dass die Höhe der vorgehaltenen Bestände immer öfter einer kritischen Überprüfung unterzogen wird, um die Vorräte zu reduzieren und damit die für deren Bereithaltung anfallenden Kapital- und Lagerkosten zu senken.

27 Reutner (Strategietagung, 1995) Seite 85.

28 Vgl. Schulte (Logistik, 1995) Seite 403 ff. 
4. Betonung der Humanressourcen: In der Unternehmung sind erfahrene, gut ausgebildete Logistikmanager für die erfolgreiche Umsetzung der Logistikstrategien entscheidend. Hier treten aufgrund des knappen Arbeitsmarktangebotes an erfahrenen Logistikern gern Engpässe auf. On-the-job training wird immer weniger als hinreichend für die Aufgabenbewältigung im Logistikbereich angesehen. Mitarbeiter des Logistikbereiches sollten verstärkt an externen Weiterbildungsveranstaltungen teilnehmen, um beispielsweise neue Konzepte und Methoden kennen zu lernen.

5. Bildung strategischer Allianzen: In der Automobilindustrie werden erste Beschaffungs- und Konsolidierungszentren mit Erfolg betrieben, weshalb auch andere Branchen diesen Weg zunehmend gehen. 29

6. Fokussierung auf finanzielle Ergebnisse: Es müssen aussagekräftige finanzielle Indikatoren als Maßstab für die Logistikeffizienz existieren. Es zeigt sich, dass die Funktionen Transport, Lagerhaltung und Kundendienste am effizientesten gesteuert werden können, wenn sie als cost- oder profit-center organisiert sind. Auf diese Weise wird das unternehmerische Engagement der Logistikmanager gefördert.

7. Festlegung optimaler Serviceniveaus: Zur Optimierung des Ergebnisbeitrages der Logistik sollte man die Kundenbedürfnisse und -erwartungen bezüglich des Servicegrades kennen. Unternehmen müssen das optimale Serviceniveau und die damit einhergehenden Kosten ermitteln.

8. Aufmerksamkeit für Details: Die besten Logistikorganisationen haben die Basisabläufe voll im Griff und arbeiten ständig an scheinbar kleinen Problemen. In ihrer Gesamtheit führen dieses Lösungen zu hervorragenden Leistungen. ${ }^{30}$ Ein Schlüssel zur Vereinfachung der Abläufe liegt in der Erschließung des Wissens sowie in der Erfahrung und Kreativität der Mitarbeiter in der Linie.

9. Zusammenfassen von Logistikmengen: Dass Zusammenfassen von Sendungen oder sonstigen wiederkehrenden Aktivitäten $\mathrm{zu}$ Kosteneinsparungen führt, ist hinlänglich bekannt. Voraussetzung zur tatsächlichen Erzielung dieser Effekte ist jedoch eine fundierte Analyse der zur Verfügung stehenden Alternativen und tradeoff-Beziehungen.

29 Miebach / Blauermel (Beschaffungszentrum, 1998).

30 In diesem Zusammenhang sei der Begriff KVP - Kontinuierlicher Verbesserungsprozess erwähnt. Vgl. Hessenberger / Kuhn (KVP, 1996): „KVP: Mit guter Logistik fängt alles an“. 
10. Aktives Controlling: Eine einmal aufgebaute exzellente Logistik wird dies nur auf Dauer bleiben, wenn entsprechende Anstrengungen unternommen werden. Unternehmen müssen deshalb ihre Logistikkosten und -leistungen permanent messen, um kritische Zielabweichungen frühzeitig identifizieren und in Abhängigkeit von den Ergebnissen mit Maßnahmen reagieren zu können.

Im vorliegenden Planspiel werden die maßgeblichen Erfolgsfaktoren berücksichtigt. Aus Gründen der Praktikabilität liegt der Schwerpunkt von LogisticPLUS auf den oben beschriebenen Punkten (2) Ganzheitliche Organisation, (3) Umfassende Nutzung von Informationen und Informationssystemen, (4) Betonung der Humanressourcen, (6) Fokussierung auf finanzielle Ergebnisse und (8) Aufmerksamkeit für Details. Wie erwähnt ist es das Ziel des Planspiels, dem Logistikpraktiker die Auswirkungen seiner logistischen Entscheidungen auf den wirtschaftlichen Erfolg der gesamten Unternehmung aufzuzeigen. Er soll die Zusammenhänge zwischen Marketing, Absatz und Logistik schneller erkennen und damit eine Verbesserung der logistischen Entscheidungen erreichen können.

\subsection{Logistische Funktionen und ihre Aufgaben}

Moderne Logistiksysteme und -aktivitäten stellen an die Verantwortlichen hohe Anforderungen. Logistiker müssen in der Lage sein, ein breites betriebswirtschaftliches Instrumentarium zu beherrschen und einzusetzen. Dazu gehören zunächst die direkten Logistikkomplexe bzw. -arten: Beschaffungslogistik, Produktionslogistik, Distributionslogistik und Entsorgungslogistik. 


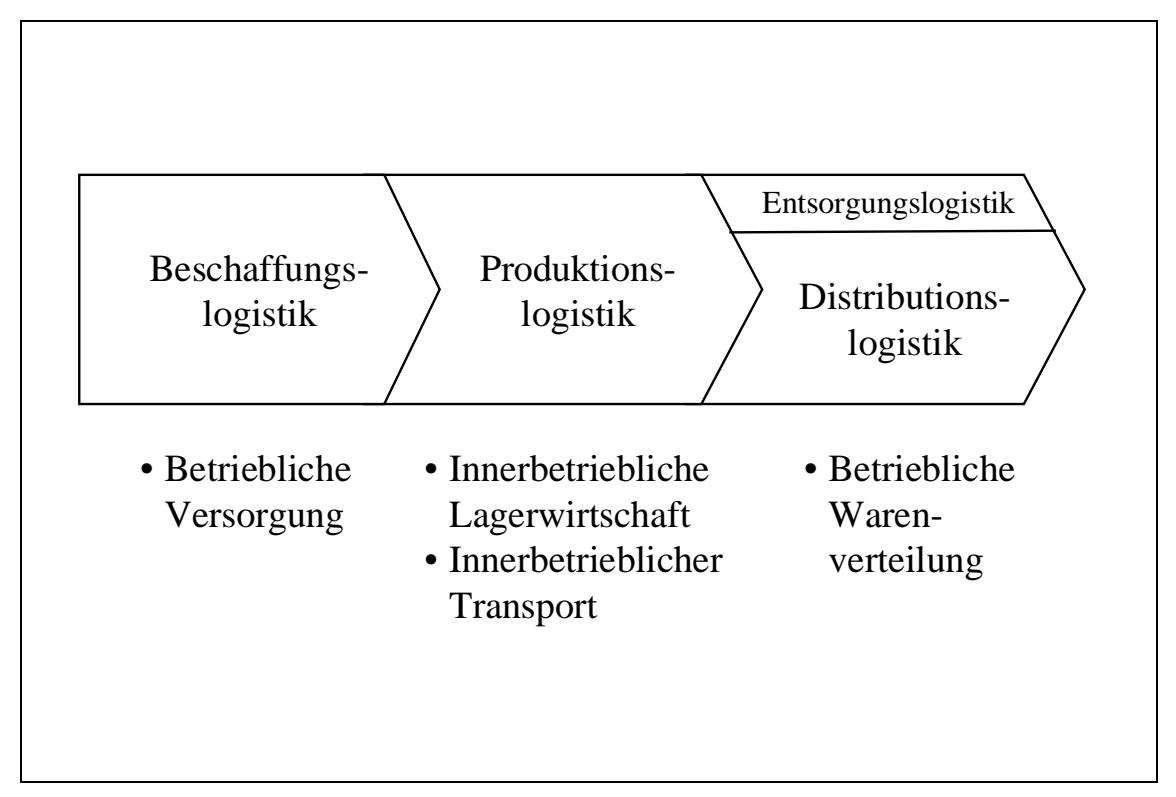

Abb. 2: Logistische Funktionen

Die einzelnen Funktionen werden im folgenden kurz beschrieben.

\subsubsection{Beschaffungslogistik}

In der Realität kommt der sorgfältigen Planung der Unternehmen auf dem Gebiet der Beschaffungslogistik eine nicht $\mathrm{zu}$ unterschätzende Bedeutung $\mathrm{zu}$. Von jeder benötigten Materialart die richtige Menge zum richtigen Zeitpunkt am richtigen Ort zu haben, ist sowohl für die Produktions- als auch für die Vertriebsgesellschaft eine der Grundlagen erfolgreichen wirtschaftlichen Handelns. 13

Auf den Logistiker kommen hier Aufgaben $z u$ wie Bedarfsermittlung, Lieferantenbeziehungen, Projektplanung, Einkaufsprogramme, Beschaffungswege, Make- or Buy- Entscheidungen, Dienstleistungseinkauf, Outsourcing, Simultaneous Engineering, Markterkundung, Zuteilung, Liefereinteilung oder Gewährleistungsverfolgung. 
In einer jüngeren Veröffentlichung wird insbesondere auf den Zusammenhang zwischen

Beschaffungsentscheidungen und Logistik hingewiesen. So stellen Pfohl und Schäfer fest, dass sowohl operative, in konkreten Beschaffungsfällen $\mathrm{zu}$ treffende Entscheidungen als auch strategische, d.h. langfristige Entscheidungen im Beschaffungsbereich erheblichen Einfluss auf die logistischen Prozesse bei der Güterbeschaffung haben können. Bei der Entscheidungsfindung selbst werden logistische Aspekte häufig noch immer nur in geringem Umfang berücksichtigt. Der Grund liegt darin, dass entweder entsprechende Daten- und Informationsgrundlagen fehlen, weil Entscheidungsbeteiligten aus dem Logistikbereich kein ausreichender Einfluss auf den Entscheidungsprozess eingeräumt wird, oder viele Prozesse routinemäßig ablaufen, ohne näher hinterfragt $\mathrm{zu}$ werden. Hier sind kritische Überprüfungen vor allem der unternehmensinternen Abläufe im Rahmen von Beschaffungsprozessen angezeigt.

Auch der in Frankfurt ansässige Bundesverband Materialwirtschaft, Einkauf und Logistik (BME) weist in einer aktuellen Veröffentlichung auf die Auswirkungen der Beschaffung hin: Guter und kostengünstiger Einkauf trage mehr zum Unternehmenserfolg bei als Marketing und Vertrieb. Ein Prozent Ersparnis im Einkauf potenziert sich auf den Gewinn mit 10 Prozent, ein Prozent Umsatzwachstum steigert ihn hingegen nur um 0,1 Prozent. Die Aufgaben der Beschaffung würden nach Ansicht des Bundesverbandes Materialwirtschaft, Einkauf und Logistik in den kommenden Jahren stark wachsen; der elektronische Handel werde das Geschäft revolutionieren. 34

\subsubsection{Produktionslogistik}

Die Produktionslogistik umfasst einen Teil der Losgrößendefinition und die Bereiche Materialdisposition und Lagerwirtschaft. Neben der zeitgerechten Bereitstellung der

33 Pfohl / Schäfer (Beschaffungsprozess, 1999) Seite 252.

34 FAZ (19. August 2000 A) Seite 59. Ähnlich äußert sich auch Hoffmann: In den meisten (Industrie-) Unternehmen werden etwa 50 Prozent des Gesamtumsatzes in Form von Rohmaterialien, Fertigteilen, Halbzeugnissen oder Dienstleistungen usw. durch die Einkaufsabteilung in das Unternehmen gebracht. Die Materialwirtschaft eines Unternehmens, das einen 200 Millionen Euro Umsatz verbucht, ist für den Einkauf von Gütern in Höhe von ca. 100 Millionen verantwortlich. Eine Einssparung im Einkauf von nur 5 Prozent würden den Gewinn des Unternehmens um 5 Mio. Euro pro Jahr erhöhen. Vgl. Hoffmann (Wertanalyse, 1994) Seite 169. 
Produktionsfaktoren wird die optimale Bestandsführung einerseits wegen der direkten und oftmals großen Abhängigkeit für die Kostenstruktur, andererseits wegen reduzierter Absatzmengen aufgrund vermehrt vorkommender verkürzter Lebenszyklen immer wichtiger.

So befinden sich viele Unternehmen in einem Strukturwandel zum variantenreichen Serienfertiger mit heterogener Auftrags- und Stückzahlcharakteristik. Eine Vielzahl von Komponenten und Einzelteilen in einer komplexen, vernetzten Produktionsstruktur stellten höchste Anforderungen an die Effizienz der Auftragsabwicklung. Ursprünglich erzielte Produktivitätssteigerungen durch Automatisierung direkter und indirekter Einzelfunktionen werden zunehmend durch steigenden Lenkungsaufwand aufgezehrt.

In diesem Zusammenhang seien beispielhaft die Ablauf- / Reihenfolgeplanung erwähnt. Ziel ist es, das Dilemma der konkurrierenden Zielbeziehung zwischen den ablauforganisatorischen Zielsetzungen der Minimierung der (mittleren) Durchlaufzeit der Aufträge (und damit auch des Materials) und der Maximierung der Kapazitätsauslastung des Produktionssystems zu lösen. 36

In den Bereich der Produktionslogistik fällt auch die innerbetriebliche Standort- bzw.

Layoutplanung. Die hier anfallen Probleme lassen sich idealtypischerweise in vier Bereiche unterscheiden:

1. Grundstücks-Layout-Probleme: Hierunter fallen Fragen zur Lage von Gebäuden (Fabrik- und Lagerhallen, Verwaltungsgebäude) sowie von unbebauten Nutzflächen (Freiluftlager, Parkplätze) auf dem Betriebsgrundstück.

2. Gebäude-Layout-Probleme: Gewisse Funktionsbereiche (Fertigung, F\&E, Lagerhaltung, Verwaltung, usw.) müssen sinnvoll angeordnet werden.

3. Funktionsbereichs-Layout-Probleme: Hier gilt es $\mathrm{zu}$ lösen, wo z.B. Qualitätskontrolle, die zentrale Instandhaltung oder Produkt-, Material-, Werkzeugoder Ersatzteillager vorzusehen sind

\footnotetext{
Vgl. Pawellek / Hartmann (Produktionslogistik, 1999) Seite $253 \mathrm{ff}$.

Vgl. Pfohl (Logistiksysteme, 2000) Seite 198.

Vgl. Wäscher ( Layout, 1993), Seite 78.
} 
4. Abteilungs-Layout-Probleme: Auf der nächstniedrigeren Aggregationsstufe stellt sich die Frage, wo z.B. Schreibtische aufzustellen oder Produktarten im Lager positionieren sind.

Aufgaben für den Logistiker sind hier z.B. der Bedarf an Halbfabrikaten (programmoder verbrauchsgesteuert), die Festlegung von Anforderungsmengen und -zeitpunkten, die Bestands- und Bewegungskontrolle, der Abruf und die Terminüberwachung.

\subsubsection{Distributionslogistik}

Die Schließung der räumlichen, zeitlichen, art- und mengenmäßigen Lücke zwischen Produktion und Konsumtion übernehmen die Lager-, Transport- und sonstigen Leistungen der Distributionslogistik, die für eine optimale Bedienung der Absatzwege und damit der Versorgung der Kunden erforderlich ist. ${ }^{39}$ Die Gestaltung der Absatzwege hängt maßgeblich von der Distributionsstruktur und -strategie eines Unternehmens ab.

Beispiele:

1. Postversand: Diese (externe) Dienstleitung eignet sich insbesondere für Bücher, Computer oder Datenträger wie CDs. Eine Bestellung, die beispielsweise über das Internet aufgegeben wurde, wird in einem logistischen Verteilzentrum bearbeitet bzw. abgewickelt. Dabei ist der Server, der die Bestellung entgegen nimmt, in der Regel nicht am Ort des Verteilzentrums. Dieses liegt an einem optimalen Standort: Kurze Wege zum Kunden (z.B. durch Anbindung an ein Postverteilungszentrum), gute Anbindung an Verkehrswege (für die Anlieferung der $\mathrm{zu}$ versendenden Produkte) sowie günstige Personal- und Strukturkosten.

2. Lieferservice: Hierunter versteht man eine selbst erbrachte Dienstleistung, wie sie

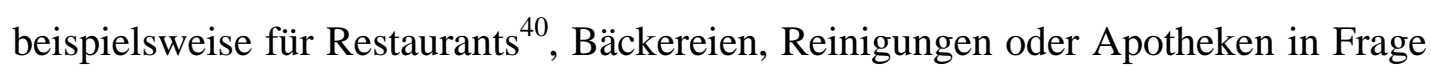
kommt. Ab einem bestimmten Umsatz liefert der Hersteller oder Dienstleister seine Produkte kostenfrei zum Kunden. Diese Art ist insbesondere für die regionale bzw. städtische Verteilung geeignet.

\footnotetext{
38 Vgl. dazu Bloech / Rottenbacher (Materialwirtschaft, 1986) Seite 9 oder auch Bloech et al. (Produktion, 1998) Seite $176 \mathrm{ff}$

39 Kleeberg (Transportnetzwerke, 2000) Seite 13.
} 
3. Standardisierter (Einzel-) Güterverkehr auf der Straße, der Schiene oder in der Luft: Dieser eignet sich z.B. für die regelmäßige Belieferung von Händlern. So werden fabrikneue Autos über Bahn oder LKW zum Händler transportiert oder der Großhandel durch die Produktions- oder Lebensmittelindustrie angefahren. Der Transport von Großgeräten für Baustellen wird mit Flugzeugen, Schiffen, Eisenbahn oder LKW erledigt.

4. Bei der Selbstabholung wird die Distribution durch den Hersteller in eine Abholung durch den (End-) Kunden substituiert. Hierfür bieten sich individuelle (Design-) Produkte, wie Brillen, Kleidung oder Automobile, aber auch Produkte des täglichen Bedarfes, wie Lebensmittel oder Baumarktprodukte, an. Unter die Selbstabholung fällt auch das „Abholen“ einer ärztlichen Leistung in einer Praxis oder Klinik. Die Alternative, und damit vergleichbar mit Punkt 2 „Lieferservice“, wäre der Hausbesuch eines Arztes. 1.1

In jüngster Zeit, insbesondere in Zusammenhang mit der verstärkten Nutzung des Internets, versuchen viele Dienstleister, die Kunden der klassischen Selbstabholerprodukte für einen Lieferservice zu gewinnen. Dabei spielt der geringere Preis eine große Rolle, denn durch Verzicht der (überflüssigen) Handelsstufe kann die Gesamtmarge geringer ausfallen. Mittel eines erhöhten Service, (z.B. Umtauschgarantie ohne Kosten für die Rücksendung) und der Visualisierung der Produkte im Internet wird versucht, dem Kunden die Scheu zu nehmen, ein unbekanntes Produkt auf sein Risiko zu kaufen.

Für die Distributionslogistik spielen die Transportplanungen eine große Rolle. Außer der eigentlichen Transportdisposition gilt es umfangreiche Rundreiseprobleme (Speditionen und Fuhrpark), die innerbetriebliche Standortwahl, Transportprobleme aus mehreren Lagerhäusern für mehrere Versorgungsorte, Disposition interner und externer Dienstleitungen und Umladeprobleme aller Art zu lösen . .2

40 Z.B. Pizzerias, Catering Service oder auch „Essen auf Rädern“.

41 Der Hausbesuch ist nötig, wenn das Abholen der Dienstleistung durch den Patienten aufgrund seiner Erkrankung nicht möglich ist.

42 Vgl. umfassend z.B. Bloech / Ihde (Distributionsplanung, 1972). 


\subsubsection{Entsorgungslogistik}

Der Aufgabenbereich der Logistik umfasst die Versorgung der Bedarfsträger und deren Entsorgung. Gegenstände der Entsorgung sind Leergüter, Reststoffe und Abfall. Leergut umfasst Verpackungen und Transporthilfsmittel, für die eine Wiederverwendung vorgesehen ist. Reststoffe sind produktions- und konsumtionsbedingte Rückstände, die als Kuppelprodukte anfallen, nicht bezweckt sind und deren Beseitigung aufwendig ist. Sofern die Verwertungskosten die Beseitigungskosten übersteigen, werden Rückstände ökonomisch gesehen zu Abfall. 43

Aufgrund des steigenden ökologischen Interesses gewinnt die Entsorgungslogistik immer mehr an Bedeutung. Eine für Unternehmen und Natur optimale Entsorgung der unverbrauchbaren Rückstände aus der Produktion ${ }^{44}$ in Verbindung mit den gestiegenen Anforderungen durch internationale Normen wie ISO 9000 oder ISO $14000^{45}$ kann sich auf den Wert und damit auf den Aktienkurs eines Unternehmens auswirken. Bezüglich einer optimalen Entsorgung ist der Nachholbedarf allerdings noch groß. So zeigen Untersuchungen der Universität Stuttgart, dass beispielsweise bei der Hausmüllentsorgung erst vereinzelt Fahrzeugsysteme zum Einsatz kommen, die durch Teilautomatisierung die Betriebseffizienz erhöhen. Bei der Entsorgung von Industriebetrieben und der Redistribution von verwertbaren Abfällen (Kreislaufwirtschaft) ist noch keine generelles System erkennbar.

43 Ihde (Transport, 1991) Seite 251.

44 Hierunter fallen neben greifbaren auch nichtgreifbare Abfälle wie z.B. Lärm oder Wärme.

45 ISO - Internationale Standardisierungsorganisation (engl. International Organization for Standardization). Die ISO 9000 ist die Grundvorschrift für Qualitätsstandards, die ISO 14000 für Umweltstandards. Vgl. Kiesel (Logistik, 1999).

46 Vgl. Wehking / Schulz (Fahrzeugkonzepte, 1999) Seite $286 \mathrm{ff}$. 


\subsection{Systeme der industriellen Logistik}

Jedes System lässt sich einerseits in ein übergeordnetes System einordnen und andererseits in eine Anzahl von Subsystemen aufgliedern. Das Gesamtsystem Logistik kann in die folgenden verrichtungsspezifischen Subsysteme zerlegt werden:
1. Auftragsabwicklung
4. Verpackung
2. Lagerhaltung
5. Transport
3. Lagerhaus

\subsubsection{Auftragsabwicklung als übergreifender Prozess}

Der Auftrag ist die Grundlage des Informationsflusses im Logistiksystem. Das dazu nötige Auftragsformular sollte mindestens die nachfolgenden Informationen enthalten:

- Auftragsnummer

- Auftragsdatum

- Kundendaten

- Branche des Kunden

- Verkäufer und Verkaufsgebiet

- Artikelbezeichnung

- Artikeldaten
- Menge des Artikels

- Bruttopreis

- Verkaufsbedingungen

- Transportmittel

- Versandkostenanteil

- Versandanschrift

- Liefertermin

Der Auftrag ist eine wichtige Informationsquelle für andere Unternehmensbereiche. Als externer Auftrag (Kundenauftrag) ist er ein wichtiges Bindeglied für die Distributionslogistik des Lieferanten und die Beschaffungslogistik des Kunden. Als interner Auftrag ist er das Bindeglied zwischen intraorganisatorischen Logistiksystemen, z.B. Produktionslogistik und Beschaffungslogistik, oder zwischen Zentrallager und Außenlager. 
Die Auftragsabwicklung, aus Sicht der Distributionslogistik ,die datenmäßige Bearbeitung und Kontrolle der Aufträge vom Zeitpunkt der Auftragsaufgabe beim Kunden bis zur Ankunft der Sendungsdokumente und Rechnungsdokumente beim Kunden“迆, aus Sicht der Produktionslogistik, „die marktgerechte Steuerung der Material- und Informationsflüsse vom Rohmateriallieferanten bis zum Endkunden“, erfüllt drei Funktionen: Die Gewährleistung eines der Güterbewegung vorauseilenden Informationsflusses, die Gewährleistung eines der Güterbewegung begleitenden Informationsflusses und die Gewährleistung eines der Güterbewegung nacheilenden Informationsflusses.

Die Aufgaben der Auftragsabwicklung erstrecken sich auf

1. Übermittlung der Auftragsinformationen durch Kunden oder Dritte (z.B. Außendienstmitarbeiter oder Handelsvertretungen) über Telefon, Fax, E-Mail, etc.

2. Aufbereitung und Umsetzung, d.h. Anpassung an unternehmensinterne Anforderungen,

3. Zusammenstellung (Kommissionierung im Lager) und Versand sowie

4. Fakturierung (Rechnungslegung).

\subsubsection{Die Lagerhaltung als Grundlage des Geschäftes}

Es ist das natürliche ökonomische Bestreben eines Herstellers, seine Lagerhaltung zu optimieren. Dies entspricht meistens einer Verringerung der aktuellen Bestände und dadurch einer Senkung der Kapitalbindung.

Die Lagerhaltung befasst sich mit jenen Entscheidungstatbeständen, die einen Einfluss auf die Lagerbestände ausüben. Lagerbestände sind Puffer zwischen Input- und OutputFlüssen von Gütern. ${ }^{50}$ Diese Puffer entstehen, sobald sich die zeitliche und sie quantitative Struktur der Input-Flüsse von denen der Output-Flüsse unterscheiden.

\footnotetext{
Pfohl (Logistiksysteme, 2000) Seite $71 \mathrm{ff}$.

Pfohl (Logistiksysteme, 2000) Seite 73.

Nagel (Just-in-Time, 1993) Seite 107.

$50 \mathrm{Zu}$ Lagerhaltungsmodellen vgl. umfassend z.B. Meyer / Hansen (Planungsverfahren, 1996), insbesondere Seite $143 \mathrm{ff}$.
} 
Anders gesagt: Das Lager dient der Überbrückung von zeitlichen Differenzen zwischen Angebot und Nachfrage. ${ }^{51}$ Lagerbestände erweisen sich weiterhin als erforderlich, wenn ein Unternehmen Größendegressionseffekte beim Einkauf, Transport oder bei der Produktion von Gütern nutzen möchte.

Bei der Gestaltung eines Puffers zwischen den Input- und Output-Flüssen sind vier eng verzahnte Fragen zu beantworten:

1. Welches Gut soll gelagert werden?

2. Wie viel soll von einem Gut gelagert werden?

3. Wie viel soll zur Wiederauffüllung des Lagerbestandes bestellt werden ?

4. Wann soll zur Wiederauffüllung des Lagerbestandes bestellt werden ?

Durch die Beantwortung dieser Fragen wird die Höhe der Lagerbestände festgelegt. Dabei ist zu beachten, dass die Summe der entstehenden Kosten minimiert wird. ${ }^{53}$ Die folgende Abbildung zeigt den Zusammenhang zwischen den verschiedenen Bestandteilen des Lagerbestandes.

51 Ein Beispiel ist hier die saisonale Nachfrage nach bestimmten Konsumgütern zur Weihnachtszeit. Vgl. auch Nagel (Just-in-Time, 1993) Seite 107.

52 Pfohl (Logistiksysteme, 2000) Seite 94.

53 Meyer / Hansen (Planungsverfahren, 1996) Seite 143. 


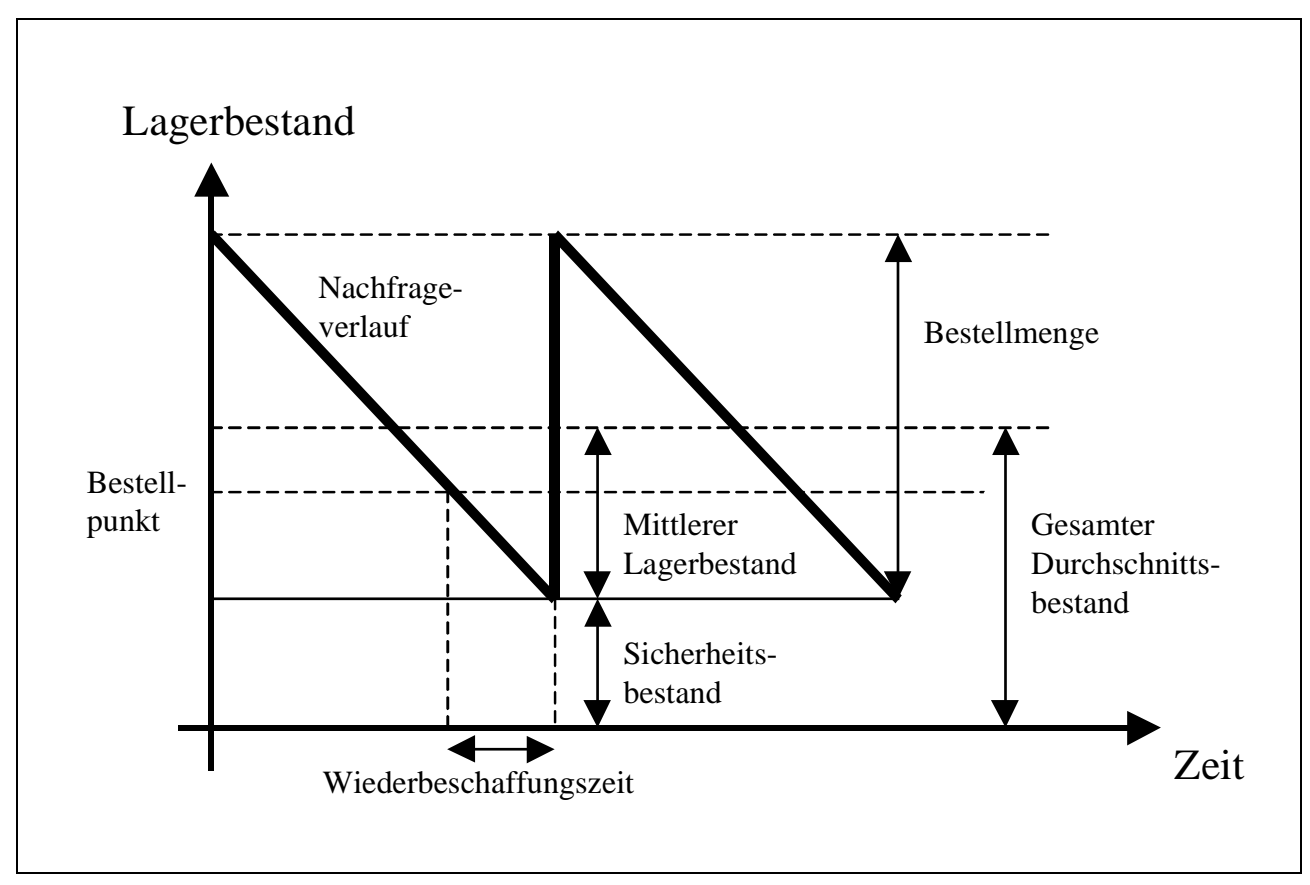

Abb. 3: Komponenten des Lagerbestandes bei Vorratsergänzung und Vorratssicherung

Dieses vereinfachte Modell ist für das Verständnis der Einflussfaktoren auf den Lagerbestand gut geeignet. Um das genannte Ziel - nämlich die Summe der Kosten zu minimieren - zu erreichen, sind die vier obigen Fragen zu stellen. Die Antworten darauf erscheinen auf den ersten Blick trivial: Die Wiederbeschaffungszeit ist in der Regel bekannt, die durch Bestellung und Einlagerung entstehen Kosten sind es ebenfalls. Somit wäre bei linearem Verlauf der Nachfrage die optimale Bestellmenge zu einem bestimmten Zeitpunkt schnell gefunden.

Die in der Theorie getroffenen Annahmen, insbesondere die der Linearität der Nachfrage, sind in der Praxis jedoch nicht der Fall: Das Kundenverhalten ist häufig saisonalen ${ }^{55}$, regionalen oder wettbewerbstechnischen Schwankungen unterworfen. Dies kann zu einer deutlichen Erhöhung oder Senkung der Nachfrage führen. Ebenso ist die Wiederbeschaffungszeit insbesondere bei kleineren Mengen nicht tagesgenau planbar.

54 Vgl. Ihde (Transport, 1991) Seite 192 oder Pfohl (Logistiksysteme, 2000) Seite 95.

55 Beispielhaft seien hier Konsumgüter in der Weihnachtszeit, Nahrungsmittelzutaten für saisonale Produkte (z.B. Spargel), Flüssiggase oder Heizölhandel genannt. 


\subsubsection{Das Lagerhaus als logistisches Zentrum}

Das Lagerhaus ist ein Knoten im logistischen Netzwerk. Lagerhäuser können sowohl Liefer- und Empfangspunkte als auch Auflösungs- und Konzentrationspunkte sein. Prinzipiell kann man drei verschiedene Arten von Lagern unterscheiden: Vorratslager, Umschlaglager und Verteilungslager.

\begin{tabular}{|c|c|c|c|}
\hline Art & wichtigste Funktion & Standort & Lagergüter \\
\hline Vorratslager & hohe Lagerkapazität & $\begin{array}{l}\text { produktions- } \\
\text { orientiert }\end{array}$ & $\begin{array}{l}\text { Material, saisongebundene } \\
\text { Halb- und Fertigfabrikate }\end{array}$ \\
\hline $\begin{array}{l}\text { Umschlaglager } \\
\text { (Durchgangslager) }\end{array}$ & $\begin{array}{l}\text { hohe } \\
\text { Umschlagleistung }\end{array}$ & $\begin{array}{l}\text { transport- } \\
\text { orientiert }\end{array}$ & $\begin{array}{l}\text { Material, Halb- und Fertig- } \\
\text { fabrikate, Handelswaren }\end{array}$ \\
\hline Verteilungslager & & & \\
\hline - Zulieferungslager & $\begin{array}{l}\text { hohe } \\
\text { Konzentrationsleistung }\end{array}$ & $\begin{array}{l}\text { beschaffungs- } \\
\text { orientiert }\end{array}$ & Material, Handelsware \\
\hline - Auslieferungslager & $\begin{array}{l}\text { hohe } \\
\text { Auflösungsleistung }\end{array}$ & $\begin{array}{l}\text { absatz- } \\
\text { orientiert }\end{array}$ & \\
\hline
\end{tabular}

Tab. 3: Unterscheidung von Lagerhausarten nach ihrer Funktion

Ausschlaggebend für die Funktionserfüllung eines Lagerhauses sind funktionsgerechter Standort und funktionsgerechter Betrieb. Die Standortwahl ist abhängig von Faktoren wie Lieferservice, Art des Absatzgebietes, Nachfrageentwicklung, Verkehrsverbindungen, Transport- und Lagerhauskosten sowie von Arbeitskräften. Weiterhin nehmen die im Lagerhaus ablaufenden Lager- und Bewegungsprozesse Einfluss auf den Standort und die im Lagerhaus eingesetzte Technik. Der Lagerhausbetrieb selbst setzt sich aus den innerbetrieblichen Transporten und aus den Aufgaben Wareneingang, Einheitenlager7, Kommissionierlager, Packerei sowie Warenausgang zusammen. Arten von Lagergütern sind Stückgüter, Schüttgüter, Gase

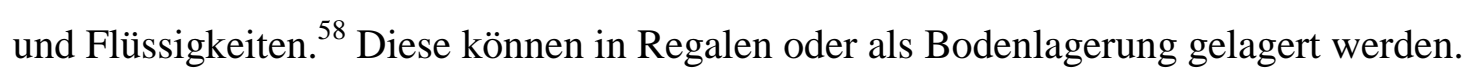

\footnotetext{
Pfohl (Logistiksysteme, 2000) Seite 118.

57 Im Gegensatz zum Wareneingang stehen beim Lagerhausbereich Einheitenlager die Lagerprozesse im Vordergrund. Das Einheitenlager dient ausschließlich der Zeitüberbrückung von Gütern, die in derselben Einheit eingelagert, gelagert und ausgelagert werden. Vgl. Pfohl (Logistiksysteme, 2000) Seite 124. 


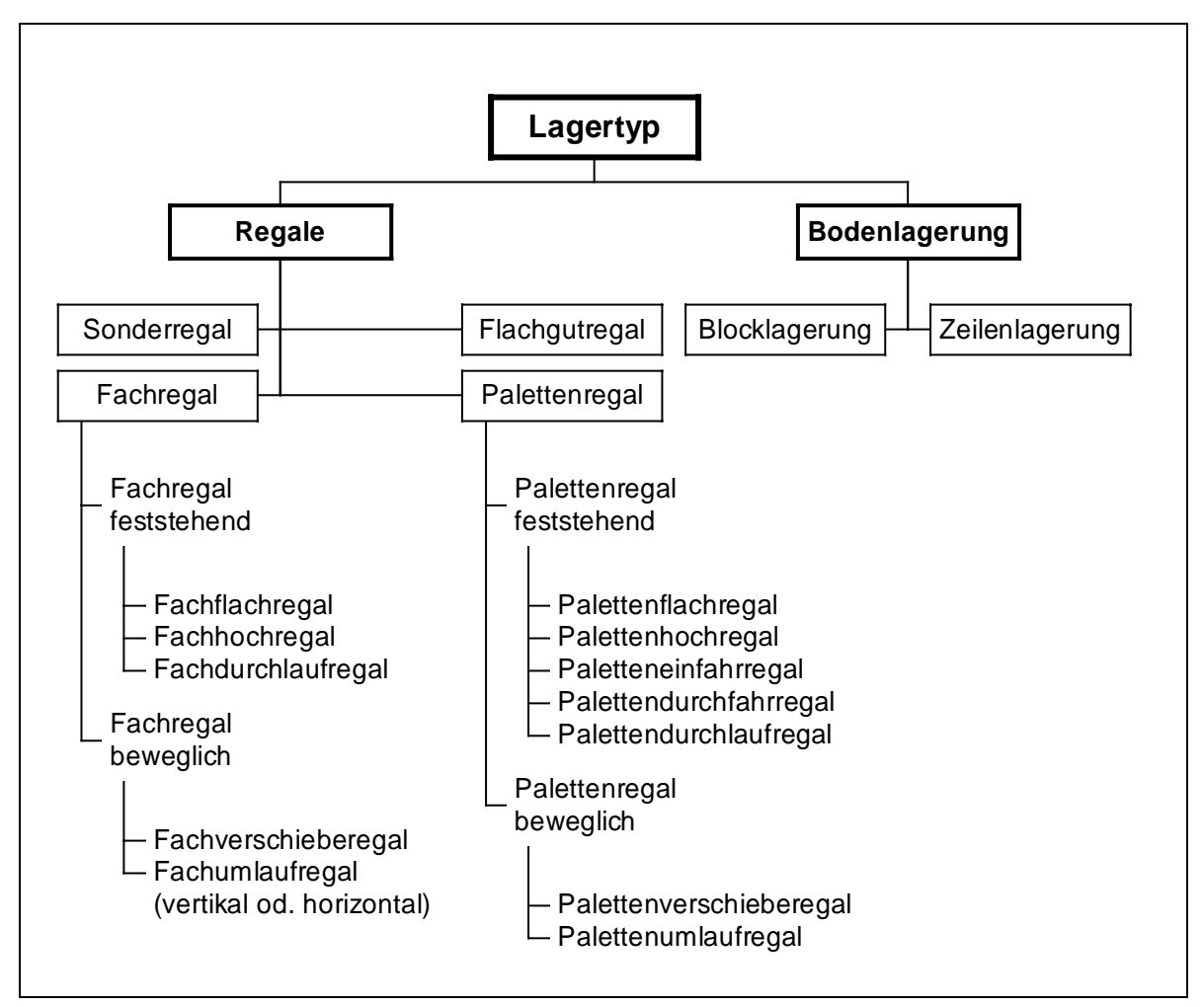

Abb. 4: Lagertypenstruktur

Je nach Art und Type des Lagers sind unterschiedliche Verteil-, Transport- und sonstige technische Systeme notwendig. So benötigt man z.B. für Palettenregale Gabelstapler oder Hebebühnen. Bei größeren schwereren Produkten, die meistens in Block- oder Zeilenlagerung gelagert werden, sind Transportsysteme erforderlich.

All diese Systeme haben einen direkten Einfluss auf die zu erfüllenden Aufgaben und damit auf die Kostenstruktur des Lagers: Fehlen Kapazitäten zum Transportieren, können die nachfolgenden Aufgaben nicht begonnen werden. Sind jedoch zu viele Systeme vorhanden, wirkt sich dies negativ auf die Koststruktur aus.

59 Vgl. Schulte (Logistik, 1995) Seite 91. 


\subsubsection{Die Verpackung in Logistiksystemen}

In der modernen Industriegesellschaft ist die Verpackung zum unverzichtbaren Element einer rationellen und sicheren Warenverteilung geworden: etwa 90\% aller Produkte sind heute einfach oder mehrfach verpackt.

Unter Verpackung versteht man die lösbare Umhüllung eines Gutes (Packgutes), um dieses zu schützen oder andere Funktionen zu erfüllen. Der Verpackungsvorgang wird als Verpackungsprozess bezeichnet. Packgut, Verpackung und Verpackungsprozess bilden zusammen das Verpackungssystem. 61 
Die Eigenschaften bzw. Anforderungen an die Verpackung lassen sich aus den folgenden Funktionen ableiten:

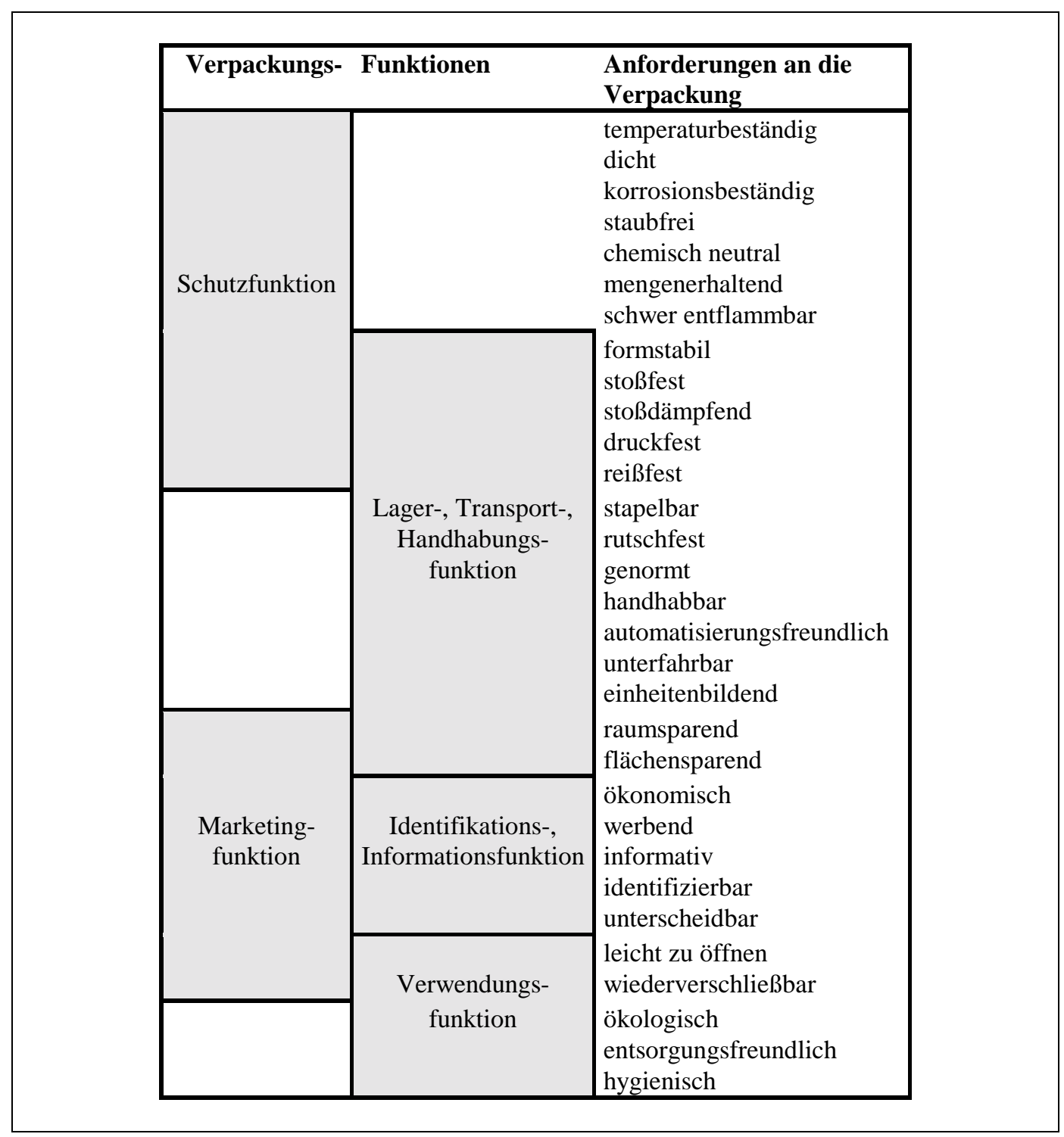

Tab. 4: Grundfunktionen der Verpackung

Die Marketingfunktion einer Verpackung gewinnt verstärkt auch in der klassischen Industrie an Bedeutung. Bislang kannte man „schöne“ bzw. Verpackungen aus hochwertigen Materialien insbesondere bei Süßwaren, Kosmetik, Geschenkartikel oder im Haushaltswarenbereich vorfinden. Heute werden auch Autozubehörteile, Sanitärinstallationssysteme, Computerzubehör oder kleinere Industriemaschinen im Stil

62 Eversheim / Schuh (Betriebshütte, 1996) Seite 16-53 bzw. Jünemann (Materialfluss, 1989) 
des gesamten Firmenauftritts verpackt (Corporate Design), um damit einen weiteren Beitrag zur Corporate Identity zu leisten.

Ziel der Logistikfunktion Verpackung bzw. der „Bildung logistischer Einheiten“ ist die Zusammenfassung von auszuliefernden Gütern zu größeren Einheiten. Der Grundgedanke ist dabei, dass sich der Güterstrom vom Lieferanten zum externen oder internen Kunden bei einem gegebenen Volumen umso reibungsloser gestalten lässt, aus je weniger Bestandteilen er sich zusammensetzt.

Logistische Einheiten sind wiederum Voraussetzung für eine rationelle Transportkette.

Dabei gilt es die folgenden Grundsätze zu beachten:

1. Zusammenfassung der Güter zu größeren Einheiten,

2. Standardisierung der Einheiten in Form und Abmessungen,

3. Erleichterung des Einsatzes mechanischer Mittel bei den Manipulationsvorgängen,

4. Stapelfähigkeit der Einheiten und

5. Wahl der Einheit, die eine weitgehend ununterbrochene Transportkette vom Lieferanten zum Kunden ermöglicht.

Aus der folgenden Abbildung gehen die möglichen Einflussgrößen auf Verpackungsentscheidungen hervor.

Pfohl (Logistiksysteme, 2000) Seite 149.

64 Im Planspiel äußert sich dies im Einsatz von Paletten. 


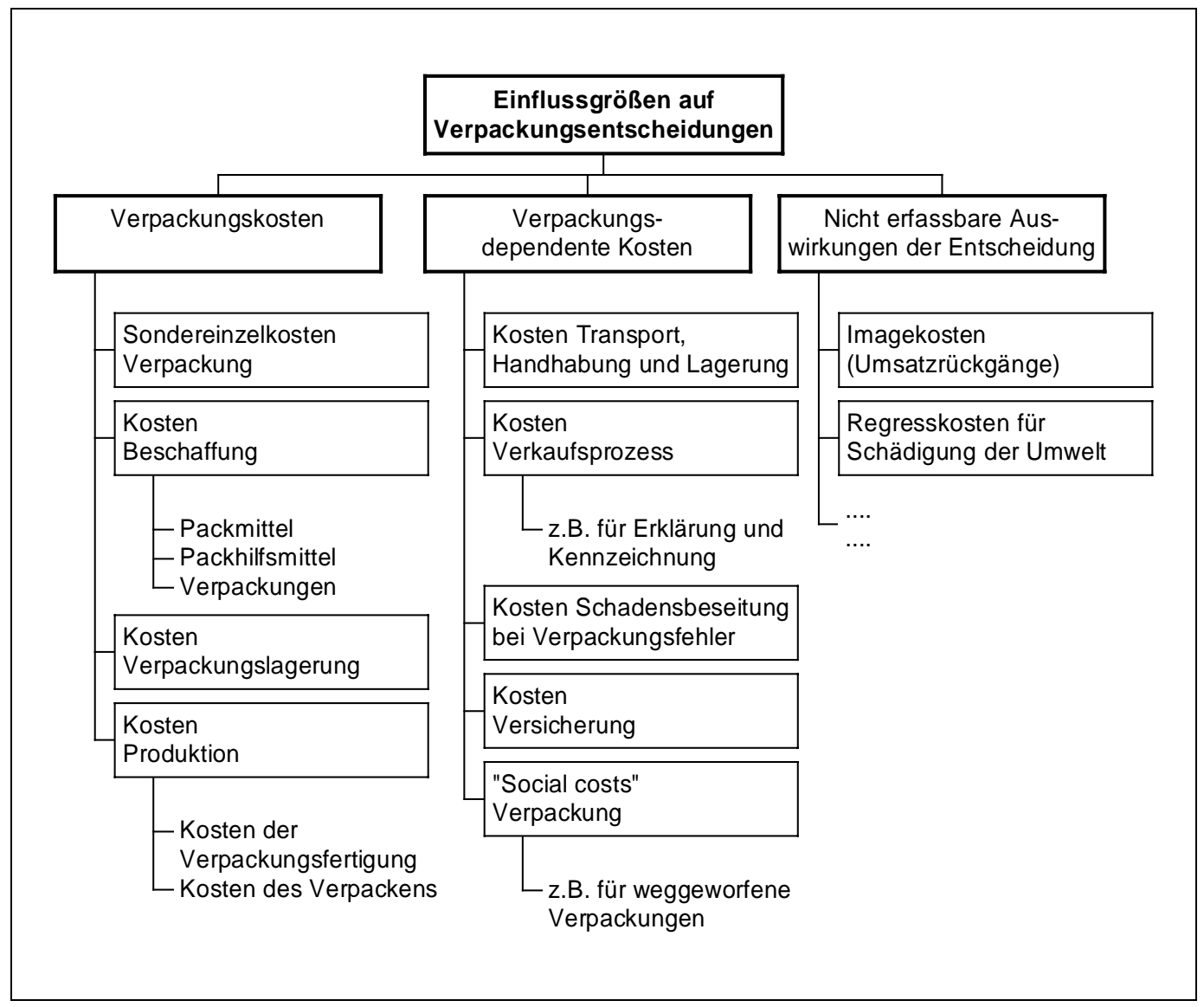

Abb. 5: Einflussgrößen auf Verpackungsentscheidungen

Diese Abbildung veranschaulicht den direkten Einfluss der Logistik auf die Einflussgrößen. So sind insbesondere die Kosten der Beschaffung, die Kosten der Lagerung, die Kosten der Produktion sowie die Kosten für Transport, Handhabung und Lagerung Kosten, die aus den Funktionen der Logistik resultieren.

\subsubsection{Transporte als Hauptfunktionen der Logistik}

Unter Transport - dem letzten der genannten Subsysteme - versteht man die Raumüberbrückung oder Ortsveränderung von Transportgütern mit Hilfe von Transportmitteln. Jedes Transportsystem besteht aus Transportgut, Transportmittel und Transportprozess. Soll ein Transportgut vom Lieferpunkt A zum Empfangspunkt B transportiert werden und ist am Lieferpunkt A nicht das gewünschte Transportmittel 
vorhanden, so kann ein Transportprozess notwendig werden, den man als „Leertransport“ bezeichnet. Leertransporte bilden Transportprozesse ohne Transportgut, die notwendige Voraussetzung für den sich anschließenden Transportprozess mit Transportgut sind. Unter innerbetrieblichem Transport versteht man den Transport in einem Werk von einem Produktionsstandort zum anderen oder den Transport in einem Bereich oder zwischen verschiedenen Bereichen eines Lagerhauses. Als außerbetrieblichen Transport bezeichnet man den Transport vom Lieferanten zum Kunden, den Transport zwischen verschiedenen Werken bzw. zwischen verschiedenen Lagerhäusern eines Unternehmens sowie zwischen den Werken und dessen Lagerhäusern.

Beim sogenannten Transportproblem stellt sich die Aufgabe, von einer bestimmten Anzahl von Absendeorten eine bestimmte Menge von Transporteinheiten zu Bedarfsorten mit einem individuellen Bedarf an Transporteinheiten so zu steuern, dass die Gesamtkosten der Transportdurchführung minimiert werden. ${ }^{6}$ Solche Aufgabenstellungen zeigen sich z.B. bei der Belieferung von mehreren Zweigwerken, Lagerhäusern, Auslieferlägern und Großabnehmern durch mehrere (Vor-) Erzeugungswerke, beim Leerwagenumlauf von Eisenbahnen und Transportunternehmungen und bei der Einsatzplanung innerbetrieblicher Fördermittel. Für die Lösung solcher Transportprobleme eignet sich die Lineare Optimierung.

Dazu ein kurzes Beispiel. Es existieren drei Absendeorte $A_{1}$ bis $A_{3}$ (z.B. Produktionsstätten) und vier Bedarfsorte $B_{1}$ bis $B_{4}$ (z.B. Händler), die jeweils bestimmte Kapazitäten auf Lager haben (Angebot) bzw. benötigen (Bedarf). Die Transporte von $A_{\mathrm{i}}$ nach $B_{\mathrm{j}}$ verursachen Kosten $C_{\mathrm{ij}}$ je Transporteinheit, die in der Tabelle wiedergegeben werden.

65 Vgl. Ritter (Verpackungsoptimierung, 1980) Seite 43 ff.

66 Pfohl (Logistiksysteme, 2000).

67 Vgl. hier und im nachfolgenden Kern (Operations Research, 1966) Seite 39 ff. Weitere Beispiele findet man bei Arlt (Netzwerkflussprobleme, 1994) Seite 20 ff., Meyer / Hansen (Planungsverfahren, 1996) Seite 5 ff. oder Pack (Planspiele, 1968) Seite $17 \mathrm{ff}$.

68 Das hier beschriebene problem ist ausgeglichen, d.h. Summe Angebot = Summe Nachfrage. 


\begin{tabular}{|c|c|c|c|c|c|}
\hline & $\mathbf{B}_{\mathbf{1}}$ & $\mathbf{B}_{\mathbf{2}}$ & $\mathbf{B}_{\mathbf{3}}$ & $\mathbf{B}_{\mathbf{4}}$ & Angebot \\
\hline $\mathbf{A}_{\mathbf{1}}$ & 90 & 150 & 40 & 80 & 9 \\
\hline $\mathbf{A}_{\mathbf{2}}$ & 30 & 80 & 70 & 10 & 10 \\
\hline $\mathbf{A}_{\mathbf{3}}$ & 20 & 40 & 20 & 60 & 3 \\
\hline Bedarf & 5 & 6 & 7 & 4 & 22 \\
\hline
\end{tabular}

Tab. 5: Kosten-, Angebots- und Bedarfsmatrix eines Transportproblems

Eine erste Lösung findet sich mit der sogenannten Nordwest-Ecken-Regel. Sie besagt, dass man für die obere linke Ecke der Tabelle soviel Einheiten einplant, dass der Bedarf von $B_{1}$ (5 Einheiten) möglichst erfüllt, die Überschussmenge von $A_{1}$ (9 Einheiten) jedoch nicht unterschritten wird. Man schreitet solange zum nächsten waagrechten Feld, bis keine Überschussmenge mehr vorhanden ist. Im Anschluss daran wechselt man senkrecht in der gleichen Spalte zur nächsten Zeile und plant dort abermals die erforderliche bzw. höchstmögliche Menge. In obigem Beispiel ergibt sich folgende Verteilung:

\begin{tabular}{|c|c|c|c|c|c|}
\hline & $\mathbf{B}_{\mathbf{1}}$ & $\mathbf{B}_{\mathbf{2}}$ & $\mathbf{B}_{\mathbf{3}}$ & $\mathbf{B}_{\mathbf{4}}$ & Angebot \\
\hline $\mathbf{A}_{\mathbf{1}}$ & 5 & 4 & & & 9 \\
\hline $\mathbf{A}_{\mathbf{2}}$ & & 2 & 7 & 1 & 10 \\
\hline $\mathbf{A}_{\mathbf{3}}$ & & & & 3 & 3 \\
\hline Bedarf & 5 & 6 & 7 & 4 & 22 \\
\hline
\end{tabular}

Tab. 6: Erste Lösung des Transportproblems

Die Gesamtkosten $K$ errechnen sich aus der Summe der Einzelkosten $C_{\text {ij }}$ multipliziert mit den vorgesehenen Transportmengen $X_{\mathrm{ij}}$. Sie betragen für die erste Lösung $K=\underset{i, j}{ } C_{i j} * X_{i j}=1.890 €$

Diese erste Lösung ist jedoch nicht die optimale Lösung. Mittels verschiedener Verfahren und Methoden ${ }^{69}$ lassen sich weitere und häufig deutlich bessere Lösungen finden. Diese werden teilweise auch die nicht verwendeten Feldkombinationen bewerten. So wird z.B. bei der ersten Lösung auf der billigsten Transportstrecke von $A_{2}$

69 Außer der Nordwest-Ecken-Methode gibt es das Zeilen-Spalten-Sukzessionsverfahren, das Zeilenfolge- oder Spaltenfolgeverfahren, das Matrixminimumverfahren, das Verfahren des doppelten Vorzuges, die Frequenzmethode, die Vogel'sche Approximation und das Verfahren von Russel. Diese bieten Lösungen an, worunter sich die optimale Lösung befinden kann. Vgl. dazu Bloech (Optimalplanung, 1999) Seite 61 ff. oder Bloech et al. (Konzernlogistik, 1994) 
nach $B_{4}$ (Kosten $10 €$ ) nur 1 Transportmenge transportiert. Bei der optimalen, nachfolgend dargestellten Lösung wird jedoch in diesem speziellen Beispiel das mögliche Maximum von 4 Einheiten transportiert.

\begin{tabular}{|c|c|c|c|c|c|}
\hline & $\mathbf{B}_{\mathbf{1}}$ & $\mathbf{B}_{\mathbf{2}}$ & $\mathbf{B}_{\mathbf{3}}$ & $\mathbf{B}_{\mathbf{4}}$ & Angebot \\
\hline $\mathbf{A}_{\mathbf{1}}$ & 2 & & 7 & & 9 \\
\hline $\mathbf{A}_{\mathbf{2}}$ & 3 & 3 & & 4 & 10 \\
\hline $\mathbf{A}_{\mathbf{3}}$ & & 3 & & & 3 \\
\hline Bedarf & 5 & 6 & 7 & 4 & 22 \\
\hline
\end{tabular}

Tab. 7: Optimale Lösung des Transportproblems

Die optimale Lösung verursacht Kosten in Höhe von $950 €$ und ist damit um die Hälfte billiger als die erste Lösung.

\subsection{Kenngrößen der Logistik}

Logistikleitungen sind als Dienstleistungen generell schlechter erfassbar als Sachleistungen, die man zählen, messen oder wiegen kann. 00 Dies gilt insbesondere deswegen, weil einzelne Logistikbegriffe nur schwer abgrenzbar sind.

Um die Logistikleistung zu bewerten ist die ständige Überprüfung verschiedenster Zielgrößen ${ }^{\text {nötig und hilfreich: }}$

Die Lieferbereitschaft oder auch Liefertreue als zentrale Größe zur Bewertung des Logistiksystems werden durch den Lieferbereitschaftsgrad oder auch Servicegrad beschrieben. Er berechnet sich aus dem Wert der pünktlich ausgelieferten Aufträge durch den gesamten Bestellwert der Aufträge. Die Kennzahl gibt an, welcher wert- oder mengenmäßige Anteil der ausgelieferten Aufträge termingerecht geliefert wurde. Termingerecht ist ein Erzeugnis dann, wenn das jeweilige Produkt vollständig zum

\footnotetext{
Ewersheim / Schuh (Betriebshütte, 1996) Seite 18-22.

Vgl. Strauß (Controlling, 1993) Seite 190.

72 Vgl. hier und im folgenden: Ewersheim / Busch (Betriebshütte, 1996) Seite 15-80 ff. und Strauß (Controlling, 1993) Seite 193.
} 
zugesagten Termin ausgeliefert wurde oder innerhalb der Zeitspanne, die vom Kunden als zeitlicher Verzug akzeptiert wurde. Eine weitere wichtige Kennzahl ist der Lagerauslastungsgrad. Er berechnet sich als durchschnittlich belegt Lagerfläche durch verfügbare Lagerfläche in $\mathrm{m}^{2}$. Der Auslastungsgrad ist ein Maß für die Kapazitätsauslastung der Lager und eignet sich für Wirtschaftlichkeitsanalysen im Lagerbereich. Der absolute Wert für die Lagerbestandshöhe kann relativiert werden, indem das Verhältnis zum Umsatz gebildet wird. Die entsprechende Kennzahl ist der Anteil der Bestände am Umsatz. Diese Kennzahl beschreibt den wertmäßigen Anteil der Bestände am Umsatz. Damit wird eine Aussage über die Bevorratungsintensität geliefert, die zur Messung und Analyse von Höhe und Zusammensetzung der Lagerbestände eingesetzt wird. Lieferzeit ist die Zeitspanne vom Datum der Auftragserteilung bis zum Auftragserfüllungstermin. Lieferfähigkeit ist der Grad der Übereinstimmung zwischen Wunschtermin und zugesagtem / bestätigtem Auftragserfüllungstermin. Lieferqualität oder auch Lieferzuverlässigkeit ist der Anteil der ausgeführten Aufträge ohne qualitative und quantitative Mängel. Die Kennzahl kann dabei getrennt auf Auftragsprodukte, Lagerprodukte oder Ersatzteile bezogen werden. Flexibilität ist die Fähigkeit, auf Kundenwunschänderungen hinsichtlich Spezifikationen, Mengen und Terminen einzugehen. Dazu gehören Abnahmemengen, Zeitpunkt der Auftragserteilung, Art der Auftragsübermittlung, Art der Verpackung, die alternativen Transportvarianten oder die Möglichkeit zur Lieferung auf Abruf. Sie wird gemessen in Anzahl der erfüllten Sonderwünsche durch die Anzahl der Sonderwünsche. Problematisch ist hierbei die genaue Abgrenzung und insbesondere die Erfassung. Informationsbereitschaft ist die Fähigkeit, in allen Stadien der Geschäftsabwicklung vorgangs- und produktbezogen auskunftsbereit zu sein.

Mit zunehmender Bedeutung der Logistik für den Unternehmenserfolg nehmen auch die damit verbundenen Kosten zu. Der Anteil der Logistikkosten am Umsatz ist deshalb eine wichtige Messgröße. Diese Kennzahl stellt die Kosten der Logistik dem am Umsatz gemessenen Unternehmenserfolg gegenüber. Die Logistikkosten ergeben sich aus den auf den relevanten Kostenstellen anfallenden Kostenarten. Relevante Kostenstellen sind die Bereiche Fertigfabrikatlager, Kommissionierung, Verpackung, Versand, außerbetrieblicher Transport und Auftragsabwicklung. Kostenarten sind Personalkosten, 
Betriebsmittelkosten, Raumkosten, Abschreibungen, Zinsen auf Einrichtungen und Lagerbestände, Steuern, Gebühren und Versicherungen.

Weitere Möglichkeiten zur Erfassung und Transparenz der Logistikosten bieten die folgenden Kennzahlen: Lenkungskosten sind Kosten für das Steuern und Abwickeln der Aufträge und Bestellungen sowie für die Planung und Bereitstellung der Einsatzfaktoren. Transport-/ Handlingskosten sind Kosten für die Durchführung des Material/Warenflusses. Lagerkosten sind Kosten für die Einlagerung, Lagerung und Auslagerung von Material und Waren (Vorräte). Kapitalbindungs- und Wagniskosten sind Kosten für in der gesamte Supply Chain 74 gebundenen Materialien und Waren (Vorräte).

Zusätzlich werden speziell auf die distributionslogistischen Teilbereiche bezogene Kennzahlen und absolute Messgrößen in der betrieblichen Praxis eingesetzt. Dazu gehören z.B. Anzahl der Bestellungen pro Monat, Anzahl der Positionen pro Auftrag, durchschnittliche Wert je Bestellung, durchschnittliche Kosten pro Bestellung, Lagerhaltungskostensatz, Lagerkapazität, durchschnittliche Anzahl der Kommissionieraufträge je Kommissionierer, durchschnittliche Anzahl der Positionen je Kommissionierauftrag, durchschnittliche Kommissionierzeit je Auftrag, durchschnittliche Kosten pro Kommissionierauftrag, Transportkosten je Tonnenkilometer, Transportkosten je Sendung und Anteil der Personalkosten an den Logistikosten.

\subsection{Aktuelle Entwicklungen und Probleme}

Rationalisierungsmöglichkeiten in den Fertigungsprozessen sind oft ausgeschöpft. Gute Chancen zur Erschließung von Kostenreserven bieten sich aber innerhalb der

73 Beim Einsatz der Kennzahl für den zwischenbetrieblichen Vergleich müssen die betrachteten Kosten und die bei der Errechnung eingesetzten Kostenrechnungssysteme genau analysiert werden, um Fehlinterpretationen zu vermeiden. Vgl. Ewersheim / Busch (Betriebshütte, 1996) Seite 15-81.

74 Unter der Supply Chain versteht man die Verbindungskette zwischen den Herstellern und ihren Zulieferern, den als Absatzmittlern fungierenden Groß- und Einzelhändlern, den zu den Absatzhelfern zählenden Logistikunternehmen und de Endabnehmern. Vgl. Pfohl (Logistiksysteme, 2000) Seite 327.

75 Ewersheim / Busch (Betriebshütte, 1996) Seite 15-81 f. 
Logistikkette. ${ }^{76}$ Dies gilt einerseits für den innerbetrieblichen Materialfluss und für die Vorgänge und Abläufe innerhalb der Läger, andererseits für internationale Wertschöpfungsketten.

Ein wichtiger Hebel ist in diesem Zusammenhang die Bestandsführung. Umlaufvermögen stellt bekanntlich gebundenes Kapital dar. ${ }^{77}$ Eine Vergrößerung des Umlaufvermögens wird in der Regel von einer Vergrößerung der Verbindlichkeiten begleitet. Die Folgen sind höhere Zinsbelastungen bzw. höhere Kapitalbindungskosten. Bei Unterschreitung der Lagerbestände bzw. völligem Fehlen von Halbfabrikaten oder Rohstoffen sind die Auswirkungen noch viel größer, da einerseits kein Umsatz zustande kommt, andererseits keine Wertschöpfung geschieht. Dass die Mitarbeiter und Maschinen eventuell sogar ,leer“ arbeiten und damit die kalkulierten Stundensätze nicht mehr „stimmen“, kommt erschwerend hinzu.

Wildemann weist darauf hin, dass Umlaufvermögen Fehler verdeckt: Die Optimierung einzelner Funktionen wie Einkauf, Produktion und Vertrieb erfordert Umlaufvermögen, das eine reibungslose Produktion, prompte Lieferung, Überbrückung von Störungen, wirtschaftliche Fertigung und eine konstante Auslastung der Produktionsanlagen erst ermöglicht. In einer Produktion, die auf ein „Fließen“ ausgerichtet ist, verdecken Bestände störanfällige Prozesse, unabgestimmte Kapazitäten, mangelnde Flexibilität, Ausschuss und mangelnde Liefertreue. Senkt man die Bestände, werden diese Probleme offensichtlich und es entsteht ein unmittelbarer Zwang, diese zu lösen. Hierdurch wird eine permanente Produktivitätssteigerung des Produktionsgeschehens initiiert. 08

Auch die Warenverteilung innerhalb eines Produktionsverbundes und die damit verbundenen Handhabungsvorgänge wurden in den vergangenen Jahren für viele Unternehmen zu einem kritischen Erfolgs- und Kostenfaktor. Entsprechend groß ist das Interesse an Automatisierungslösungen zur Reduzierung der Distributionskosten, Verkürzung der Durchlauf- und Lagerzeiten, Steigerung der Qualität im

\footnotetext{
Busch (Logistikkette, 1998).

Vgl. Wildemann (Logistik, 1992) Seite 193.

Wildemann (Logistik, 1992) Seite $193 \mathrm{f}$.
} 
Warenumschlag, kurz: zur effizienten Beherrschung der komplexer werdenden Vorgänge im Bereich der Warenlogistik.

Weiterhin ist die Transparenz der Auftragsabwicklung ein wachsender Wettbewerbsfaktor. ${ }^{80}$ Dazu ist es erforderlich, Standort und Status von Sendungen, Fahrzeugen, Transportgefäßen und Ladehilfsmitteln in der logistischen Kette zu identifizieren. Elektronische Informationsträger, die austauschbar oder fest mit dem Objekt verbunden sind, gewinnen wegen des mannigfaltigen Zusatznutzens an Verbreitung. Für die Informationsübertragung kommen in jüngerer Zeit auch Datenfunksysteme zum Einsatz. 81

Der Verdrängungswettbewerb innerhalb der Speditionsbranche - bedingt durch zunehmende Transparenz der eigentlichen Transportleistung, unabhängig vom Land-, See- oder Luftfrachtverkehr - erfordert ein Höchstmaß an Servicequalität, was zwangsläufig zu neuen Anforderungen an die Kommunikationsprozesse mit ihren informationstechnischen Systemen führt. Nur durch gezielte Differenzierung vom Wettbewerb lassen sich neue Kundenpotenziale erschließen bzw. bestehende Kundenverhältnisse stabilisieren. 82

In der Speditionsbranche entwickeln sich die ehemaligen reinen Transportunternehmen vermehrt zu integrierten Logistikunternehmen mit geschlossener Logistikkette. Dabei zeichnet sich die Tendenz zur papierlosen Spedition ab. $\stackrel{83}{ }$ Papierlos bedeutet in diesem Zusammenhang, dass alle Informationen $\mathrm{zu}$ einem Auftrag ausschließlich über Computersysteme abrufbar sind. Es ist nicht nötig und auch nicht gewünscht, einen Ausdruck auf Papier zu erstellen, da dieser Ausdruck veraltet ist, wenn sich eine Information (nachträglich) ändert.

79 Severin (Warenumschlag, 1998).

8060 Prozent aller Brillenglasaufträge werden erst nach Vorliegen des Kundenauftrages individuell berechnet und gefertigt. Dennoch müssen Lieferzeiten für diese Aufträge von maximal zwei bis drei Arbeitstagen erreicht werden. Auftragsannahme, Produktion und Distribution sind europaweit in zwei bis drei Tagen, Lageraufträge über Nacht sicherzustellen. Vgl. Femppel (Kundenlogistik, 1998).

81 Lucke (Information, 1998).

82 Otto et al. (Nadelöhr, 1998). 
Durch die vielfältigen Aufgaben der Logistik und die zunehmende Konzentration in der Industrie und beim Handel auf core business und Globalisierung steigt die Bedeutung von Dienstleistern in diesem Bereich stetig. Netzwerke und Kooperationen verringern zwar die Komplexität der Abläufe im einzelnen Unternehmen, bringen jedoch erhöhten Koordinationsaufwand im Gesamtsystem mit sich. Es nimmt das Bedürfnis nach einem neutralen Koordinator zu, der den aus einer Vielzahl von Einzelprozessen bestehenden Gesamtprozess steuert. Da dies wiederum nicht zur Kernkompetenz der beteiligten Unternehmen zählt, werden Steuerung und Lenkung der geschilderten Prozesse sinnvollerweise auf einen externen (Logistik-) Dienstleister übertragen. 84

Als Folge existiert ein stark wachsender Markt auf dem Gebiet der sogenannten Kontraktlogistik. Dabei übernimmt ein Dienstleistungsunternehmen z.B. Personal, Logistikzentren und Fuhrpark des beauftragenden Unternehmens. ${ }^{85}$ Hier liefern sich namhafte Unternehmen wie Danzas, Panalpine oder Kühne \& Nagel einen intensiven Wettbewerb.

Allerdings kann speziell die Abweichung von Planzahlen zu gravierenden Problemen führen, wie das folgende Praxisbeispiel verdeutlicht.

Für den Opel Zafira liegen in Europa mehr als 300.000 Bestellungen vor. Wie das amerikanische Fachblatt „Automotive News“ berichtet, will man die große Nachfrage wenigstens zum Teil damit auffangen, dass Fahrzeuge aus Thailand nach Europa geschickt werden. Neben Bochum ist Rayong der einzige Produktionsstandort des Minivan. Dort sollen jetzt statt 40.000 Autos jährlich 60.000 produziert werden. Dafür wird die Zahl der Mitarbeiter von 650 auf 1.200 erhöht. 15.000 der 20.000 zusätzliche Zafiras sollen also nach Europa geliefert werden. Zur Zeit beliefert Rayong außer dem thailändischen Markt nur Chile, Singapur und die Philippinen. Nach Indonesien werden im September

83 FAZ (28. August 2000): Das Familienunternehmen Hellman Worldwide Logistics GmbH \& Co. KG setzte 1999 ca. 1,8 Mrd. € um, wobei der Auslandsanteil über 55 Prozent betrug. Ein großer Teil der Investitionen von knapp 40 Mio. $€$ floss in Datenverarbeitung und Software.

84 Fiege (Dienstleister, 1998). In einer Untersuchung der Automobilhersteller zeigt sich, dass als vorrangige Aufgaben insbesondere die tägliche Anlieferung im Rahmen von Just-in-Time-Konzepten, die Integration von Qualitätsmanagementsystemen des Wertschöpfungsnetzwerkes, die Logistikberatung, Verpackungs- und Kommissionieraufgaben und das Lagerhausmanagement gesehen werden. Vgl. Göpfert et al. (Beschaffungslogistik, 1999) Seite 242 ff.

85 FAZ (30. August 2000): Die Danzas Holding AG, Tochter der Deutschen Post AG, wird von der Deutschen Telekom AG die gesamte Lagerung und Verteilung von Material übernehmen. Dieser Umsatzzuwachs von 200 Mio. $€$ entspricht ca. 5 Prozent des derzeitigen Nettoumsatzes von Danzas. 
auch Großbritannien, Frankreich, Spanien und Italien Exportländer für den thailändischen Zafira.

Die gesamte Logistik besitzt für industrielle aber auch für New Economy-Unternehmen eine beachtliche Bedeutung. In der produzierenden Industrie begründet dies insbesondere der Wertanteil, den das Material an Umsatzerlösen und Vorräten aufweist. Im Bereich der New Economy dominieren weniger die Wertanteile als fristgerechte Lieferungen. Was immer im e-commerce bestellt und gehandelt wird, muss zumeist auch physisch geliefert werden.

\subsection{Einschlägige Berufe und Aufgaben}

Zur Abrundung der Logistikpraxis sollen einige Berufsbezeichnungen sowie dafür notwendige Qualifikationen aus dem Bereich der Logistik Erwähnung finden.

Einkäufer sollen nicht nur günstig, sondern optimal einkaufen. Bei der Beschaffung der nötigen Produktionsmittel und -anlagen müssen sie neben den Kosten insbesondere Termintreue und Qualitätsgesichtspunkte im Blick behalten. Bei der Berechnung der optimalen Bestellmengen spielen auch Lagerhaltungs- und Kapitalbindungskosten eine wesentliche Rolle. Die Aufgaben im Einkauf wachsen insgesamt zunehmend in Richtung Logistik und integrierter Materialwirtschaft.

Der früher von Praktikern besetzte Bereich wird zunehmend von Akademikern eingenommen. Sein einst blasses Image hat der Einkauf längst abgelegt. Wegen des zunehmenden internationalen Wettbewerbs und der Verkürzung der Produktlebens- und Innovationszyklen wächst mit den Kostensenkungspotenzialen die strategische Bedeutung des Beschaffungsbereichs.

86 FAZ (19. August 2000 A) Seite 58.

87 FAZ (9. Oktober 2000) Seite 35. Ein Beispiel für die virtuelle Wirtschaft ist die Firma „Amazon.com“. Auf einer virtuellen Plattform können Bücher, CDs, Arzneimittel oder ähnliche klar definierte Produkte bestellt werden. Diese sind innerhalb weniger Tage - so die Unternehmung - kostenfrei beim Besteller. Auf diesen Bereich setzt auch die Deutsche Post AG bei ihrem Börsengang im Herbst 2000.

88 Vgl. dazu umfassend Achtenhagen (Lernhandeln, 1992), Achtenhagen (Schlüsselqualifikationen, 1997) oder Staufenbiel (Berufsplanung, 2000). 
$\mathrm{Zu}$ den Anforderungen gehören Kenntnisse über Beschaffungslehre und -marketing, Produktionswirtschaft/Marketing, Wertanalyse/Finanzierung, Lagerhaltungsmodelle, Logistik, Datenverarbeitung, ABC-Analyse sowie juristische Kenntnisse (z.B. Produkthaftungsrecht).

Der zentrale Einkäufer (oder auch strategische Einkäufer) koordiniert (meistens verschiedene) Geschäftseinheiten in deren Bestellwesen. Seine Aufgabe ist es, durch Skaleneffekte Synergien im Bestellbereich zu finden. Beschaffungsmarktforschung, Beschaffungsanbahnung und -abschluss, Preis- und Wertanalysen sowie Beschaffungsverwaltung sind seine typischen Aufgaben.

Der Logistikcontroller ${ }^{91}$ ist Gestalter des Logistikinformationsmanagements, Mitwirkender in der Logistikplanung und Durchführender der Logistikkontrolle. Logistikcontrolling hat zum Ziel eine permanente Wirtschaftlichkeitskontrolle durch Soll-Ist-Vergleiche von Kosten und Leistungen sowie die Beschaffung, Verdichtung und Bereitstellung entscheidungsbezogener Informationen. Controlling bedeutet dabei nicht nur Kontrollieren, sondern vor allem auch Beherrschen, Regeln und Steuern. ${ }^{22}$ Der Logistikcontroller entwickelt u.a. Logistikinformationssysteme, koordiniert den Informationsbedarf und die Informationsverwendung in der Logistik (Vermeidung von Zahlenfriedhöfen) und gewährleistet damit ein einheitliches, formalisiertes System der Logistikplanung. Weiterhin erarbeitet er logistikpolitische Ziele, ermittelt Ist-Größen und stellt Zielereichungsgrade durch unternehmensinterne oder -externe Vergleiche fest.

Logistikexperten - oder auch Materialwirtschaftler - optimieren die Materialflüsse zwischen den Herstellern und Kunden im Hinblick auf Durchlaufzeiten, Kosten und Qualität. Die immer stärker differenzierten Warenströme und Produktionsprozesse müssen zu einem sinnvollen Ganzen zusammengefügt werden. Logistiker stellen mit

89 Staufenbiel (Berufsplanung, 2000) Seite 180. Zum Begriff des Einkaufs-Controlling vgl. umfassen Katzmarzyk (Einkaufscontrolling, 1988).

$90 \quad$ Schulte (Logistik, 1995) Seite 127.

91 Schulte (Logistik, 1995) Seite 355.

92 Strauß (Controlling, 1993) Seite 183. 
Materialflusskonzepten wie dem Just-in-time- oder KANBAN-Verfahren $\stackrel{03}{\text { die }}$ Fertigungs- und Lieferbereitschaft sicher. Bei der Analyse der existierenden Materialflüsse spielen neben optimalen Losgrößen, Kosten-, Termin- und Qualitätsgesichtspunkten auch ökologische Aspekte eine Rolle. Datenverarbeitungssysteme kommen in Form von automatisierten Materialflusssteuerungen und bei der integrierten Materialwirtschaft zum Einsatz. Logistiker müssen innerbetriebliche Erfordernisse - etwa in Bezug auf die Materialbeschaffung, Produktion, Zwischenlagerung, Kommissionierung oder den Weitertransport - mit den außerbetrieblichen Bedingungen der Lieferanten und Kunden abstimmen. Auch Outsourcing kann sich bei der Distribution als kostengünstigste Lösung herausstellen, da hier insbesondere die Fixkosten entfallen. Beim Logistikmanagement nimmt unternehmensübergreifende Teamarbeit durch moderne Informations- und Kommunikationssysteme zu. Neue Herausforderungen an die Logistiker stellen insbesondere elektronische Handelssysteme (e-commerce). Wichtigste Anforderungen an den Logistikexperten sind Kenntnisse aus den Bereichen Industriebetriebslehre/ Produktionswirtschaft, Materialwirtschaft, Warenwirtschaft, Logistik, Marketing, Kostenrechnung, Verkehrspolitik, OR-Methoden und Recycling. 04

Durch die Analyse der Kosten und Erträge in den simulierten Unternehmungen lassen sich Entscheidungen konsequent vorbereiten und fundieren. Optimierungs- oder Näherungslösungen werden leichter erfassbar als in einer isolierten Situation. Das Erfolgsverständnis der Logistikmanager kann insoweit vertieft werden. Das Herunterbrechen der Unternehmungsziele auf die Ziele der Logistikbereiche bis hin zu den ausführenden Aktivitäten führt zu den logistischen Handlungsweisen. Die Frage ist, welchen komplexen Entscheidungssituationen begegnen Logistikmanager ?

93 Das japanische KANBAN-System (Kanban, japanisch „Schild“ oder „Karte“) ist ein Ansatz, die Produktion transparenter zu gestalten, Abläufe zu entwirren und damit beherrschbar zu machen. Die wichtigsten Elemente sind (a) Gliederung der Produktion in ein System vermaschter, sich selbst steuernder Regelkreise, bestehend aus jeweils einem teileverbrauchenden Bereich (Senke) und einem dazugehörigen vorgelagerten teileerzeugenden Bereich (Quelle), (b) Aufbau eines Zwischenlagers (Puffers) zwischen Quelle und Senke, um Unregelmäßigkeiten oder Störungen im Produktionsablauf auszugleichen, (c) Einführung eines Holprinzips (Pull-Prinzip) für den jeweils nachfolgenden, verbrauchenden Bereich, (d) Nutzung spezieller Informationsträger, die als Kanban-Karten zur eigentlichen Fertigungssteuerung dienen und (e) Übertragung der kurzfristigen Steuerungsverantwortung an die ausführenden Mitarbeiter, so dass keine zentrale Fertigungssteuerung mehr erforderlich ist. Vgl. Eversheim / Schuh (Betriebshütte,1996) Seite 14-84 ff.

94 Staufenbiel (Berufsplanung, 2000) Seite 182. 
Als Beispiele für komplexe Entscheidungssituationen seien genannt:

1. Transportprobleme in verzweigten Verkehrssystemen mit Umladepunkten und mehreren Quellen und Bedarfsorten.

2. Lagerhausprobleme mit gleichzeitiger Transport- und Lagerdisposition.

3. Versorgungssysteme im Produktions- und Distributionsbereich der Unternehmung.

4. Benchmark-Auswertungen in der Transportwirtschaft und Lagerhaltung.

5. Logistiksysteme für eine international verteilte Produktion.

6. Outsourcing-Planungen für Logistikzentren.

Untersucht man diese Beispiele, stellt man fest, dass vom Logistiker insbesondere Kompetenz für Optimierungsfragen gefordert wird. Diesem Umstand sucht das Wissenschaftsgebiet Operations Research ${ }^{60}$ gerecht zu werden.

Zusammenfassend kann man die Aufgaben eines (Logistik-) Managers wie folgt beschreiben: Erkennen von Strukturen, Abläufen, Störungen, Problemen und der Wirksamkeit von Problemlösungen.

95 Rundereiseproblem, auch Travelling Salesman- oder Chinese Postman-Problem genannt. Vgl. z.B. Bloech / Ihde (Distributionsplanung, 1972) Seite $109 \mathrm{f}$.

96 Operations Research bedeutet, bestimmte komplexe Problemstellungen mit Hilfe von mathematischnaturwissenschaftlichen Methoden einer Lösung zuzuführen. Es handelt sich dabei um Problemstellungen, wie sie insbesondere in privates und öffentlichen Unternehmen auftreten, wenn dort Systeme, bestehend aus Menschen, Maschinen, Material und Kapital, zu planen und zu leiten sind. Charakteristisch für den Lösungsversuch ist die Abbildung der Systeme durch mathematische Modelle, die es zulassen, alternative Entscheidungen, Strategien oder Maßnahmen hinsichtlich ihres Zielerreichungsgrades zu vergleichen. Dabei sind Entscheidungen wie Ungewissheit und Trends zu berücksichtigen. Der Zweck von Operations Research besteht darin, diejenigen zu unterstützen, die Systeme der genannten Art gestalten und leiten. Vgl. Beer (Cybernetics, 1959) bzw. Meyer (Systemforschung, 1995) Seite 1. 


\section{Planspiele und Unternehmenssimulationen für Konkurrenzsituationen}

Planspiele vermitteln Führungskräften aus dem Bereich der Wirtschaft neben einem Technical Skill und Social Skill auch einen Conceptual Skill, nämlich ,die Fähigkeit zur ,Zusammenschau“ der komplexen und interdependenten Kräfte, die innerhalb und außerhalb eines Unternehmens wirksam sind und seine Leitung zum Problem werden lassen, sowie zur Entwicklung einer dieser Kräfte berücksichtigenden Führungskonzeption.“ 97

Ludwig Pack

\subsection{Merkmale von Planspielen}

Simulation ist die Nachbildung eines dynamischen Prozesses in einem Modell, um zu Erkenntnissen zu gelangen, die auf die Wirklichkeit übertragbar sind.

\subsubsection{Historische Entwicklung}

Unmittelbare Vorbilder der heute bekannten wirtschaftlichen Planspiele sind

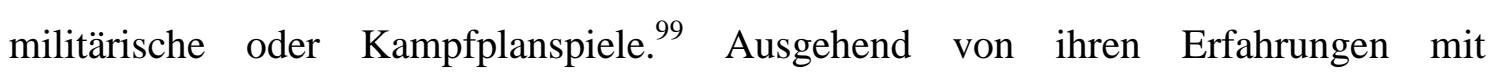
Kriegsspielen, schuf die RAND Corporation in Santa Monica (USA) im Jahre 1955 das Planspiel „Monopologs“, das sich überwiegend mit betriebswirtschaftlichorganisatorischen Fragen des Nachschubwesens der amerikanischen Luftwaffe befasst

97 Vgl. Pack (Planspiele, 1968) Seite 7.

98 Vgl. Verein Deutscher Ingenieure (VDI-Richtlinie 3633, 1996). „Simulation“, von lateinischer Herkunft, bedeutet in Wissenschaft und Technik die modellhafte Nachbildung eines beliebigen Systems oder Prozesses durch ein anderes (kybernetisches) System bzw. einen anderen Prozess und das Experimentieren mit diesem Modell. Vgl. Brockhaus (LexiROM, 1995). 
und für die Ausbildung von militärischen Führungskräften eingesetzt wurde. Dieses Planspiel kann als unmittelbarer Vorläufer der heute üblichen wirtschaftlichen Planspiele gelten.

Das bekannteste und älteste (Plan-) Spiel ist sicherlich das Schachspiel. ${ }^{101}$ Zur Erreichung eines bestimmten Zieles - nämlich den Gegner "matt" zu setzen - stehen dem Spieler bestimmte Mittel zur Verfügung, die nach definierten Regeln eingesetzt werden können. Dabei ist das Ergebnis der Aktion des Spielers nicht nur vom eigenen, sondern auch vom Handeln des Gegenspielers abhängig. Dieser Sachverhalt ist für militärische Situationen typisch und trifft größtenteils auch für wirtschaftliche Verhältnisse zu. So hängt beispielsweise der Absatz und damit der Erfolg eines Unternehmens sowohl von dessen Entscheidungen als auch von den Entscheidungen der anderen Marktteilnehmer ab.

\subsubsection{Anwendungsgebiete von Planspielen}

Bei den Anwendungsgebieten von Unternehmenssimulationen kann im wesentlichen zwischen dem Einsatz in der betriebswirtschaftlichen Aus- und Weiterbildung und in der wissenschaftlichen Forschung sowie der Nutzung für unternehmerische Aufgabenstellungen differenziert werden.

Unternehmensplanspiele werden seit über 40 Jahren in der Aus- und Weiterbildung von Studierenden an Universitäten, Fachhochschulen und anderen Bildungseinrichtungen sowie zur Weiterbildung von Führungsnachwuchskräften in zahlreichen Unternehmen verwendet. Ziel ist dabei die praktische Anwendung von (betriebswirtschaftlichen) Kenntnissen in realitätsnahen Anforderungssituationen.

\footnotetext{
99 Vgl. Hartung (Fortbildung, 2000) Seite 48, Orth (Planspiele, 1999) Seite 5, Pack (Planspiele, 1968) Seite 8 oder Prehm (Marketingplanspiel, 1995) Seite 3.

100 Vgl. Koller (Planspieltechnik, 1969) Seite 95 oder Pack (Planspiele, 1968) Seite 10.

101 Schach (,Schah mat“: der König ist Tod), Brettspiel persisch-arabischer Herkunft. Das erste literarische Erzeugnis für die Existenz des Schachspiels ist ein mittelpersischer Roman aus der Zeit um 600 nach Christus. Vgl. Meyers (Lexikon, 1977).

102 Orth (Planspiele, 1999) Seite 12.
} 
Viele Praktiker beklagen sich über die zu theoretische Ausbildung in der Schule oder im Studium. Das Lernen in der Ausbildung orientiert sich nicht an den Anforderungen des Berufs. Dies zeigt sich insbesondere bei konkreten betrieblichen Problemstellungen. 103

Auf folgende Mängel in der Ausbildung weist die Literatur hin:

1. Lernen abstrakter und anwendungstechnischer Inhalte: Berufliche Anforderungssituationen sind - im Gegensatz zu wohldefinierten Übungsaufgaben in der Lehre mit einer eindeutigen Lösung - meist viel komplexer, nicht wohldefiniert und enthalten eine Vielzahl von Lösungsmöglichkeiten.

2. Zerfaserung der Lerninhalte: Durch die Aufteilung in einzelnen Fächern wird der Stoff in eine künstliche Ordnung gebracht, systematisiert gelehrt und abgefragt und entsprechend gelernt. Eine Integration von verschiedenen Aspekten in ein übergreifendes Problem nimmt man in der Ausbildung zwar selten vor, ist aber für eine erfolgreiche Anwendung des Wissens in der Praxis erforderlich.

3. Fehlen kooperativer Lernformen: In der Ausbildung stehen individuelle Leistungen im Vordergrund. Beurteilungen von Gruppenleistungen erschweren die Notengebung und werden entsprechend vermieden. Das Berufsleben ist jedoch durch ausgeprägte Teamarbeit gekennzeichnet, entsprechend werden Qualifikationen wie Kooperationsfähigkeit als grundlegend angesehen.

Dass mit Planspielen bestimmte Lernziele erreicht worden sind, zeigt Albach in einer empirischen Untersuchung. ${ }^{105}$ Danach wird während eines Spiels insbesondere die Fähigkeit ausgebildet,

1. unternehmerische Ziele und langfristige Konzeptionen zu formulieren,

2. risikoreiche Führungsentscheidungen zu analysieren und

3. aufeinander abgestimmte Entscheidungen in Führungsgruppen zu treffen.

Albach betont, dass der Prüfstein jedes Planspiels erst die Realität selbst ist, denn die eigentliche Bedeutung des Spiels liegt darin, dass die Managementschulung einen

\footnotetext{
103 Bloech et al. (Ausbildung, 1996) Seite 38.

104 Mandl et al. (Wissen, 1993) Seite 64 ff., und Bloech et al. (Weiterbildung, 1998) Seite 1.

105 Albach (Unternehmensspiele, 1974).
} 
Beitrag zur besseren Nutzung der Ressourcen des Unternehmens im Dienste der Gesellschaft leistet.

Weitere Lernziele sind Anwendung und Vertiefung von Fachwissen sowie das Erkennen inner- und zwischenbetrieblicher Zusammenhänge.

\subsubsection{Vor- und Nachteile von Planspielen}

Planspiele erfahren zur Zeit eine immer größere Verbreitung. Dabei wird oft betont, dass gerade mit dem Planspiel zahlreiche Vorteile einhergehen, die zu einer Verkürzung der vielfach kritisierten Distanz zwischen Theorie und Praxis führen. Die wichtigsten sind: 106

1. Komplexe und unübersichtliche Theorien können in ein vereinfachtes Modell übertragen werden.

2. Der besondere Charakter von unternehmerischen Entscheidungen wird verdeutlicht.

3. Die Teilnehmer werden dazu gebracht, ihre vorhandenen theoretischen Kenntnisse in praktische Fähigkeiten umzusetzen.

4. Die Bereitschaft zum Lernen und die Effektivität des Lernprozesses werden erhöht.

5. Die Beurteilung komplexer ökonomischer Situationen wird geschult.

Planspiele können als „sichere“ Spielwiese genutzt werden, um unterschiedliche Strategien mit einem Minimum an negativen Konsequenzen auszuprobieren. ${ }^{107} \mathrm{Ihr}$ Nutzen besteht dabei aber nicht in der Erstellung von detaillierten Plänen, die später unreflektiert umgesetzt werden, sondern vielmehr in der Validierung und gegebenenfalls Adaptierung des eigenen mentalen Modells über die Zusammenhänge in der Wirklichkeit. Die Einsicht, wie Ursache und Wirkung auseinanderfallen, führt dann bei geeignetem Einsatz zu einem besseren Verständnis des untersuchten Problems und in weiterer Folge zu einer raschen Reaktion auf Marktentwicklungen.

\footnotetext{
106 Vgl. Prehm / Ehlken (Planspiel, 1995) Seite 4 oder Puma (Spielanleitung, 1995) Seite 10 ff.

107 Vgl. Pederson (Simulation, 2000), Seite 102.

108 Vgl. Milling (Modeling, 1996) Seite 228.
} 
Insgesamt kann festgestellt werden, dass zur Unterstützung des Erwerbs einer guten Problemlösungs- und Handlungskompetenz für komplexe ökonomische Produktionsszenarien Unternehmensplanspiele eine wertvolle Ergänzung der Lehrinstrumente bieten. Weiterhin vermitteln sie betriebswirtschaftliches Faktenwissen und fördern das Zusammenhangswissen.

Daneben sind einige Nachteile von Planspielen zu nennen. Diese haben bislang den endgültigen Durchbruch des Planspiels als Lernmethode behindert. Unternehmensplanspiele können nicht 1110

1. qualitative Faktoren in einem ausreichenden Maße berücksichtigen,

2. alle möglichen Einflussgrößen auf wirtschaftliche Entscheidungen in dem Simulationsmodell verwenden,

3. die subjektiven Vorstellungen der Modellkonstrukteure ausschließen,

4. ohne relativ hohe Kosten gestaltet und durchgeführt werden,

5. alle generellen Schwächen der Modellerstellung ausschalten sowie

6. aufwendige Vorbereitungszeiten gänzlich verhindern.

Dazu gehören weiterhin die Überbewertung der Rationalität bei der Entscheidungsfindung, das Fehlen ernsthafter (realer) Verlustmöglichkeiten, der im Vergleich zur Realität zu leichte Zugang zu entscheidungsrelevanten Informationen und die Gefahr, dass bei den Spielern falsche Vorstellungen über die Abläufe in der Realität entstehen. ${ }^{111}$ Diese Spieler sind dann allerdings ohnehin leicht durch den ersten Eindruck beeinflussbar.

\footnotetext{
109 Vgl. dazu Bloech (Unternehmensplanspiele, 1999) Seite 408 und Bloech et al. (Weiterbildung, 1998) Seite 45.

110 Prehm / Ehlken (Planspiel, 1995) Seite 7.

111 Vgl. Prehm / Ehlken 1995, Seite 10.
} 


\subsection{Planspiele in der betriebswirtschaftlichen Aus- und Weiterbildung}

\subsubsection{Aufbau und Funktionsweise}

Bereits 1968 beschreibt Ludwig Pack den Begriff des Unternehmer-Planspiels. „Unternehmer-Planspiele kann man als eine dynamische Ausbildungs- und Übungsmethode bezeichnen, welche sich der modellhaften Darstellung konkreter wirtschaftlicher Situationen bedient. Das dem Planspiel zugrunde liegende Modell stellt eine Vereinfachung der Wirklichkeit dar, wobei der Grad der Vereinfachung durch den Zweck bestimmt wird, welchem das Planspiel zu dienen hat. Diese Vereinfachung der Wirklichkeit, die dadurch erfolgt, dass unbedeutende Eigenschaften weggelassen werden und die für das Betrachtungsziel wesentlichen Merkmale in den Blickpunkt gezogen werden, ermöglicht erst die rationale Durchdringung der verwickelten Zusammenhänge, die für das Wirtschaftsleben und die Unternehmungen typisch sind. Jedem Unternehmer-Planspiel liegt ein bestimmtes Modell zugrunde. Die für den Spieler wichtigen Teile des Modells werden in der Spielanleitung bekannt gegeben, allerdings nur insoweit, wie sie für das Fällen der Entscheidungen notwendig sind. Ferner enthalten diese Spielanleitungen Angaben darüber, welche Entscheidungen wann zu treffen sind und für welche Zeitperiode diese Entscheidungen gelten.“

Pack reduziert seine Ausführungen hierbei auf den für den Spieler sichtbaren Bereich, nämlich das Modell und die dazugehörige Spielbeschreibung.

Bleicher erweitert den Begriff, in dem er ein Unternehmensspiel in zwei Bereiche, den Aktionsbereich und den Reaktionsbereich (Simulationsmodell), unterteilt. 113

Dabei umfasst der Aktionsbereich bzw. Spielerbereich bestimmte Spielregeln. Dieser bildet für die Spieler die Grundlage, ihre Entscheidungen in Abhängigkeit der

112 Pack (Planspiele, 1968) Seite 11.

113 Bleicher (Unternehmensspiele, 1969) 
Vorperiodenergebnisse, der Orientierungsinformationen durch die Spielleitung und der eigenen Zielsetzung zu treffen.

Alle Spieler führen ihre Entscheidungen dem Simulationsbereich zu. Hier werden die Entscheidungen mit mathematischen und logischen Verknüpfungen zu Ergebnissen (Reaktionen) verarbeitet. Dabei kann der Leitungsbereich Einfluss auf die Entscheidungen durch Änderung bestimmter Parameter oder Regeln geltend machen.

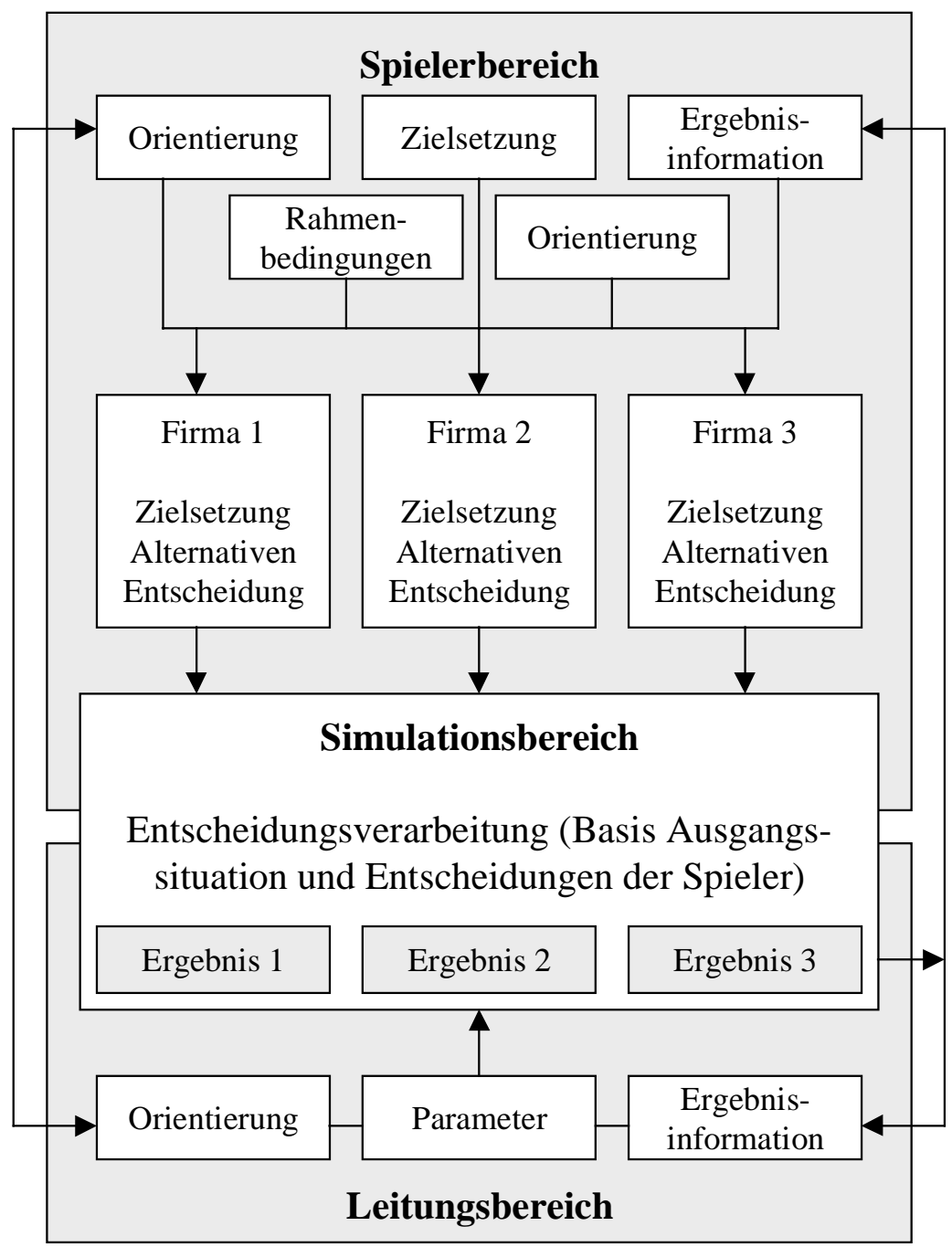

Abb. 6: Grundstruktur von Planspielen

114 Vgl. Bleicher (Unternehmungsspiele, 1969) Seite 166, Prehm (Marketingplanspiel, 1995) Seite 8, Schöllhammer (Planspiele, 1964) Seite 329. 
Zwischen den beiden Bereichen fließen zwei Informationsströme: Die Entscheidungsinformationen, die Entscheidungen aller Spieler umfassen, und die Ergebnisinformationen, die als Grundlage der neuen Entscheidungen dienen.

Darüber hinaus können Orientierungsinformationen, die weitergehende Daten über das zukünftige Verhalten des Simulationsmodells beinhalten, von der Spielleitung an den Spielerbereich (gegebenenfalls selektiv) gegeben werden.

Dem Unternehmensspiel liegt ein dynamisches Modell zugrunde, bei dem es im Zeitablauf zu einer Folge von Entscheidungen (Aktionen) und Ergebnisse (Reaktionen) kommt, die sich gegenseitig im Sinn einer Reaktionskette bedingen.

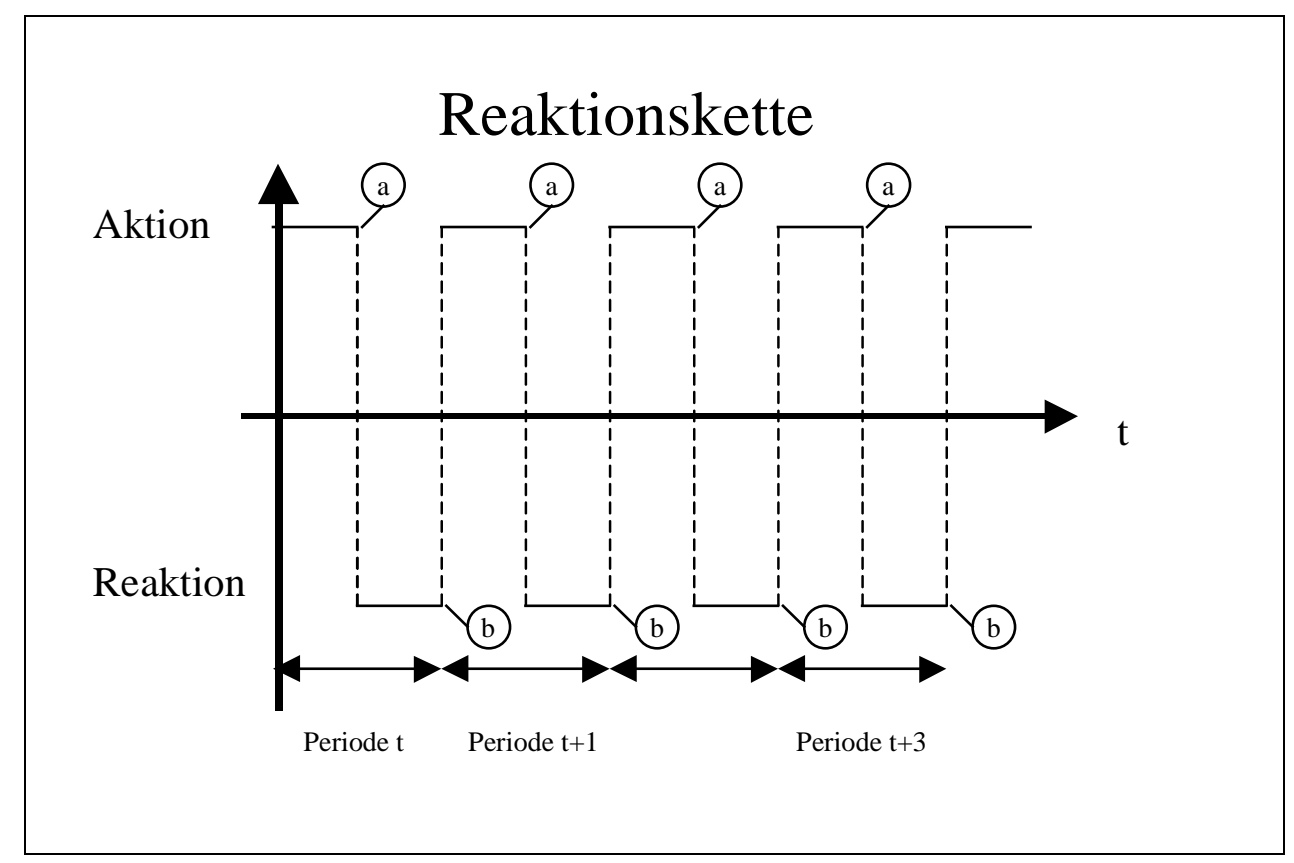

Abb. 7: Reaktionskette in Unternehmensspielen

An den Ecken (a) werden Entscheidungsinformationen und an den Ecken (b) Ergebnisund Orientierungsinformationen erzeugt. Diese bewirken jeweils die folgende Reaktion bzw. Aktion.

115 Prehm (Marketingplanspiel, 1995) Seite 10. 


\subsubsection{Arten und Einteilung von Planspielen}

In der Literatur gibt es bisher keinen umfassenden, theoretisch begründeten Klassifikationsansatz für ökonomische Planspiele. Nachfolgend sollen wesentliche Einteilungsmöglichkeiten kurz beschrieben werden. 116

1. Betrieblicher Umfang (funktional oder generell): Die Mehrzahl der über 2000 existierenden Planspiele $\sqrt{117}$ sind generelle Planspiele, die ein Unternehmen mit seinen wesentlichen Teilbereichen (z.B. Beschaffung, Produktion und Absatz) abbilden. Die speziellen oder funktionellen Planspiele beschränken sich hingegen auf die Darstellung ausgewählter Unternehmensbereiche.

Während spezielle Planspiele eher für die Ausbildung in Spezialgebieten geeignet sind, betonen die generellen Planspiele betriebswirtschaftliche Zusammenhänge und Interdependenzen sowohl zwischen den unternehmerischen Teilbereichen als auch zwischen einem Unternehmen und den Beschaffungs- und Absatzmärkten.

2. Konkretisierungsgrad (speziell oder allgemein): Man kann zwischen konkreten oder abstrakten Modellen unterscheiden. Konkret heißt hierbei, dass das oder die abgebildeten Unternehmen mit seinen Produkten und Märkten genauer beschrieben sind. Ziel ist hierbei, Know-how für das abgebildete in der Regel eigene Unternehmen zu generieren.

3. Interaktion zwischen den Teilnehmergruppen (Solospiel oder Konkurrenzspiel): Bei Solospielen ist nur ein Unternehmen bzw. eine Gruppe von Spielern involviert. Im Gegensatz dazu steht das Konkurrenzplanspiel, dass eher Wettbewerb unter den beteiligten Spielgruppen schafft. Für die Aus- und Weiterbildung sind gerade Konkurrenzplanspiele geeignet.

4. Determiniertheit des Modells (determiniert oder stochastisch): Mit stochastischen oder zufälligen Methoden oder Funktionen hat man die Möglichkeit, die Natur noch besser abzubilden. So sind z.B. ein Mitarbeiterstreik oder eine nicht erfolgte Anlieferung von Produktionsfaktoren zufällig abzubilden.

5. Regelgebundenheit: Hier wird zwischen starren oder freien Modellen unterschieden. In der Aus- und Weiterbildung herrschen die starren Modelle vor. Dies hat allerdings den Nachteil, dass meistens nur das „Wann“ und „Wie viel“

\footnotetext{
116 Orth (Planspiele, 1999) Seite $17 \mathrm{ff}$.

117 Drössler (Planspiele, 1998) Seite 83.
} 
entschieden werden kann. Das „Wie“ wird oder kann nicht in der Entscheidungsfindung berücksichtigt werden.

6. Zusammensetzung der Spielergruppen (Einzelpersonen oder Gruppen): Gruppenspiele bieten sich insbesondere dann an, wenn neben der reinen technischen Lösung auch soziale Kompetenzen wie Kooperations- und Kommunikationsfähigkeiten erlernt werden sollen. Ab einer bestimmten Anzahl von Entscheidungsvariablen ist jedoch mit mehr als einer Person zu arbeiten.

7. Nutzung rechnerischer Hilfsmittel (manuell oder Computer): Ursprünglich wurden Planspiele natürlich ohne Computer gespielt. Tabellen oder Diagramme simulierten die Unternehmung und die Märkte. Durch Fortschritte in der Computertechnologie haben sich sogenannte Maschinen- oder Computerspiele durchgesetzt. Die Berechnungen sind fast fehlerfrei und können schneller durchgeführt werden. Dadurch sind auch komplexere Modelle konstruierbar. Das Internet wird diesen Trend noch weiter verstärken.

8. Komplexität des Modells (hoch oder niedrig): Die Komplexität hängt von der Anzahl der benutzten Variablen sowie von den Verknüpfungen untereinander ab. Durch die Begrenzung von Variablen oder das teilweise Aus- oder Einschalten lässt sich der Komplexitätsgrad verändern.

Aus dem betrieblicher Umfang (Punkt 1) und dem Konkretisierungsgrad (Punkt 2) lassen sich drei unterschiedliche Typen von Planspielen ableiten: Generelle, funktionsorientierte und institutionelle Planspiele. 118

\subsubsection{Generelle Planspiele}

Generelle Planspiel haben keinen Fokus auf einen bestimmten Funktionsbereich. Sie zeichnen sich dadurch aus, dass sie alle wichtigen Unternehmensfunktionen wie z.B. Produktion, Absatz, Finanzierung und Personal enthalten.

118 Unter Institutionen versteht man verschiedene Wirtschaftssubjekte wie Banken, Versicherungen, Krankenhäuser oder Wohnungsunternehmen. 


\subsubsection{EpUS - Ein-Platz-Unternehmens-Simulation}

Das Planspiel EpUS $S^{199}$ ist ein vollständiges Unternehmensmodell. Der Spieler trifft für das Unternehmen in allen wichtigen Bereichen die Entscheidungen. Im Vordergrund stehen die betrieblichen Entscheidungsbereiche Beschaffung, Produktion und Absatz. Aufgabe ist es, den Unternehmenserfolg zu maximieren. Die vier Konkurrenten, die die gleiche Zielsetzung verfolgen, sind bereits fest eingegeben, die Qualität dieser Entscheidungen ist durchschnittlich. Die Entscheidungen aller fünf Unternehmen werden unter gleichen Bedingungen berechnet und ausgewertet.

Der Produktionsprozess ist einstufig. Aus zwei Rohstoffen werden zwei Fertigprodukte hergestellt. Es gibt einen Maschinentyp, dessen technische Daten den Personaleinsatz und den Verbrauch an Hilfs- und Betriebsstoffen festlegen.

Für den Absatzbereich ist zu entscheiden, zu welchen Preisen die beiden Produkte auf zwei unterschiedlichen (oligopolistischen) Märkten angeboten und welche Ausgaben für die jeweiligen absatzpolitischen Instrumente Werbung und Kundendienst getätigt werden.

Weiterhin können gegebenenfalls Marktforschungsinformationen (Preise, Umsatz und Absatzmengen der Konkurrenz) angefordert werden. Ferner sind Entscheidungen in den übrigen betrieblichen Bereichen Produktion, Beschaffung, Investition, Finanzierung und Personal zu treffen.

\subsubsection{BO-Cash}

Das (inzwischen kommerzielle) Konkurrenzplanspiel BO-Cash 120 ist am Lehr- und Entwicklungszentrum für quantitative Verfahren und Unternehmensplanspiele der Fachhochschule Bochum entstanden. Mehrere (meist vier) Unternehmen gehören derselben Branche an. Als Marktform liegt ein Angebotsoligopol vor, bei dem auf der Angebotsseite nur wenige, relativ große Verkäufer auftreten, während die

\footnotetext{
119 Vgl. dazu Epus (Spielanleitung, 1992) oder Bloech (Unternehmensplanspiele, 1999) Seite 409 ff..

120 Vgl. dazu BO-Cash (Spielanleitung, 2000).
} 
Nachfrageseite - mit vielen kleinen Käufern - nicht näher spezifiziert ist. Die Marktform des Angebotsoligopols bedeutet für jedes der vier Unternehmen, dass bei marktstrategischen Maßnahmen die vermutlichen Reaktionen der drei anderen Konkurrenten berücksichtigt werden müssen.

Die vier Unternehmen bieten jeweils drei verschiedene Produkte an, die durch folgende Merkmale gekennzeichnet sind:

\begin{tabular}{|l|l|}
\hline Produkt & Merkmale \\
\hline Produkt 1 & $\begin{array}{l}\text { Massenprodukt nach bewährter Technik, } \\
\text { seit längerer Zeit auf dem Markt }\end{array}$ \\
\hline Produkt 2 & $\begin{array}{l}\text { Dienstleistungsprodukt, } \\
\text { Verbreitung noch nicht überall gelungen }\end{array}$ \\
\hline Produkt 3 & $\begin{array}{l}\text { Produkt nach neuestem technischen Stand, } \\
\text { gewisse Marktstellung gesichert }\end{array}$ \\
\hline
\end{tabular}

Tab. 8: Produktmerkmale bei BO-Cash

Es wird davon abgesehen, die Produkte als konkrete Erzeugnisse zu definieren. ${ }^{121}$ Dies erscheint angebracht, damit die Anwendung des Unternehmensplanspiels für alle Branchen flexibel zugänglich bleibt. Bei den Produkten handelt es sich weder um komplementäre Produkte noch um substitutive Güter. Daher tauchen auch keine Substitutionseffekte auf, d.h. beispielsweise, dass die Abnehmer des Produktes 1 dieses nicht durch das Produkt 2 ersetzen können.

BO-Cash ist aufgrund seiner Komplexität und der Menge an Entscheidungen von einem Team zu spielen. Für jede Periode des Spiels, die in der Realität einem Zeitraum von drei Monaten entspricht, haben die Teams folgende Entscheidungen zu treffen:

121 Z.B. Waschmittel, Softwareprogramme oder Pocket-Kameras. 


\begin{tabular}{|l|l|}
\hline Absatz & $\begin{array}{l}\text { Verkaufspreise für drei Produkte auf je vier Märkten (gegebenenfalls auch } \\
\text { Sonderausschreibung) } \\
\text { Werbeausgaben für drei Produkte auf jeweils vier4 Märkten } \\
\text { Vertriebsausgaben für vier Märkte } \\
\text { Forschungsausgaben für drei Produkte } \\
\text { Versandmengen von drei Produkten für jeweils vier Märkte } \\
\text { Ankauf eines Marktforschungsberichtes (fakultativ) }\end{array}$ \\
\hline Einkauf & $\begin{array}{l}\text { Bestellung von zwei Rohstoffen } \\
\text { Bestellung als Handelsware für drei Produkte }\end{array}$ \\
\hline Produktion & $\begin{array}{l}\text { Herstellungsmengen für drei Produkte } \\
\text { Investitionen von drei Maschinentypen (fakultativ) } \\
\text { Desinvestitionen von drei Maschinentypen (fakultativ) } \\
\text { Bestimmen des Instandhaltungssatzes } \\
\text { Einstellung oder Entlassen von Fach- und/oder Hilfsarbeitern (fakultativ) } \\
\text { Festlegen der Sozialinvestitionen }\end{array}$ \\
\hline Finanzen & $\begin{array}{l}\text { Bestimmen des eigenen Zahlungsverhaltens } \\
\text { Aufnahme von kurz- und/oder langfristigen Krediten } \\
\text { Anlage von finanziellen Mitteln (Finanzinvestition) }\end{array}$ \\
\hline Sonstiges & Rationalisierung im Verwaltungsbereich \\
\hline
\end{tabular}

Tab. 9: Entscheidungen bei BO-Cash

Anhand der Variablen kann man erkennen, dass BO-Cash den Absatzbereich stark fokussiert.

Gemäß Aussage der Entwickler unterscheidet sich BO-Cash von anderen Unternehmensplanspielen vor allem durch ein Marktmodell, dass das heutige Marktgeschehen der Wirtschaftspraxis realistisch abbildet.

\subsubsection{Funktionsorientierte Planspiele}

Funktionsorientierte Planspiele legen den Schwerpunkt und damit die Vertiefung ihrer Modellbildung auf bestimmte betriebliche Funktionen. So gibt es u.a. Planspiele mit Schwerpunkt F\&E, Innovation, Logistik, Marketing, Produktion, Entsorgung oder Finanzierung. 


\subsubsection{PUMA - Produktions- und Marketing-Simulation}

Das Unternehmensplanspiel PUMA ${ }^{122}$ ist ein Konkurrenzspiel, an dem bis zu fünf Unternehmen beteiligt sind. Wesentliche Merkmale der Unternehmenssimulation sind die Produktion und der Absatz von Produkten. Je nach Gestaltung der Modellparameter kann sich die Produktion auf die Montage eines Fertigerzeugnisses beschränken oder einen mehrstufigen und mehrperiodigen Produktionsprozess mit verschiedenen Halberzeugnissen und unterschiedlichen Fertigerzeugnissen umfassen. Der Verkauf erfolgt über drei Absatzwege auf bis zu fünf Märkten. Durch die Gestaltung der vorhandenen Marketinginstrumente kann der Absatz beeinflusst werden.

Folgende Entscheidungen sind im Planspiel zu treffen:

\begin{tabular}{|l|l|}
\hline Material & $\begin{array}{l}\text { Einkaufsmengen mit zwei Lieferterminen (Rohstoffe, Halb- und Fertigerzeugnisse) } \\
\text { Notbeschaffung } \\
\text { Normalbeschaffung } \\
\text { Verkaufsmengen aller Faktoren und Erzeugnisse }\end{array}$ \\
\hline Investitionen & $\begin{array}{l}\text { Auswahl von Maschinentypen für die Produktion } \\
\text { Anzahl der Maschinen } \\
\text { Kauf oder Leasing }\end{array}$ \\
\hline Arbeitskräfte & $\begin{array}{l}\text { Einstellungen / Entlassungen } \\
\text { Lohnangebot } \\
\text { Einstellung von Aushilfskräften }\end{array}$ \\
\hline Produktion & $\begin{array}{l}\text { Produktionsmengen je Produkt (Halb- und Fertigerzeugnisse) } \\
\text { Maschinenschaltung: Zeit- (0 - 125 \%) oder Leistungsschaltung (50 - 150 \%) }\end{array}$ \\
\hline Finanzen & $\begin{array}{l}\text { Kreditaufnahme } \\
\text { Finanzanlage } \\
\text { Transferzahlung an Unternehmen } \\
\text { Kapitalerhöhung durch Aktionäre }\end{array}$ \\
\hline $\begin{array}{l}\text { Entscheidungen je Produkt und je Markt: Verkaufspreis (Filiale und Handel), } \\
\text { Händlereinkaufspreis, Werbung, Kundendienst, Verkaufsförderung, } \\
\text { Verkaufsmengenlimit (Filiale und Handel), Informationsbeschaffung } \\
\text { Earketing }\end{array}$ & $\begin{array}{l}\text { Entscheidungen je Produkt: Qualitätsverbesserung, Forschung \& Entwicklung, } \\
\text { Entscheidungen je Markt: Investitionen in das Filialnetz } \\
\text { Sonstige Entscheidungen: Beteiligung an Ausschreibungen, Ausführen von Aufträgen }\end{array}$ \\
\hline
\end{tabular}

Tab. 10: Entscheidungen bei PUMA

Interessant ist bei Puma die Möglichkeit der Notbeschaffung. Es besteht für jeden Spieler die Möglichkeit, auch nach abgeschlossener Planung der Periode nachträglich Rohstoffe einzukaufen. Diese Bestellungen verursachen allerdings höhere Kosten, was

${ }^{122}$ Vgl. dazu Puma (Spielanleitung, 1992) oder Bloech (Unternehmensplanspiele, 1999) Seite 415 ff. 
sich negativ auf die Ertragssituation aber positiv auf die Auslastung der Produktionskapazitäten auswirkt.

\subsubsection{SIM-LOG - Simulation zur Logistik}

Der Schwerpunkt von SIM-LOG ${ }^{123}$ liegt in der Material- und Qualitätswirtschaft. In einem Kuppelprozess werden Haupt- und Nebenprodukte in einem festen Verhältnis erzeugt (z.B. Kraftwerk: Strom und Wärme; oder Sägewerk: Bretter und Späne). Die Anzahl der verkaufsfähigen (guten) Hauptprodukte hängt von der Qualität der eingesetzten Rohstoffe ab. Schlechte Rohstoffqualitäten bedeuten einen höheren Anteil an minderwertigen Produkten, die nur zu deutlich geringeren Preisen zu verkaufen sind. Die Qualität der eingesetzten Rohstoffe kann durch Beschaffungsmaßnahmen (Auswahl geeigneter Lieferanten) und / oder durch eigene Qualitätskontrollmaßnahmen (Rohstoffprüfung vor dem Verbrauch) angehoben werden. Beide Maßnahmen verursachen Kosten. Einer höheren Rohstoffqualität steht als Ergebnis der Fertigung eine größere Anzahl guter verkaufsfähiger Produkte gegenüber. Die Maßnahmen Einkaufsqualität und Qualitätskontrolle sind so abzustimmen, dass sich ein möglichst günstiges Unternehmensergebnis einstellt.

Die Erzeugnisse werden auf einem oligopolistischen Markt angeboten. Die Unternehmen bestimmen die Preishöhe und die Ausgaben für Marketing (z.B. Werbung) selbst.

Das Unternehmensergebnis hängt von den Größen Beschaffungsmaßnahmen, Qualitätskontrolle, Produktionsvolumen, Preissetzung und Marketingeinsatz ab. Entscheidungen:

123 Vgl. dazu Simlog (Spielanleitung, 1998) oder Bloech (Unternehmensplanspiele, 1999) Seite $411 \mathrm{ff}$. 


\begin{tabular}{|l|l|}
\hline Rohstoff & $\begin{array}{l}\text { Entscheidungen, welcher der vier Rohstoffe in welcher Menge bei } \\
\text { welchem der vier Lieferanten bestellt werden soll } \\
\text { Festlegung eventueller Notbeschaffung (zu erhöhten Kosten und } \\
\text { gegebenenfalls geringerer Qualität) } \\
\text { Festlegung der Transportart: Linie (Fix- und Stückkosten) oder Charter } \\
\text { (fixe Kosten je Charge) }\end{array}$ \\
\hline Finanzierung & $\begin{array}{l}\text { Kreditaufnahme oder Rückzahlung } \\
\text { Kredittilgung oder Finanzanlage }\end{array}$ \\
\hline $\begin{array}{l}\text { Qualitätsverbesserung } \\
\text { aller Rohstoffe }\end{array}$ & $\begin{array}{l}\text { Entscheidung, um wie viel Prozent die Qualität durch Aussortieren } \\
\text { verbessert werden soll (fixe und variable Kosten) }\end{array}$ \\
\hline Maschinen & Kauf oder Verkauf von Maschinen \\
\hline Produktion & Mengenentscheidung über Haupt- und Nebenprodukt \\
\hline Absatz & $\begin{array}{l}\text { Entscheidung für Verkaufspreis, Werbeaufwand und Exportmenge } \\
\text { für Haupt- und Nebenprodukt }\end{array}$ \\
\hline Information & Beschaffungs- und Absatzinformationen (Marktforschung) \\
\hline
\end{tabular}

Tab. 11: Entscheidungen bei SIM-LOG

Analysiert man die Beschreibung und die Entscheidungsmöglichkeiten bei SIM-LOG, erscheint einem der Name nicht eindeutig genug. Treffender wäre nach Ansicht des Autors der Name „Sim-EProQ - Simulation Einkauf und Produktqualität“.

\subsubsection{Institutionsorientierte Planspiele}

Institutsorientierte Planspiele bilden die speziellen Problemstellungen ab, denen sich Institutionen stellen müssen. So gibt es z.B. spezielle Versicherungs-, Bank-, Verwaltungs-, Krankenhaus- oder Planspiele für die Entsorgungswirtschaft.

\subsubsection{PENTA - Planspiel Entsorgungs- und Abfallwirtschaft}

Das Planspiel PENTA ${ }^{124}$ ist ein allgemeines Unternehmensplanspiel mit allen wesentlichen betrieblichen Teilbereichen (Beschaffung, Produktion, Absatz, Finanzierung) und dem Schwerpunkt in den Bereichen Entsorgungs- und Abfallwirtschaft.

124 Vgl. dazu Penta (Spielanleitung, 1991) oder Bloech (Unternehmensplanspiele, 1999) Seite 418 ff. 
Im Produktionsprozess fallen je nach Erzeugnismenge und gewählten Verfahren folgende Abfälle bzw. Emissionen in unterschiedlichem Ausmaß an: Lärmentwicklung, Luftverschmutzung, Wasserverunreinigung, fünf verschiedene, feste Abfallstoffe und Sonderabfälle (nach Recycling bzw. Verbrennung). Im Rahmen der Abfall- und Entsorgungswirtschaft sind diese Abfälle und Emissionen innerhalb von gegebenen Grenzwerten bzw. Höchstmengen (gesetzliche Vorschriften) und unter Berücksichtigung von Bußgeldern zu behandeln.

Die Unternehmen haben dabei folgende Möglichkeiten, ihre anfallenden Emissionen zu entsorgen:

\begin{tabular}{|l|l|}
\hline Eigene Entsorgung & $\begin{array}{l}\text { Lärmdämmung (Reduzierung des Produktionslärms) } \\
\text { Luftfilter (Reduzierung der Luftverunreinigungen) } \\
\text { Wasseraufbereitung (Abwasserbehandlung in Kläranlagen) } \\
\text { Recycling (Rückgewinnung von Rohstoffen aus den festen Abfallstoffen) } \\
\text { Verbrennung (Verdichtung der Sonderabfälle nach Recycling) } \\
\text { Deponierung (Lagerung der Sonderabfälle nach Verbrennung bzw. } \\
\text { Recycling) }\end{array}$ \\
\hline Fremdentsorgung & $\begin{array}{l}\text { Abgabe der festen Abfallstoffe, Wasser und Sonderabfall an eine } \\
\text { Börse, d.h. Entsorgung durch Fremdbetriebe (das sind auch am } \\
\text { Planspielbeteiligte Konkurrenten). }\end{array}$ \\
\hline $\begin{array}{l}\text { Abgabe an die Umwelt } \\
\text { (,,wilde Entsorgung“) }\end{array}$ & $\begin{array}{c}\text { Abgabe aller Abfallstoffe und Emissionen ohne weitere Behandlung } \\
\text { an die Umwelt. Bei der Überschreitung von Grenzwerten werden }\end{array}$ \\
& Bußgelder verhängt bzw. erfolgen Produktionskürzungen. \\
\hline $\begin{array}{l}\text { Fremdversorgung } \\
\text { (Entsorgung für Dritte) }\end{array}$ & $\begin{array}{c}\text { Zur besseren Auslastung der eigenen Entsorgungsanlagen kann für } \\
\text { Dritte (auch direkte Konkurrenten) eine Entsorgung bzw. } \\
\text { Aufbereitung für Wasser, feste Abfallstoffe und Sonderabfall } \\
\text { durchgeführt werden. }\end{array}$ \\
& \begin{tabular}{l} 
Der Bezug der Abfallstoffe erfolgt über die Abfallbörse. \\
\hline
\end{tabular} \\
\hline
\end{tabular}

Tab. 12: Entscheidungen bei PENTA

\subsubsection{KLIMA ${ }^{\text {FORTE }}$ - Klinikmanagement mit Fallpauschalen-Finanzierung}

Ziel dieses Planspiels ist es, die veränderten Anforderungen an das Krankenhausmanagement abzubilden: Ein neues Preissystem für die Finanzierung der Betriebskosten der Krankenhäuser wurde eingeführt, öffentlich-rechtliche Krankenhäuser dürfen Überschüsse erwirtschaften (mit dem Risiko der Schließung bei hohen Verlusten) und die von vielen Krankenhäusern neuerdings angenommene Rechtsform der gemeinnützigen $\mathrm{GmbH}$ und (in Bayern) des Selbständigen 
Kommunalunternehmens vergrößern die für das Krankenhausmanagement nutzbaren Entscheidungsspielräume. Hinzu kommt die Möglichkeit, unerlässliche, im Verhältnis $\mathrm{zu}$ Diagnose und Therapie aber periphere Aufgaben von externen Dienstleistern erledigen $\mathrm{zu}$ lassen (Outsourcing) und - was unter Umständen für die Versorgungssicherheit einer Region kritischer zu sehen ist - die gewinnorientierte Optimierung des Behandlungsprogramms.

Mit KLIMA ${ }^{\text {FORTE }}$ werden ca. acht Perioden (entsprechend acht Quartalen) des Lebens eines Krankenhauses gespielt, damit die Spieler, d.h. die Mitglieder des Vorstands dieses Krankenhauses, die jeweils hinzugewonnenen Erkenntnisse immer wieder zielorientiert einsetzen können.

$\mathrm{Zu}$ Beginn besteht das von einer Spielergruppe $\mathrm{zu}$ übernehmende KLIMA $^{\text {FORTE }}$ Krankenhaus aus drei Fachabteilungen, in denen Patienten unterschiedlicher Fallklassen behandelt werden. Die im Planspiel versorgbaren Fallklassen entsprechen den ICDKlassen (International Classification of Diseases).

Die Spieler können verschiedene krankenhaustypische Entscheidungen treffen: Sie weichen z.B. von der medizinisch nötigen Verweildauer ab, wandeln das Krankenhaus in eine Spezialklinik mit nur mehr einer Abteilung um, richten Zimmer für Notfallpatienten ein, setzen einen Hygienebeauftragten ein oder legen den medizinischen Sachmitteleinsatz je Fachabteilung und den Sachmitteleinsatz für die hotelmäßige Versorgung je Patient und Aufenthaltstag fest.

Interessant ist insbesondere, dass jede Spielgruppe vor Beginn des Spiels individuell Ziele vorgeben kann. So sind in einer Zielsystem-Maske 10 Punkte auf 10 Ziele zu verteilen und daher eine Gewichtung zu erreichen: Niedrige Fallkosten, hohe Gesamtqualität, hoher Spezialisierungsgrad, hohe Patientenzahl, angemessene Notfallkapazität, niedrige Abweisungsrate, hohe Patientenzufriedenheit, hoher Überschuss, hoher Belegungsgrad und niedrige Verweildauer.

125 Schwandt (Planspiel, 1998). 


\subsubsection{Anwendungsformen von betriebswirtschaftlichen Planspielen}

Im betriebswirtschaftlichen Bereich ergeben sich vor allem drei große Anwendungsgebiete für Computer-Simulationen. Einmal ist ihr Einsatz dann angezeigt, wenn Entscheidungsprobleme zwar quantifizierbar sind, mathematische Optimierungsverfahren jedoch nicht zur Verfügung stehen. Durch systematisches Variieren der Entscheidungsparameter kann im Wege der Computer-Simulation eine an ein Optimum angenäherte Lösung gefunden werden. Die Computer-Simulation bietet weiter die Möglichkeit, die Interaktion zwischen menschlichen Entscheidungen und der Entscheidungsumwelt in Form von Planspielen so realistisch wiederzugeben, dass sie zur Ausbildung von Führungskräften herangezogen werden kann. Gleichzeitig ist es aber auch möglich, das Verhalten der Entscheidungsträger unter den simulierten Bedingungen zum Gegenstand der Forschung zu machen. Schließlich wird diese Technik noch als Forschungsinstrument in der Organisationstheorie eingesetzt, um eine Anzahl einfacher Hypothesen zu komplexen Erklärungs- und Prognosesystemen zu verbinden. 126

Die für die betriebswirtschaftliche Ausbildung in Frage kommende Software lässt sich idealtypisch in drei Hauptgruppen einteilen. 127 Planspiele fallen dabei in die dritte Gruppe, dort in den Bereich Simulationsprogramme.

1. Kommerzielle Software unterteilt man in Systemsoftware, Anwendungssoftware, Endbenutzerwerkzeuge, Zusätze für Endbenutzer und Wissensbestände.

2. Eingeschränkte kommerzielle Software wird für Schulen und Ausbildung benutzt. Man unterscheidet in eingeschränkte kaufmännische Anwendungsprogramme mit weniger Programmfunktionen, eingeschränkte Endbenutzerwerkzeuge (,Juniorversionen“) und kommerzielle Software mit Schulrabatt.

3. Schulsoftware für kaufmännische Lernprozesse nennt man auch Teachware. Sie wird unterschieden in Drill \& Practice, Lernprogramme, Simulationsprogramme und wissensbasierte Lehrsysteme.

\footnotetext{
126 Koller (Planspieltechnik, 1969) Seite 5.

127 Vgl. Achtenhagen et al. (Lernhandeln, 1992) Seite $132 \mathrm{ff}$.
} 


\section{Konzeption des Planspiels LogisticPLUS}

Das dem Planspiel zugrundeliegende Modell stellt eine Vereinfachung der Wirklichkeit dar, wobei der Grad der Vereinfachung durch den Zweck bestimmt wird, welchem das Planspiel zu dienen hat.

Ludwig Pack

\subsection{Grundlagen}

\subsubsection{Zielsetzung von LogisticPLUS}

Der wichtigste Punkt bei der Konzeption eines Planspiels ist es, sich über die Ziele, die mit dem Planspiel erreicht werden sollen, im klaren zu sein. Eindeutige Ziele haben direkten Einfluss auf Existenz, Darstellung und Definition von bestimmten Modulen bzw. Funktionen.

Das übergeordnete Ziel des Planspiels LogisticPLUS ist die Aus- und Weiterbildung von Logistikern. Der Schwerpunkt liegt hierbei in der Fähigkeit des Spielers, bestimmte Produktmengen $\mathrm{zu}$ marktgerechten (minimalen) Kosten $\mathrm{zu}$ produzieren. Logistische Entscheidungen spielen dabei eine wesentliche Rolle. Der Spieler hat dabei die Möglichkeit, die Zusammenhänge bzw. Auswirkungen von „guten“ und „schlechten“ Entscheidungen auf die Ertragssituation der Unternehmung zu erkennen.

Um diesem Ziel gerecht zu werden, sind folgende (Basis-) Funktionen einer Unternehmung abzubilden: Marketing, Absatz, Beschaffung, Lagerhaltung, Produktion und Rechnungswesen. Hierbei wird im technischen Bereich ${ }_{129}$ bereits ein Schwerpunkt

129 Beschaffung, Lagerhaltung und Produktion. 
auf die logistischen Funktionen gelegt: der Leistungsfluss des Unternehmens ist mit typischen logistischen „Problemen“ gespickt. So ist z.B. die Fertigung mehrstufig, die Beschaffung erfolgt zeitversetzt und es findet ein Recyclingprozesse von Ausschussware statt. Im Bereich des Rechnungswesens - dieser umfasst insbesondere Kostenrechnung, Bestandsbewertung und Liquidität - ist bei der Definition der Funktionen ebenfalls auf das übergeordnete Ziel zu achten: die Auswertungen und Analysewerkzeuge für die Lösung der logistischen Fragestellungen müssen hinreichend ausgeführt sein. Weiterhin sind die zentralen Funktionen Personal, F\&E, Finanzierung und Qualität abzubilden.

\subsubsection{Das Unternehmen}

Jedes der fünf Unternehmen ist ein klassischer Produktionsbetrieb. Jedes Unternehmen befindet sich im Inland bzw. auf dem Inlandsmarkt, die Standort- und Transportkostenfaktoren sind für jedes Unternehmen gleich.

Jedes dieser fünf Unternehmen erzeugt zwei Halbfabrikate in zwei unterschiedlichen Produktionsprozessen (Produkt 1 und 2). Produkt 1 kann bis $\mathrm{zu} 30$ Prozent mit recyceltem Material aus vorhandenem Ausschuss kombiniert werden. Mit zwei zugekauften Halbfabrikaten (Produkt 3 und 4) können insgesamt zwei unterschiedliche Verkaufsartikel produziert werden: Singulus ist ein Kunststoffrohr, Combilus ein sanitäres Vorwandinstallationssystem.

Neben der Produktionssteuerung ist die Bereitstellung von Lager- und Transportkapazitäten zu gewährleisten. Lagerplätze fallen für die beiden Rohstoffe, für die vier Halbfabrikate, für die Verkaufsartikel, für das Ausschusslager und für Recyclat an.

\footnotetext{
130 In einer weiteren Version des Planspiels ist es angedacht, die fünf Unternehmen mit unterschiedlichen Distanzen und damit unterschiedlichen Transportkosten auf die einzelnen Märkte zu konfrontieren.

131 Singulus und Combilus werden in Kapitel 4.3.2 detailliert beschrieben.
} 
Neben sieben verschiedenen innerbetrieblichen Transporten zwischen Produktions- und Lagerstätten, die mit Gabelstaplern und Hilfspersonal erledigt werden müssen, müssen Lastkraftwagen für den Absatz bereitgestellt werden.

Mittels Einstellungen von Personal und Investitionen in verschiedene Produktionsmaschinen ist es möglich, weitere Kapazitäten zu schaffen. Dies ergäbe einen Wechsel in den 2- oder 3-Schichtbetrieb. Überstunden bei Maschinen und Personen sind teurer als reguläre Arbeitsstunden.

Jedes Unternehmen trifft seine Entscheidungen für eine zweimonatige Periode. Nach jeder Periode erhält jedes Unternehmen verschiedene Finanz-, Produktions-, Bestandsund Absatzberichte. Je nach Lernziel bzw. Marktverlaut ${ }^{132}$ dauert ein Spiel 8 bis 12 Perioden.

Da das Planspiel mit seinen 52 Entscheidungsvariablen von einem einzelnen Spieler kaum mehr zu überblicken und damit zu steuern ist, ist es sinnvoll, fünf Gruppen mit jeweils drei Spielern zu bilden. Jedes LogisticPLUS-Unternehmen wird unter funktionsbezogenen Gesichtspunkten in die Bereiche Produktion, Vertrieb und Service aufgeteilt. Durch die Bildung von Spielergruppen für die Unternehmungen werden Gespräche und Diskussionen über die Situation der Unternehmung angeregt.

132 Je nach Vorgabe der Spielleitung ist eine unterschiedliche Nachfrage und damit unterschiedliches Marktverhalten möglich. So kann das Marktvolumen steigen, in Wellen schwanken, gleich bleiben oder sinken. Weiterhin sind unterschiedliche Startbedingungen denkbar. So muss nicht zwingend von gleichen Startparametern aller fünf Unternehmen ausgegangen werden. Siehe hierzu auch Kapitel 6.1. 


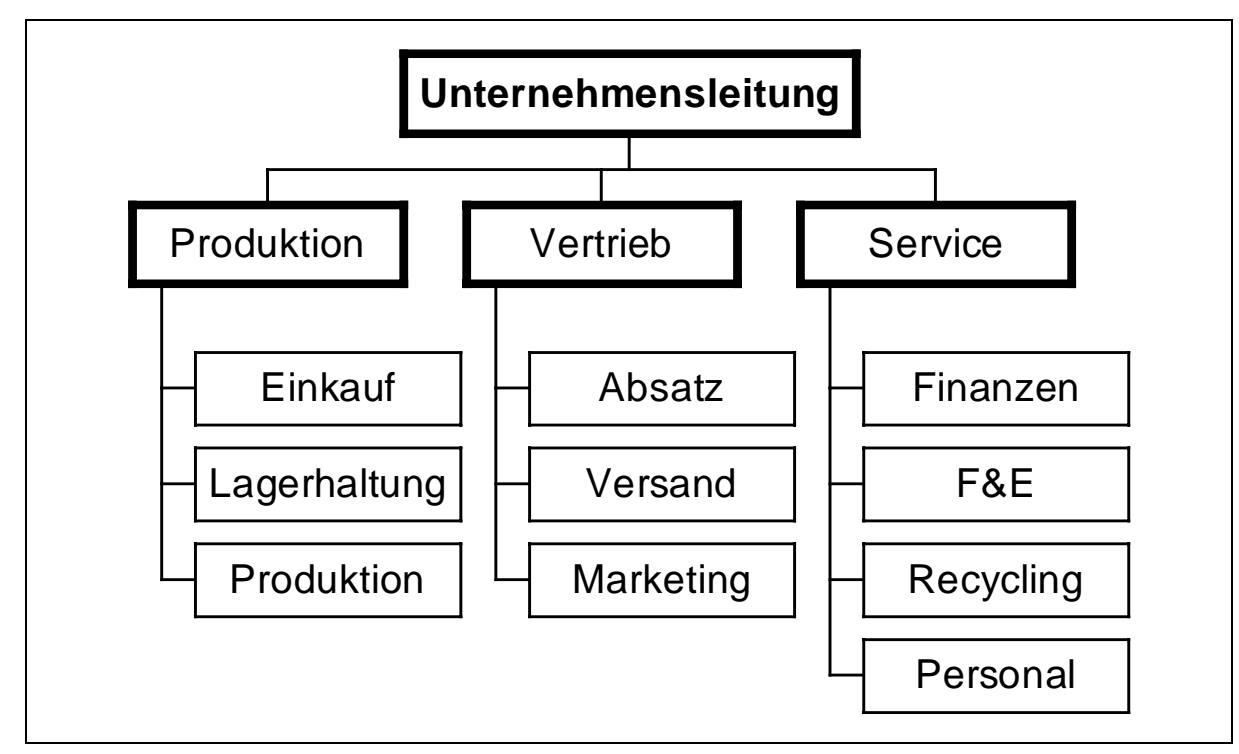

Abb. 8: Struktur (Beispiel) des LogisticPLUS - Unternehmens

Eine Dreiteilung in Produktion, Vertrieb und Service hat der Vorteil, dass sich die Gruppe aufteilen und sich somit jeder Spieler auf einen bestimmten Bereich konzentrieren kann. Dadurch können die komplexen Zusammenhänge schneller erkannt und somit bessere Entscheidungen getroffen werden. Eine alternative Aufteilung in zwei Bereiche ist möglich.

\subsubsection{Marktstellung und Wettbewerber}

Die fünf Unternehmen starten alle mit der gleichen Ausgangsposition. Jedes Unternehmen hat anfangs die gleiche Kosten- und Absatzstruktur. Der Marktanteil auf dem Inlandsmarkt beträgt 20 Prozent, hier konkurrieren die fünf Unternehmen untereinander. Es gibt keine weiteren Wettbewerber (Oligopol). Auf dem Exportmarkt besitzt jedes Unternehmen zu Beginn einen Marktanteil von 8 Prozent. Hier ist eine unbekannte Anzahl weiterer (virtueller) Konkurrenten $\frac{133}{133}$ vorhanden. Durch diese weiteren Unternehmen verringert sich u.a. der Einfluss der Werbeausgaben oder der eigene Einfluss auf den Marktdurchschnittspreis. Als dritter Markt kann durch die

133 Die Konkurrenten auf dem Exportmarkt sind virtuell, d.h. sie existieren ausschließlich im Modell der Spielleitung. 
Spielleitung eine Ausschreibungen veranlasst werden. Diese treten unregelmäßig auf und sind ausschließlich für die fünf am Planspiel teilnehmenden Unternehmen gedacht.

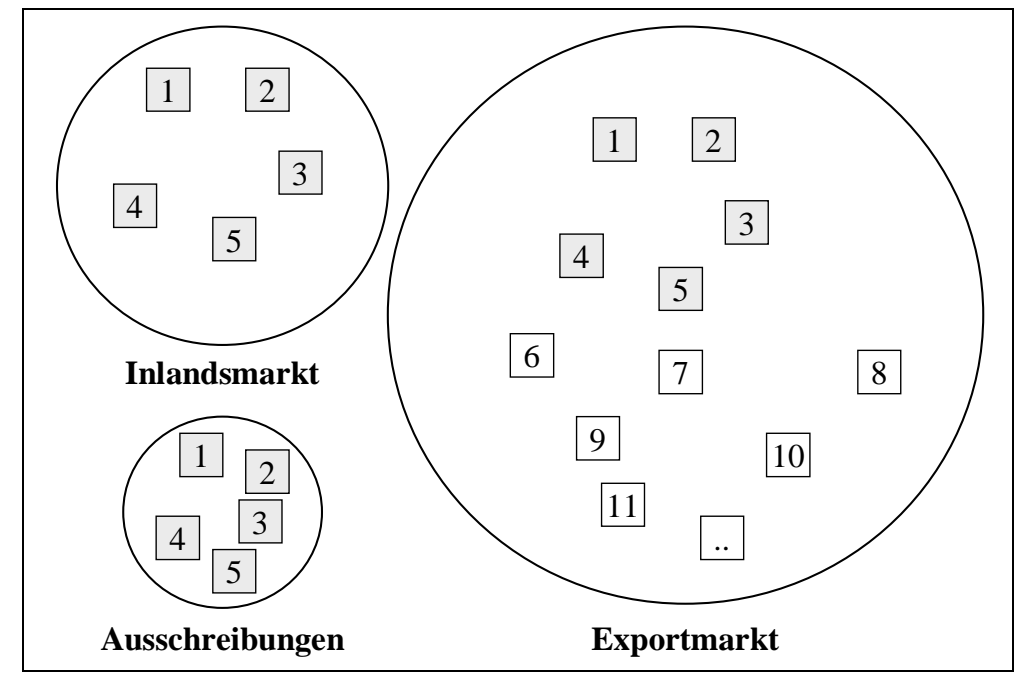

Abb. 9: Marktsituation bei LogisticPLUS

Die Quadrate stellen die einzelnen Unternehmen dar, die fünf direkt am Planspiel beteiligten Gruppen sind grau hinterlegt. Die nicht hinterlegten Quadrate sind eine für die Spieler nicht erkennbare Anzahl von Konkurrenten. Die Größe der drei Kreise verdeutlicht das unterschiedliche Volumen der Märkte. 


\subsubsection{Entscheidungen bei LogisticPLUS}

Die insgesamt 52 Entscheidungsvariablen der Spieler lassen sich wie folgt gruppieren:

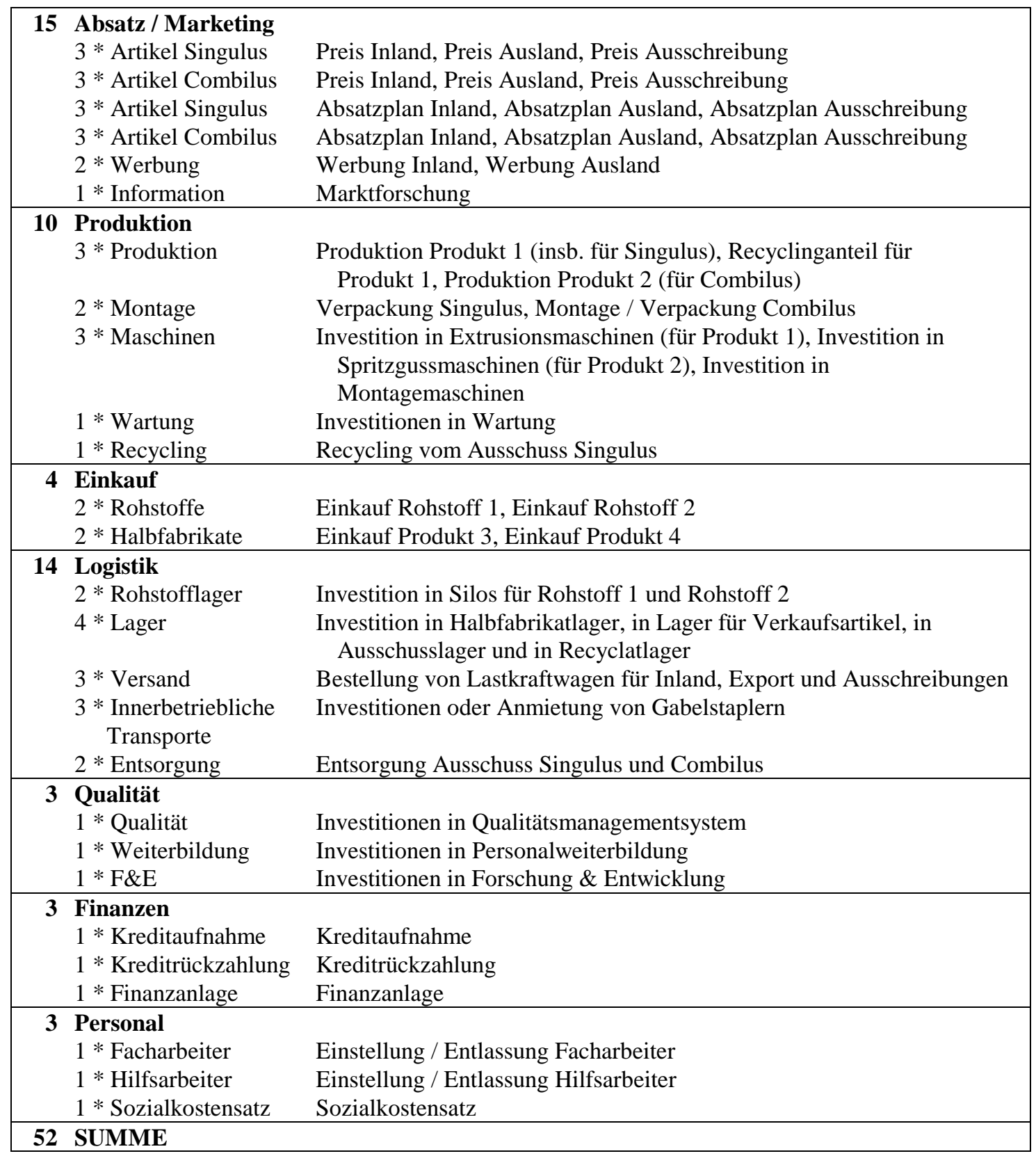

Tab. 13: Entscheidungen LogisticPLUS

In jeder Periode $\mathrm{t}$ werden in jedem der vier Unternehmensbereiche Beschaffung, Produktion, Montage und Vertrieb Entscheidungen getroffen. Diese haben direkte 
Auswirkungen auf die nächste Periode: Aufgrund der beschafften Rohstoffmenge ist z.B. nur eine bestimmte Produktionsmenge realisierbar. Als Währung wird der Euro (€) verwendet. Eine Periode dauert 2 Monate.

Die Fertigungs- oder Transportkapazitäten umfassen immer auch Stillstands- oder Ausfallzeiten.

\subsubsection{Marktmodell und ausführbare Computerprogramme}

Die Spielleitung simuliert die existierenden Märkte (Inland, Export, Ausschreibungen). Nachdem die Entscheidungsdaten aller fünf Spieler eingelesen wurden, erfolgt die Zuweisung von Marktvolumen, Durchschnittspreisen und (theoretisch möglichen) Absatzmengen an die einzelnen Spielern.

Die Spielleitung hat direkten Zugriff auf alle Spielerdaten und kann so einzelne Veränderungen vornehmen. Mittels Veränderung des Marktvolumens kann die Konjunktur, mittels Änderung der Beschaffungspreise für Produktionsgüter oder der Transportkosten kann direkter Einfluss auf die Erträge und Aufwendungen genommen werden. Weiterhin ist denkbar, dass die Spielleitung einige Spieler durch die Verzögerung der Anlieferung von Halbfabrikaten oder durch die Vernichtung eines Anteils im VK-Artikellager in deren nächster Spielperiode negativ beeinflusst.

Das Planspiel LogisticPLUS besteht aus zwei Programmen bzw. Modulen: einem Spielermodul und einem Spielleitermodul. Beide Module sind in Visual Basic for Applications unter Microsoft Excel 97 in Windows 95 bzw. in Windows NT Umgebung programmiert worden.

Das Spielermodul, im nachfolgenden PLANSPIEL ${ }^{134}$ genannt, simuliert eines der fünf Unternehmen. ${ }^{135}$ Alle Variablen aus der obigen Tabelle können durch den Vorstand bzw. den Spieler aktiv beeinflusst bzw. gesteuert werden. Aus diesen Variablen ergeben

\footnotetext{
${ }^{134}$ Zum besseren Verständnis und für die bessere Lesbarkeit wird nicht der echte Dateiname (LPLUSDAT.xls) sondern das Wort PLANSPIEL angegeben.

135 Insgesamt existieren somit fünf Spielermodule.
} 
sich für das Unternehmen bestimmte Kennwerte (z.B. Produktions- und Absatzzahlen, Lager-, Kassen- und Personalbestände, etc.). Die Unternehmen müssen versuchen, ihre Produkte auf den vorhandenen Märkten abzusetzen.

Die Oberfläche des Spielermoduls soll selbsterklärend sein. Die Bereiche der folgenden Abbildung können von den Spielern einzeln aufgerufen werden.

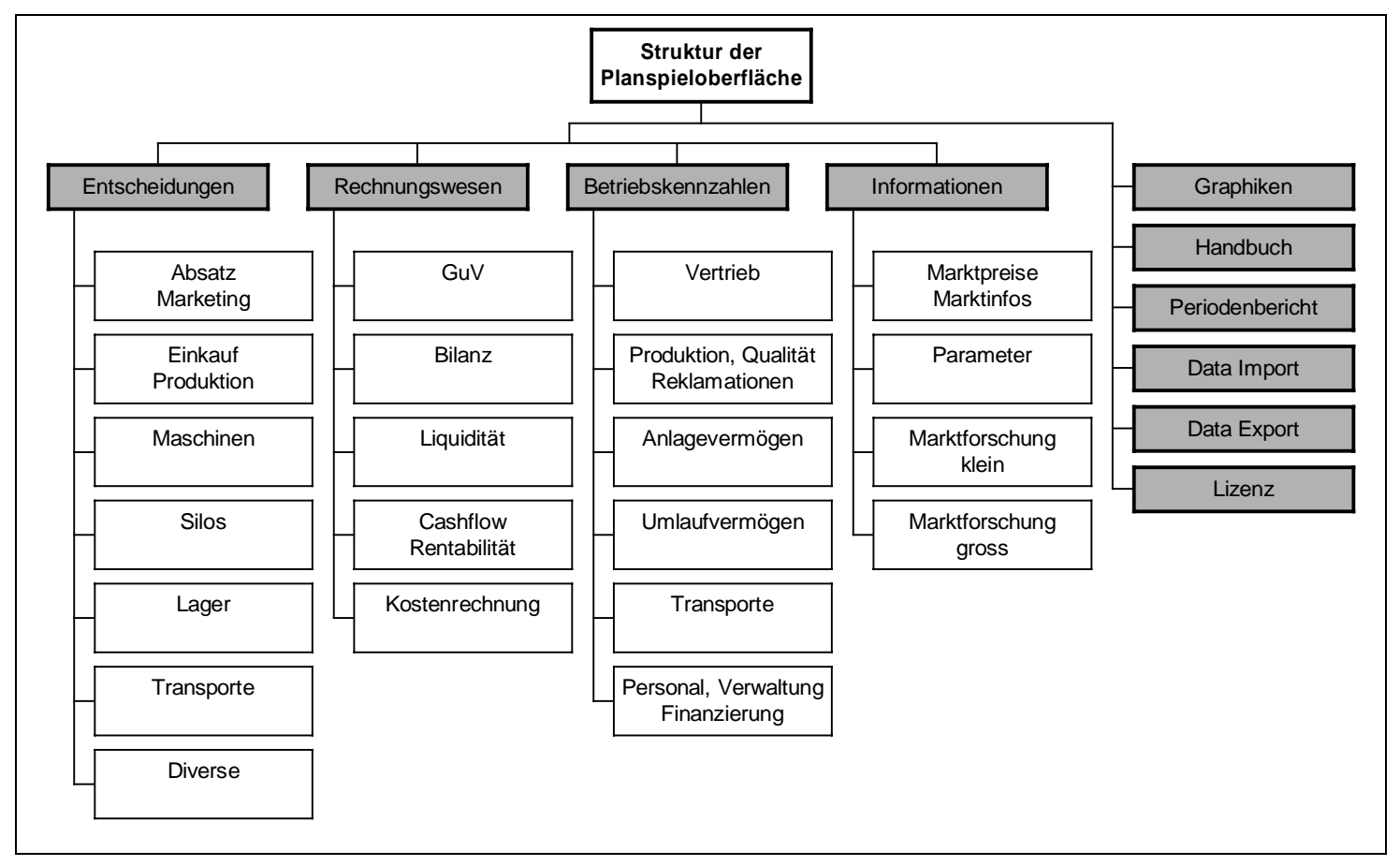

Abb. 10: Struktur der Planspieloberfläche

Das Planspiel unterscheidet vier Hauptbereiche und sechs Nebenbereiche, die in obiger Darstellung grau hinterlegt sind. Für jeden der Bereiche liegt eine eigene Computermaske vor, die im Kapitel 4.3.7 detailliert beschrieben werden.

Im Hauptbereich Entscheidungen müssen durch den Spieler alle 52 Entscheidungsvariable je Spielrunde festgelegt werden. Im Rechnungswesen erhält der Spieler finanzielle Informationen, die durch seine und die Entscheidungen der Spieler resultieren. Bei den Betriebskennzahlen werden interne, das eigene Unternehmen betreffenden Kennzahlen errechnet. Unter Informationen findet man für alle Spieler geltende Stücklisten, Beschaffungskosten z.B. für Lagerhallen oder auch 
Marktforschungsergebnisse. Die Nebenbereiche gliedern sich in Graphiken, Handbuch, Periodenbericht, Data Import, Data Export und Lizenz und bilden bis auf die Graphiken $^{136}$ die Rahmenbedingungen für den Spieler dar.

Das Computerprogramm des SPIELLEITERS $\sqrt{137}$ simuliert die Märkte. Hier kann die Spielleitung Einfluss auf die Entwicklung der Nachfrage, der Preise für Produktionsgüter oder der Transportkosten nehmen.

Haben die Spieler ihre Entscheidungen getroffen, übersenden sie eine Datendatei mit ihren Entscheidungen über das Internet oder per Diskette an die Spielleitung. Die Spielleitung liest die Dateien der fünf Unternehmen in das Leitungsprogramm SPIELLEITER ein. Mit einem Algorithmus, der u.a. die Preise, Werbe-, F\&E- und sonstige Aufwendungen als Parameter verarbeitet, werden die möglichen Absatzmengen jedes Unternehmens bestimmt. Ob die mögliche Absatzmenge wirklich realisiert werden kann, hängt von der Bestandsführung im Verkaufsartikellager eines jeden Unternehmens $\mathrm{ab}$.

\footnotetext{
136 Der Nebenbereich Graphiken ist ein reiner Erklärungsbereich. Mit Abschluss jeder Periode werden automatisch Graphiken erzeugt, die sich in einem bestimmten Verzeichnis auf der Festplatte befinden.

137 Zum besseren Verständnis und für die bessere Lesbarkeit wird nicht der echte Dateiname (LPLUSMOD.xls) sondern das Wort SPIELLEITER angegeben. Näheres zum Spielleitermodul erfährt man in Kapitel 6.
} 


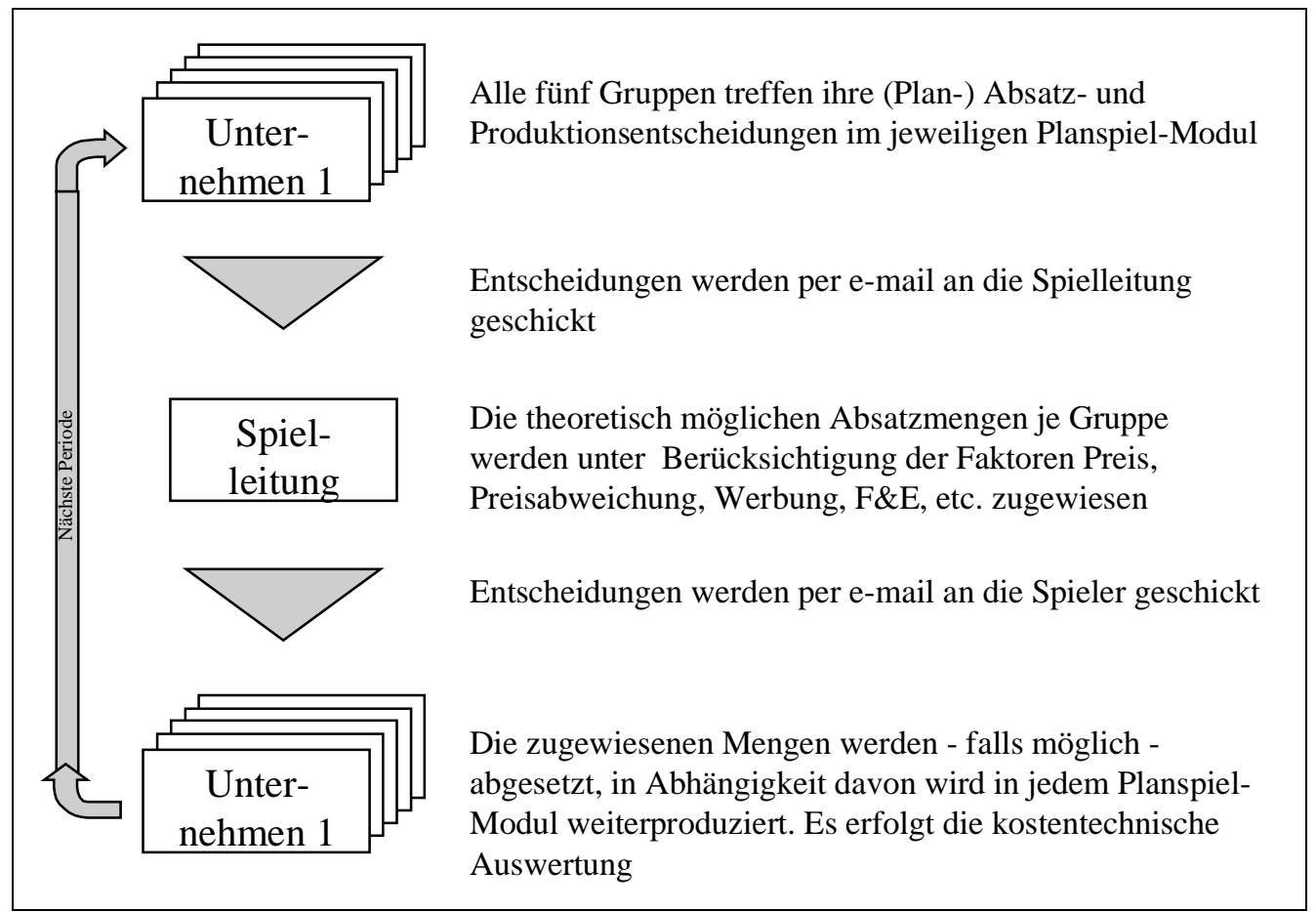

Abb. 11: Abwicklung einer Periode

Diese Art der Vorgehensweise und Definition der Funktionen der beiden Computerprogramme gewährleistet, dass die Berechnung der Ergebnisse der Unternehmungen direkt bei deren Eingabe erfolgen kann. Durch die (Vor-) Simulation kann jeder Spieler erkennen, welche Auswirkungen sich ergeben werden, wenn die getroffenen Voraussetzungen wie z.B. Preis und Absatzmenge eintreffen. Das Spielleitermodul ist somit „nur“ für die Koordination bzw. die Markt- und Absatzsimulation sowie für die Auswertung da.

\subsubsection{Das Computerprogramm für den Spieler}

Das Planspiel LogisticPLUS ist als komprimierte Dateien auf zwei Disketten oder einer CD-ROM gespeichert. Zuerst ist die Datei LPLUS-1 zu entpacken. Dies geschieht am 
besten mit dem Programm WINZIP ${ }^{138}$ Diese Datei muss in ein Verzeichnis LPLUS entpackt werden. Hierunter finden sich dann folgende Dateien und Verzeichnisse:

\section{Dateien}

1. HANDBUCH Automatischer Start des HTML-Handbuches.

2. LIESMICH Informationen zur Installation.

3. LOGPLUS Geschützte Startdatei.

4. LPLUSDAT Das ebenfalls geschützte Datenfile.

\section{Verzeichnisse}

1. DATA Daten des Spielers.

2. GRAPHICS Speicherort für automatisch erstellte Graphiken beim Einlesen der Ergebnisse einer Periode.

3. INFOS Berichte der Spielleitung. Diese können direkt aus dem Planspielmodul aufgerufen werden.

4. MANUAL Daten des Handbuches.

Die gezippte Datei LPLUS-2.zip enthält die eigentlich für den Spieler vorgesehene Planspieldatei LPLUSDAT.xls. Dieses File muss im Verzeichnis LPLUS (neben die oben erwähnten drei Dateien) abgelegt werden.

\subsection{Leistungsströme bei LogisticPLUS}

Im vorliegenden logistischen Planspiel kommt es in erster Linie auf den Leistungsbereich an. Ihn durchziehen Leistungsströme, angefangen vom Einkauf, über Produktion, Montage bis hin zum Absatz. Vereinfacht spricht man auch vom Input und Output. Das Pendant dazu ist der Finanzbereich bzw. sind die Finanzströme mit Einund Auszahlungen. Das folgende Bild veranschaulicht beide Bereiche:

\footnotetext{
138 Zippen ist ein Begriff, der für das komprimieren von Dateien steht. Die Dateien wurden in diesem Fall mit dem Programm Winzip 7.0 gezippt. Die Software ist als Testversion im Internet unter http://www.winzip.de erhältlich, es existiert ein Copyright $@$ der Niko Mak Computing, Inc. und der H. C. Top Systems B.V.
} 


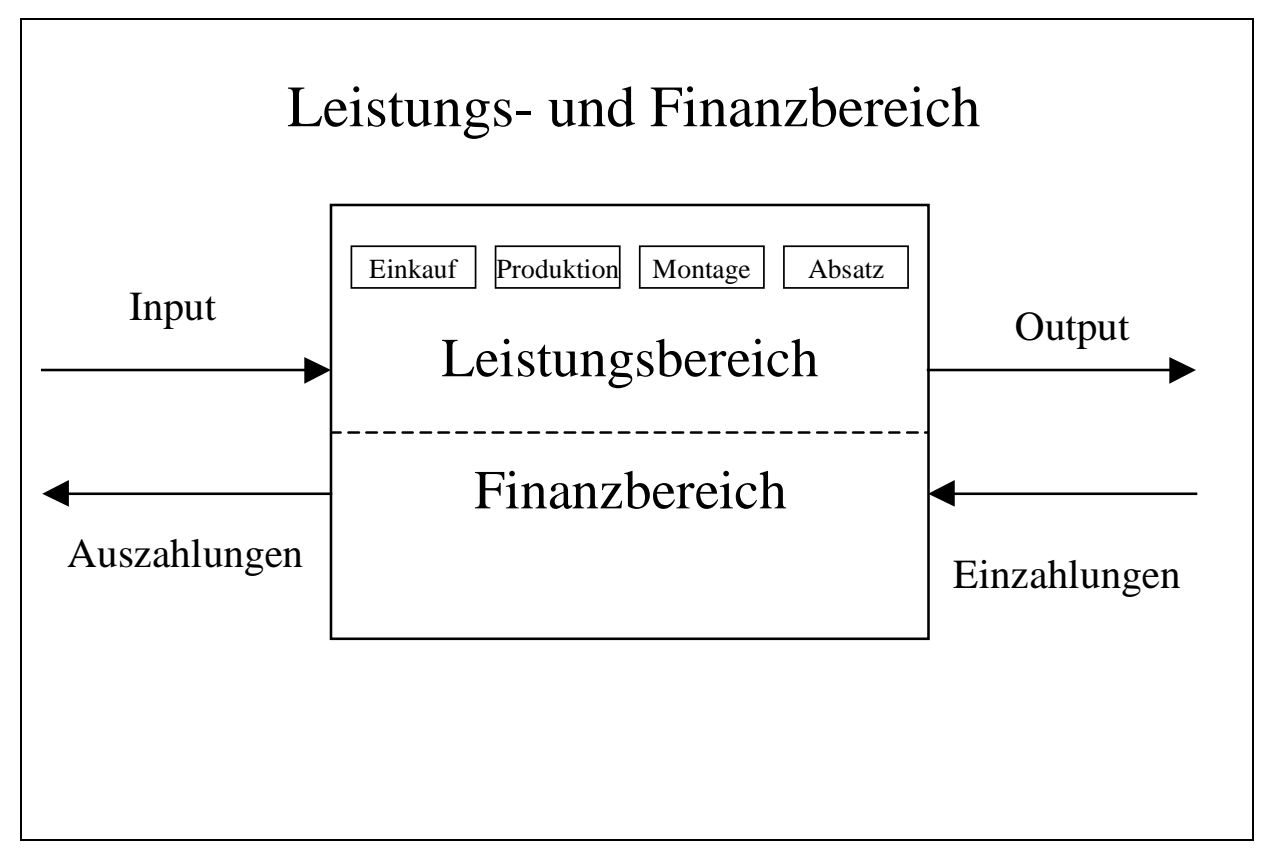

Abb. 12: Leistungs- und Finanzbereich bei LogisticPLUS

Um detaillierter in das Unternehmen und in die Logistik und Produktionsstrukturen einzusteigen, ist der Leistungsfluss (oder auch Materialfluss) genauer zu betrachten.

\footnotetext{
139 Die Startdatei ist nötig, um die Initialisierung durchzuführen. Insbesondere muss das Laufwerk ermittelt werden, in dem sich das Verzeichnis „LPLUS“ befindet.

140 Vgl. Wöhe (ABWL, 2000) Seite 617.
} 


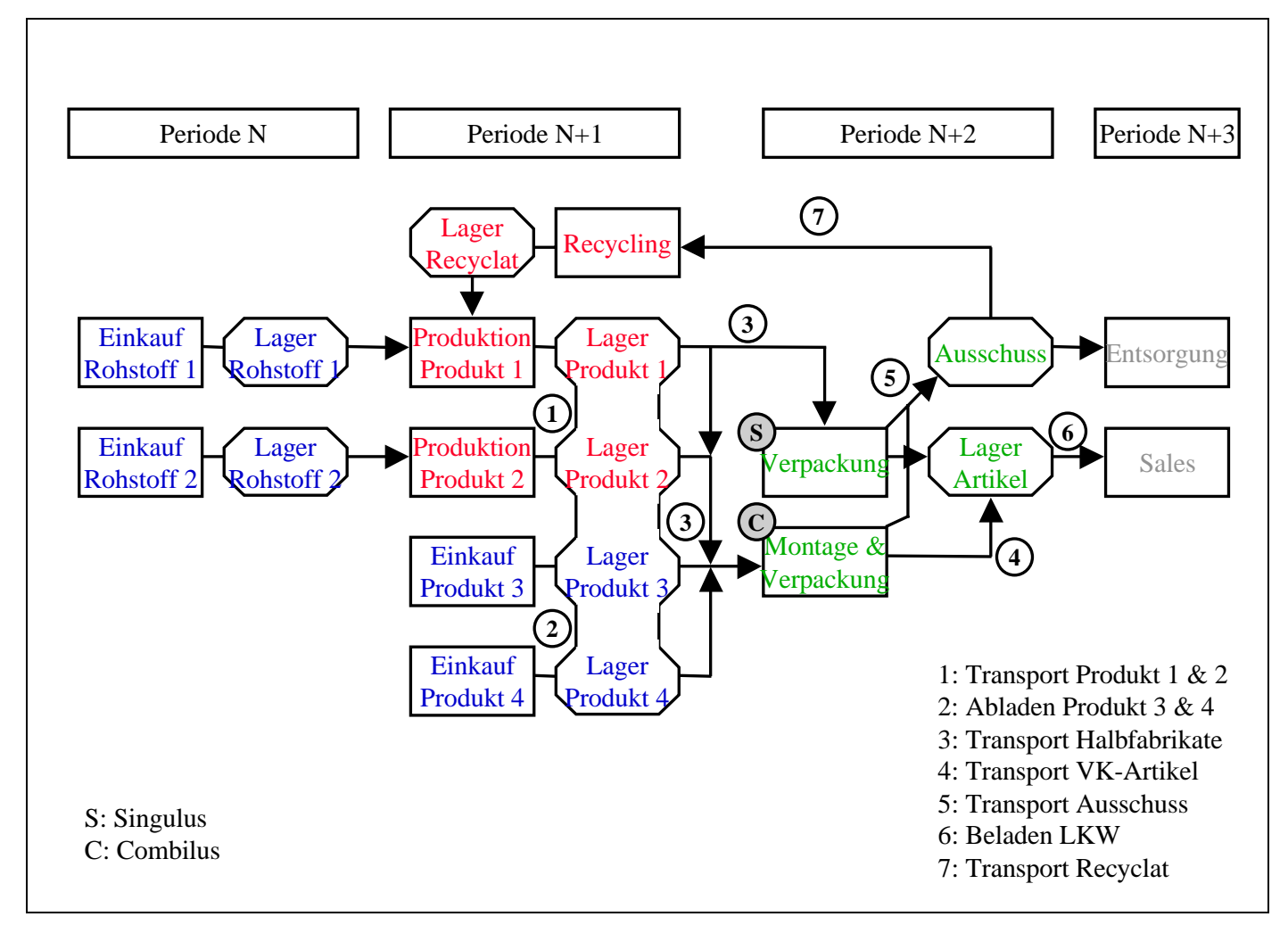

Abb. 13: Leistungsfluss LogisticPLUS

In obigem Bild ist die Mehrstufigkeit des Wertschöpfungsprozesses gut erkennbar. In der Periode $\mathrm{N}$ werden zwei Rohstoffe gekauft und eingelagert. In der folgenden Periode $\mathrm{N}+1$ werden aus diesen Rohstoffen zwei Halbfabrikate (Produkt 1 und 2) hergestellt und in das für alle Produkte verwendete Halbfabrikatlager - im Bild in der Mitte eingelagert. In dieser Periode müssen auch die zwei zugekauften Produkte 3 und 4 bestellt und eingelagert werden. In der Periode N+2 werden aus den vorhandenen vier Halbfabrikaten die zwei Verkaufsartikel Singulus und Combilus montiert und verpackt und in das Lager oder ggf. in das Ausschusslager transportiert. In der letzten Periode $(\mathrm{N}+3)$ werden die Verkaufsartikel dem Markt zugeführt oder entsorgt. Um die Komplexität etwas zu erhöhen, besteht die Möglichkeit dem Produktionsvorgang 1 Recyclat zuzuführen. Die dafür nötigen Stoffe müssen dementsprechend rechtzeitig durch Entnahme aus dem Ausschusslager eingeplant werden. 


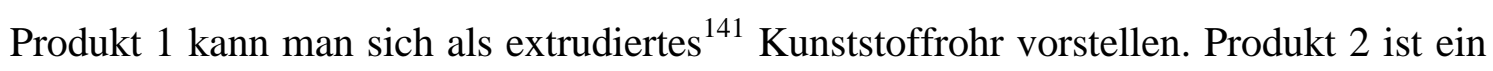
gespritztes ${ }^{142}$ Kunststoffteil, z.B. eine Tragplatte für die Montage eines Waschbeckens. Das von einer Tochtergesellschaft zugekaufte Produkt 3 ist ein bereits vormontiertes Kunststoffprodukt, z.B. ein Spülkasten. Produkt 4 sind Metallteile, die von externen Lieferanten geliefert werden.

Der VK-Artikel Singulus ist ein Ablaufrohr mit ca. $8 \mathrm{~cm}$ Durchmesser, der VK-Artikel Combilus ist ein sanitäres Installationssystem für die Konstruktion einer Vorwand im Bad.

Mittels eines Recyclingprozesses ist es möglich, die aufgrund von Qualitätsanforderungen ausgemusterten Produkte des Rohstoffes 1 wieder dem Stoffkreislauf einzubringen. Im anderen Fall ist der Ausschuss zu bestimmten Kosten zu entsorgen.

Insbesondere im Recyclingprozess wird an die Planung, Gestaltung und Logistik eine spezifische Herausforderung gestellt: Die effiziente Verbindung von Neuproduktion und Produktrecycling $\mathrm{zu}$ recyclingintegrierenden Produktionssystemen. Diese Herausforderungen lassen sich auf vier Faktoren zurückführen:

1. Aufgrund der Teilprozesse des Produktrecyclings und einer deswegen größeren Anzahl unterschiedlicher Fertigungsvorgänge ergibt sich ein komplexerer Materialfluss.

2. Die beiden zu verknüpfenden Hauptprozesse zeichnen sich durch entgegengesetzte Flussrichtung aus.

3. Die Hauptprozesse Neuproduktion und Produktrecycling sind nicht unabhängig voneinander, sondern weisen aufgrund der sie verbindenden Materialströme Wechselwirkungen auf.

141 Extrusion ist ein Produktionsvorgang, der Produkte in endloser Art herstellt. In diesem Beispiel wird ein heißes Kunststoffgemisch mittels einer sogenannten Extrusionsdüse hergestellt. Dieses heiße Rohr wird durch Wasserbäder abgekühlt und damit in eine feste Form gebracht. Mit Hilfe von Messgeräten ist eine Regelung der Dicke innerhalb eines Toleranzfeldes möglich. Das Rohr wird gekennzeichnet und automatisch abgelängt und einer Verpackung zugeführt.

142 Spritzguss (engl.: injection moulding) ist ein gussähnliches Verfahren. In diesem Beispiel wird ein heißes Kunststoffgemisch mit einem bestimmten Druck in eine Stahlform gespritzt. Mit Kühlschläuchen innerhalb dieses (Stahl-) Werkzeuges wird der Kunststoff aufgehärtet. Anschließend wird das Werkzeug hydraulisch geöffnet und das Teil automatisch abgeführt. 
4. Das Produktrecycling zeichnet sich im Vergleich zur Neuproduktion durch ein erhöhtes Unsicherheitsniveau bei der Prognose von Input-Output-Relationen aus.

Es existieren drei Absatzmärkte, die unterschiedlich ausgewertet werden: Markt Inland ist tendenziell teurer als Markt Ausland (Export). Hier findet eine ausschließliche Konkurrenz der am Planspiel beteiligten Unternehmen statt. Der Auslandsmarkt, auf dem noch weitere als die fünf teilnehmenden Unternehmen konkurrieren, ist prinzipiell geringeren Schwankungen bei der Preisbildung unterworfen. Weiterhin kann die Spielleitung gegebenenfalls bestimmte Produkte ausschreiben. Hier erhält das Unternehmen mit dem billigsten Angebot den Zuschlag. 144

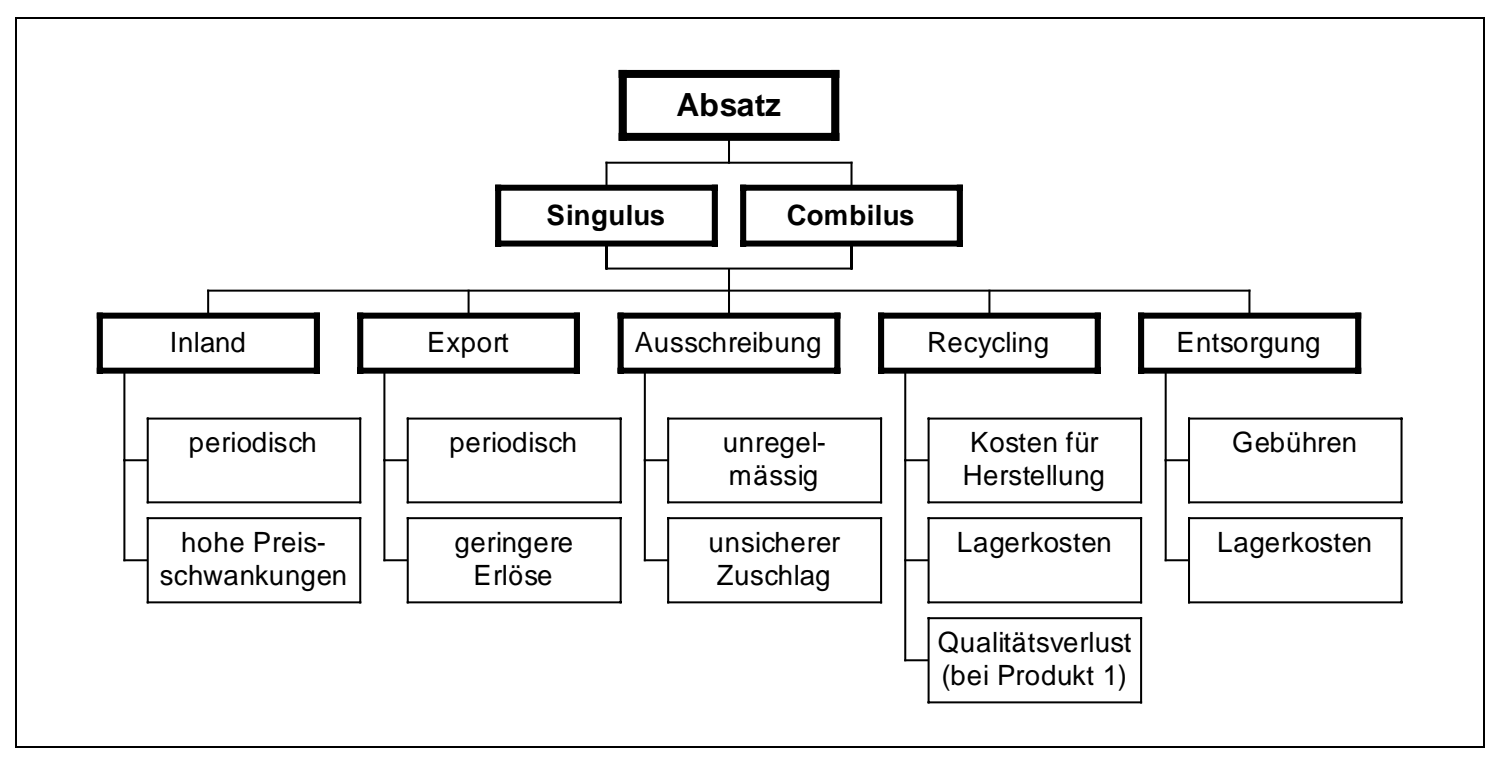

Abb. 14: Absatzstruktur LogisticPLUS

Der bereits angesprochene Recyclingprozess wird als Teilfunktion des Absatzes angesehen: (Fehl-) Produzierte Erzeugnisse des Produktes 1 können entweder wiederverwendet oder entsorgt werden, fehlerbehaftete Teile der anderen Produkte müssen gegen Gebühren entsorgt werden.

Der Finanzstrom zieht sich über die vier Funktionen des Unternehmensprozesses hinweg. Der Kassenbestand ist dazu gegenläufig: Nur aufgrund der abgesetzten

143 Vgl. dazu Schenk / Jahn (Recycling, 1997) Seite 137 f. 
Produkte kann ohne Kreditaufnahme neuer Rohstoff eingekauft bzw. Zulieferprodukte beschafft werden.

\subsection{Verschiedene Entscheidungsbereiche}

Wenn wir von Entscheidungen sprechen, sind damit nur Entschlüsse gemeint, die sich auf die Verwendung verfügbarer Mittel beziehen. In der Literatur werden solche Entschlüsse Mittelentscheidungen genannt.

Die nachfolgenden Kapitel beschreiben die einzelnen Funktionen des Planspiels. Hierbei werden auch Abhängigkeiten bzw. Folgen der einzelnen Entscheidungsvariablen definiert. Im einzelnen sind dies die Bereiche Marketing, Produktion, Logistik, Qualitätssicherung, Finanzierung und Personalwirtschaft.

\subsubsection{Absatz und Marketing}

\subsubsection{Marktforschung und Wettbewerbsberichte}

Die Spieler müssen sich einen Überblick über ihr eigenes Unternehmen, die Märkte und die Zukunftserwartungen verschaffen. Dazu stehen zwei unterschiedliche Instrumente zur Verfügung: Periodenberichte der Spielleitung und Marktforschungen.

Die Spielleitung versorgt zu Beginn einer jeden Periode die Spieler mit bestimmten (gleichen und unendgeldlichen) Informationen (Periodenberichte). Hier werden z.B. Ausschreibungen, Preisveränderungen der Rohstoffe oder Markttendenzen aufgezeigt.

Um einen Überblick über die Wettbewerber und die Märkte zu erhalten, sind Marktforschungen notwendig. Es besteht die Möglichkeit, in jeder Periode zwei

\footnotetext{
144 Bei Gleichheit mehrer Angebote wird das Ausschreibungsvolumen aufgeteilt.

145 Brede (Verwaltungsentscheidungen, 1968) Seite 13.
} 
unterschiedliche Marktforschungsberichte $\mathrm{zu}$ kaufen. Diese werden durch ein unabhängiges Institut (die Spielleitung) bereitgestellt.

Der kleine Marktforschungsbericht enthält nachfolgende Zahlen:

1. Gesamtmarktvolumen (Absatz) Singulus und Combilus, jeweils Inland und Export, jeweils mit einer Unsicherheit von 5 Prozent.

2. Summe des Gesamtumsatzes (alle Märkte) aller Wettbewerber mit einer Unsicherheit von 5 Prozent.

3. Preisdurchschnitt und Preisminimum im Inland für Singulus und Combilus.

4. Kennzahlen der Wettbewerber: Umsatz, Periodenerfolg, Gesamterfolg, Cashflow, Eigenkapitalrentabilität, Analagenintensität und Mitarbeiterzahl (Summe) sowie jeweils die Änderung gegenüber der letzten Periode. 146

Dabei ist zu beachten, dass die Daten der vergangenen Periode dargestellt werden. Die Bezeichnung der Wettbewerber in Firma A, B, C und D lässt keinen Rückschluss auf die Spielergruppe zu. 147

Wird der große Marktforschungsbericht beauftragt, werden neben dem kleinen Marktforschungsbericht zusätzlich folgende Informationen bereitgestellt:

1. Effektives Werbevolumen ${ }^{148}$ aller fünf Wettbewerber sowie des eigenen Unternehmens.

2. Preisdurchschnitt und Preisminimum im Export für Rohre und Systeme

3. Kennzahlen des Wettbewerbs: Umsatz Singulus und Umsatz Combilus, jeweils mit Markt- und Exportanteil.

Auch hier werden die relevanten Werte der Wettbewerber mit einer Unsicherheit von 5 Prozent angegeben.

146 Die Änderung gegenüber der letzten Periode wird nur angezeigt, wenn auch in der letzten Periode eine Marktforschung beauftragt wurde. Ansonsten ist das Feld leer.

147 Für jede der fünf Unternehmen wird die Zuweisung der Konkurrenten auf die Buchstaben A bis D anders vollzogen. So könnte in der Auswertung des Unternehmens 1 das Unternehmen 2 als Konkurrent A, das Unternehmen 3 als Konkurrent B, das Unternehmen 4 als Konkurrent $\mathrm{C}$ und das Unternehmen 5 als Konkurrent D dargestellt werden. Für das Unternehmen 2 könnte die Zuweisung wie folgt lauten: Konkurrent A ist Unternehmen 4, Konkurrent B ist Unternehmen 3, Konkurrent C ist Unternehmen 1 und Konkurrent D ist Unternehmen 5.

148 Das effektive Werbevolumen beschreibt die Nachhaltigkeit einer Werbemaßnahme über eine Periode hinaus. Näheres im Kapitel Absatzunterstützung. 


\subsubsection{Preispolitik und ihre Wirkung}

Die Spielleitung gibt das maximale Marktvolumen auf den unterschiedlichen Märkten mittels einer Funktion vor.

Der mögliche Anteil der einzelnen Spieler sowie das endgültige Marktvolumen werden über den Durchschnittspreis aller Spieler bestimmt (vgl. nachfolgende Abb.). Durch diese Abhängigkeit des Volumens vom Marktpreis wird erreicht, dass die Spieler die Preise der Verkaufsprodukte Singulus und Combilus an den Selbstkosten orientieren. 149

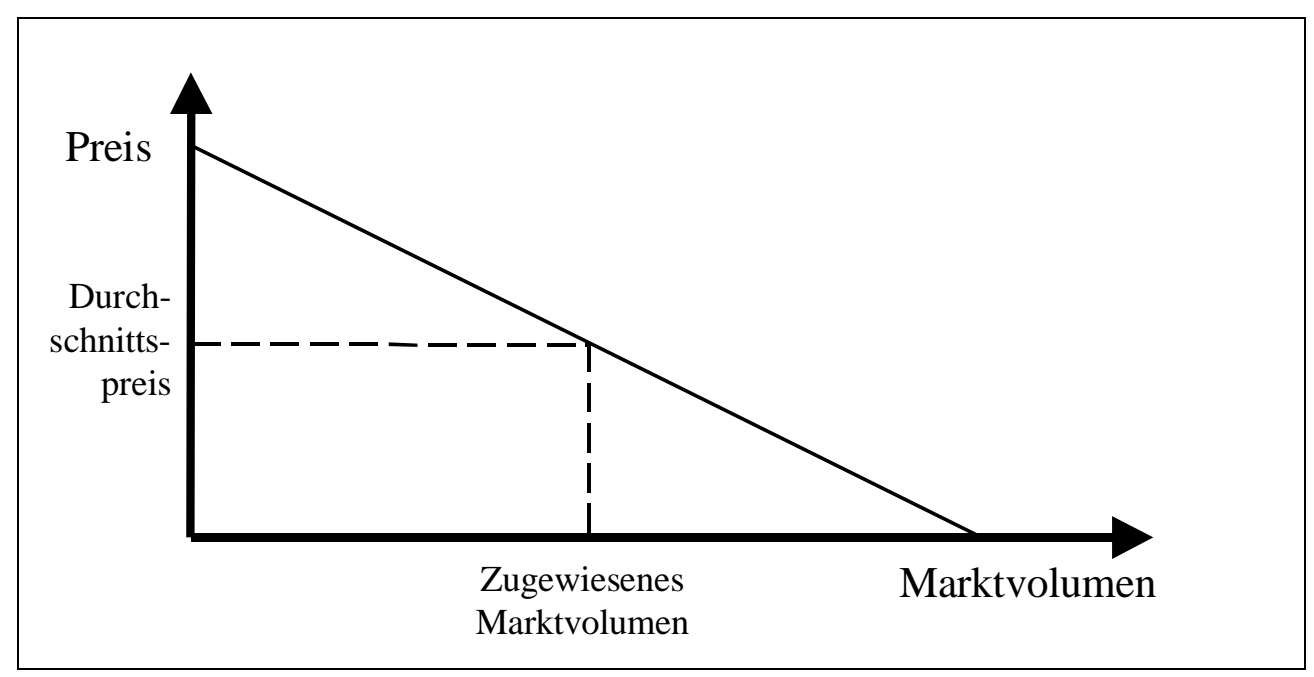

Abb. 15: Bestimmung des Marktvolumens

Eine lineare Nachfragefunktion führt vom Marktvolumen Null bis zur Mitte des Geradenabschnitts zu einer Zunahme des wertmäßigen Volumens (Preis * zugewiesenes Volumen).

Die Preisabweichung vom Durchschnittspreis bestimmt die Nachfrageunterschiede zwischen den einzelnen Unternehmen, ausgedrückt durch den Preisfaktor. Hierbei wird auch die Preisänderung seit den letzten zwei Perioden mit berücksichtigt.

149 Aufgrund von Absprachen unter den fünf teilnehmenden Spielern könnte ein sehr hohes Preisniveau erreicht werden. Dies würde bei der Parametereinstellung zu extrem hohen Gewinnen führen. 


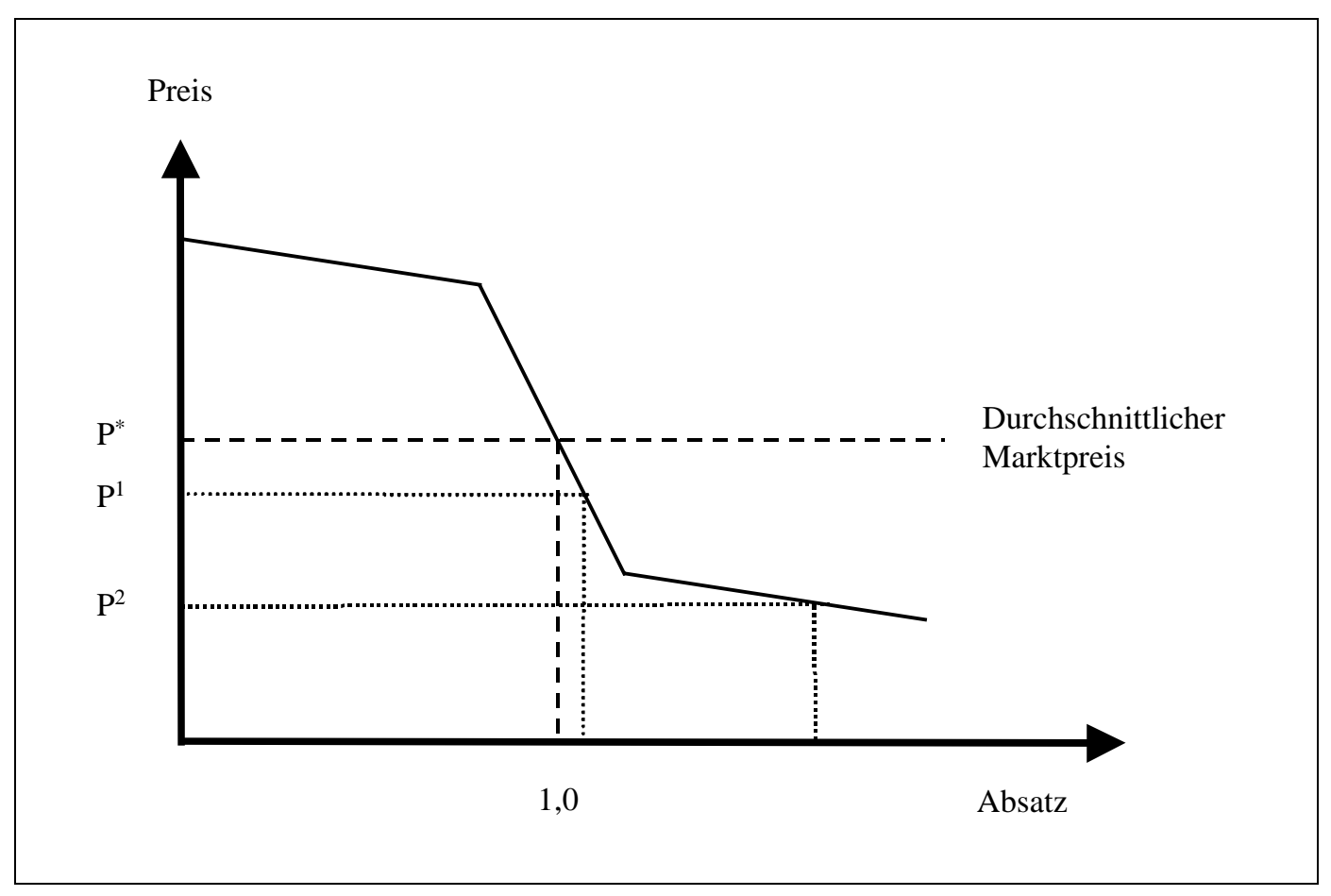

Abb. 16: Preisabweichung vom Durchschnittspreis 150

Eine geringe Abweichung des eigenen Preises $\left(\mathrm{P}^{1}\right)$ zum Marktdurchschnittspreis $\left(\mathrm{P}^{*}\right)$ hat eine geringe Auswirkung auf den Absatz. Erst ab einer bestimmten Größe wächst oder sinkt der Absatz rapide (z.B. bei $\mathrm{P}^{2}$ ).

\subsubsection{Absatzunterstützung}

Das maximale Marktvolumen wird auf den unterschiedlichen Märkten durch die Spielleitung bzw. den Durchschnittspreis vorgegeben (siehe vorhergehendes Kapitel). Der mögliche Anteil der einzelnen Spieler wird prinzipiell über den Preis bzw. die Preisabweichung bestimmt.

Es existieren jedoch außer dem Preis weitere Parameter, die die Absatzwirkung positiv oder negativ beeinflussen:

1. Werbeaufwand (Werbefaktor): Effektive Werbeausgaben und der sogenannte Werbefaktor werden im Anschluss exemplarisch beschrieben.

150 Vgl. hierzu auch Bea et al. (AWBL, 2000), Schierenbeck (BWL, 2000) Seite 224, Wöhe (ABWL, 2000) oder Woll (AVWL, 2000) 
2. Preisänderung zur Vorperiode (Preisfaktor): Bei der Vergabe des Marktpotenzials wird berücksichtigt, wie die Preisänderung eines Unternehmens im Verhältnis zu den anderen Unternehmen steht. Dies geschieht über die Bildung einer durchschnittlichen Preisänderung jedes Unternehmens über die letzten zwei Perioden. Diese Preisänderung wird verglichen mit der durchschnittlichen Preisänderung aller Unternehmen. Ähnlich der Preisabweichung vom Durchschnittspreis und der damit folgenden Änderung des möglichen Absatzvolumens wird diese Preisänderungsabweichung mit in die Zuteilung einbezogen.

3. Liefertreue (Liefertreuefaktor): Es wird überprüft, inwieweit in den letzten zwei Perioden das zugewiesene Marktpotenzial erfüllt wurde. Kann aufgrund von fehlenden Artikeln im Lager nur ein geringer Teil des zugewiesenen Marktvolumens (und damit Bestellungen von Kunden) geliefert werden, wird in der kommenden Periode weniger zugeteilt.

Alle drei Parameter, die im folgenden Unterstützungsfaktoren genannt werden, sind abhängig von den Aktionen der Konkurrenten. Beispiel Preisänderung: Erhöhen alle fünf Spieler den Preis um 10 Prozent, hebt sich die (negative, weil normalerweise Absatz mindernde) Wirkung der Preiserhöhung auf.

Mit den Werbeausgaben einer Periode wird eine Werbeagentur eine Kampagne durchführen, die das Produkt auf dem Markt den potentiellen Kunden bekannt macht (Fachzeitschriften, Mailings, etc.). Der Werbeerfolg ist nur von der Ausgabenhöhe abhängig. Es gibt keine qualitativen Unterschiede in der Werbung.

Eine einmalige Ausgabe (= Werbekampagne) hat eine nachhaltige Wirkung bei den potentiellen Kunden über drei Perioden: in der aktuellen und in den zwei Folgeperioden.

Die drei Teilbeträge aus der Werbung ergeben zusammen die wirksamen Werbeausgaben einer Periode. Die Höhe der wirksamen Werbungsausgaben bestimmt der Werbefaktor, der die Absatzmenge des eigenen Unternehmens beeinflusst. 


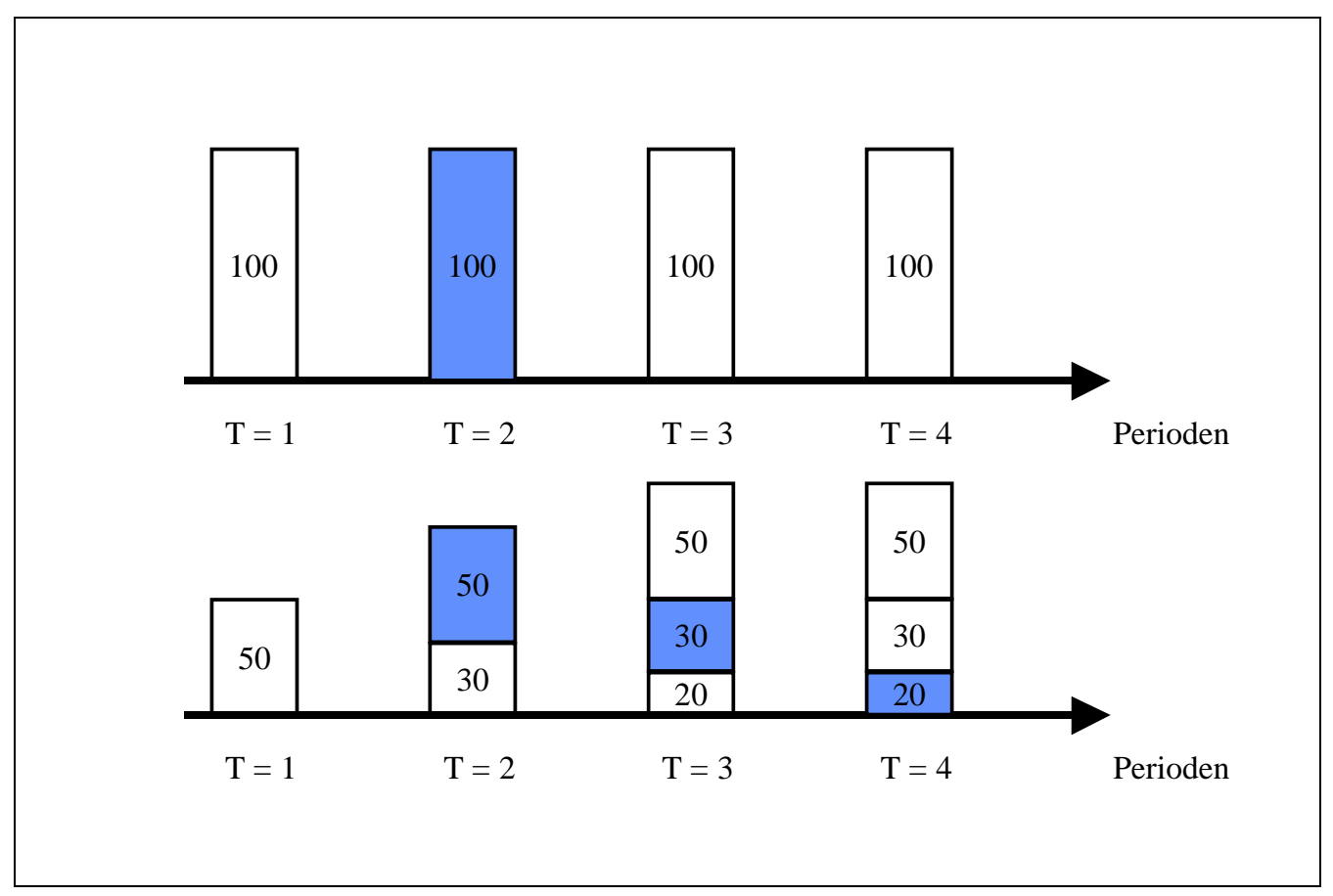

Abb. 17: Wirksamkeit der Werbung (effektive Werbung)

In obiger Abbildung wird die Werbewirkung eines Betrages, der in der Periode $\mathrm{T}=2$ eingesetzt wird, gezeigt. Die Wirkung verteilt sich auf die Perioden $\mathrm{T}=2, \mathrm{~T}=3$ und $\mathrm{T}=4$.

Der Werbefaktor ergibt rechnerisch einen Wert zwischen einer unteren und einer oberen Grenze, der bei der Ermittlung der Nachfrage berücksichtigt wird. Die Summe der Werbefaktoren aller fünf Teilnehmer ergibt in der Regel den Wert 5. Die einzelnen effektiven Werbeausgaben werden anteilmäßig aufgeteilt. 


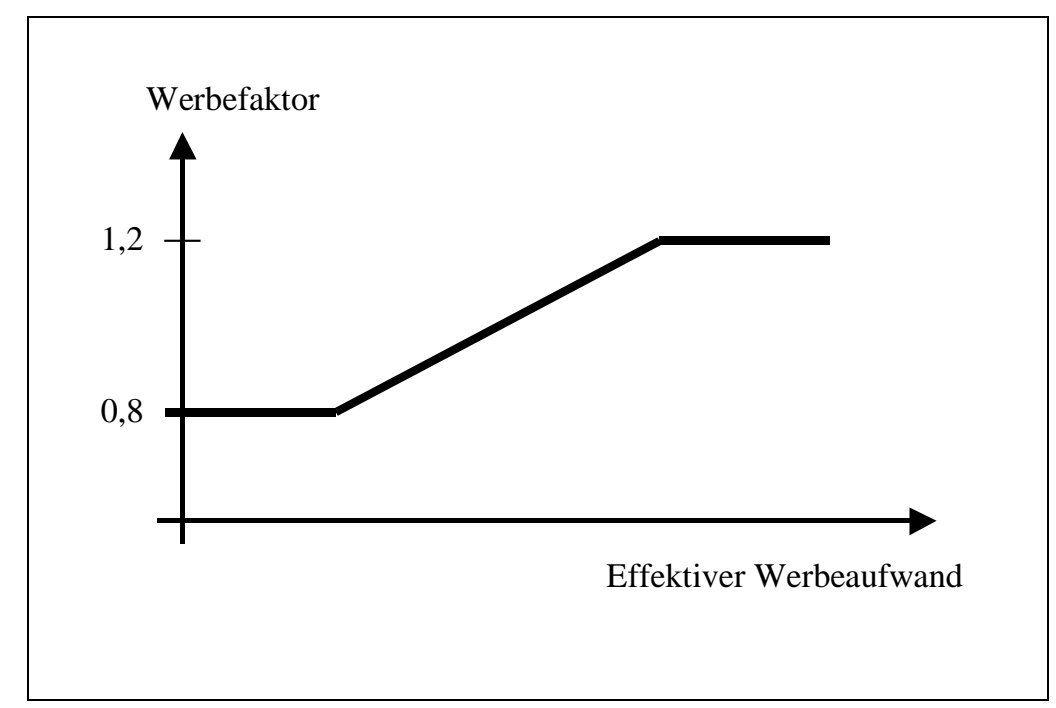

Abb. 18: Werbefaktor

Der Werbefaktor berechnet sich auch unter Einbeziehung der effektiven Werbeausgaben der vier anderen Spieler. Dadurch wird erreicht, dass der Spieler mit der größten effektiven Werbung auch den größten Einfluss auf die Marktentwicklung hat. Jedoch sind die Einflussmöglichkeiten begrenzt: Ab einem bestimmten unteren Betrag wird der Werbefaktor auf 0,8, ab einem bestimmten oberen Betrag wird der Werbefaktor auf 1,2 festgelegt. Die Berechnung erfolgt automatisch wie das folgende Beispiel hier nur für drei Unternehmen zeigt.

\begin{tabular}{|c|r|c|c|}
\hline Unternehmen & $\begin{array}{c}\text { effektive } \\
\text { Werbung }\end{array}$ & $\begin{array}{c}\text { Werbefaktor } \\
\text { rechnerisch }\end{array}$ & $\begin{array}{c}\text { Werbefaktor } \\
\text { definitiv }\end{array}$ \\
\hline 1 & $10.000 €$ & 2 & 1,2 \\
\hline 2 & $3.000 €$ & 0,6 & 0,8 \\
\hline 3 & $2.000 €$ & 0,4 & 0,8 \\
\hline Summe & $15.000 €$ & 3 & 2,8 \\
\hline Durchschnitt & $5.000 €$ & 1 & 0,93 \\
\hline
\end{tabular}

Tab. 14: Berechnung der Werbefaktoren

Man erkennt, dass ein zu hoher Werbeaufwand nicht einen entsprechenden Effekt bringt. So ist der Aufwand von Unternehmen 1 fünfmal so hoch wie der des Unternehmens 3, allerdings ist davon nicht alles wirksam. Das Marktvolumen wird aufgrund des Summenfaktors von 2,8 nicht komplett ausgeschöpft. 
Die beiden verbleibenden Faktoren (Preisfaktor und Liefertreuefaktor) werden ähnlich dem Werbefaktor errechnet. Beim Preisfaktor ist allerdings zu berücksichtigen, dass eine höhere Preisentwicklung das zugeteilte Marktvolumen negativ beeinflusst.

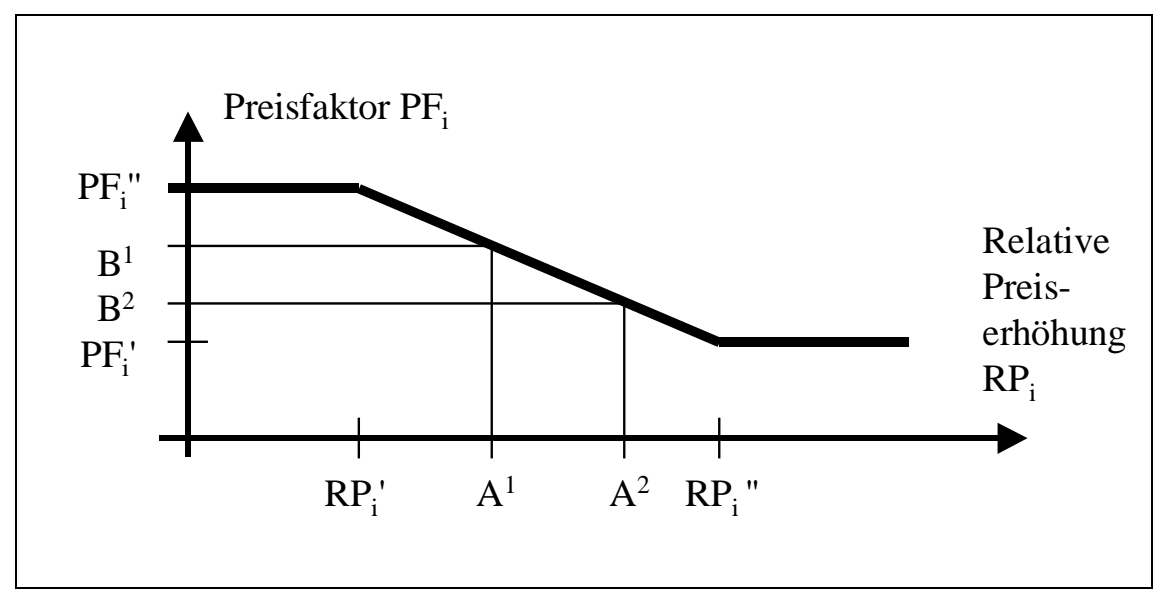

Abb. 19: Berechnung und Begrenzung des Preisfaktors

Die Einflüsse des Preisfaktors sind durch eine obere Grenze $\mathrm{PF}_{\mathrm{i}}{ }^{\text {" }}$ und eine untere Grenze $\mathrm{PF}_{\mathrm{i}}{ }^{\text {}}$ begrenzt. ${ }^{151}$ Diese sind abhängig von festgelegten Werten der relativen Preiserhöhung $\left(\mathrm{RP}_{\mathrm{i}}{ }^{\circ}\right.$ und $\left.\mathrm{RP}_{\mathrm{i}}{ }^{\circ}\right)$.Wenn die Preisabweichung eines Unternehmens bei $\mathrm{A}^{1}$ liegt, die durchschnittliche Preisabweisung aller Unternehmen bei $\mathrm{A}^{2}$, dann hat das Unternehmen geringere Preissteigerungen (oder höhere Preissenkungen) durchgeführt. Dies wird durch den höheren Preisfaktor $\mathrm{B}^{1}$ ausgedrückt.

\subsubsection{Das Produktionssystem im Planspiel}

Die Produktion besteht aus der Fertigung von zwei Halbfabrikaten (Produkt 1 und 2) und der Montage der zwei Verkaufsartikel Singulus und Combilus. Dabei werden für die zwei Halbfabrikate unterschiedliche Produktionsmaschinen, für die Montage aber nur ein (Montage-) Maschinentyp benötigt.

151 Vgl. hierzu z.B. Günther / Kruschwitz (Unternehmungsplanspiel, 1975) Seite 42 ff. 


\subsubsection{Fertigung und Fertigungssteuerung}

Bei der Fertigung werden im eigenen Unternehmen zwei Kunststoffprodukte hergestellt. Produkt 1 ist ein extrudiertes Rohr, Produkt 2 ein gespritztes Kunststoffteil (Tragplatte). Das Produkt 3 ist ein zugekauftes Halberzeugnis (Spülkasten), das von einem verbundenen Unternehmen frei Haus geliefert wird. Produkt 4 sind Metallteile, die für die Montage des Endproduktes benötigt werden.

\begin{tabular}{|l|c|c|}
\hline & Rohstoff & Volumen \\
\hline Produkt 1 (Rohr) & $0,85 \mathrm{~kg} / \mathrm{m}$ & $10 \mathrm{dm}^{3} / \mathrm{m}$ \\
\hline Produkt 2 (Tragplatte) & $1,5 \mathrm{~kg} / \mathrm{St}$ & $2,45 \mathrm{dm}^{3} / \mathrm{St}$ \\
\hline
\end{tabular}

Tab. 15: Rohstoffeinsatz und Volumen der Produkte 1 und 2

Diese Festlegung der zu produzierenden Mengen hat eine direkte Auswirkungen auf die beiden Rohstofflager: Beispielsweise sind bei einer Fertigung von 2.000 Meter Rohr 1,7 Tonnen Rohstoff nötig. Die produzierte Menge wird dann dem Halbfabrikat-Lager zugeführt und in der nächsten Periode montiert bzw. verpackt. Dem Rohstofflager (Silo) wird das entsprechende Volumen entnommen.

Für die Herstellung der beiden Halbfabrikate werden Produktionsmaschinen benötigt. Die Maschinen brauchen für jedes Produkt eine bestimmte Zeitdauer.

\begin{tabular}{|l|c|c|}
\hline & Prozess & Produktionsdauer \\
\hline Produkt 1 (Rohr) & Extrusion & $1,2 \mathrm{~min} / \mathrm{m}$ \\
\hline Produkt 2 (Tragplatte) & Spritzguss & $2,5 \mathrm{~min} / \mathrm{St}$. \\
\hline
\end{tabular}

Tab. 16: Produktionsdauer Fertigung Produkte 1 und 2

Die theoretische Gesamtkapazität der Maschinen beträgt pro Mona 20 Tage $* 24$ Stunden $=480$ Stunden. Dadurch errechnet sich die Gesamtproduktionskapazität je Maschine und Monat wie folgt:

152 Hierin sind keine Stillstandszeiten z.B. für Wartungen enthalten. Diese richten sich nach dem betriebenen Wartungsaufwand. 


\begin{tabular}{|l|c|c|}
\hline & Prozess & Produktionskapazität \\
\hline Produkt 1 (Rohr) & Extrusion & $24.000 \mathrm{~m}$ \\
\hline Produkt 2 (Tragplatte) & Spritzguss & $11.520 \mathrm{St}$. \\
\hline
\end{tabular}

Tab. 17: Gesamtproduktivität je Maschine und Monat

\subsubsection{Die Montage der Verkaufsartikel}

Wie oben beschrieben können zwei unterschiedliche Artikel abgesetzt werden: Singulus (z.B. Rohrleitungen) oder Combilus (z.B. Installationssysteme).

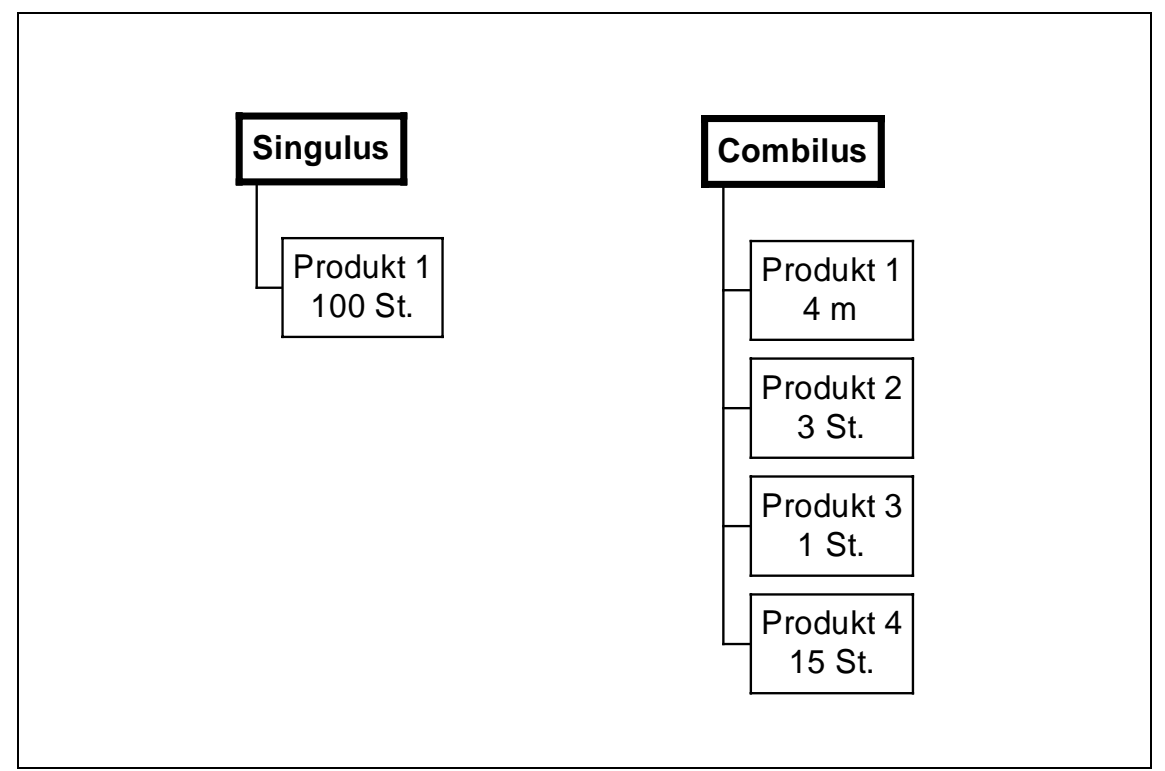

Abb. 20: Stücklisten für die VK-Artikel Singulus und Combilus

Singulus wird in Kubikmetern verpackt. Dies geschieht mit Montage- bzw. Verpackungsautomaten. Ein Verkaufsartikel besteht aus 100 Meter Rohr, der auf einer Palette verpackt wird. Das Volumen, das für die Verladung auf den Lkw das entscheidende Maß ist, errechnet sich aus (Breite) * (Höhe) * (Länge) zu $(10$ Rohre $* 10 \mathrm{~cm}) *(10$ Rohre $* 10 \mathrm{~cm}) * 1 \mathrm{~m}=1$ Kubikmeter. Die 
Kommissionierungsdauer je einem Meter Rohr beträgt 1,2 Sekunden, d.h. für einen VKArtikel benötigt man 2 Minuten.

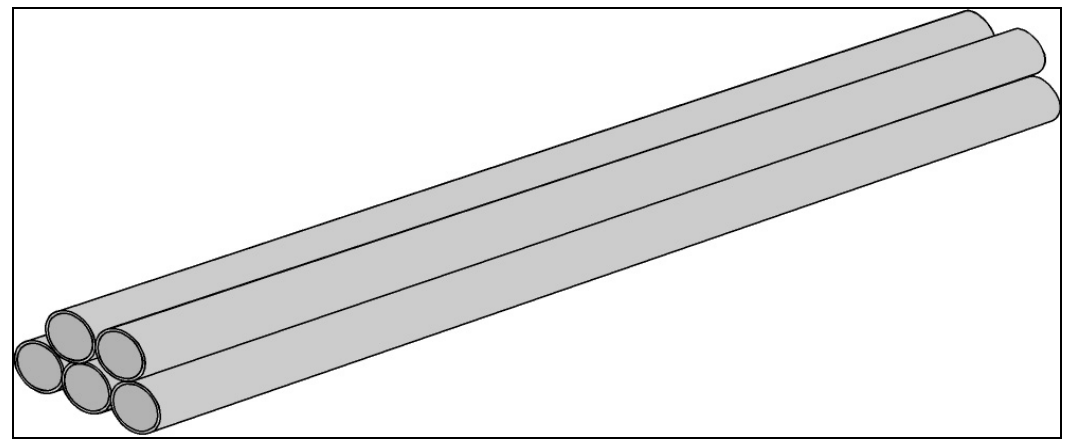

Abb. 21: Verkaufsartikel Singulus

Bei der Montage von Combilus werden die vier unterschiedlichen Produkte zu einem Endprodukt zusammengesetzt bzw. zu einer Charge zusammengestellt. Dies geschieht manuell mit Unterstützung derselben Montage- bzw. Verpackungsautomaten. Es ist festzulegen, wie viele Endprodukte zusammengesetzt werden sollen. Nachfolgend die komplette Stückliste:

\begin{tabular}{|l|r|r|c|c|}
\cline { 2 - 5 } \multicolumn{1}{c|}{} & \multicolumn{1}{c|}{ Gewicht } & \multicolumn{1}{c|}{ Volumen } & Montagedauer & Stückliste \\
\hline Produkt 1 (Rohr) & $0,85[\mathrm{~kg} / \mathrm{m}]$ & $10\left[\mathrm{dm}^{3} / \mathrm{m}\right]$ & & $4 \mathrm{~m}$ \\
\hline Produkt 2 (Tragplatte) & $1,5[\mathrm{~kg} / \mathrm{St}]$ & $2,475\left[\mathrm{dm}^{3} / \mathrm{St}\right]$ & & $3 \mathrm{St}$ \\
\hline Produkt 3 (Spülkasten) & $3[\mathrm{~kg} / \mathrm{St}]$ & $36\left[\mathrm{dm}^{3} / \mathrm{St}\right]$ & & $1 \mathrm{St}$ \\
\hline Produkt 4 (Metallteile) & $0,08[\mathrm{~kg} / \mathrm{St}]$ & $0,25\left[\mathrm{dm}^{3} / \mathrm{St}\right]$ & & $15 \mathrm{St}$ \\
\hline Summe (= Combilus) & $11,54[\mathrm{~kg} / \mathrm{St}]$ & $87,175[\mathrm{dm} 3 / \mathrm{St}]$ & $6[\mathrm{~min} / \mathrm{St}]$ & \\
\hline
\end{tabular}

Tab. 18: Stückliste für den VK-Artikel Combilus

153 Das Volumen ist für die Verladung auf den LKW das entscheidende Maß. Berechnung Montage- bzw. Kommissionierdauer: $100 \mathrm{~m} * 0,00033 \mathrm{Cap} / \mathrm{m}$. 


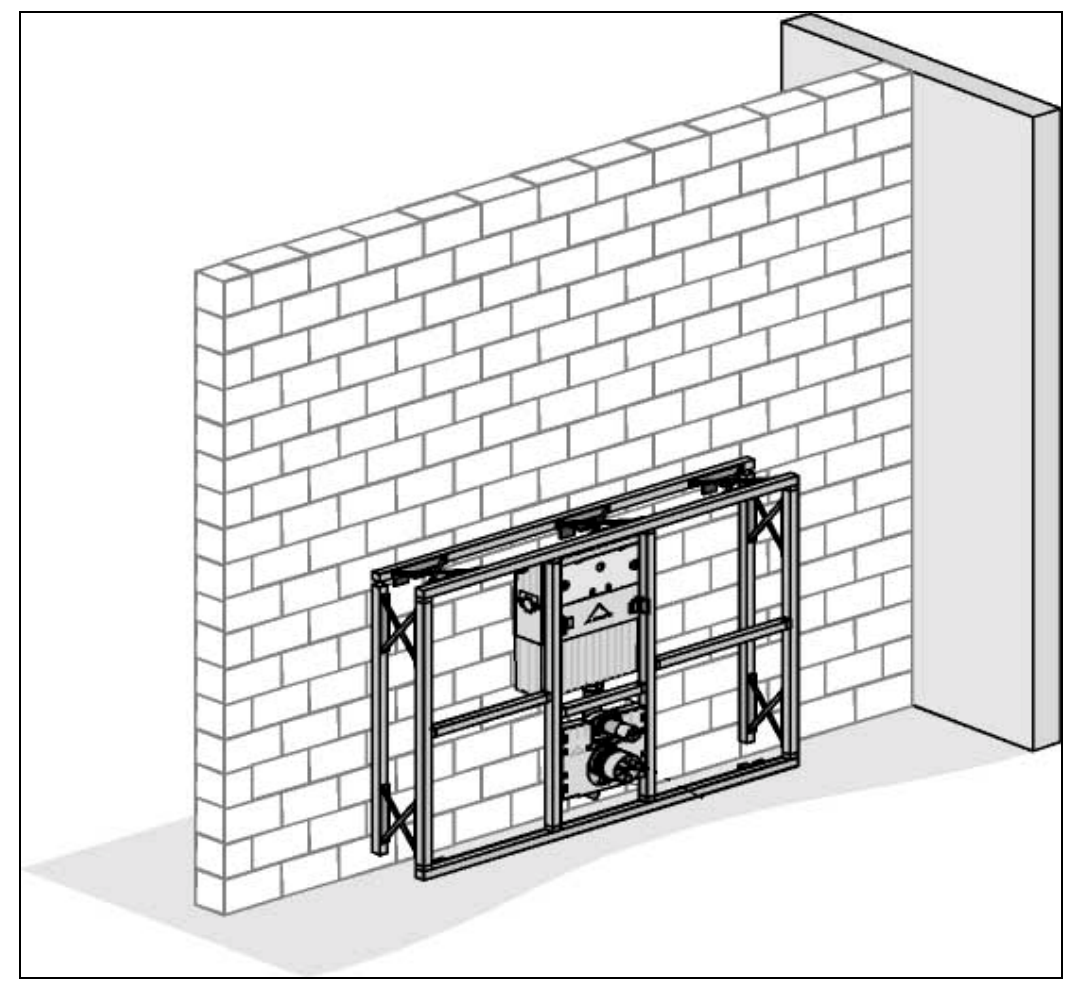

Abb. 22: Verkaufsartikel Combilus

Sehr gut erkennbar sind die Tragplatte im unteren Bereich (Produkt 2), der Spülkasten mit dem kleinen Dreieck auf der Vorderseite (Produkt 3) und die verschiedenen Metallteile des Rahmens (Produkt 4). Das notwendige Rohr (Produkt 1) fehlt in diesem Bild.

Beide Produkte sind nach der Montage versandfertig. Sie müssen nicht mehr extra verpackt werden, sondern können direkt auf LKW geladen werden. Kosten für Verpackungen oder Kosten für den Verpackungsvorgang im Sinne von Vertriebskosten fallen nicht an. 154

\subsubsection{Recycling ausgesonderter Verkaufsartikel}

Aufgrund der Beschaffenheit des Produktes 1 besteht die Möglichkeit, dem Fertigungsprozess Recyclat beizufügen. Der Recyclatanteil kann maximal 20 Prozent betragen. 
Recyclat wird durch einen externen Dienstleister aus dem vorhandenen Ausschuss hergestellt. Dafür entfallen rein variable Kosten an, es wird keine Maschine benötigt. Für das nötige Lager ist allerdings zu sorgen. Auch ist das zu recycelnde Material dem Lieferanten, der sich in unmittelbarer Nähe der Produktionsanlagen befindet, mit Gabelstaplern zu liefern. Der Lieferant kümmert sich um den Transport des Recyclats in das dafür vorgesehene Silo.

\subsubsection{Die Logistik und ihre Komponenten}

Im vorliegenden Planspiel wird - wie erwähnt - ein Schwerpunkt auf logistische Fragestellungen gelegt. Dies zeigt sich einerseits in der Anzahl der Entscheidungsvariablen, andererseits in der Vielzahl der Auswertungen auf diesem Bereich.

Die Logistik wird im Planspiel in die Bereiche Beschaffungs-, Produktions-, Distributions- und Entsorgungslogistik aufgeteilt.

\subsubsection{Beschaffungslogistik}

Die zentrale dispositive Aufgabe innerhalb der Materialbedarfsplanung besteht in der Bestimmung wirtschaftlicher Losgrößen bzw. Bestellmengen.

Für die Produktion müssen Sachgüter beschafft werden. Der gelieferte Rohstoff wird in einer bestimmten Qualität angeliefert. Durch die Beigabe von recyceltem Material wird die Qualität herabgesetzt. Dies äußert sich in einer erhöhten Ausschussquote.

Für die Rohstofflagerung stehen für jeden Rohstoff unterschiedliche Silos zur Verfügung. Insgesamt können je Rohstoff bis zu sechs Silos beschafft bzw. benutzt

\footnotetext{
154 Vgl. Wundrack (Vertriebskostenrechnung, 2000) Seite 23.

155 Die entstehenden Kosten werden im Planspiel im Bereich „Marktinformationen“ durch die Spielleitung periodisch festgelegt.
} 
werden. Ist mehr als ein Silo mit einem Rohstoff gefüllt, werden die jeweiligen Silos kombiniert betrachtet: Sie werden als ein Lager bewertet und mit der FIFO-Methode 157 befüllt bzw. entleert. Silos werden über zehn Jahre linear abgeschrieben.

Die Zukaufprodukte 3 und 4 müssen am Ende jeder Periode bezahlt werden. Pro Bestellung gelten folgende stückzahlabhängigen Preise, die gegebenenfalls durch die Spielleitung beeinflusst werden können. Hier ist zu beachten, dass das HalbfabrikatLager die Bestellung bzw. definitive Übernahme der Zukaufprodukte limitiert. Sollte das Lagervolumen nicht ausreichen, werden die Produktion der Produkte 1 und 2 sowie die tatsächliche Annahme der Zukaufprodukte 3 und 4 um den entsprechenden Überhang gekürzt.

Für die Lagerung der Halbfabrikate, der Verkaufsartikel, des Recyclats und des Ausschusses sind spezielle Lager notwendig. Diese muss man bei Bedarf neu beschaffen bzw. einrichten. Im Gegensatz zu Produktions- und Montagemaschinen können Lager nicht verkauft, sondern gegebenenfalls nur vermietet und über 50 Jahre linear abgeschrieben werden.

Falls die bestellten Rohstoffe und Zukaufprodukte wegen fehlender Lagerkapazitäten nicht angenommen werden können, sind Anfahrts- bzw. Rücknahmegebühren zu berechnen. Diese Gebühren werden in der Gewinn- und Verlustrechnung unter „Sonstige vertriebliche Aufwendungen“ und in der Kostenrechnung unter „Gemeinkosten für Reklamationen und Anfahrtsgebühren“ gebucht. Eine Rechnungsstellung des Lieferanten erfolgt jedoch nur bei der Einlagerung des Rohstoffes bzw. des Zukaufproduktes.

156 Vgl. Goertzen (Simultanplanung, 1992) Seite 1.

157 Die FIFO-Methode (first in - first out) unterstellt, dass die zeitlich zuerst beschafften Waren oder Bestände als erste verbraucht oder verkauft werden. Vgl. Bea et al. (ABWL, 2000) Seite 369. 


\begin{tabular}{|l|r|c|}
\cline { 2 - 3 } \multicolumn{1}{c|}{} & \multicolumn{1}{c|}{ Wert } & Anfahrt \\
\hline Rohstoff 1 & $1,4[€ / \mathrm{kg}]$ & $0,2[€ / \mathrm{kg}]$ \\
\hline Rohstoff 2 & $6,3[€ / \mathrm{kg}]$ & $0,1[€ / \mathrm{kg}]$ \\
\hline Produkt 3 & $14,8[€ / \mathrm{kg}]$ & $0,3[€ / \mathrm{kg}]$ \\
\hline Produkt 4 & $0,3[€ / \mathrm{kg}]$ & $0,1[€ / \mathrm{kg}]$ \\
\hline
\end{tabular}

Tab. 19: Einkaufswert und Anfahrtsgebühren für Rohstoffe und Zukaufprodukte

Für alle obigen Vorgänge gilt, dass sie nur insoweit ausgeführt werden, sofern auch die nötigen Kapazitäten zur Verfügung stehen. Sind zuwenig Kapazitäten vorhanden, werden die Aufträge soweit wie erforderlich gekürzt. Dies gilt insbesondere bei kombinierten Lagern wie dem Halbfabrikate- oder dem Verkaufsartikellager. In nachfolgender Tabelle wird die sich ergebende Reduktion bei einem fiktiven Volumen des Halbfabrikatlagers von $12.000 \mathrm{~m}^{3}$ dargestellt.

\begin{tabular}{|l|r|r|r|r|r|}
\hline & Anzahl & Volumen & Reduktion & Einlagerung & \multicolumn{1}{c|}{ Anzahl } \\
\hline Produkt 1 & $1.500 .000 \mathrm{~m}$ & $15.000 \mathrm{~m}^{3}$ & & $10.989 \mathrm{~m}^{3}$ & $1.098 .900 \mathrm{~m}$ \\
\hline Produkt 2 & $34.500 \mathrm{St}$ & $85,39 \mathrm{~m}^{3}$ & & $62,56 \mathrm{~m}^{3}$ & $25.276 \mathrm{St}$ \\
\hline Produkt 3 & $20.000 \mathrm{St}$ & $720 \mathrm{~m}^{3}$ & \multirow{2}{*}{$-27,3 \%$} & $527,47 \mathrm{~m}^{3}$ & $14.651 \mathrm{St}$ \\
\cline { 1 - 3 } \cline { 5 - 6 } Produkt 4 & $2.300 .000 \mathrm{St}$ & $575 \mathrm{~m}^{3}$ & & $421,25 \mathrm{~m}^{3}$ & $1.685 .000 \mathrm{St}$ \\
\hline Summe & & $16.380 \mathrm{~m}^{3}$ & & $12.000 \mathrm{~m}^{3}$ & \\
\hline
\end{tabular}

Tab. 20: Beispiel Volumens- und Stückzahlreduktion bei der Einlagerung

Für die Produktion braucht man unterschiedliche Produktionsgüter: Produktionsmaschinen Typ 1 (geeignet z.B. für die Extrusion von Rohren), Produktionsmaschinen Typ 2 (geeignet z.B. für das Spritzgießen von Tragplatten ) und Montage-/Verpackungsmaschinen. Maschinen können (und müssen vermutlich) neu beschafft werden. Hierbei verhilft eine Kreditaufnahme zu zusätzlichen Geldmitteln. Die Maschinen werden über 60 Perioden abgeschrieben, dies entspricht bei einer Periodenlänge von zwei Monaten einer Lebensdauer von 10 Jahren. Abgeschriebene Maschinen können nicht mehr benutzt werden. Die Entsorgung geschieht kostenfrei. 


\subsubsection{Produktionslogistik}

Aufgrund der unterschiedlichen Lagerstätten müssen Transporte unter Einsatz von Gabelstaplern, Fahrern und Hilfsarbeitern innerhalb des Unternehmens erfolgen. Die Tabelle zeigt die Anzahl der benötigten Gabelstapler, Fahrer und Hilfsarbeiter, um eine Tonne Material zu bewegen.

\begin{tabular}{|c|l|l|l|c|}
\cline { 2 - 5 } \multicolumn{1}{c|}{} & \multicolumn{1}{c|}{ Tätigkeit } & \multicolumn{1}{c|}{ von } & $\begin{array}{c}\text { Ziel } \\
\text { benötigte } \\
\text { Kapazität } \\
\text { Gabelstapler }\end{array}$ \\
\hline 1 & Transport Produkt 1 und 2 & Produktion & Lager Halbfabrikate & 0,09 \\
\hline 2 & Abladung Produkt 3 und 4 & Anlieferung & Lager Halbfabrikate & 0,08 \\
\hline 3 & Transport Produkt 1 bis 4 & Lager Halbfabrikate & Montage & 0,06 \\
\hline 4 & Transport VK-Artikel & Montage & Lager Artikel & 0,07 \\
\hline 5 & Transport Ausschuss & Montage & Ausschusslager & 0,07 \\
\hline 6 & Abfrachtung Artikel & Lager Artikel & LKW & 0,07 \\
\hline 7 & Transport Recyclat & Ausschusslager & Recycling & 0,10 \\
\hline
\end{tabular}

Tab. 21: Benötigte Gabelstapler-Kapazitäten für innerbetriebliche Transporte

Anmerkung: Die benötigten Kapazitäten sind eine Maßzahl für die Entfernungen der einzelnen Standorte bzw. die Dauer des Transportes. So benötigt z.B. ein Gabelstapler von der Produktionsanlage der Produkte 1 und 2 zum Halbfabrikatlager (Transport Nummer 1) 2,7 Minuten einfach. Da der Gabelstapler wieder zurück muss, ergibt sich als benötigte Kapazität $\frac{2 * 2.7 \mathrm{~min}}{60 \mathrm{~min}}=0,09$ Cap. Dabei sind nötige Auf- und Abladevorgänge im Zeitrahmen enthalten.

Die Rohstoffe müssen nicht mittels Gabelstaplern transportiert werden. Die Materialversorgung der Fertigung geschieht über ein vollautomatisches Rohrsystem. Dabei herrschen keine Beschränkungen bezüglich Transportmengen und -zeiten.

Der Spieler startet mit einem bestimmten Kontingent an Gabelstaplern und kann bei Bedarf Stapler zukaufen. Diese stehen dann in der folgenden Periode zur Verfügung. Weiterhin besteht die Möglichkeit, Gabelstapler für die aktuelle Periode anzumieten 
oder zu vermieten. Gabelstapler können nicht verkauft werden. Ihre Lebensdauer ist unbegrenzt, die Abschreibungen erfolgen nach der Anzahl der Gabelstapler.

Die Transportkosten setzen sich zusammen aus Abschreibungen für die Gabelstapler, Hilfs- und Betriebsstoffe sowie aus Personalkosten für Fahrer bzw. Hilfsarbeiter. Dabei werden die Kosten in fixe und variable Kosten sowie einen Zuschlagssatz bei Inanspruchnahme von Überstunden unterteilt. Überstunden bzw. Mehrkosten fallen an, wenn die in einer Periode zur Verfügung stehende Kapazität an Gabelstaplern bzw. Hilfsarbeitern überschritten wird.

Die Ursache dafür, dass ein Zuschlag eingeführt wird, ist der Umstand, dass die Produktion im Falle von Unterkapazitäten nicht „stehen bleiben“ soll. Der Spieler soll die Möglichkeit haben, seine Produktion bzw. Montageaktivitäten, wenn auch zu höheren Kosten, durchzuführen. Dieselbe Überlegung gilt auch für den Einsatz bzw. die Kostenbestimmung der Mitarbeiter.

\subsubsection{Distributionslogistik}

Die Distribution der beiden Verkaufsartikel Singulus und Combilus erfolgt über den Straßenverkehr und damit über (externe) Speditionen. Die Auswahl des Transportmittels LKW wurde gewählt, weil der Straßenverkehr weiterhin der dominierende Verkehrsträger ist.

Es muss festgelegt werden, wie viele Lastkraftwagen in jeder Periode zur Verteilung der Fertig- und Ersatzteilprodukte benötigt werden. Die Kapazität eines LKW (Anzahl der Paletten) und die damit verbundenen Kosten pro Periode ergeben sich aus der folgenden Tabelle:

158 Im Regelfall sind somit die benötigten Kapazitäten Gabelstapler gleich der benötigten Kapazitäten Hilfsarbeiter.

159 FAZ (19. September 2000): Verkehrsträger 1999: 80,6 \% Straßenverkehr, 6,8 \% Einsenbahnverkehr, 5,4\% Binnenschifffahrt, 5,1 \% Seeverkehr, 2,1 \% Rohrleitungen (Rohöl), 0,05 \% Luftverkehr. Gesamtvolumen 1999: 4.253,2 Mio. Tonnen. 


\begin{tabular}{|c|c|c|c|c|}
\hline & & Inland & Export & Ausschreibung \\
\hline Volumen & {$\left[\mathrm{m}^{3} / \mathrm{LKW}\right]$} & & je 120 & \\
\hline Bestellkosten & {$[€ / L K W]$} & & je 40 & \\
\hline Transportkosten & {$\left[€ / \mathrm{m}^{3}\right]$} & 1,00 & 2,00 & 1,00 \\
\hline
\end{tabular}

Tab. 22: Kosten für Absatztransporte

Die LKW verursachen neben den variablen und damit entfernungsabhängigen Transportkosten auch fixe Bestellkosten. Somit wird vermieden, dass der Spieler wahllos und ohne Rücksichtnahme LKW bestellt. Die bestellten LKW limitieren die Auslieferung und damit den zu erzielenden Umsatz. Dabei wird der (Plan-) Absatz je nach Inland-, Export- oder Ausschreibungsgeschäft gegebenenfalls anteilig reduziert.

Die unmittelbar aus der Absatzbeschränkung entstehenden Auswirkungen sind sehr schnell nachvollziehbar: Erstens stimmen die angenommenen finanziellen Ergebnisse nicht mehr, falls gegenüber den Planwerten nicht genügend Produkte abgesetzt werden. Das Unternehmen hat wahrscheinlich nicht genügend liquide Mittel, um die auf jeden Fall anfallenden Fixkosten und die zum Großteil anfallenden variablen Kosten zu finanzieren. Zweitens kann das VK-Artikellager nicht umgeschlagen werden. Dies bedingt vermutlich, dass die geplanten Montagemengen nicht durchgeführt werden, da das der Montage nachfolgende Lager nicht aufnahmefähig ist.

\subsubsection{Entsorgungslogistik}

Aufgrund der gewählten Struktur werden Produkte mit Fehlern erst bei der (End-) Montage erkannt. Die resultierenden Ausschussmengen der beiden VK-Artikel Singulus und Combilus werden jeweils in das Ausschusslager transportiert. Fehlerbehaftete VKArtikel vom Typ Singulus können durch den Recycling-Prozess wieder in den Produktionskreislauf zurückgeführt werden. Das restliche Material vom Typ Singulus sowie alle Ausschussmengen vom Typ Combilus müssen gegen anfallende Gebühren entsorgt werden. Dabei werden in der Bilanz Rückstellungen in Höhe der zu erwartenden Kosten gebildet. 
Die Entsorgungslogistik bezieht sich somit auf den Betrieb und die Bewertung der Ausschuss-Lagers sowie auf die Entscheidung, ein bestimmtes Volumen des Ausschusses entsorgen zu lassen.

\subsubsection{Qualitätssicherung}

Mittels Aufwendungen für Qualitätssicherung kann die Qualität der Prozesse und damit auch die Qualität der Produkte verbessert werden. Dies äußert sich in einer geringeren Rückflussquote (Reklamationen). Diese Zahlungen wirken sich nicht sofort, sondern langfristig aus.

Einwirkende Faktoren auf die Qualität der Prozesse und Qualität der Produkte sind die Aufwendungen für

- $\quad$ ein Qualitäts-Management-System,

- Personalausbildung,

- Forschung und Entwicklung und

- Wartung.

Die Aufwendungen für Qualitäts-Management-System und Personalausbildung ergeben sich aus der Erkenntnis, dass insbesondere der Standardisierung von Prozessen aus qualitätswirtschaftlicher Sicht eine hohe Bedeutung zukommt. Diese Standardisierung wird durch beide Aufwendungen vorangetrieben. ${ }^{16}$ Bezüglich F\&EAufwendungen stellen Heidenberger et al. fest, dass Lernen durch direkte, eigene Erfahrung in der Managementpraxis für die F\&E-Investitionsplanung kaum möglich ist. Die Auswirkungen dieser Investitionen werden schließlich regelmäßig erst Jahre später nachdem der vormalige Entscheidungsträger vielleicht schon das Unternehmen oder zumindest den Verantwortungsbereich gewechselt hat - bzw. an einem anderen Ort sichtbar. ${ }^{162}$ Die Aufwendungen für Wartung haben direkten Einfluss auf die Qualität

\footnotetext{
160 Dies wird erreicht, indem - wie bei den Aufwendungen für Werbung - die über mehrere Perioden geleisteten Zahlungen addiert werden bzw. nur mit einem bestimmten Anteil gewichtet werden.

161 Vgl. hierzu Wilken (Qualität, 1993) Seite 46 f.

162 Vgl. Heidenberger et al. (F\&E-Unternehmensspiel, 2001).
} 
der Produktionsprozesse und damit eine positive Auswirkung auf Durchlaufzeiten bzw. auf die Minimierung des Ausschusses. $\frac{163}{163}$

Die Gesamtqualität bestimmt sich aus der Multiplikation der einzelnen Faktoren, gebildet aufgrund von Investitionen. Dabei ist der Einfluss eines jeden einzelnen Parameters begrenzt. Dies wird in der nachfolgenden Abbildung verdeutlicht. $\frac{164}{1 .}$

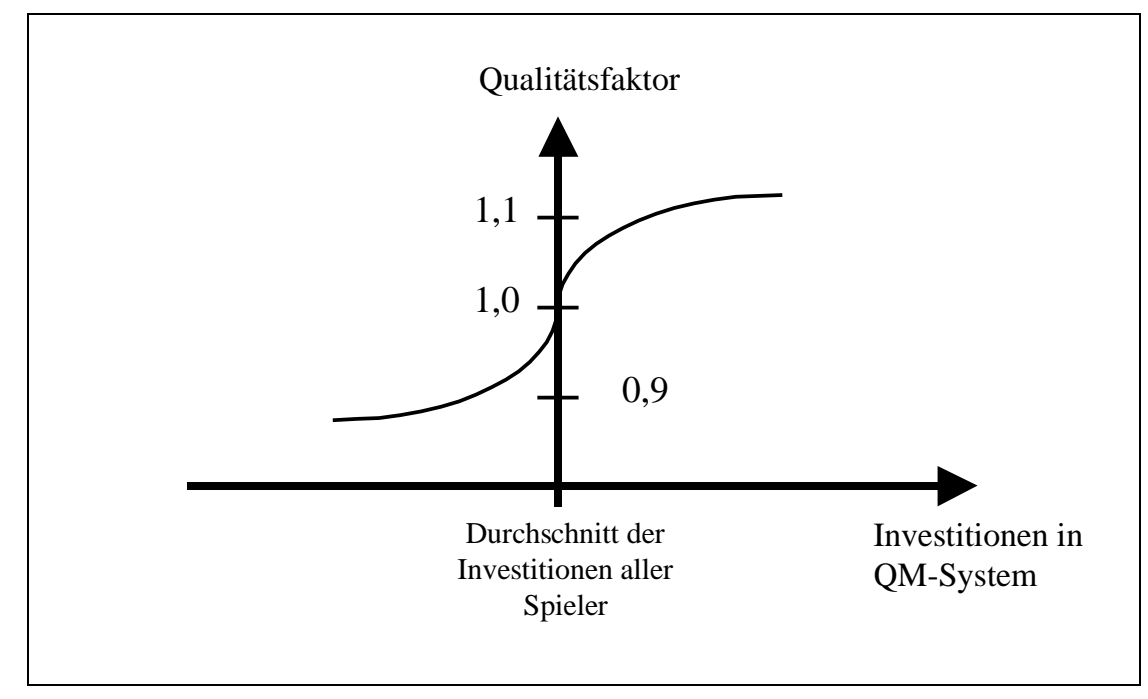

Abb. 23: Wirksamkeit der Qualitätsfaktoren

Die vier unterschiedlichen Aufwendungen für QM-System, Personalfortbildung, F\&E und Wartung haben unterschiedliche Einflüsse. So wirken beispielsweise die Aufwendungen für Wartungen nur auf die Qualität der Produktion (und damit auf den Ausschuss).

\begin{tabular}{|l|c|c|}
\hline Aufwand für & Produktion & Reklamationen \\
\hline QM-System & $\mathrm{X}$ & $\mathrm{X}$ \\
\hline Personalfortbildung & $\mathrm{X}$ & $\mathrm{X}$ \\
\hline F\&E & & $\mathrm{X}$ \\
\hline Wartung & $\mathrm{X}$ & \\
\hline
\end{tabular}

Tab. 23: Einfluss verschiedener Aufwendungen

\footnotetext{
163 Üblicherweise minimiert sich der Verschleißes, was in vorliegendem Planspiel aber nicht berücksichtigt werden soll.

164 Der Kurvenverlauf ist fiktiv festgelegt. Er drückt aus, dass ab einer bestimmten wirksamen Aufwendung für Qualitätsverbesserungen kein weiterer Fortschritt bei der Qualität erzielt werden kann.
} 
Die Berechnung der einzelnen Faktoren erfolgt dabei als Kombination der Aufwendungen vergangener Perioden.

Nachfolgend ein Beispiel für die Bestimmung des Einflussfaktors auf die Produktion:

\begin{tabular}{|l|c|c|c|}
\hline Faktoren & Periode 1 & Periode 2 & $\begin{array}{c}\text { Periode 3 } \\
\text { Planfaktor }\end{array}$ \\
\hline QM-System & 0,958 & 0,967 & 0,963 \\
\hline Personalfortbildung & 1,045 & 1,103 & 1,074 \\
\hline Wartung & 1,327 & 1,275 & 1,301 \\
\hline
\end{tabular}

Tab. 24: Berechnung der Unterstützungsfaktoren in der Planungsphase

Ohne Einfluss der anderen Spieler ergibt sich der (Plan-) Faktor ${ }^{165}$ Produktion zu $0,963 * 1,074 * 1,301=1,346$. Da die Parameter QM-System und Personalfortbildung auch von den Entscheidungen der anderen vier Spieler abhängen, wird der definitive Faktor erst nach Auswertung der Daten am Marktplatz bestimmt. Der Wartungsfaktor ist unabhängig von den Entscheidungen der anderen vier Spieler. Er wird allein durch die Aufwendungen im Verhältnis zur produzierten Menge bestimmt.

In obigem Fall würde sich die Produktion qualitativ verbessern, was sich in einer geringeren Ausschussquote zeigen würde.

Die Reklamationen wirken erst nach einer Periode. Im Planspiel werden die errechneten Reklamationen bereits in die folgende Periode vorgeschrieben.

\subsubsection{Finanzierung}

Es besteht die Möglichkeit, Kredite aufzunehmen und Finanzanlagen zu tätigen. Dabei sind bei erfolgter Kreditaufnahme Zinszahlungen fällig. Diese können durch die Spielleitung in jeder Periode beeinflusst werden. Weiterhin besteht gegebenenfalls die Notwendigkeit, teure Liquiditätskredite in Anspruch $\mathrm{zu}$ nehmen. Diese werden 
automatisch vergeben und in der nächsten Periode zurückbezahlt. Die (Schuld-) Zinsen fallen in der aktuellen Periode an.

Laufen die Geschäfte der Unternehmung gut - ist die Gewinnerzielung nachhaltig und langfristig -, kann das Unternehmen Wertpapiere kaufen. Auch hier kann die Spielleitung die Zinsen für alles Unternehmen gleichermaßen regeln. Ertragszinsen werden erst in der folgenden Periode gutgeschrieben.

Für Kredite und Wertpapiere gilt gleichermaßen, dass der entsprechende Zinssatz in den jeweiligen Grenzen angewandt wird.

\begin{tabular}{|c|c|c|c|c|c|c|}
\hline Bereich & $\begin{array}{c}\text { Regulärer } \\
\text { Kredit p.a. }\end{array}$ & $\begin{array}{c}\text { pro } \\
\text { Periode }\end{array}$ & $\begin{array}{c}\text { Liquiditäts- } \\
\text { Kredit p.a. }\end{array}$ & $\begin{array}{c}\text { pro } \\
\text { Periode }\end{array}$ & $\begin{array}{c}\text { Wertpapier } \\
\text { p.a. }\end{array}$ & $\begin{array}{c}\text { pro } \\
\text { Periode }\end{array}$ \\
\hline$<10$ Mio. $€$ & $9,6 \%$ & $1,60 \%$ & $13,8 \%$ & $2,3 \%$ & $6,0 \%$ & $1,0 \%$ \\
\hline$<20$ Mio. $€$ & $10,5 \%$ & $1,75 \%$ & $15,0 \%$ & $2,5 \%$ & $7,2 \%$ & $1,2 \%$ \\
\hline$>20$ Mio. $€$ & $11,4 \%$ & $1,90 \%$ & $17,4 \%$ & $2,9 \%$ & $8,4 \%$ & $1,4 \%$ \\
\hline
\end{tabular}

Tab. 25: Zinssätze für Finanzierungen Kreditzinsen

Die Zinsen berechnen sich wie folgt: Beträgt das reguläre Kreditvolumen in der aktuellen Periode z.B. 25 Mio. $€$, so fallen für 10 Mio. $€$ Zinsen in Höhe von $160.000 €$, für weitere 10 Mio. $€$ Zinsen in Höhe von $175.000 €$ und für die restlichen 5 Mio. $€$ Zinsen in Höhe von $95.000 €$ an. Ins Summe werden in dieser Periode $430.000 €$ an Zinsen fällig.

\subsubsection{Personalwirtschaft}

\subsubsection{Fach- und Hilfsarbeiter}

Prinzipiell benötigt man Fach- und Hilfsarbeiter: Facharbeiter in der Produktion, Hilfsarbeiter in der Montage und für die innerbetriebliche Logistik.

165 Für die Planungsphase kann der Einfluss der vier Konkurrenten nicht ermittelt werden. Deswegen wird der Planfaktor der aktuellen Periode (hier Periode 3) als Mittelwert der beiden vorhergehenden Perioden 
Die Einstellung (Entlassung) bringt jeweils Kosten bei Facharbeitern in Höhe von 1.500,- $€(2.000,-€)$ und bei Hilfsarbeitern in Höhe von 700,- $€(1.000,-€)$ mit sich. Die Arbeiter können in der nächsten Periode eingesetzt bzw. entlassen werden. Eine Beschränkung für Einstellungen und Entlassungen ist vorgegeben: Der Personalstand ändert sich in jeder Gruppe (Fach- bzw. Hilfsarbeiter) um maximal 10 Prozent. Löhne und Gehälter betragen anfangs 3.000,- € für Facharbeiter und 2.000,- $€$ für Hilfsarbeiter.

\begin{tabular}{|l|c|c|}
\cline { 2 - 3 } \multicolumn{1}{c|}{} & Facharbeiter & Hilfsarbeiter \\
\hline Lohn je Periode & $3.000 €$ & $2.000 €$ \\
\hline Einstellung & $1.500 €$ & $700 €$ \\
\hline Entlassung & $2.000 €$ & $1.000 €$ \\
\hline
\end{tabular}

Tab. 26: Lohn-, Einstellungs- und Entlassungskosten

Die Mitarbeiter besitzen bestimmte Kapazitäten. ${ }^{166}$ Facharbeiter sind leistungsfähiger als Hilfsarbeiter, was sich in der um 50 Prozent höheren Kapazität ausdrückt.

\begin{tabular}{|l|c|c|}
\cline { 2 - 3 } \multicolumn{1}{c|}{} & Facharbeiter & Hilfsarbeiter \\
\hline Kapazität pro Stunde & 12 & 8 \\
\hline Kapazität pro Tag & 96 & 64 \\
\hline Kapazität pro Periode & 3.840 & 2.560 \\
\hline
\end{tabular}

Tab. 27: Kapazitäten pro Fach- und Hilfsarbeiter

Für bestimmte Vorgänge werden bestimmte Personalkapazitäten benötigt.

\begin{tabular}{|l|l|c|c|}
\cline { 2 - 4 } \multicolumn{1}{c|}{} & Vorgang & benötige Kapazität & Einheit \\
\hline Hilfsarbeiter & 0,001 & $\mathrm{Cap} / \mathrm{m}$ \\
& Produktion Produkt 1 & 0,04 & $\mathrm{Cap} / \mathrm{St}$ \\
& Produktion Produkt & 0,02 & $\mathrm{Cap} / \mathrm{m}$ \\
& Montage Singulus & 0,05 & $\mathrm{Cap} / \mathrm{St}$ \\
\hline & Transport Produkt 1 \& 2 & 0,06 & $\mathrm{Cap} / \mathrm{m}^{3}$ \\
& Abladen Produkt 3 \& & 0,08 & $\mathrm{Cap} / \mathrm{m}^{3}$ \\
& Transport Halbfabrikate & 0,06 & $\mathrm{Cap} / \mathrm{m}^{3}$ \\
& Transport Artikel & 0,07 & $\mathrm{Cap} / \mathrm{m}^{3}$ \\
& Beladen LKW & 0,07 & $\mathrm{Cap} / \mathrm{m}^{3}$ \\
& Transport Recyclat & 1,00 & $\mathrm{Cap} / \mathrm{m}^{3}$ \\
& Abfrachtung Ausschuss & 0,05 & $\mathrm{Cap} / \mathrm{m}^{3}$ \\
\hline
\end{tabular}

Tab. 28: Benötigte Personalkapazitäten 
Verknüpft man die beiden Tabellen miteinander so zeigt sich, dass ein Facharbeiter in einer Stunde $12.000 \mathrm{~m}$ des Produktes 1 herstellen kann. Dies errechnet sich aus $12 \frac{\mathrm{Cap}}{\mathrm{h}} * \frac{1}{0,001 \frac{\mathrm{Cap}}{\mathrm{m}}}=12.000 \frac{\mathrm{m}}{\mathrm{h}}$.

Die aufgrund des Personalumfangs zur Verfügung stehenden Kapazitäten verteilen sich auf die nötigen Vorgänge. Sollten zuwenig Kapazitäten vorhanden sein, werden Leihkapazitäten bzw. Überstunden in Anspruch genommen. Die dafür entstehenden Kosten liegen 30 Prozent über den variablen (Lohn-) Kosten der fest eingestellten Mitarbeiter.

Die Personalkosten für die Produktion werden in der GuV unter Umsatzkosten gebucht.

\subsubsection{Sozialleistungen}

Wie bei jedem Unternehmen ist in jeder Periode eine bestimmte Anzahl von Mitarbeitern krank oder arbeitsunfähig. Es gilt der Einfachheit halber die Annahme, dass der Krankenstand progressiv zum Kostensatz für Sozialleistungen ist.

Sozialleistungen setzen sich aus drei unterschiedlichen Einzelleistungen zusammen:

1. Gesetzliche Sozialleistungen, wie z.B. Arbeitgeberanteile an den Sozialversicherungen,

2. vertragliche Sozialleistungen, wie z.B. Urlaubs- oder Weihnachtsgeld und

3. freiwillige Sozialleistungen, wie z.B. Sonderzahlungen oder Geschenke an die Mitarbeiter.

Es wird davon ausgegangen, dass sich der Krankenstand mit einem höheren Kostensatz für Sozialleistungen reduziert und damit der Beschäftigungsgrad steigt. ${ }^{167}$ Dadurch stehen mehr produktive Mitarbeiter zur Verfügung.

167 Anmerkung: Der Grad der Beschäftigung lässt sich aufgrund natürlicher Gegebenheiten auf maximal 97 Prozent steigern. 
Der Beschäftigungsgrad beträgt anfangs 90 Prozent und kann irgendwo zwischen 85 Prozent und 97 Prozent liegen. Dies ist von den Aufwendungen für Sozialleistungen und für Aufwendungen für Personalweiterentwicklung abhängig. Der Kostensatz für Sozialleistungen beträgt anfangs 43 Prozent. Er ist variabel und wirkt sich direkt auf den Krankenstand aus, wobei der Einfluss begrenzt ist.

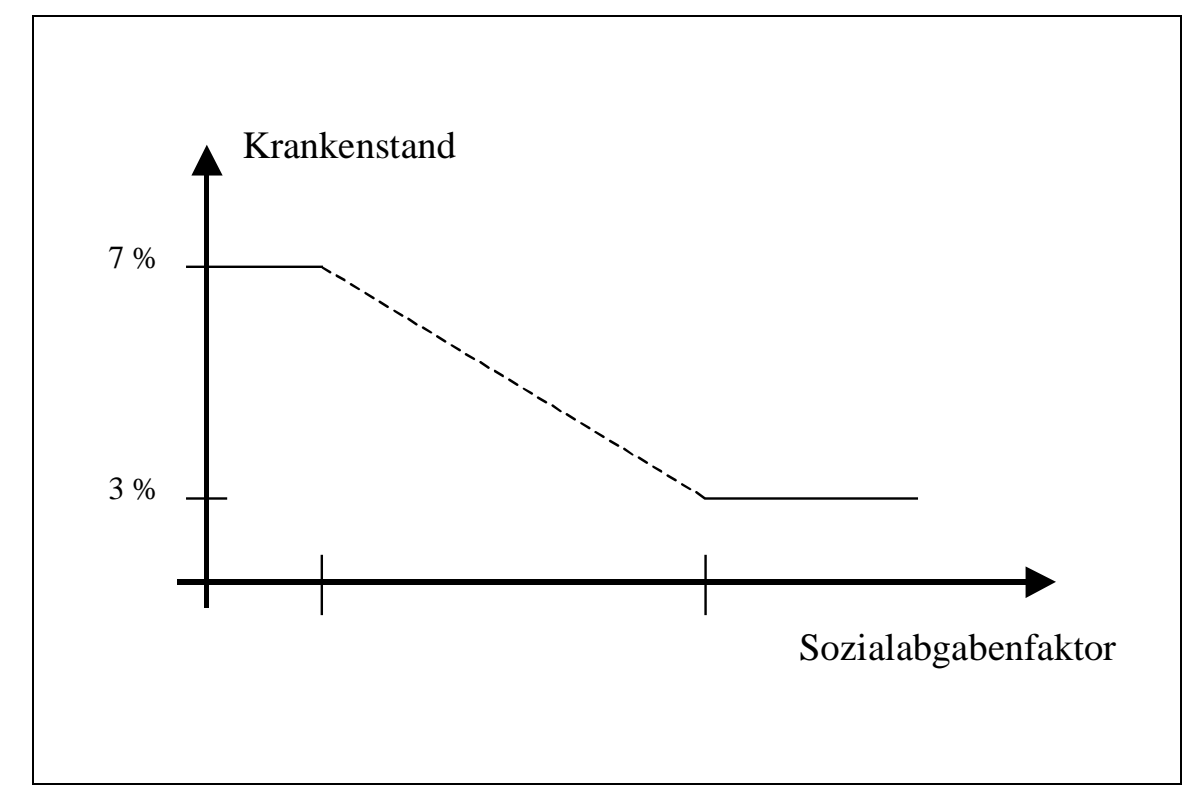

Abb. 24: Einfluss der Sozialabgaben auf den Krankenstand

Dabei ist der Krankenstand nicht linear, sondern mit einer zufälligen Komponente überlagert. Dies wird in der Graphik durch die gestrichelte Linie dargestellt.

\subsubsection{Verwaltung und Vorstand}

Wie bei jedem Unternehmen fallen bei LogisticPLUS Kosten der Verwaltung an. Diese sind abhängig erstens vom erzielten Umsatz und zweitens von der Anzahl der beschäftigten Mitarbeiter.

Die umsatzabhängigen Kosten der Verwaltung beziehen sich auf dem Bruttoumsatz. Ursache dieser Kosten sind Aufwendungen im administrativen Bereich: z.B. Beschaffung, Lagerkontrolle oder Rechnungswesen (Buchhaltung). 


\begin{tabular}{|c|c|}
\hline Umsatz [Mio. $€$ ] & $\begin{array}{c}\text { Kosten der Verwaltung [€] } \\
\text { je 1 Mio. } € \text { Umsatz }\end{array}$ \\
\hline $0<\mathrm{x}<30$ & 13.000 \\
\hline $30<\mathrm{x}<40$ & 10.000 \\
\hline $40<\mathrm{x}$ & 8.000 \\
\hline
\end{tabular}

Tab. 29: Umsatzabhängige Kosten der Verwaltung

Beispiel: Bei einem Umsatz von 35,5 Mio. € fallen Kosten der Verwaltung in Höhe von 443.000,- $€$ an. Diese setzen sich zusammen aus Kosten für die ersten 30 Mio. in Höhe von $30 * 13.000,-=390.000,-€$. Die weiteren 5,3 Mio. $€$ verursachen zusätzliche Kosten der Verwaltung in Höhe von 5,3 $* 10.000,-€=53.000,-€$.

Die personalabhängigen Kosten der Verwaltung entstehen durch umfangreiche Personalarbeit. So werden z.B. ab bestimmten Größenordnungen zusätzliche Personalsachbearbeiter und Büros benötigt. Aus diesem Grunde berechnen sich die personalabhängigen Kosten aus einem fixen und einem variablen Anteil. Der variable Anteil gilt jedoch nur für die Anzahl der Mitarbeiter, der über der jeweiligen „unteren“ Grenze liegt.

\begin{tabular}{|c|c|c|}
\hline $\begin{array}{c}\text { Anzahl } \\
\text { der Mitarbeiter }\end{array}$ & Fixkosten & $\begin{array}{c}\text { Kosten je } \\
\text { Mitarbeiter }\end{array}$ \\
\hline $0<\mathrm{x}<700$ & $450.000 €$ & \\
\hline $700<\mathrm{x}<1.100$ & $1.000 .000 €$ & $500 €$ \\
\hline $1.100<\mathrm{x}$ & $1.200 .000 €$ & $300 €$ \\
\hline
\end{tabular}

Tab. 30: Personalabhängige Kosten der Verwaltung

Beispiel: Bei einer Mitarbeiterzahl von 961 Beschäftigten fallen für 700 Mitarbeiter Fixkosten in Höhe von 1 Mio. $€$ und für 261 Mitarbeiter variable Kosten in Höhe von 130.500,- $€$ an. Die Gesamtsumme der personalabhängigen Kosten der Verwaltung beträgt somit $1.130 .500 €$.

Die Kosten der Verwaltung werden in der GuV unter „Vertriebskosten“, in der Liquiditätsrechnung als eigener Auszahlungspunkt „Kosten der Verwaltung“ angegeben. 
Das Unternehmen wird von einem (mehrköpfiger Team-) Vorstand geführt. Die Summe der Vorstandsgehälter beträgt anfangs 36.000,- $€$ pro Periode. Es wird in der Gewinn- und Verlustrechnung unter „Allgemeine Verwaltungskosten“ gebucht.

\subsubsection{Eingabemasken für verschiedene Bereiche}

Der Aufbau des Computerprogramms ist selbsterklärend. Nachfolgend werden die Bildschirmmasken des Entscheidungsbereiches dargestellt. Die restlichen Masken folgen bei den entsprechenden Kapiteln.

Im Eingabebereich Absatz / Marketing müssen für Singulus und Combilus die jeweiligen Preise und Planmengen auf allen drei Märkten angegeben werden. Welche Mengen tatsächlich realisiert werden, ergibt sich erst nach der Simulation. Weiterhin müssen die Ausgaben für Werbung und Marktforschung festgelegt werden.

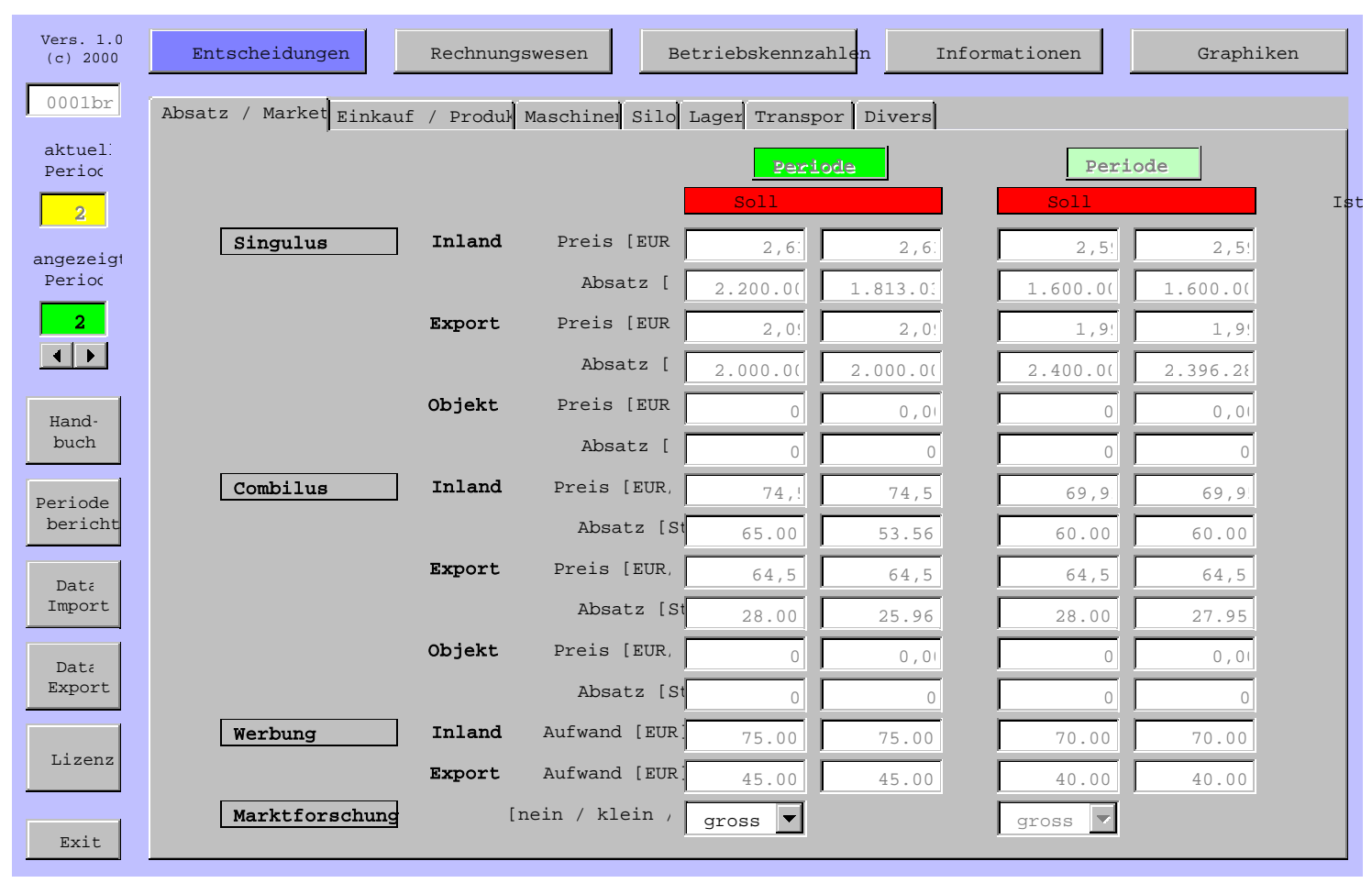

Abb. 25: Eingabemaske Absatz und Marketing

Die Felder der Maske werden durch die Planspielteilnehmer ausgefüllt. Die Sollwerte sind die jeweiligen Planwerte der Spieler, bevor eine Simulation durchgeführt wurde. 
Die Ist-Werte sind je nach Periodenstand die Werte, die entweder durch die Simulation wegen fehlender Kapazitäten nach unten beschränkt wurden oder die nach dem Durchlauf bei der Spielleitung in Abhängigkeit der Marktsituation tatsächlich angefallenen sind. In diesem Beispiel - wir befinden uns in Periode 2, was im linken oberen Bereich angezeigt wird - zeigt sich, dass z.B. der geplante Absatz von 2,2 Mio. m Singulus im Inland nicht ausgeführt werden kann. Es können vermutlich nur 1,813 Mio. m abgesetzt werden. Die Ursachen hierfür können verschieden sein: zu geringer Lagerbestand, zu wenig Personal, Gabelstapler oder LKW stehen zur Verfügung. Der aktuelle Markt kann in dieser Situation noch keinen Einfluss ausüben, da die Simulation durch die Spielleitung noch nicht erfolgt ist. Dies ist allerdings in der vergangenen Periode 1 der Fall gewesen: Von den geplanten 2,4 Mio. m im Export konnten 2,396 Mio. m verkauft werden. 
Im Bereich Einkauf / Produktion werden die geplanten Mengen der zwei Rohstoffe und der zwei Zukaufprodukte festgelegt. Weiterhin sind die geplanten Produktionsmengen der Produkte 1 und 2 festzulegen sowie die Planmengen der Montageaktivitäten der VK-Artikel Singulus und Combilus. Recycling- bzw. Entsorgungsmengen runden diese Bereich ab.

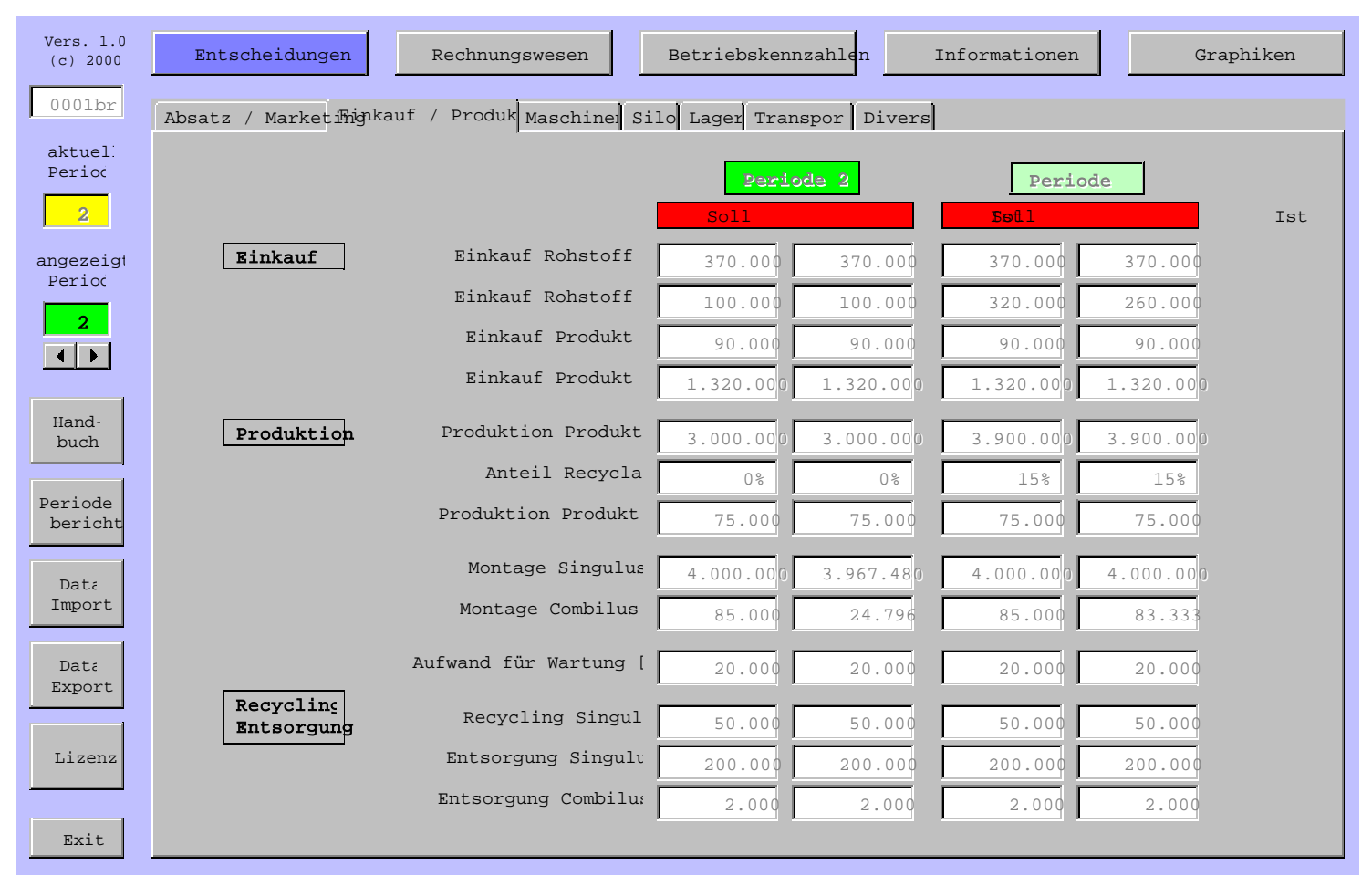

Abb. 26: Eingabemaske Einkauf und Produktion

Ebenso wie in der vorhergehenden Maske Die Einkaufs- und Produktionsmengen müssen durch die Spieler in getrennter Rechnung ermittelt werden.

Rohstoffe und Halbfabrikate (Produkt 3 und 4) treffen in der Periode nach der Bestellung ein und werden dann auch bezahlt. Sie können nicht weiterverkauft werden. 
In der Produktion stehen als Maschinen für die zwei Produktionsbereiche 1 und 2 sowie für die Montage insgesamt drei unterschiedliche Typen zur Verfügung. Für jeden der drei Typen stehen insgesamt acht Plätze als Installationsplätze zur Verfügung.

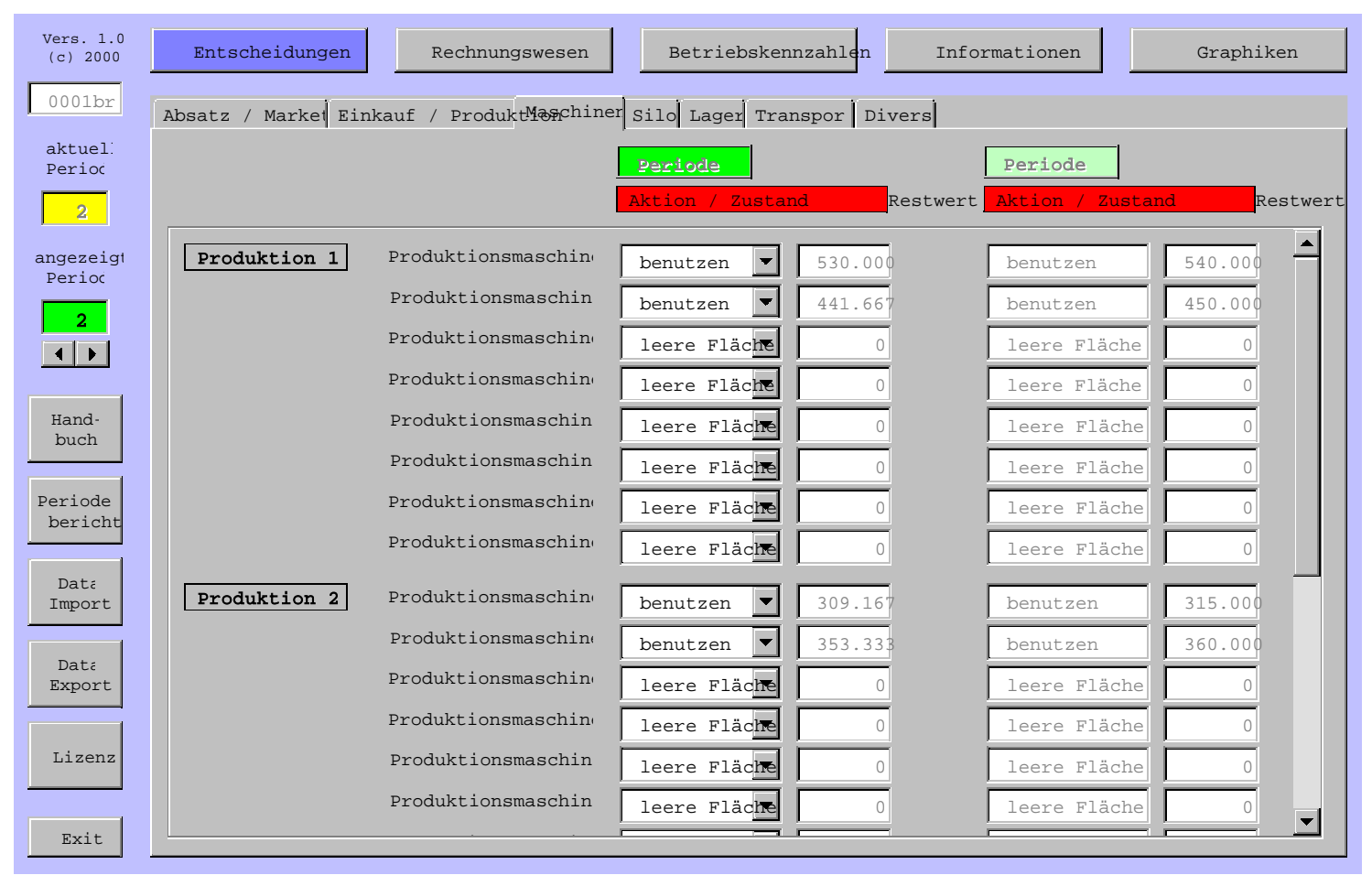

Abb. 27: Eingabemaske Maschinen

Die Maske zeigt beispielweise eine Situation mit zwei eingesetzten Maschinen im Bereich der Produktion 1 und ebenso vielen im Bereich der Produktion 2. Dargestellt wird neben dem Zustand der Fläche (leere Fläche, beschaffen, benutzen oder verkaufen) der aktuelle Restwert einer vorhandenen Maschine. Wenn die Aufstellung weiterer Maschinen vorgenommen werden soll, ist dies in das Feld einer freien Fläche einzutragen. Die Beschaffung einer neuen und der Verkauf einer Maschine wird in der folgenden Periode wirksam, die Bezahlung erfolgt ebenfalls in der folgenden Periode. Maschinen werden über 60 Perioden abgeschrieben, eine Weiterverwendung ist nicht möglich, die nötige Entsorgung kostenfrei.

168 Bei obiger Maske sind die acht Plätze der Montagemaschinen nicht direkt erkennbar. Mit der Bildlaufleiste auf der rechten Seite kann jedoch zu diesem Bereich gescrollt werden. 
Bei den Silos ergibt sich ein ähnliches Bild wie bei den Maschinen. Hier stehen für die beiden Rohstoffe jeweils sechs, für das Recyclat insgesamt zwei Silofundamente zur Verfügung. Die möglichen Entscheidungen lauten hier leere Fläche, kaufen, benutzen und vermieten. ${ }^{169}$ Auch hier werden die Restwerte automatisch berechnet.

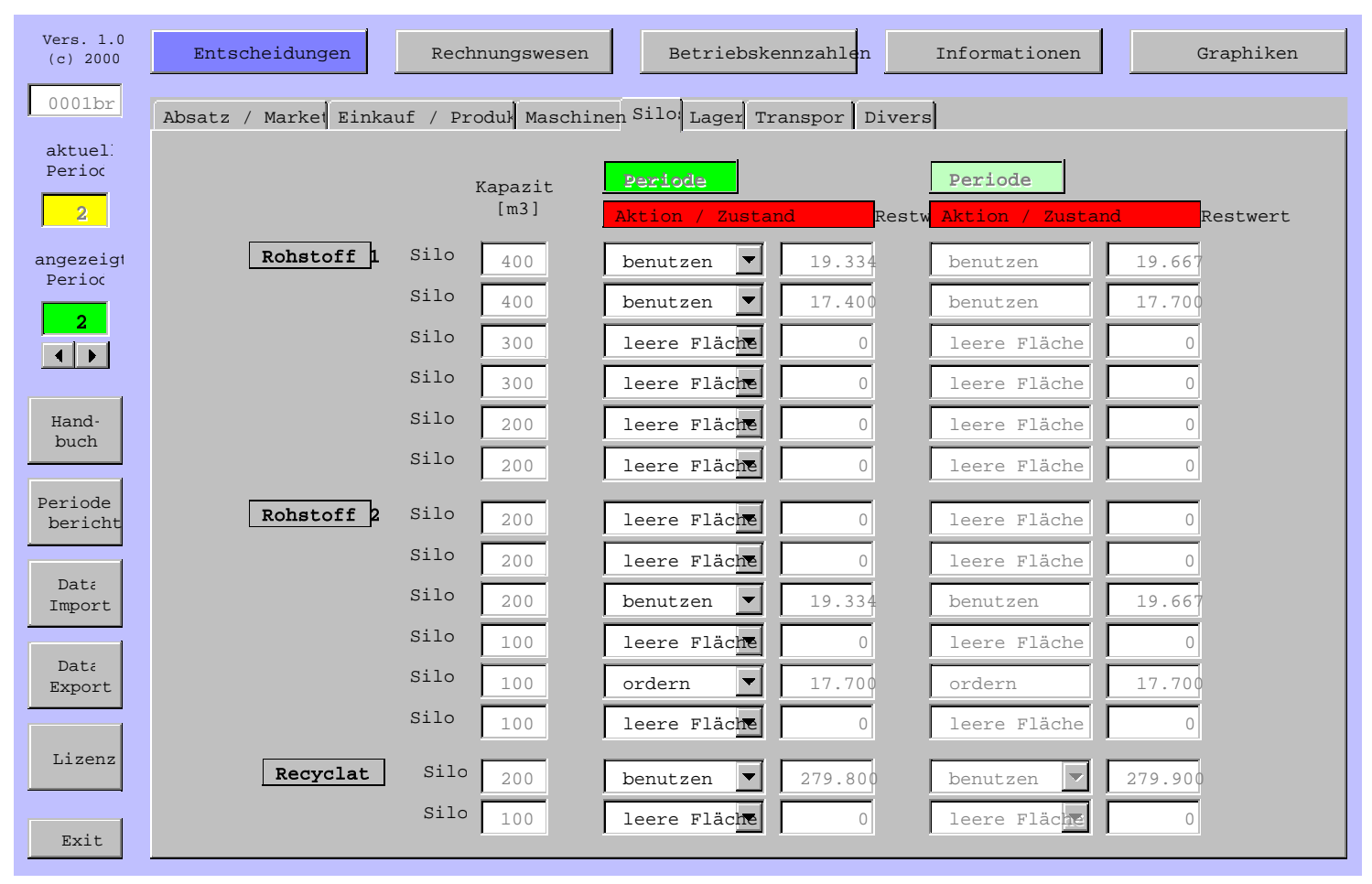

Abb. 28: Eingabemaske Silos

Die Bestellung der Rohstoffe dient der Sicherung der zukünftigen Produktion. Gegebenenfalls können auch erwartete Preissteigerungen umgangen werden. Somit ist eine optimale Anzahl von Silos nicht errechenbar, sondern vielmehr von der Einschätzung der weiteren Entwicklung der Marktsituation durch die Spielergruppe abhängig.

169 Silos können nicht verkauft werden. Allerdings besteht wie bei Lagerhallen die Möglichkeit, diese an externe Firmen zu Festpreisen zu vermieten. Informationen über die Preise finden sich im Bereich Informationen. 
Im Bereich Lager sind für Halbfabrikate und VK-Artikel ähnlich wie bei den Maschinen und Silos jeweils vier Flächen vorgesehen. Dies sind Fundamente, auf die Lager aufgebaut werden können. Die möglichen Aktionen bzw. Zustände lauten hier wie bei den Silos leere Fläche, kaufen, benutzen und vermieten. Der Restwert der Lager wird automatisch berechnet, die Nutzungsdauer beträgt 50 Jahre. Abgeschriebene Lager werden kostenfrei entsorgt.

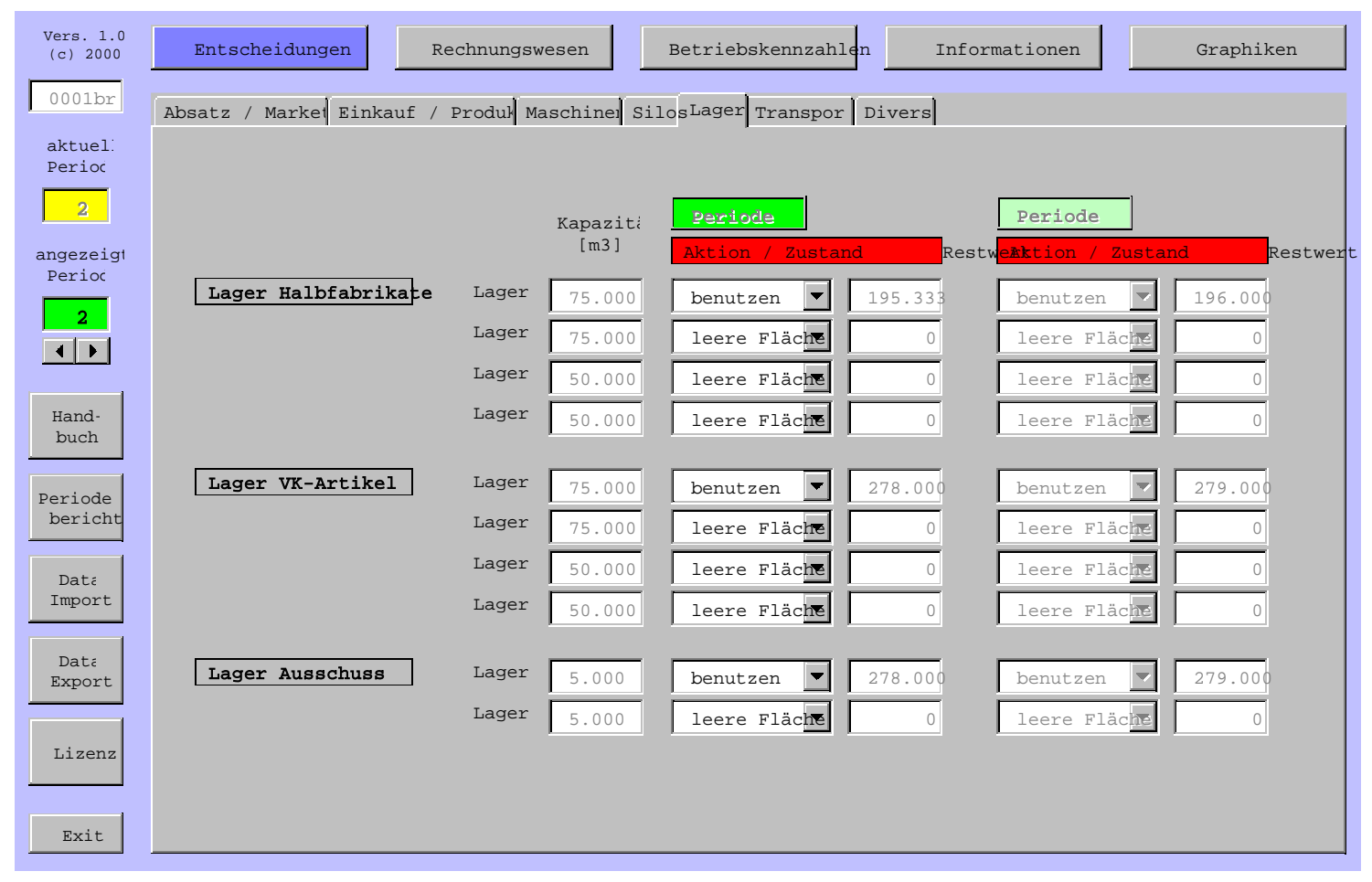

Abb. 29: Eingabemaske Lager

Die Lager werden in der aktuellen Periode $(\mathrm{T}=0)$ bestellt. Sie werden in der folgenden Periode $(\mathrm{T}=1)$ bezahlt und gleichzeitig aufgebaut und stehen also erst in der übernächsten $(\mathrm{T}=2)$ Periode zur Verfügung. Lager können wie Silos auch nicht verkauft sondern vermietet werden. Die vermieteten Lagervolumen stehen ab der nächsten Periode nicht mehr zur Verfügung, die Mieteinnahmen erfolgen ab diesem Zeitpunkt. 
Im Bereich Transporte muss jeder Spieler Lastkraftwagen bestellen, um seine (geplanten) VK-Artikel auf den drei Märkten Inland, Export und ggf. Ausschreibung abzusetzen. Dabei ist zu beachten, dass die Planabsatzmengen in der Regel nicht exakt eintreffen. Sollte z.B. einem Spieler aufgrund seines unterdurchschnittlichen Preises ein höheres Volumen zugewiesen werden so kann die Bestellmenge der Transportmittel die mögliche Absatzmenge einschränken. Dieselben Überlegungen gelten für die Gabelstapler, die für die innerbetrieblichen Transporte benötigt werden. Hier besteht die Möglichkeit Gabelstapler zu kaufen, zu verkaufen oder zeitweilig anzumieten.

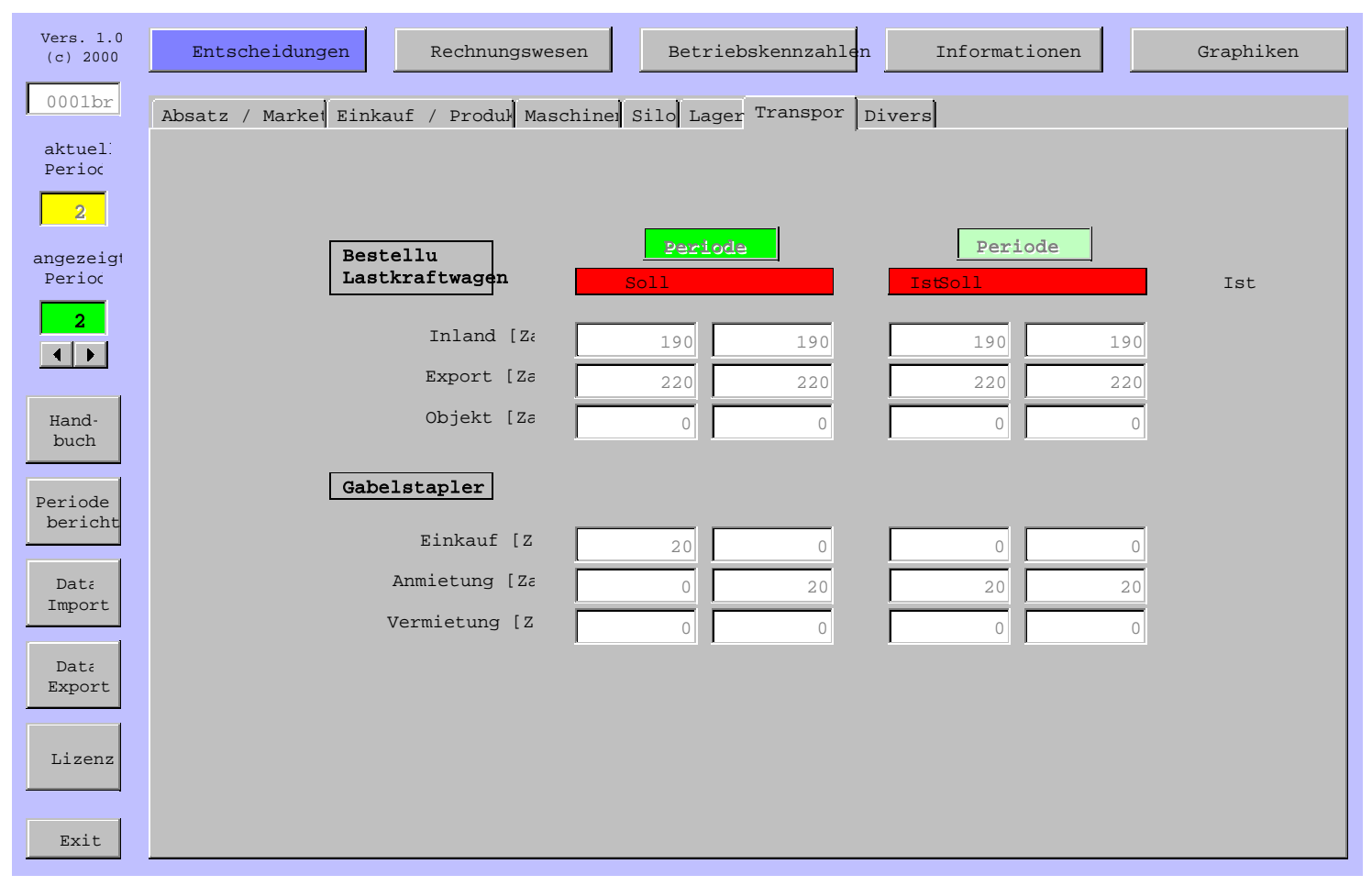

Abb. 30: Eingabemaske Transporte

Hier werden unter Ist- immer die geplanten Soll-Werte angezeigt. Eine Auswertung über die Auslastung der LKW erhält man in der Maske Transporte im Menü Betriebskennzahlen. 
Im letzten Eingabebereich Diverse werden die noch ausstehenden Entscheidungen zusammengefasst. Dies sind Entscheidungen über Investitionen in die Qualität der Produkte bzw. Prozesse, Entscheidungen über Finanzen und Entscheidungen über den Personalbestand bzw. den Sozialkostenfaktor.

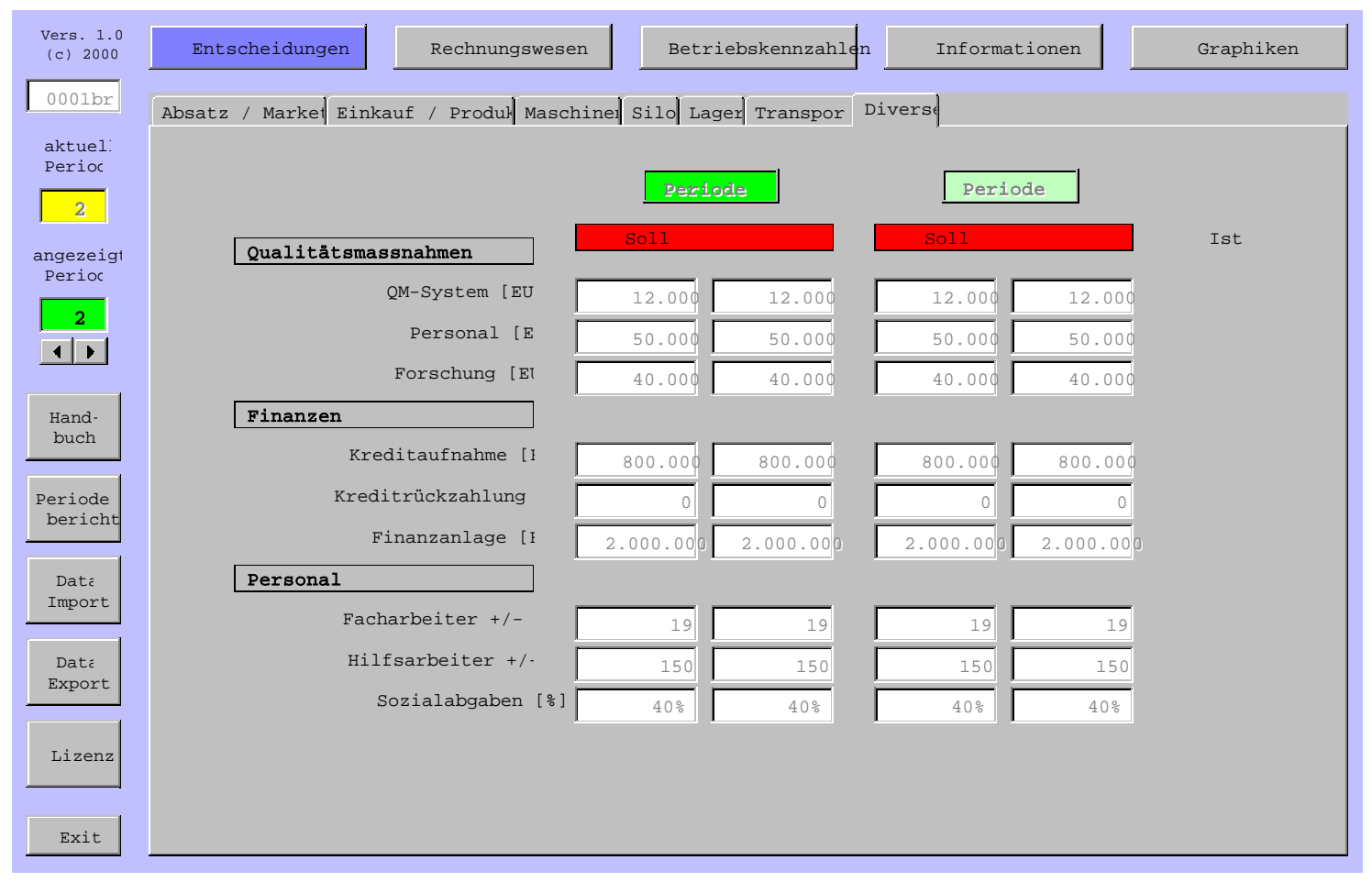

Abb. 31: Eingabenmaske Diverse

Sollte der aktuelle Kassenbestand inklusive geplanter Kredite für die geplanten Ausgaben nicht ausreichen, wird automatisch ein Liquiditätskredit in Anspruch genommen. Dies würde man in vorliegender Maske in einer der Ist-Spalten sehen. Die absolute Kredithöhe ist im Planspiel beschränkt und hängt u.a. von der Eigenkapitalhöhe ab.

Die Personalzahlen sind immer relative Veränderungen, es gibt bei Entlassungen und Einstellungen Ober- und Untergrenzen. Mit der Einstellung und Entlassung entstehen Kosten für das Unternehmen, die jeweils sofort wirksam werden. 


\subsection{Interne und externe Rechnungsinformationen}

\subsubsection{Kostenrechnung}

In der Betriebswirtschaftslehre unterscheidet man Herstellkosten und Herstellungskosten. ${ }^{170}$ Herstellkosten sind ein Begriff der Kostenrechnung. Hierunter werden alle zur Herstellung eines Produktes anfallenden Kosten verstanden. Sie setzen sich zusammen aus Materialkosten (Einzelkosten + Gemeinkosten), Fertigungskosten (Einzelkosten + Gemeinkosten) sowie aus den Sondereinzelkosten der Fertigung. Herstellkosten können sowohl auf Basis der vollen Kosten als auch lediglich auf Basis variabler Kosten berechnet werden. Herstellungskosten sind ein Begriff der Finanzbuchhaltung. Die gesetzliche Definition findet sich in $\S 255$ Absatz 2 Handelsgesetzbuch. Dieser liegen als Rechnungsgröße nicht Kosten, wie der Name „Herstellungskosten“ vermuten lässt, sondern Aufwendungen zugrunde.

Die Kostenrechnung beim Planspiel hat zwei wesentliche Aufgaben:

1. Berechnung der Herstellungs- und im weiteren der Selbstkosten und damit der mindestens zu erzielenden Verkaufspreise. Die Kostenrechnung ist das zentrale Mittel, um die erbrachten Leistungen (hier die Produkterstellung) finanziell $\mathrm{zu}$ bewerten. Sie wird so aufgestellt, dass der Spieler einen möglichst umfassenden Einblick in die Einzel- und Gemeinkosten sowie in die Kostentreiber der beiden zu fertigen Halb- und Fertigfabrikate erhält.

2. Bewertung der Bestände an Halbfabrikaten und VK-Artikel. Diese Bestände werden für die Bilanz benötigt. Diese ist ein ebenfalls sehr wichtiges und hilfreiches Element, um insbesondere den Überblick über die Situation des Umlaufvermögens und damit der Bestandführung zu bekommen.

\footnotetext{
170 Hoitsch (Kostenrechnung, 1996) Seite 387.
} 
In der Realität der deutschen Gesetzgebung wird hierbei bei der Handels- bzw. Steuerbilanz eine wesentliche Unterscheidung bezüglich der Aktivierungsfähigkeit bzw. die Aktivierungspflicht von bestimmten Kosten getroffen.

\begin{tabular}{|c|c|c|c|}
\hline & Kostenkomponenten & Handelsbilanz & Steuerbilanz \\
\hline $\begin{array}{l}+ \\
+\end{array}$ & $\begin{array}{l}\text { Fertigungsmaterial } \\
\text { Fertigungslöhne } \\
\text { Sondereinzelkosten Fertigung }\end{array}$ & muss & muss \\
\hline$=$ & Herstellungskosten I & $\begin{array}{l}\text { Ansatz möglich } \\
\text { (Untergrenze) }\end{array}$ & $\begin{array}{l}\text { Ansatz nicht } \\
\text { möglich }\end{array}$ \\
\hline $\begin{array}{l}+ \\
+\end{array}$ & $\begin{array}{l}\text { variable Materialgemeinkosten } \\
\text { variable Fertigungsgemeinkosten * }\end{array}$ & kann & muss \\
\hline$=$ & Herstellungskosten II & Ansatz möglich & $\begin{array}{l}\text { Ansatz nicht } \\
\text { möglich }\end{array}$ \\
\hline $\begin{array}{l}+ \\
+ \\
+\end{array}$ & $\begin{array}{l}\text { Sondergemeinkosten Fertigung } \\
\text { fixe Materialgemeinkosten } \\
\text { fixe Fertigungsgemeinkosten } * *\end{array}$ & kann & muss \\
\hline$=$ & Herstellungskosten III & Ansatz möglich & $\begin{array}{l}\text { Ansatz möglich } \\
\text { (Untergrenze) }\end{array}$ \\
\hline $\begin{array}{l}+ \\
+\end{array}$ & $\begin{array}{l}\text { fixe Fertigungsgemeinkosten } \\
\text { Verwaltungsgemeinkosten }\end{array}$ & kann & kann \\
\hline$=$ & Herstellungskosten IV & $\begin{array}{l}\text { Ansatz möglich } \\
\text { (Obergrenze) }\end{array}$ & $\begin{array}{c}\text { Ansatz möglich } \\
\text { (Obergrenze) }\end{array}$ \\
\hline
\end{tabular}

* Werteverbrauch des fertigungsbedingten Anlagevermögens ** $\quad$ Z.B. Zinsen für fertigungsbedingtes Fremdkapital, Aufwendungen für freiwillige soziale Leistungen und für betriebliche Altersversorgung

Tab. 31: Ermittlung der Herstellungskosten in Handels- und Steuerbilan 27

Anmerkung: Die im Planspiel benutzten Kostenbegriffe sind immer Herstellungskosten, da kalkulatorische Kosten nicht berücksichtigt werden und damit Kosten gleich den betrieblichen Aufwendungen sind. Im nachfolgenden werden diese Begriffe detaillierter beschrieben.

\subsubsection{Bewertungsspielräume}

Als Erfolgsrechnung wird das Umsatzkostenverfahren ${ }^{172}$ gewählt. Hierbei werden den Erlösen der abgesetzten Erzeugnisse die Selbstkosten der abgesetzten Erzeugnisse

171 Bea et al. (ABWL, 2000), Band 2, Seite 349.

172 Zur Definition vgl. Bea et al. (ABWL, 2000) Band 2, Seite 546, Eversheim / Schuh (Betriebshütte, 1996) Seite 823, Schierenbeck (BWL, 2000) Seite 500 oder Wöhe (ABWL, 2000) Seite 1140. Das alternative Kostenverfahren ist das sogenannte Gesamtkostenverfahren. 
gegenüber gestellt. So kann auf einen Ansatz von Bestandsveränderungen verzichtet werden.

Innerhalb der Planungsphase - d.h. der Spieler hat seine Daten noch nicht an die Spielleitung geschickt - werden sämtliche Kenngrößen aufgrund der Planwerte des Spielers berechnet. Als Kosten fallen dann z.B. die Plankosten an. Nachdem die Daten von der Spielleitung zurückgekommen sind und somit eine neue Periode begonnen hat, werden die Plandaten zu Ist-Daten. So werden insbesondere im Absatzbereich vermutlich größere Änderungen gegenüber der Planungsphase auftreten.

Abschreibungen erfolgen linear. Kosten werden möglichst direkt zugeschlagen. Dabei wird immer der entsprechende Einzelkostenwert als Basis herangezogen. Beispiel: Die Abschreibungen der Silos können den beiden selbst hergestellten Produkten 1 und 2 eindeutig zugeschlagen werden, da in einem Silo jeweils nur ein Rohstoff bzw. Recyclat gelagert wird.

Es werden keine kalkulatorischen Eigenkapitalzinsen ausgewiesen, ebenso werden keine Wagniskosten z.B. für Betriebsunterbrechungen angesetzt. Aufwendungen für Fremdkapital werden als Sondergemeinkosten bewertet und fließen damit in die Kalkulation der Verkaufsartikel ein.

Die anfallenden Gemeinkosten werden jeweils nach den dazugehörigen Einzelkosten bzw. nach den Herstell- oder Herstellungskosten zugeschlagen.

Aufgrund der zweistufigen Produktion sowie der Notwendigkeit der Bewertung von Halbfabrikaten und Fertigfabrikaten in der Bilanz sind vier Kalkulationen aufzusetzen:

1. Herstellungskosten A für die beiden in eigener Regie hergestellten Produkte 1 und 2,

2. Herstellungskosten B für Berechnung der Herstellkosten der Montage von Singulus und Combilus,

3. Herstellungskosten $\mathrm{C}$ als Herstellungskosten $\mathrm{B}$ plus anteilige Gemeinkosten Verwaltung, Gemeinkosten Weiterentwicklung, Sondergemeinkosten Reklamationen sowie weitere für die Bilanz aktvierfähigen Kosten und 
4. Selbstkosten als Maßgabe für den Verkaufspreis. Hierin sind insbesondere die Vertriebskosten enthalten.

\subsubsection{Kalkulation der Halbfabrikate (Herstellungskosten A)}

Die Herstellungskosten der Halbfabrikate bestehen aus den zurechnungsfähigen Einzelkosten sowie den nur für diesen Bereich des Unternehmens anfallenden Gemeinkosten. Hierunter fallen z.B. Abschreibungen für das Halbfabrikatlager oder Kosten der innerbetrieblichen Transporte in diesem Bereich.

\begin{tabular}{|l|c|l|}
\hline Kostenart & & Daten \\
\hline Einzelkosten Material & 1 & Kosten für Rohstoff und Recyclat \\
\hline Gemeinkosten Material & 2 & Abschreibungen auf Rohstoff- und Recyclat-Silos \\
\hline Einzelkosten Fertigung & 3 & $\begin{array}{l}\text { Maschinenkosten Produktion, } \\
\text { Personalkosten Facharbeiter }\end{array}$ \\
\hline Gemeinkosten Fertigung & 4 & $\begin{array}{l}\text { Abschreibungen auf Maschinen und Läger, } \\
\text { innerbetriebliche Transportkosten (Gabelstapler), } \\
\text { Personalkosten Hilfsarbeiter }\end{array}$ \\
\hline Herstellungskosten A & & \\
\hline
\end{tabular}

Tab. 32: Kalkulation der Halbfabrikate (Herstellungskosten A)

\section{Anmerkungen:}

1. Recyclat wird mit den beim Recyclingprozess anfallenden Kosten pro kg bewertet. Diese werden im Planspiel und dem Punkt „Marktpreise“ periodisch durch die Spielleitung festgelegt.

2. Die Abschreibungen auf Rohstoff- und Recyclat-Silos können den beiden Produkten 1 und 2 direkt zugeschlagen werden, da die Silos eindeutig zuordenbar sind.

3. Die Maschinenkosten sind variablen Kosten je Produktionsmenge, ebenso die Personalkosten.

4. Die Abschreibungen auf Maschinen sind linear. 
Im Planspiel findet man die Herstellungskosten A im Bereich „Rechnungswesen / Kostenrechung“. Für die Produkte 1 und 2 werden die aktuellen Werte gemäss der Planentscheidungen sowie die exakten Werte der Vorperiode dargestellt.

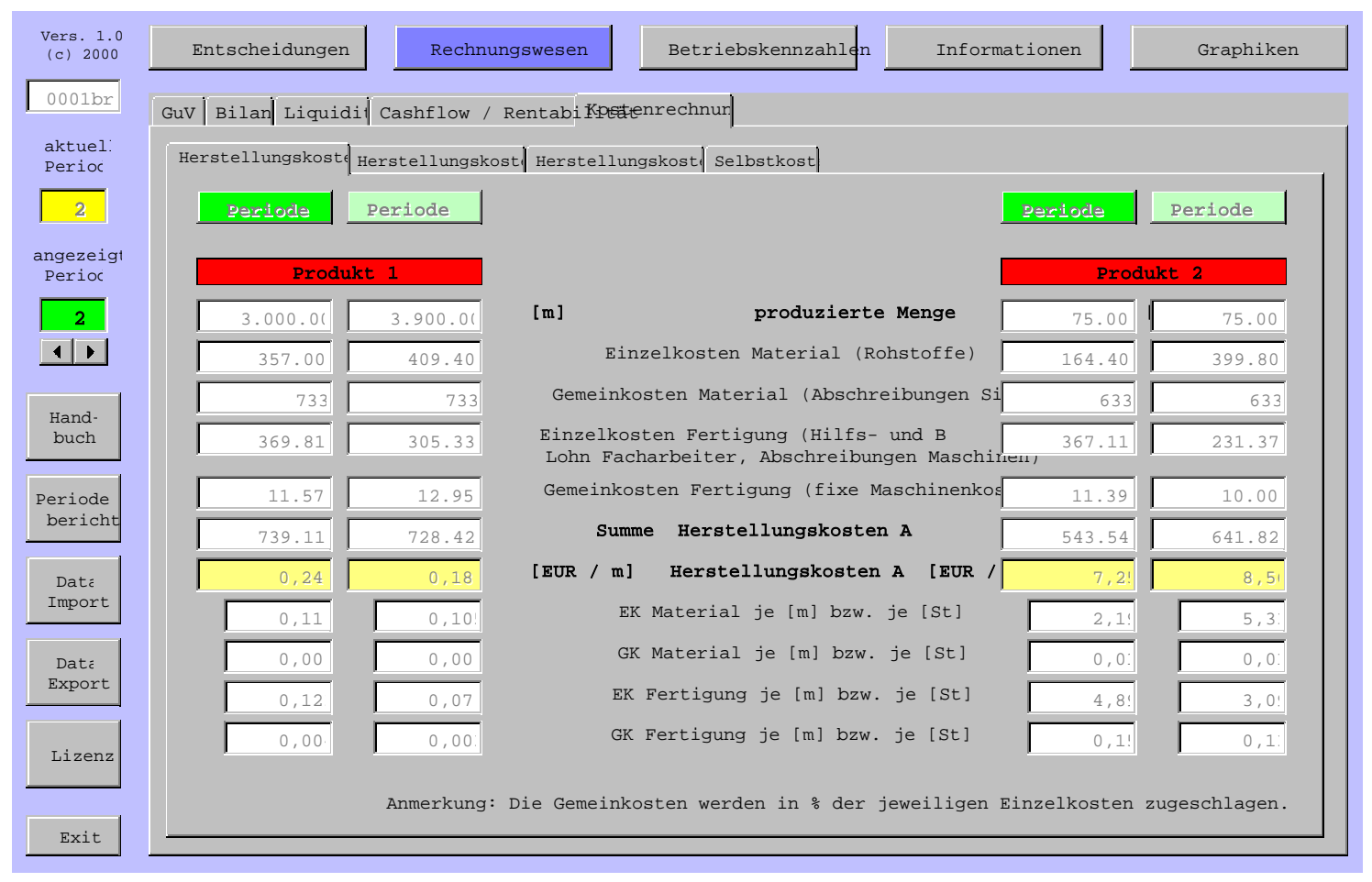

Abb. 32: Ausgabemaske Herstellungskosten A

Die Herstellungskosten werden in Summe über alle produzierten Artikel berechnet und dann je produziertem Stück ausgewiesen. Weiterhin werden die Stückkosten anteilig als Einzel- und Gemeinkosten jeweils für Material und Fertigung dargestellt. Dabei werden die Gemeinkosten in Prozent zu den relevanten Einzelkosten zugeschlagen.

Die Gemeinkosten des Materials sind in diesem Beispiel vernachlässigbar, die Silos haben im Verhältnis zu den Materialeinzelkosten zu geringe Abschreibungswerte.

\subsubsection{Kalkulation der Fertigfabrikate (Herstellungskosten B)}

Analog zur Berechnung der Herstellungskosten der beiden eigen gefertigten Halbfabrikate werden die Herstellungskosten der montierten Verkaufsartikel Singulus und Combilus wie folgt berechnet: 


\begin{tabular}{|l|c|l|}
\hline Kostenart & & Beispiel \\
\hline Einzelkosten Material & 1 & Rohstoffe, Zukaufprodukte \\
\hline Gemeinkosten Material & 2 & $\begin{array}{l}\text { Abschreibungen auf Rohstoff- und Recyclat-Silos } \\
\text { sowie auf Halbfabrikat-Lager }\end{array}$ \\
\hline Einzelkosten Fertigung & 3 & Produktion, Montage, Personalkosten Facharbeiter \\
\hline Gemeinkosten Fertigung & 4 & $\begin{array}{l}\text { Abschreibungen auf Maschinen und Läger } \\
\text { (VK-Artikel, Ausschuss), innerbetriebliche } \\
\text { Transportkosten, Personalkosten Hilfsarbeiter }\end{array}$ \\
\hline Herstellungskosten B & & \\
\hline
\end{tabular}

Tab. 33: Kalkulation der Fertigfabrikate (Herstellungskosten B)

Die Herstellungskosten der beiden Fertigfabrikate Singulus und Combilus

(Herstellungskosten B) findet man ebenfalls unter „Rechnungswesen /

Kostenrechnung“. 


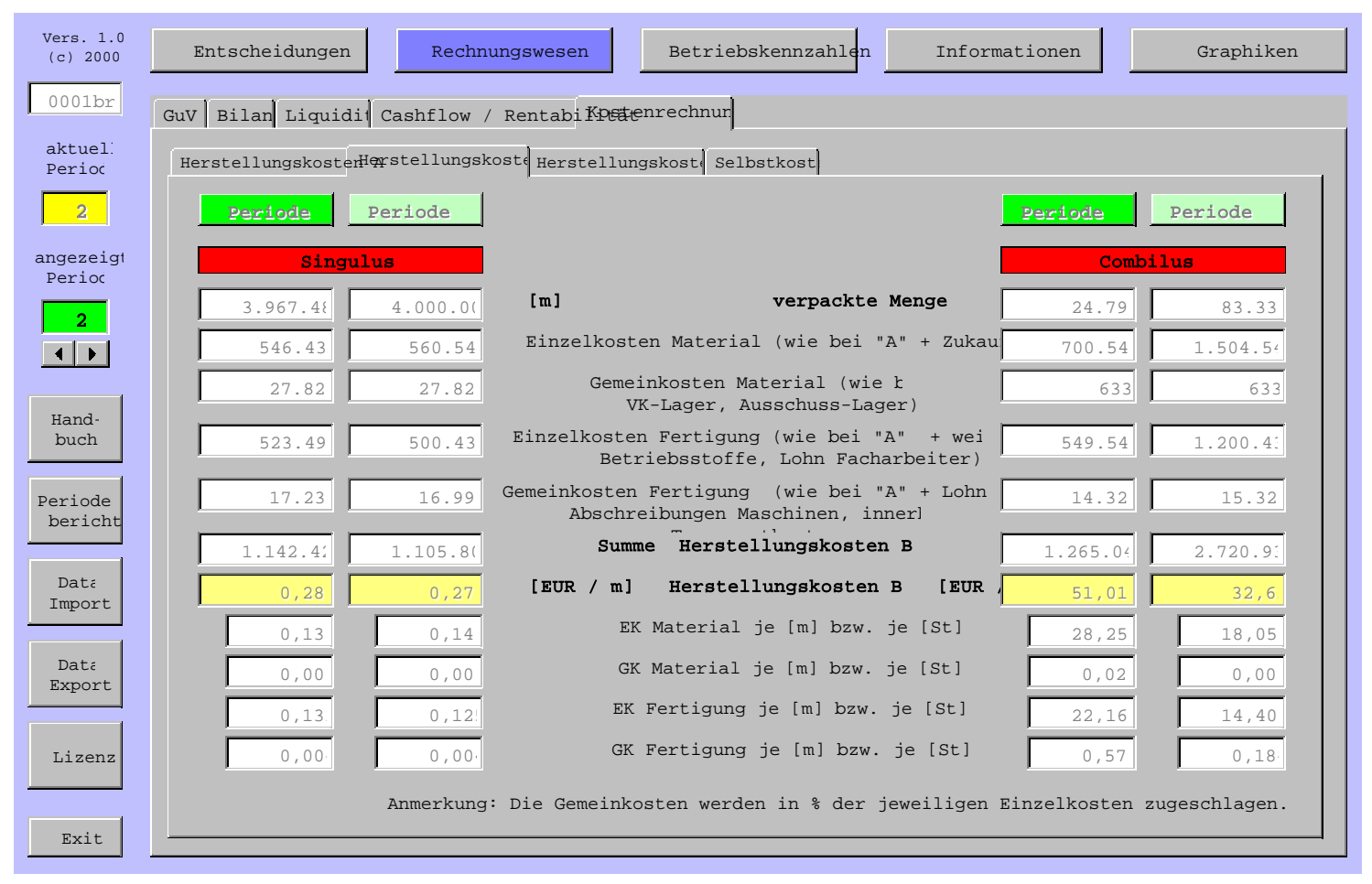

Abb. 33: Ausgabemaske Herstellungskosten B

Wie auch bei den Herstellungskosten A werden die Herstellungskosten in Stück sowie aufgeteilt nach Einzel- und Gemeinkosten als Material- und Fertigungskosten dargestellt. Auch hier werden die Gemeinkosten in Prozent der jeweiligen Einzelkosten zugeschlagen.

In diesem Beispiel ist der Einfluss der Materialgemeinkosten schon größer als bei den Herstellungskosten A, da die deutlich höheren Abschreibungen der Hallen mit eingehen. Die zugekauften Produkte 3 und 4 werden voll unter Einzelkosten Material angesetzt.

\subsubsection{Kalkulation der Verkaufsartikel (Herstellungskosten C)}

Die nachfolgende Aufstellung gilt für die Kalkulation der produzierten Artikel Singulus und Combilus. Die Herstellungskosten $\mathrm{C}$ sind die Basis für die Berechnung der Selbstkosten der VK-Artikel. Die Herstellungskosten C entsprechen im Prinzip den Herstellungskosten IV der dann gleichartigen Steuer- bzw. Handelsbilanz (siehe oben). 


\begin{tabular}{|l|r|l|}
\hline Kostenart & & Beispiel \\
\hline Einzelkosten Material & 1 & Rohstoffe, Zukaufprodukte \\
\hline Gemeinkosten Material & 2 & Abschreibungen auf Rohstoff- und Recyclat-Silos \\
\hline Einzelkosten Fertigung & 3 & Produktion, Montage, Personalkosten Facharbeiter \\
\hline Gemeinkosten Fertigung & 4 & $\begin{array}{l}\text { Abschreibungen auf Maschinen und Läger, } \\
\text { innerbetriebliche Transportkosten, } \\
\text { Personalkosten Hilfsarbeiter }\end{array}$ \\
\hline Herstellkosten B & & \\
\hline Gemeinkosten Verwaltung & 5 & Verwaltungskosten und Vorstandsgehälter \\
\hline Gemeinkosten Weiterentwicklung \\
\hline Sondergemeinkosten I & 6 & Kosten für F\&E, QM, Personalweiterbildung \\
\hline Sondergemeinkosten II & 7 & Reklamationen \\
\hline Herstellungskosten C & 8 & $\begin{array}{l}\text { Einstellungen, Entlassungen, Beratungen, } \\
\text { Anfahrtsgebühren }\end{array}$ \\
\hline Einzelkosten Vertrieb & & \\
\hline Gemeinkosten Vertrieb & 9 & LKW-Transportkosten \\
\hline Sondergemeinkosten III & 10 & Bestellgebühren für LKW, Werbung, Marktforschung \\
\hline Selbstkosten & 11 & FK-Zinsen \\
\hline & & \\
\hline
\end{tabular}

Tab. 34: Kalkulation der Verkaufsartikel (Herstellungskosten C)

\section{Anmerkungen:}

1. Die Einzelkosten Material setzen sich aus Kosten für die hergestellten Produkte 1 und 2 sowie für die Zukaufprodukte 3 und 4 zusammen.

2. Gemeinkosten Material sind die Abschreibungen auf Silos.

3. Die Einzelkosten Fertigung bestehen aus Kosten für die Fertigungsstellen. Dies sind Abschreibungen auf Produktions- und Montagemaschinen, Hilfs- und Betriebsstoffkosten sowie Personalkosten der Facharbeiter.

4. Gemeinkosten Fertigung umfassen Abschreibungen auf Lagerhallen, Ausgaben für Wartungskosten der Maschinen sowie Personalkosten der Hilfsarbeiter für die innerbetrieblichen Transporte.

5. Gemeinkosten Verwaltung sind Kosten der Verwaltung inklusive Vorstandsgehälter. Diese Verwaltungskosten werden im Gegensatz zur

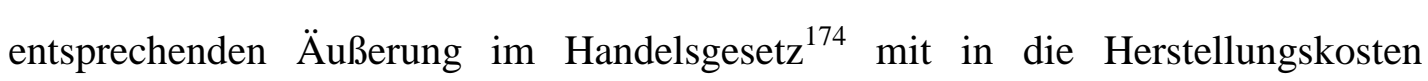
aufgenommen.

6. Gemeinkosten Weiterentwicklung sind Aufwendungen für Forschung und Entwicklung, für Qualitätsmanagement und für Weiterbildung von Personal. 
7. Sondergemeinkosten I sind Kosten für Reklamationen. Diese fallen nach drei Perioden in Abhängigkeit des damaligen Absatzes sowie der damaligen Unterstützungsfaktoren „Qualitätsmanagementsystem“ und „Aufwand für Wartung“ an.

8. Sondergemeinkosten II bestehen aus Kosten für die Einstellung und Entlassung von Mitarbeitern sowie aus gegebenenfalls anfallenden Kosten für Beratungsgebühren durch die Spielleitung. Weiterhin werden hier die gegebenenfalls anfallenden Anfahrtskosten für Rohstoffe und Zukaufprodukte aufgenommen.

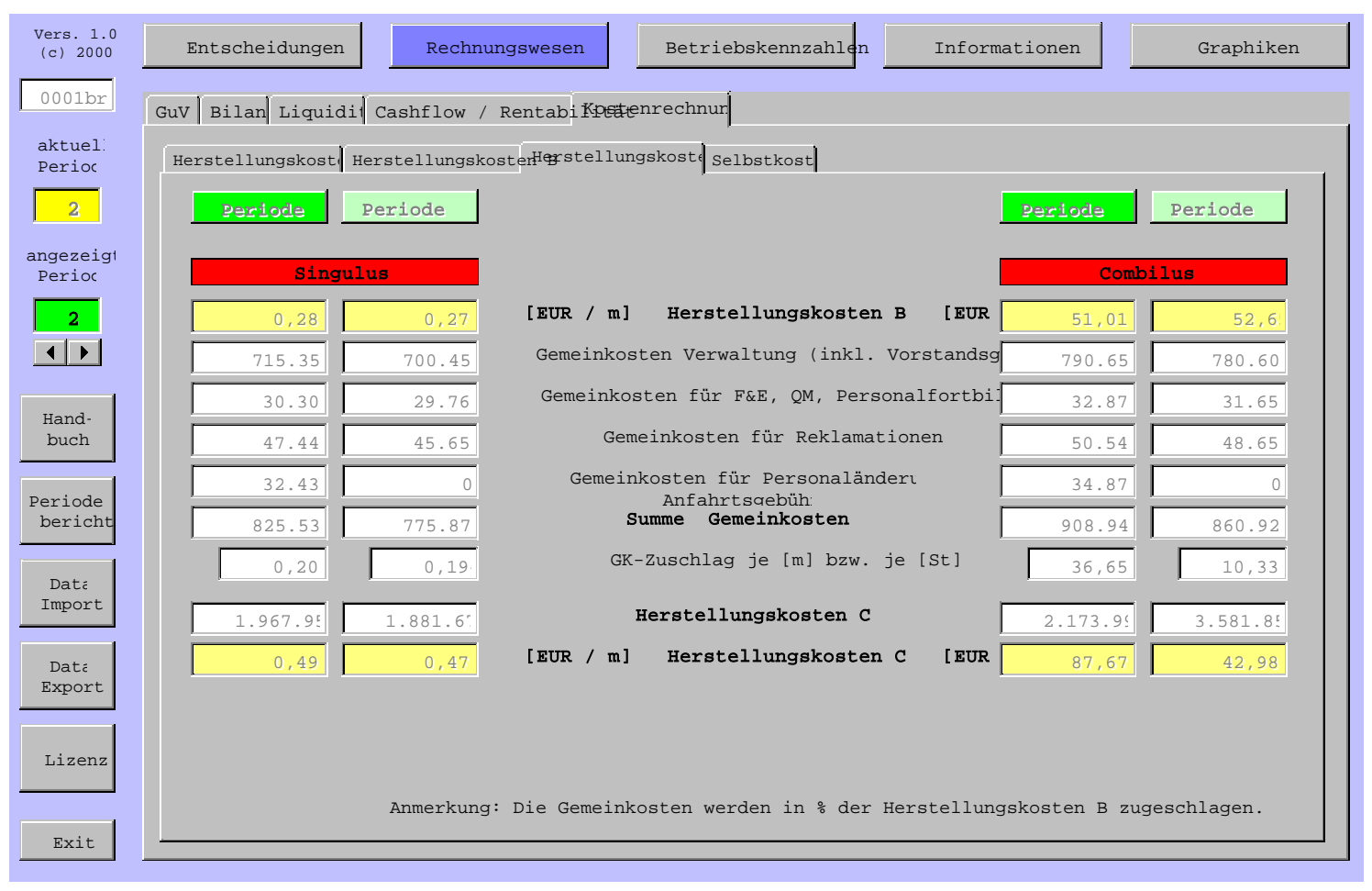

Abb. 34: Ausgabemaske Herstellungskosten C

Die Herstellungskosten $\mathbf{C}$ errechnen sich aus den noch ausstehenden Gemeinkosten, die nicht den Produkten Singulus und Combilus direkt zugeschlagen werden können (nicht produktspezifische Gemeinkosten). Diese Gemeinkosten werden in Prozent der absoluten Herstellungskosten B zugeschlagen, d.h. bei Herstellungskosten B von 100 für den Artikel Singulus und 200 für den Artikel Combilus teilen sich die Gemeinkosten der Verwaltung im Verhältnis 1:2 auf die beiden Produkte auf. 


\subsubsection{Kalkulation der Verkaufsartikel (Selbstkosten)}

Um die für die Preisbestimmung wichtigen Selbstkosten zu ermitteln müssen die noch nicht erfasst Kosten auf die Herstellungskosten C verteilt werden. Die Selbstkosten werden abermals in Summe und je verpackter Menge errechnet.

\begin{tabular}{|l|c|l|}
\hline Kostenart & & Beispiel \\
\hline Herstellungskosten C & & \\
\hline Einzelkosten Vertrieb & 1 & LKW-Transportkosten \\
\hline Gemeinkosten Vertrieb & 2 & Bestellgebühren für LKW, Werbung, Marktforschung \\
\hline Sondergemeinkosten III & 3 & FK-Zinsen \\
\hline Selbstkosten & & \\
\hline
\end{tabular}

Tab. 35: Kalkulation der Verkaufsartikel (Selbstkosten)

\section{Anmerkungen:}

1. Einzelkosten Vertrieb sind die variablen Kosten für den Gütertransport. Dabei fallen nur für die physisch transportierten Mengen Kosten an: Entfernungskosten (Inland, Export, Ausschreibung) sowie Verpackungsgebühr (Palette) je $\mathrm{m}^{3}$.

2. Gemeinkosten Vertrieb sind Bestellgebühren für die LKW sowie Aufwendungen für Werbung und Marktforschung. Die Bestellgebühren sind abhängig von der Anzahl der bestellten LKW und unabhängig von der letztendlich benutzten Anzahl.

3. Sondergemeinkosten III sind Aufwendungen für Fremdkapitalzinsen. 


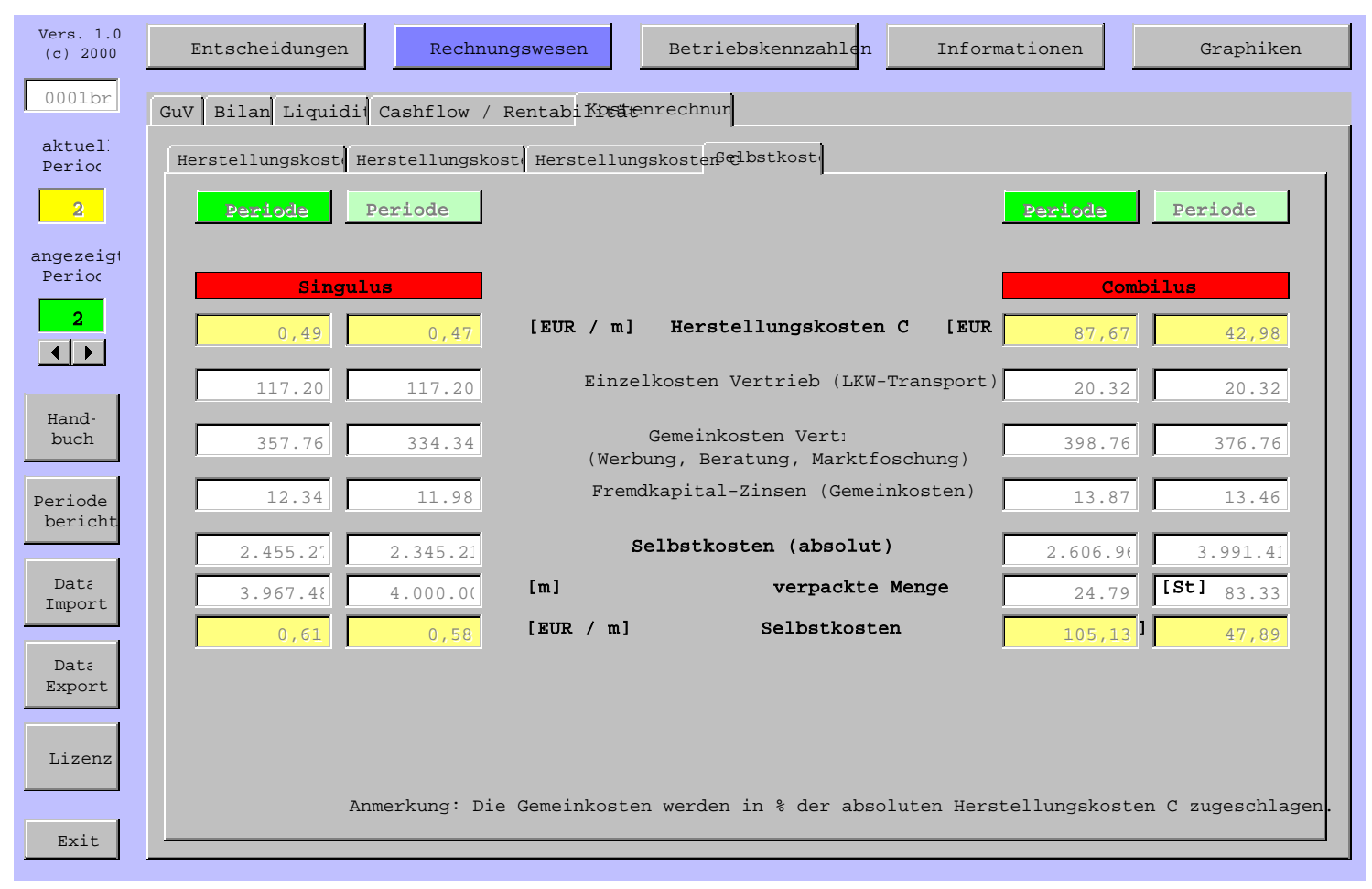

Abb. 35: Ausgabemaske Selbstkosten

Die Selbstkosten dienen zur Kalkulation der Verkaufspreise, es sind alle Kosten erfasst, die für den Absatz der Produkte anfallen. Wiederrum werden die Kosten in Summe sowie im Verhältnis zu der produzierten Menge angegeben. Produkte, die aufgrund technischer Mängel ausgesondert werden, sind nicht auf diese Kosten umgelegt. D.h., würde der Verkaufspreis genau dem Selbstkostenpreis entsprechen, würde voraussichtlich ein Verlust anfallen, da 100 Prozent der hergestellten Produkte nicht in den Verkauf gelangen.

Die Zeitreihe der Entwicklung der verschiedenen (Ist-) Herstellungs- und Selbstkosten werden nach einer simulierten Periode in einer Graphikdatei im Verzeichnis GRAPHICS hinterlegt. 


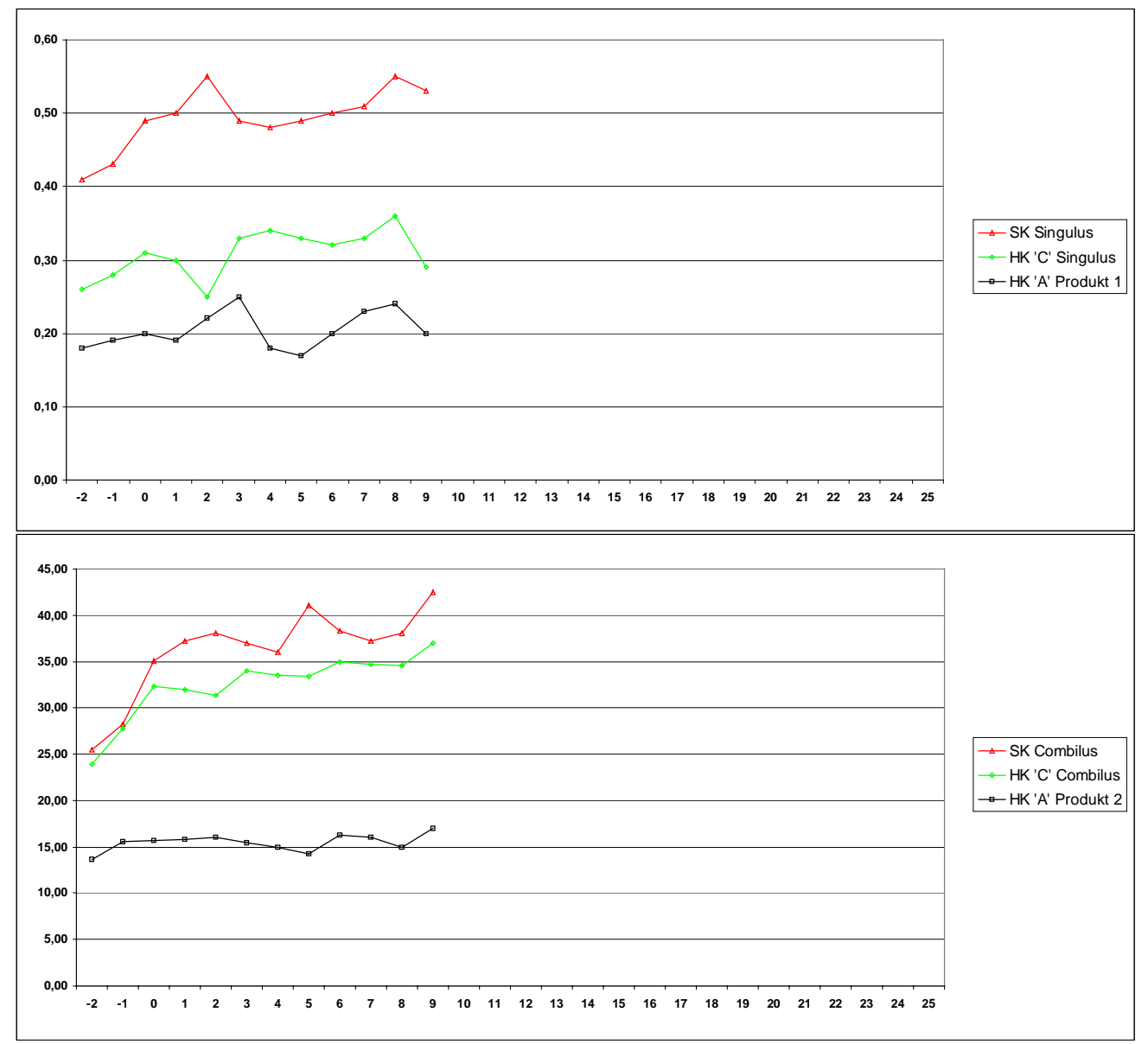

Abb. 36: Kennzahlen Produktkalkulation (Herstellungskosten)

Diese Darstellung ist bewusst programmiert worden, da sie einen guten Einblick über die Entwicklung der Herstellungs- und Selbstkosten im Zeitverlauf gibt. Für die Analyse jedes einzelnen Teams zum Ende des Planspiels ist eine solche Aufstellung sehr hilfreich. Sehr leicht können die Zusammenhänge bzw. Auswirkungen der Entscheidungen dargestellt werden. 


\subsubsection{Gewinn- und Verlustrechnung}

Die GuV orientiert sich im Planspiel an den US-amerikanischen Grundsätzen:

\begin{tabular}{|lll|}
\hline & Umsatzerlöse & 1 \\
$=$ & Umsatzkosten & 2 \\
\hline- & Bruttoergebnis vom Umsatz & \\
& Vertriebskosten, Allgemeine Verwaltungskosten, Sonstige betriebliche & 3 \\
- & Aufwendungen & \\
$+/-$ & F\&E-, QM- und Personalweiterbildungskosten (,Zukunftskosten“) & 4 \\
$=$ & Sonstige betriebliche Erträge & 5 \\
\hline$+/-$ & Finanzergebnis & 6 \\
$=$ & Ergebnis vor Ertragsteuern & \\
\hline- & Verlustvortrag & \\
$=$ & zu versteuerndes Ergebnis & 8 \\
\hline- & Steuern vom Einkommen und vom Ertrag & \\
$=$ & Ergebnis nach Steuern & \\
\hline
\end{tabular}

Tab. 36: Gliederung der GuV $\sqrt{175}$

Der Übersicht halber werden die einzelnen Posten kurz erläutert:

1. Umsatzerlöse sind Erlöse aus verkauften Produkten Singulus und Combilus. Dabei werden die fakturierten Umsätze ausgewiesen, die nicht zwingend zu Einzahlungen führen. Erlöse aus Vermietung oder Verkauf von Produktionsanlagen werden hier nicht summiert.

2. Umsatzkosten sind Kosten für Beschaffung Rohstoffe 1 und 2, Beschaffung Halbfabrikate 3 und 4, Kosten der Fertigungsstellen Produktion und Montage der Produkte und Artikel, Personalkosten der Facharbeiter, Abschreibungen auf Silos, Lager und Maschinen, Kosten für Wartungsaufwendungen und Kosten für innerbetriebliche Transporte. Die Beschaffungskosten für Rohstoffe und Halbfabrikate stammen zu 100 Prozent aus der aktuellen Periode.

3. Vertriebskosten umfassen Transportkosten, Aufwand für Werbung Inland und Export sowie Kosten für Marktforschung. ${ }^{176}$ Allgemeine Verwaltungskosten sind Kosten der Verwaltung, Vorstandsgehälter und Kosten für Beratungsleistungen

175 Vgl. umfassend KPMG (Rechnungslegung, 1999).

176 Nicht enthalten sind die üblicherweise ebenfalls $\mathrm{zu}$ den Vertriebskosten $\mathrm{zu}$ rechnenden Kosten für Verkaufsverpackungen oder Kosten der Versandverpackung. Die Kosten des Verpackungsvorganges werden bereits bei der Montage erfasst. Vgl. Wundrack (Vertriebskostenrechnung, 2000). 
durch die Spielleitung. Die sonstigen betrieblichen Aufwendungen setzen sich z.B. aus dem Kauf von Maschinen, Gabelstaplern, Silos und Lagerflächen zusammen. Weiterhin werden hier die Kosten für Personaleintellungen und entlassungen gebucht.

4. Zukunftskosten sind die Summe der Aufwendungen für F\&E, QM-System und Personalweiterbildung.

5. Sonstige betriebliche Erträge beinhalten Erträge aus Vermietung Silos, Lager oder Gabelstapler.

6. Finanzergebnis sind Aufwendungen für Zinsverpflichtungen sowie Erträge aus Wertpapieren.

7. Verlustvortrag ist ein eventuell in der Vorperiode angefallener Verlust, der mit den (hoffentlich) erzielten Gewinnen der aktuellen Periode verrechnet wird.

8. Steuern berechnen sich anhand des anfallenden Gewinns. Ist der Gewinn negativ, werden keine Steuern bezahlt. Dafür wird der ausgewiesene Gewinn als Verlustvortrag in die nächste Periode übernommen. 


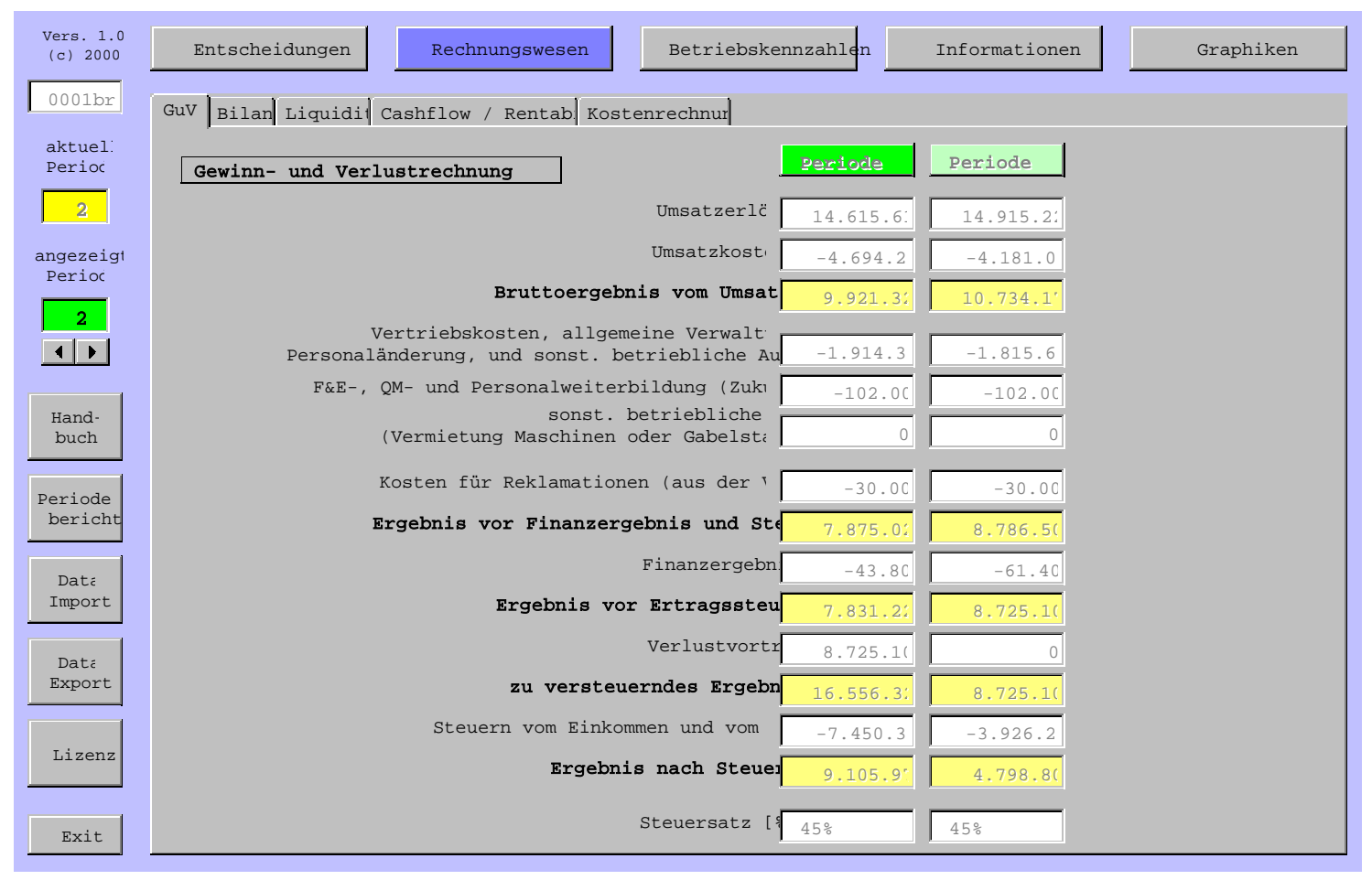

Abb. 37: Ausgabemaske GuV

In der obigen Maske werden erstmalig keine Soll- und Ist-Werte dargestellt. Die GuV richtet sich allein nach den Ist-Werten. Solange man in der Planungsphase ist - eine Simulation durch die Spielleitung hat noch nicht stattgefunden - werden die Ist-Werte genommen, die sich nach der internen Simulation der Soll-Werte ergeben. 177

Das Finanzergebnis ist in obigem Beispiel zweimal negativ. Das bedeutet, dass mehr Kreditzinsen als Wertepapiererträge angefallen sind. Der Steuersatz beträgt in diesem Beispiel 45 Prozent, er ist durch die Spielleitung anpassbar.

177 Im Prinzip sind alle Werte unterhalb der im Original kräftig grün, hier mit Periode 2 dargestellten linken Spalte Werte, die bisher rein durch die interne Simulation errechnet wurden. Sobald eine Periode abgeschlossen ist, ist sie ,vergangen“" (und nicht mehr aktuell). 
Auszüge der GuV werden im Verzeichnis GRAPHICS graphisch dargestellt. Wie schon bei der Kostenrechnung eignet sich eine solche Darstellung insbesondere für regelmäßige Auswertungen der Entscheidungen und deren Auswirkungen.

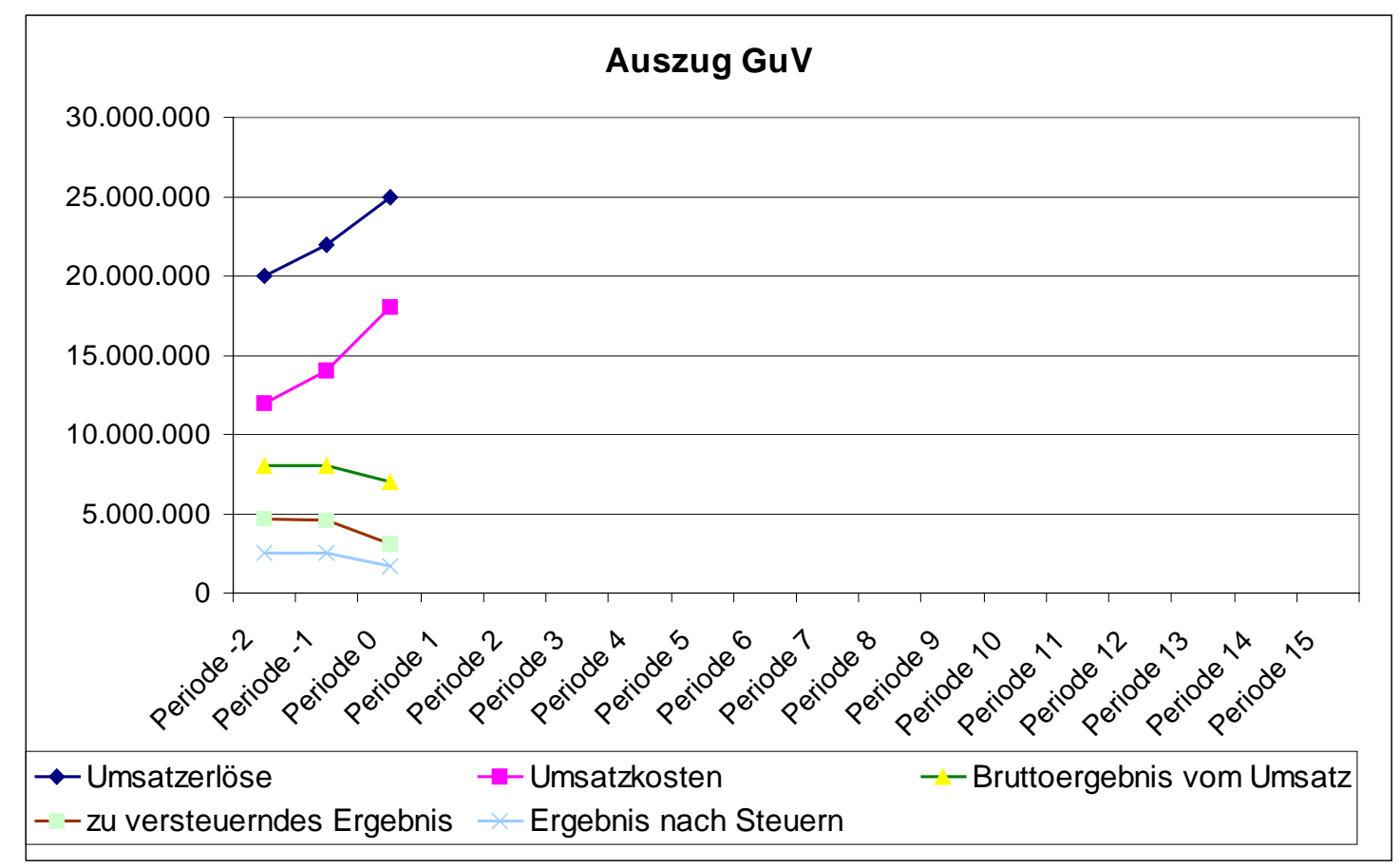

Abb. 38: Ausgewählte Kennzahlen GuV

Es werden für obige Darstellung fünf Kennzahlen der GuV ausgewählt: Umsatzerlöse, Umsatzkosten, das Bruttoergebnis vom Umsatz (als Subtraktion der beiden vorherigen Größen), das zu versteuernde Ergebnis sowie das Ergebnis nach Steuern. Diese werden im Zeitverlauf ab zwei Perioden vor dem eigentlichen Start des Planspiels dargestellt. 


\subsubsection{Bilanz}

Folgendes Bilanzschema wird im Planspiel verwendet:

\begin{tabular}{|lr|lr|}
\hline AKTIVA & & PASSIVA \\
\hline Anlagevermögen & & Eigenkapital \\
Gebäude & 1 & Gezeichnetes Kapital & 11 \\
Produktionsmaschinen & 2 & Gewinnvortrag & 12 \\
Fahrzeuge & 3 & & \\
& & Fremdkapital & \\
Umlaufvermögen & & Langfristige Verbindlichkeiten & 13 \\
Rohstoffe / Recyclat & 4 & Verbindlichkeiten aus Lieferungen & 14 \\
Halbfabrikate & 5 & Liquiditätskredit & 15 \\
Fertigfabrikate & 6 & Rückstellungen & 16 \\
Abfall & 7 & & \\
Wertpapiere & 8 & & 17 \\
Forderungen & 9 & & \\
Kasse & 10 & Periodengewinn & \\
\hline Summe Aktiva & & Summe Passiva & \\
\hline
\end{tabular}

Tab. 37: Gliederung der Bilanz

\section{Anmerkungen:}

1. Gebäude sind Lagerhallen und Silos. Lagerhallen werden über 50 Jahre, Silos über 10 Jahre linear abgeschrieben und zum Restwert bewertet.

2. Produktionsmaschinen bestehen aus zwei unterschiedlichen Produktionsmaschinentypen und einem Montagemaschinetyp. Sie werden über 10 Jahre abgeschrieben und nach der Abschreibungsdauer gegen Rechnung entsorgt.

3. Fahrzeuge sind Gabelstapler, die über 5 Jahre abgeschrieben werden. Nach Ende der Abschreibungsdauer werden sie gegen Gebühren aus dem Bestand entfernt.

4. Rohstoffe werden zu Einkaufspreisen bewertet. Mit anderen Worten: Einem eventuellen Zahlungsziel gegenüber einem Lieferanten steht hier ein höherer als wirklich bezahlter Betrag gegenüber. Das Recyclat wird gemäß den anfallenden Kosten für die Aufbereitung bewertet.

5. Halbfabrikate werden zu Herstellungskosten bewertet. Sie dazu Kapitel 4.4.6 Kostenrechnung.

6. Fertigfabrikate werden ebenfalls zu Herstellungskosten bewertet. 
7. Abfall oder Ausschuss wird mit 10 Prozent der Herstellkosten bewertet. Dabei werden Rückstellungen für die Entsorgungsgebühren gebildet.

8. Wertpapiere sind der Bestand an eigenen Wertpapieren. Sie werden zu Durchschnittspreisen bewertet.

9. Forderungen kommen aus Zahlungszielen durch Lieferanten zustande. Sie betragen anfangs 30 Prozent der Rechnungssumme und gelten für die Rohstofflieferanten ebenso wie für die Zulieferer der Halbfabrikate 3 und 4.

10. Kasse ist die Summe der Ein- und Auszahlungen der Periode. Im Liquiditätsbericht wird hierauf genau eingegangen.

11. Gezeichnetes Kapital ist das eingezahlte (Stamm-) Kapital der Firma. Es ist für alle fünf beteiligten Unternehmen gleich hoch.

12. Gewinnvortrag entspricht dem Posten „Periodengewinn“ der Vorperiode plus dem Gewinnvortrag der Vorperiode.

13. Langfristige Verbindlichkeiten stehen für Kredite von Banken. Die maximal verfügbaren langfristigen Verbindlichkeiten sind beschränkt.

14. Forderungen aus Lieferungen und Leistungen sind unbezahlte Rechnungen aus der aktuellen Periode. Der Anteil beträgt anfangs 20 Prozent des fakturierten Umsatzes.

15. Liquiditätskredit wird - soweit notwendig - automatisch berechnet und ausgewiesen. Es fallen hierbei höhere Zinsen wie bei einem regulären Kredit an.

16. Rückstellungen werden für die Entsorgungskosten des Ausschusses gebildet. Diese werden in der folgenden Periode aufgelöst und in der Liquiditätsrechnung als Kasseneingang verbucht.

17. Periodengewinn errechnet sich aus Summe Aktiva minus Summe Eigenkapital minus Summe Fremdkapital.

178 Hier ist zu beachten, dass der Einsatz von Recyclat eine höhere Ausschussquote zur Folge hat. Dadurch können die günstigeren Materialkosten gegebenenfalls durch höhere Produktionskosten aufgebraucht werden. 


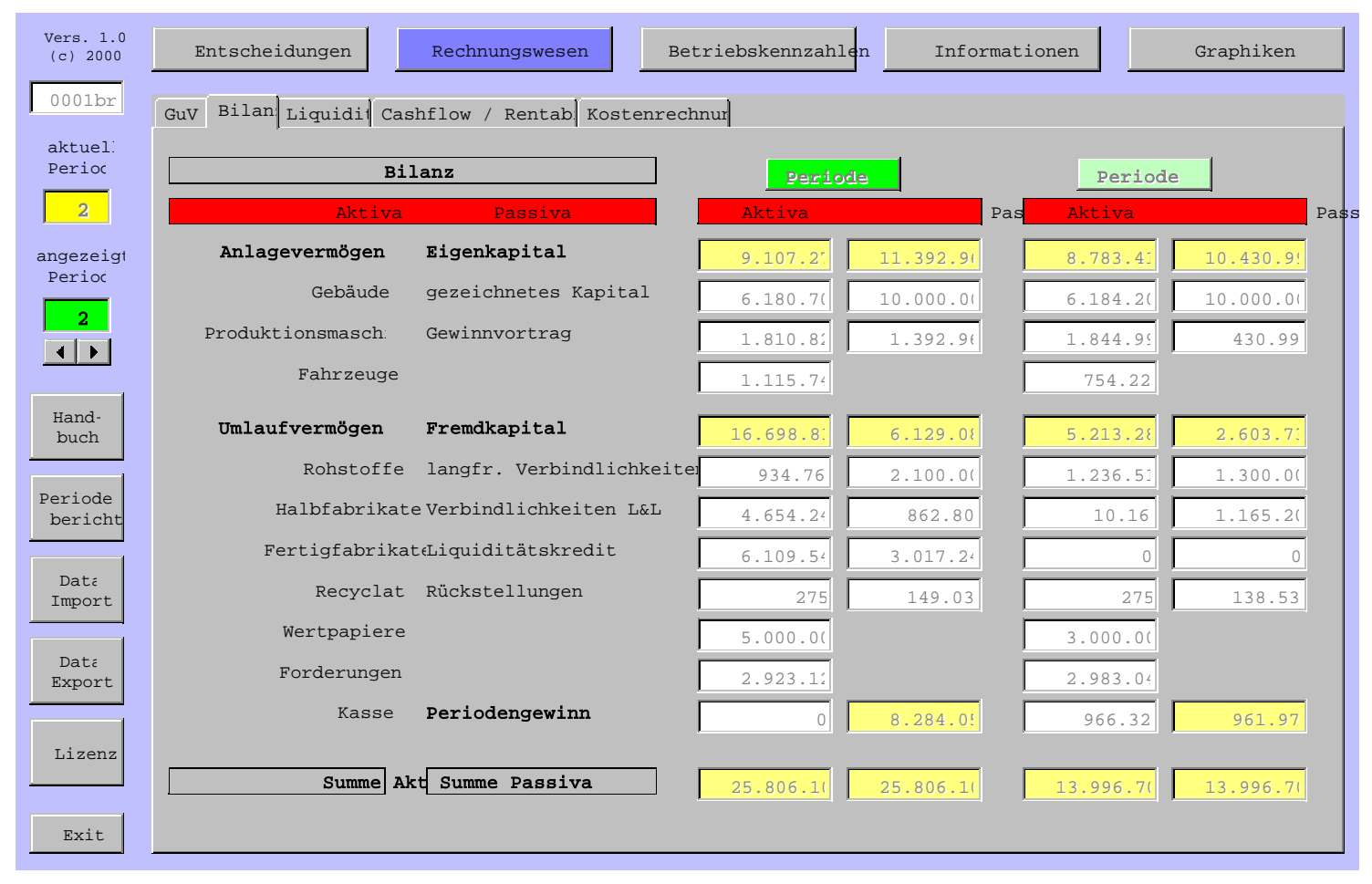

Abb. 39: Ausgabemaske Bilanz

Das Eigenkapital berechnet sich aus dem gezeichneten Kapital - dieser Wert ist durch die Spieler nicht beeinflussbar und durch die Spielleitung vorgegeben - und dem Gewinnvortrag plus dem Periodengewinn jeweils aus der vorhergehenden Periode.

Im Verzeichnis GRAPHICS werden nach jeder Periode ausgewählte Bilanzkennzahlen dargestellt. 


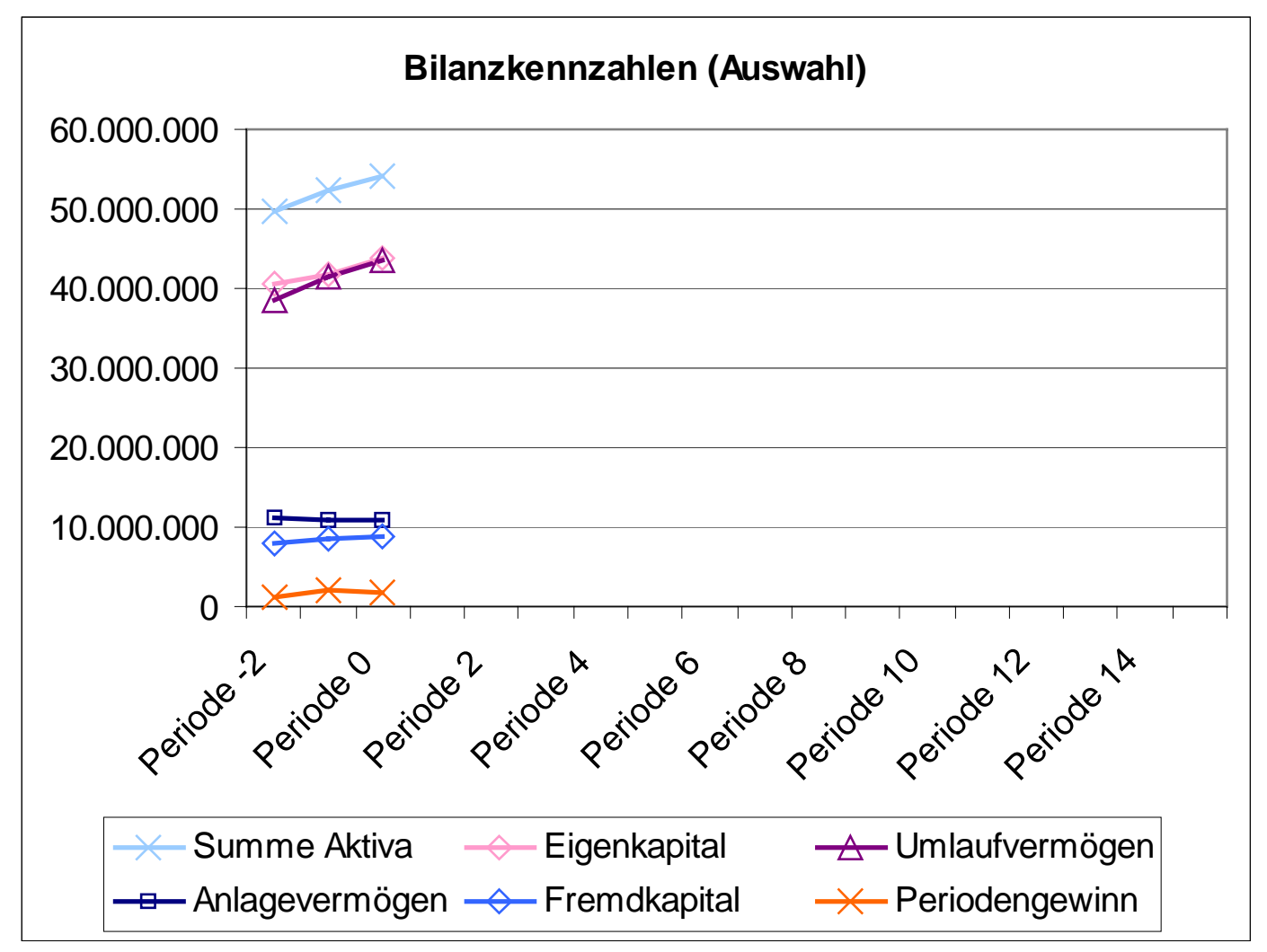

Abb. 40: Ausgewählte Bilanzkennzahlen

Man erhält einen guten und schnellen Überblick über die Entwicklung wichtiger Bilanzkennzahlen. In obigem Beispiel entwickeln sich die Aktiva (und damit natürlich auch die Passiva) aufgrund einer starken Zunahme des Umlaufvermögens. Bei nahezu gleichbleibenden Gewinn steigt somit auch das Eigenkapital in ähnlichem Maße an.

Weiterhin wird die Aufteilung des Anlagevermögens dargestellt. Hierbei wird immer nur eine Periode dargestellt. 


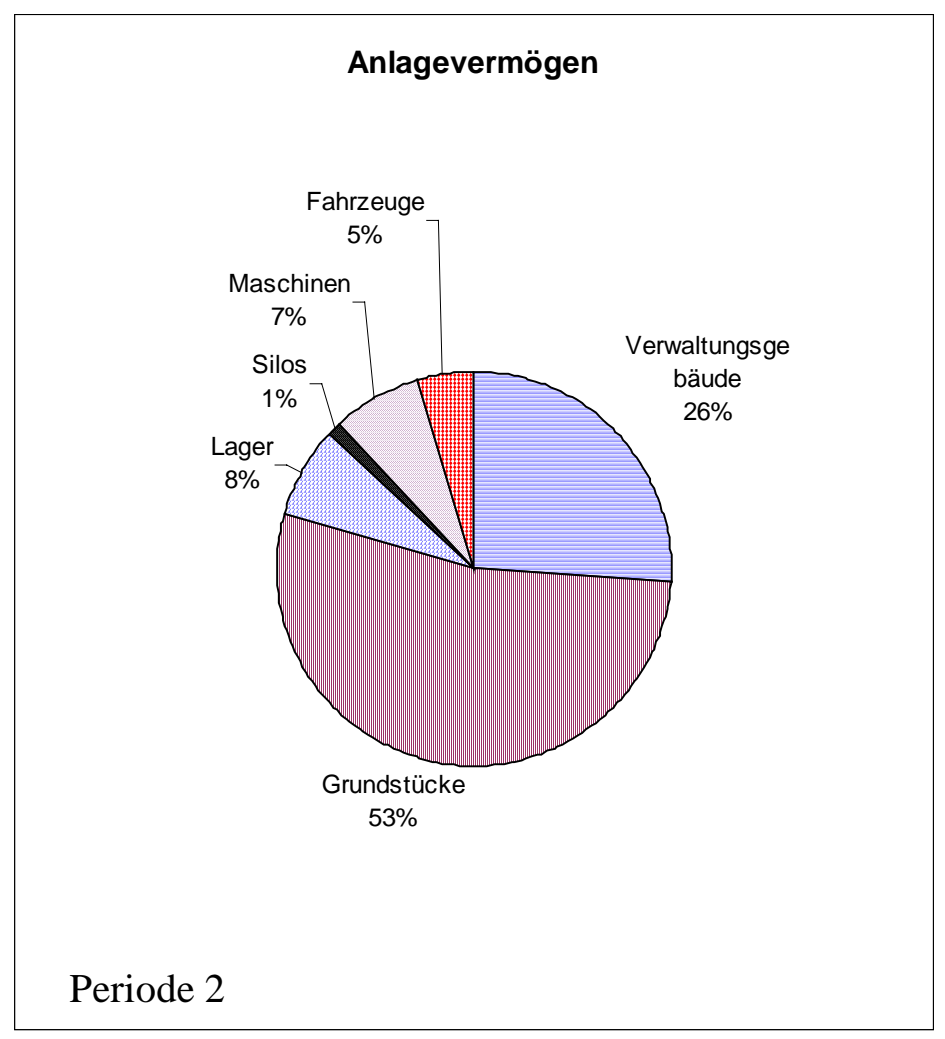

Abb. 41: Kennzahlen Anlagevermögen

Sehr gut erkennbar ist der relativ große Vermögensanteil an Grundstücken und Verwaltungsgebäuden. Auf den ersten Blick erscheint das Unternehmen sehr wertvolle bzw. eine sehr große Anzahl von Grundstücken und Verwaltungsgebäuden zu besitzen. $\mathrm{Ob}$ dies zur Zielereichung wirklich notwendig ist gilt es durch jedes Team zu analysieren.

Mit dem folgenden Bild wird das Umlaufvermögen detaillierter dargestellt. Oben links ist die Verteilung des Umlaufvermögens in Rohstoffe, Halbfabrikate, VK-Artikel und Recyclat dargestellt. Auf den restlichen drei Bildern werden VK-Artikel, Halbfabrikate und Rohstoffe auf deren einzelne Bestandteile herunter gebrochen. 

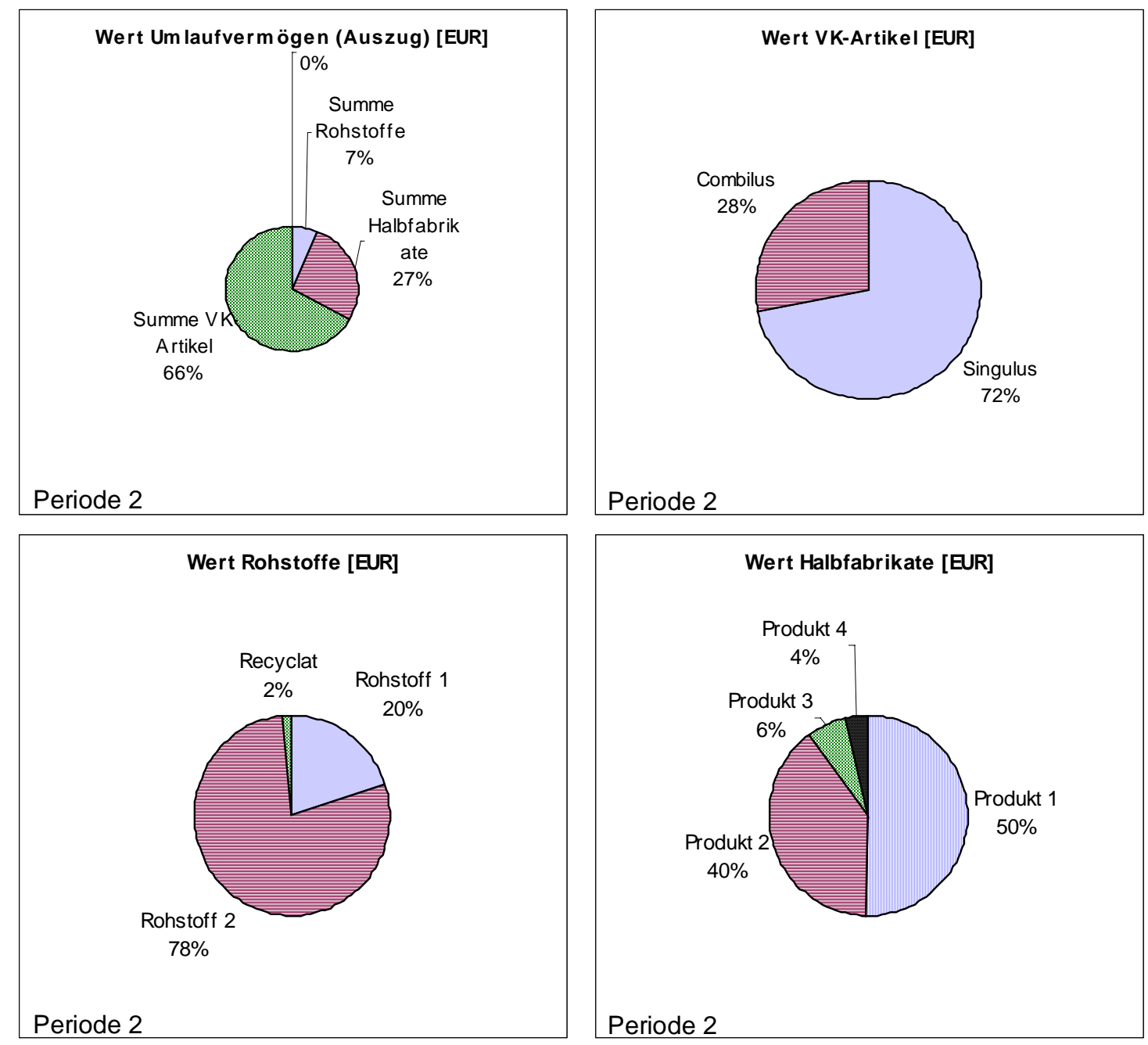

Abb. 42: Kennzahlen Umlaufvermögen

Aus der oben dargestellten Abbildung lassen sich verschiedene Interpretationen ablesen. So könnte dies heißen, dass Singulus derzeit überdurchschnittlich im VK-Lager liegt, weil der Absatz nicht wie geplant realisiert werden kann. Oder aber dass Singulus in der letzten Stufe (Verpackung) einen hohen Wertzuwachs erhält.

Insgesamt lässt sich allein aufgrund der obigen Abbildungen jedoch keine abschließende Aussage treffen. Die Auswertungen repräsentieren nur einen Ausschnitt der tatsächlichen Lage des Unternehmens. Diese zu erhalten und daraus die richtigen Entscheidungen für das Unternehmen zu treffen ist die Aufgabe der Teams. 


\subsubsection{Liquiditätsrechnung}

Die Liquiditätsrechnung stellt die Einzahlungen den Auszahlungen in einer Periode gegenüber. Hierbei wird das eigene Zahlungsverhalten bei Rechnungen der Rohstoffe und Halbfabrikate und das Zahlungsverhalten der Kunden mit berücksichtigt. Sollten der Anfangskassenbestand und die Einzahlungen für die Auszahlungen nicht ausreichen, steht ein Liquiditätskredit bereit.

\begin{tabular}{|ll|}
\hline Kassenanfangsbestand & \\
\hline Einzahlungen & Einzahlungen aus Verkaufserlösen Ist-Periode \\
& Einzahlungen aus Verkaufserlösen Vor-Periode \\
& Zinsen und Sondererträge (Maschinenverkäufe, Mieten) \\
& Kreditaufnahme / Auflösung von Rückstellungen \\
\hline Auszahlungen & Lohn \\
& Vertriebskosten / Kosten der Verwaltung (inkl. Vorstand) \\
& Auszahlungen für Lieferungen Ist-Periode \\
& Auszahlungen für Lieferungen Vor-Periode \\
& Investitionen für Silos, Hallen, Maschinen, Gabelstapler \\
& Werbung, F\&E, QM-System, Personalfortbildung \\
& Personaländerungskosten, FK-Zinsen, Beratungsgebühren \\
& Auszahlungen Reklamationen \\
& Kauf Wertpapiere / Rückzahlung Kredite \\
& Steuern \\
\hline Zwischenstand & \\
\hline Einzahlung & Liquiditätskredit \\
\hline Kassenendbestand & \\
\hline
\end{tabular}

Tab. 38: Liquiditätsrechnung

Die Einzahlungen werden in „Einzahlungen aus Verkaufserlösen Ist-Periode“ und in „Einzahlungen aus Verkaufserlösen Vor-Periode“ unterschieden. Durch die Spielleitung kann das Zahlungsverhalten der Kunden beeinflusst werden. Lautet das Verhältnis z.B. 80:20, so werden 80 Prozent der fakturierten Rechnungen von den Kunden in der aktuellen Periode bezahlt. Die restlichen 20 Prozent folgen in der nächsten Periode.

Der eventuell nötige Liquiditätskredit wird automatisch durch das Computerprogramm errechnet, dem Spieler durch ein Fenster mitgeteilt und die Kasse damit ausgeglichen. Für diesen Liquiditätskredit ist ein höherer Zins als bei einem geplanten Zins zu bezahlen. Dieser Kredit wird automatisch zu Beginn einer neuen Periode wieder auf Null gesetzt. 


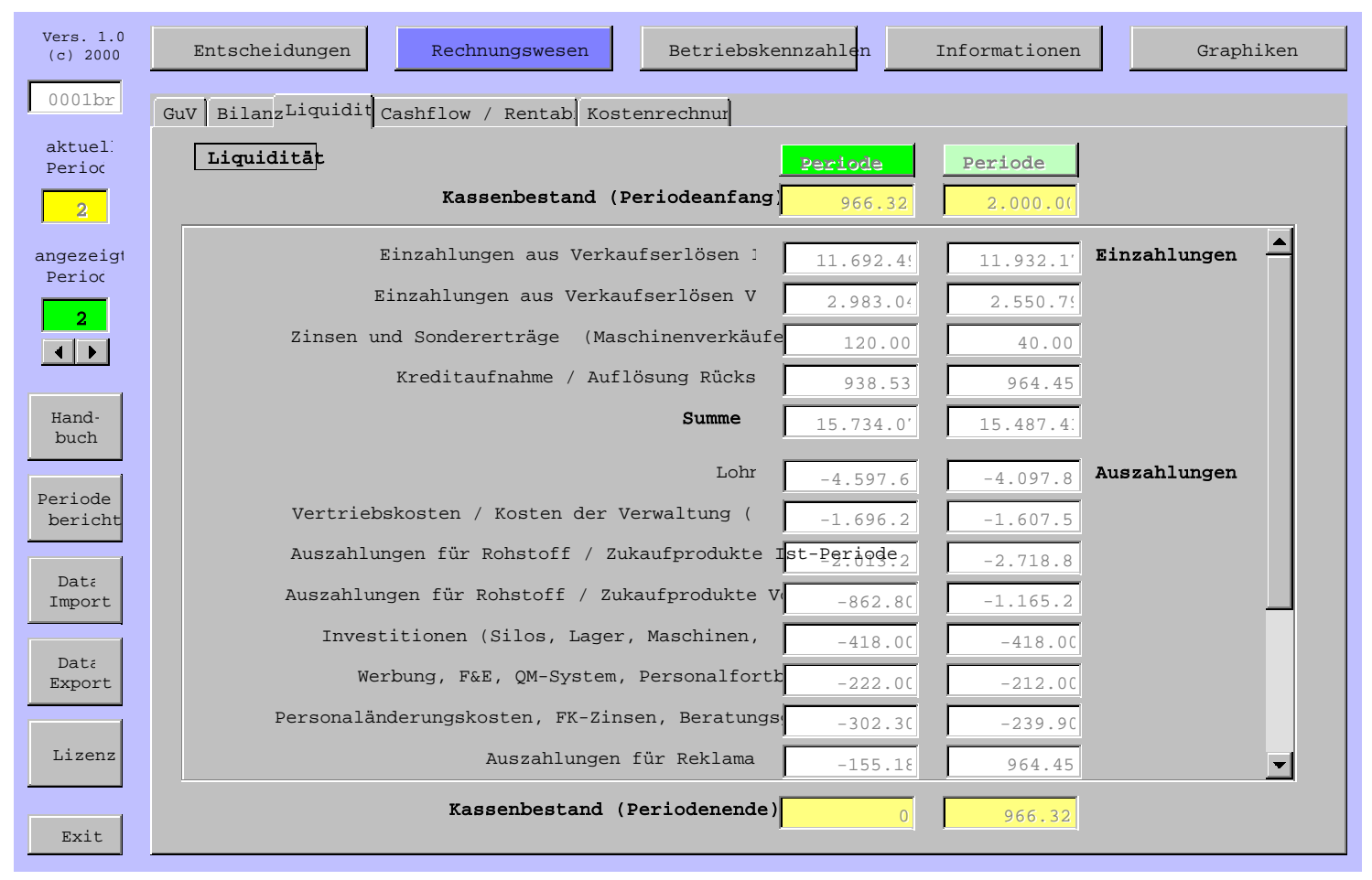

Abb. 43: Ausgabemaske Liquidität

Aufgrund der Größe der Computerbildschirme und der getroffenen Unterteilung der Ein- und Auszahlungen kann in diesem Falle nicht die gesamte Aufstellung der liquiditätswirksamen Ein- und Auszahlungen erfolgen. In der obigen Bildschirmmaske folgen nach Auszahlungen für Reklamationen noch die Auszahlungen „Kauf Wertpapiere, Rückzahlung Kredite“, „Steuern“, „Summe Auszahlungen“, „Zwischensumme Kasse“ sowie „Liquiditätskredit“. In der Liquiditätsrechnung ist aufgrund der gewählten Gruppen eine sehr gute Übersicht über die Zahlungsvorgänge sichtbar. So sieht man z.B. sofort, wie hoch die Auszahlungen für Lohn, Rohstoffe oder Reklamationen waren. Eine detailliertere Aufstellung nach den einzelnen Kosten findet man an anderer Stelle (Menü Betriebskennzahlen). 
Die Aus- und Einzahlungen werden wiederum im Verzeichnis GRAPHICS dargestellt.

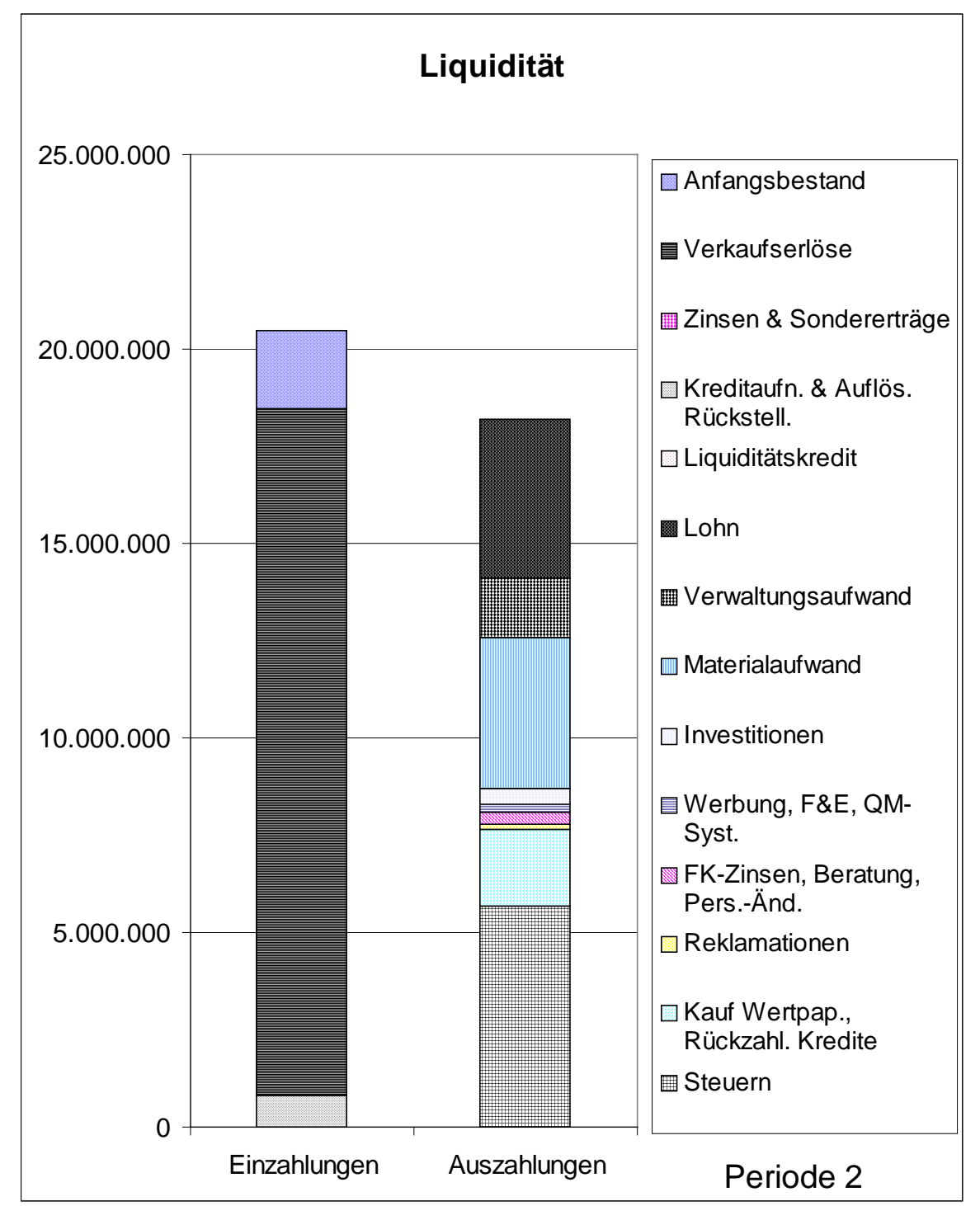

Abb. 44: Liquidität

Auch in diesem Fall werden wiederum nur die aktuellen Periodenwerte dargestellt. Man erhält einen guten Überblick über die Auszahlen und kann dabei die Kostenreiber schnell identifizieren. In diesem Schaubild werden die Einzahlungen nicht in Einzahlungen aus der vergangenen Periode und Einzahlungen aus der laufenden Periode unterschieden. 


\subsubsection{Finanzkennzahlen}

Die Kennzahl Cashflow wird insbesondere für zwei unterschiedliche Verwendungsrichtungen herangezogen: Zum einen zur Analyse der Finanzkraft, zum anderen zur Analyse der Ertragskraft eines Unternehmens. Der Cashflow kann vereinfacht als liquiditätswirksamer Jahreüberschuss, als Zahlungsüberschuss aus dem laufenden Betriebsprozess, als Teil des Umsatzüberschusses, der nicht in der gleichen Periode zu Ausgaben oder Einnahmen geführt hat, als Kapitalrückfluss aus dem Unternehmensprozess oder als Finanzmittelzufluss bezeichnet werden. 179

In der Regel geht man beim Cashflow von der Basisgröße Bilanzgewinn aus, die sich in der Erfolgsrechnung als Differenz von Erträgen und Aufwendungen ergibt. Man zählt alle Aufwendungen hinzu und zieht alle Erträge wieder ab, die in dem betrachteten Zeitraum nicht finanzwirksam gewesen sind. 180 Aufgrund unterschiedlicher Definitionen und fehlender Standards werden heute mindestens vier verschiedene Cashflow-Größen unterschieden:

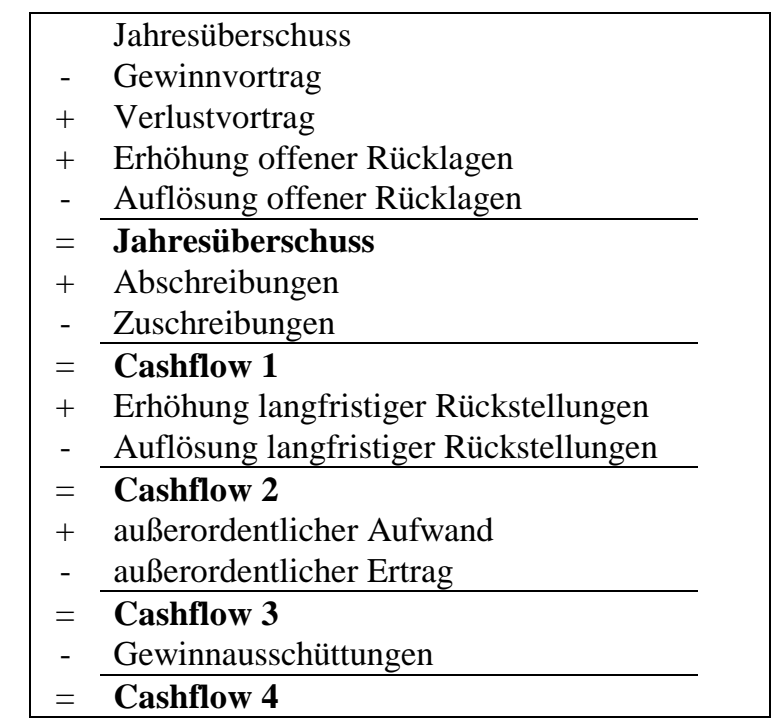

Tab. 39: Allgemeine Berechnung des Cashflow

\footnotetext{
179 Vgl. Küting / Weber (Bilanzanalyse, 1993) Seite $122 \mathrm{f}$.

180 Wöhe (Bilanzierung, 1997) Seite 284.

181 Wöhe (Bilanzierung, 1997) Seite 284.
} 
Eine weitere Möglichkeit besteht darin, die Gewinn- und Verlustrechnung als Ausgangspunkt der Cashflow-Berechnung heranzuziehen. $\frac{182}{}$ Aufbauend auf dem Bilanzgewinn wird im Planspiel nur der Cashflow 2 berechnet. Dies reicht aus, um allen Teilnehmern und auch der Spielleitung eine vergleichbare Größe der Finanzierungskraft anzugeben.

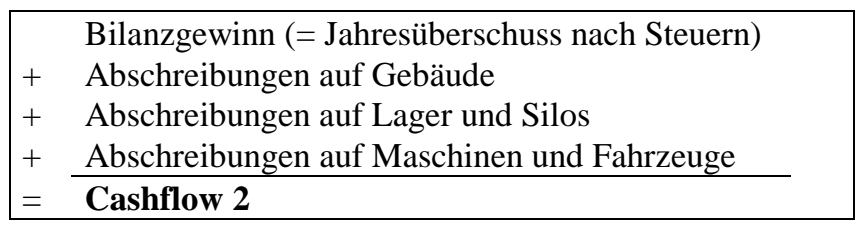

Tab. 40: Berechnung des Cashflow bei LogisticPLUS

Eine weitere Kennzahl ist die Eigenkapitalrentabilität. Diese ist eng mit dem Begriff des Leverage-Effekts verknüpft. Vereinfacht sagt dieser aus, das eine Erhöhung des Fremdkapitalanteils eine Erhöhung der Rentabilität des Eigenkapitals und damit des Marktwertes einer Unternehmung bewirkt („Hebel-Effekt“). ${ }^{1.83}$

\begin{tabular}{|l|r|c|c|}
\cline { 2 - 4 } \multicolumn{1}{c|}{} & Fall 1 & Fall 2 & Fall 3 \\
\hline Gesamtkapital & $100,-$ & $100,-$ & $100,-$ \\
\cline { 2 - 4 } Fremdkapital & $0,-$ & $25,-$ & $75,-$ \\
Eigenkapital & $100,-$ & $75,-$ & $25,-$ \\
\hline Gewinn vor Fremdkapitalzinsen & $10,-$ & $10,-$ & $10,-$ \\
Fremdkapitalzinsen 7 \% & $0,-$ & 1,75 & 5,25 \\
\cline { 2 - 4 } Gewinn & $10,-$ & 8,25 & 4,75 \\
\hline Eigenkapitalrentabilität & $10 \%$ & $11 \%$ & $19 \%$ \\
\hline
\end{tabular}

Tab. 41: Beispiel Eigenkapitalrentabilität

Im Planspiel gilt dabei folgende Definition: Eigenkapitalrentabilität $=\frac{\text { Gewinn }}{\text { Eigenkapital }}$. Das Eigenkapital besteht dabei aus dem gezeichneten Kapital und einem eventuellen Gewinn- oder Verlustvortrag aus den vergangenen Perioden. Dabei wird der Erfolg der aktuellen Periode nicht berücksichtigt.

182 Wöhe (Bilanzierung, 1997) Seite 286.

183 Vgl. dazu Bea et. al (ABWL, 2000) Seite 334, Schierenbeck (BWL, 2000) Seite 358 ff., Wöhe (Bilanzierung, 1997) Seite 265 oder Wöhe / Bilstein (Unternehmensfinanzierung, 1988) Seite $312 \mathrm{f}$. 


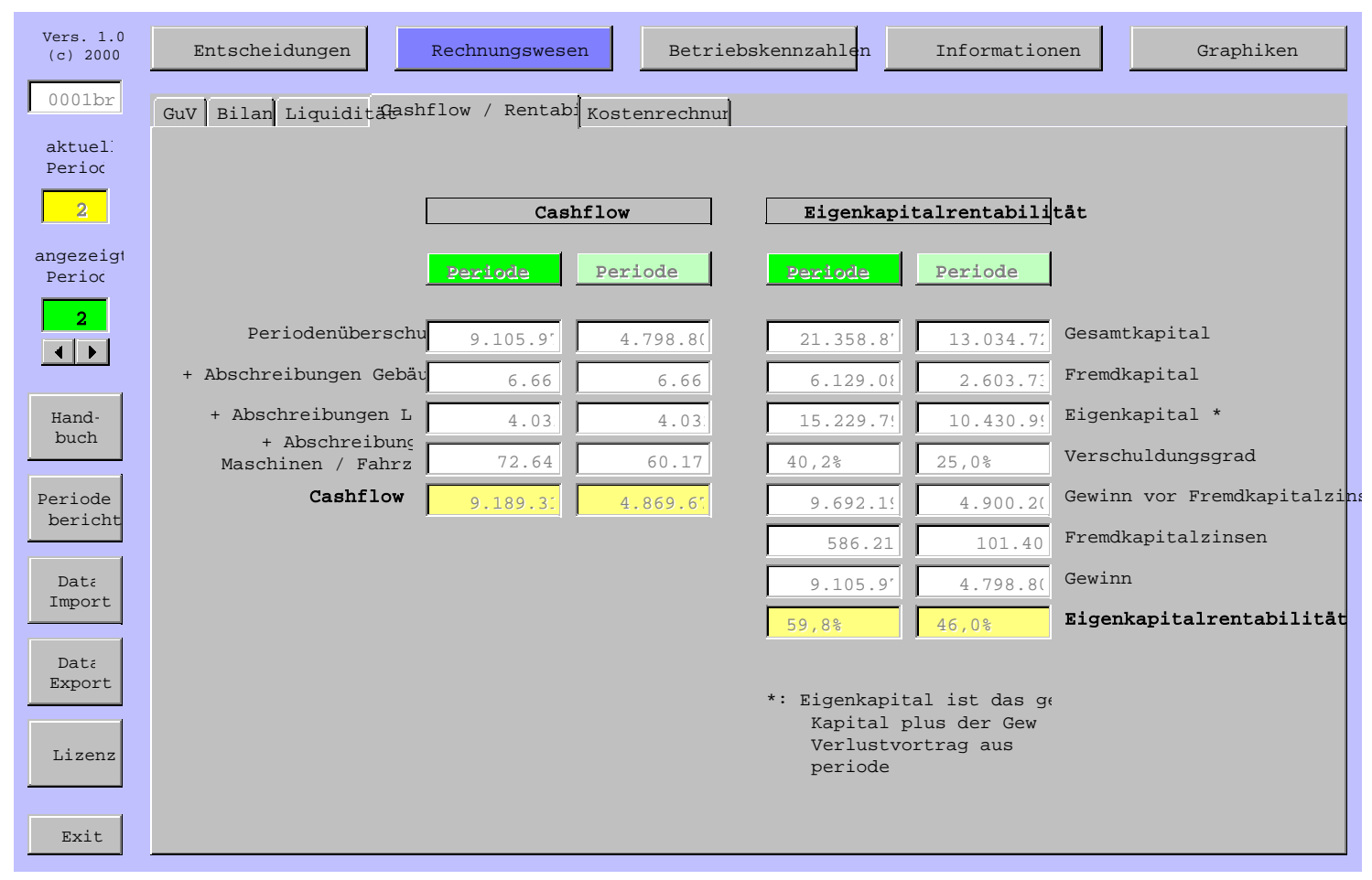

Abb. 45: Ausgabemaske Cashflow und Eigenkapitalrentabilität

In dieser Maske werden die beiden Größen Cashflow und Eigenkapitalrentabilität gemäß der getroffenen Definition dargestellt. Dabei wird bei der Eigenkapitalrentabilität als weitere finanzwirtschaftliche Größe auch der Verschuldungsgrad angegeben: Verschuldungsgrad $=\frac{\text { Fremdkapital }}{\text { Eigenkapital }}$. Die sogenannte vertikal Kapitalstrukturregel sagt aus, dass das Verhältnis Eigenkapital zu Fremdkapital eine bestimmte Mindestrate nicht übersteigen sollte. Am häufigsten wird dabei ein Verhältnis von Eigen- zu Fremdkapital von $1: 1$ gefordert. 184

\subsection{Einschlägige Betriebskennzahlen}

Im Menü Betriebskennzahlen werden ausnahmslos Informationen aus dem eigenen Unternehmen zur Verfügung gestellt. Immer wenn Entscheidungen innerhalb des Unternehmens verändert werden (Planung), erfolgt eine Neuberechnung zahlreicher Kennzahlen.

184 Wöhe (Bilanzierung, 1997) Seite 262. 
Weitere (externe) Informationen wie z.B. Marktvolumen oder veröffentlichte Zahlen der Konkurrenten werden im Menü Marktinformationen beschrieben.

\subsubsection{Vertrieb}

Die Vertriebskennzahlen beschreiben

1. die effektiven Aufwendungen für Werbung im In- und Ausland,

2. den fakturierten Umsatz (das ist der den Kunden in Rechnung gestellte Wert) sowie

3. den Absatz und die erzielten Preise der beiden Produkte Singulus und Combilus in Bezug auf die Märkte Inland, Export (Ausland) und gegebenenfalls Bei Ausschreibungen.

$\mathrm{Zu}$ beachten ist, dass der hier ausgewiesene Umsatz nicht vollständig zu Einzahlungen führt. Aufgrund des Zahlungsverhalten der Kunden werden z.B. nur 80 Prozent der fakturierten Umsätze noch in der Periode gebucht. Dies wirkt sich bei der Liquiditätsrechnung aus.

Weitere Kennzahlen wie Exportanteil der ausgelieferten Produkte sowie anteiliger Produktumsatz vom Gesamtumsatz runden dieses Menü ab. 


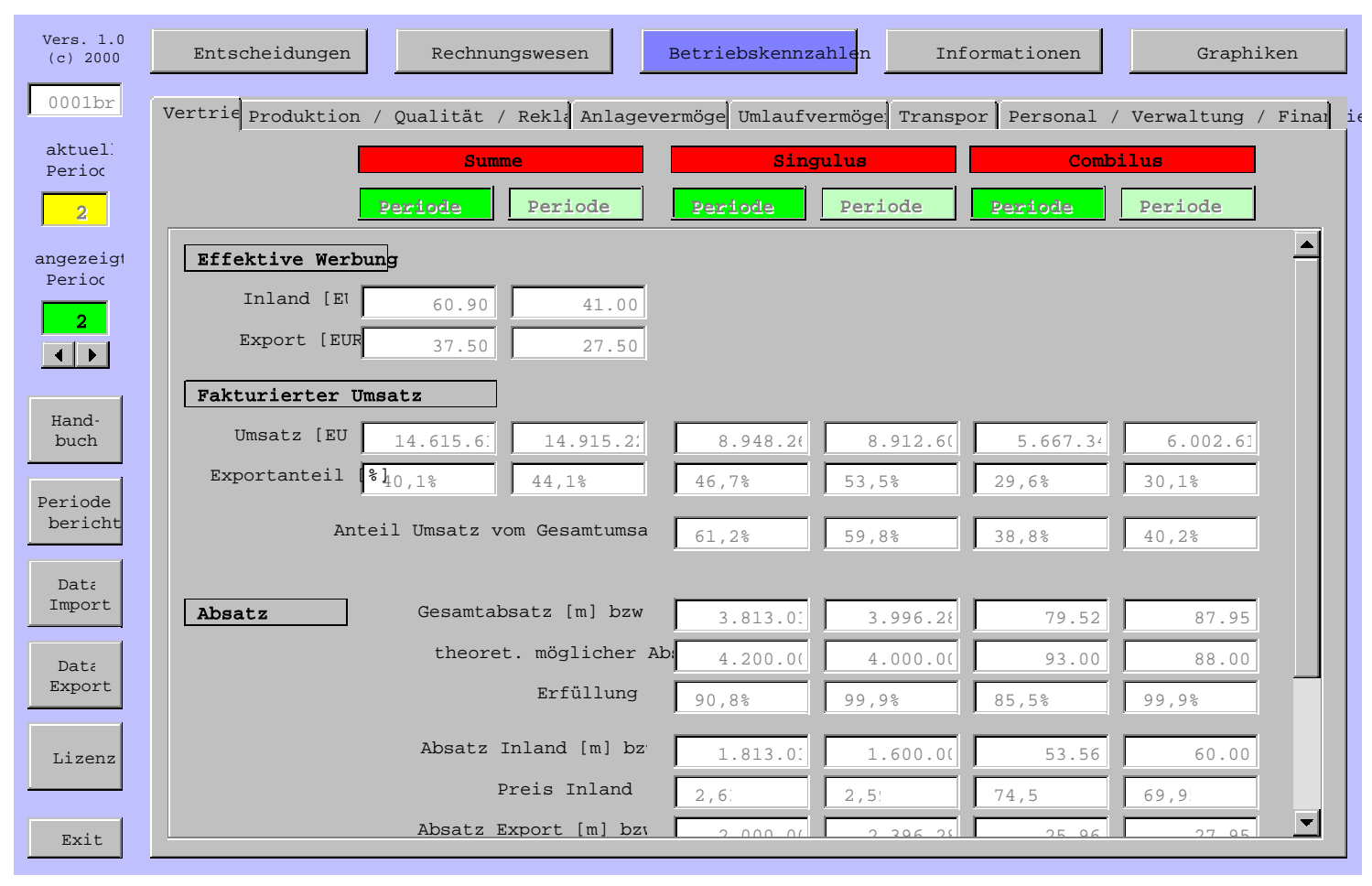

Abb. 46: Ausgabemaske Kennzahlen Vertrieb

Auch hier sind einige weitere Absatzkennzahlen nicht auf der Bildschirmmaske erkennbar. Im Computerprogramm kann mit der rechten Bildlaufleiste in den unteren Bereich gescrollt werden. In diesem Fall fehlen die Preise Absatz Export sowie die kompletten Zahlen (Preis und Menge) der eventuell gewonnenen Ausschreibungen. 
In der im Verzeichnis GRAPHICS liegenden Datei werden folgende Graphiken erzeugt:

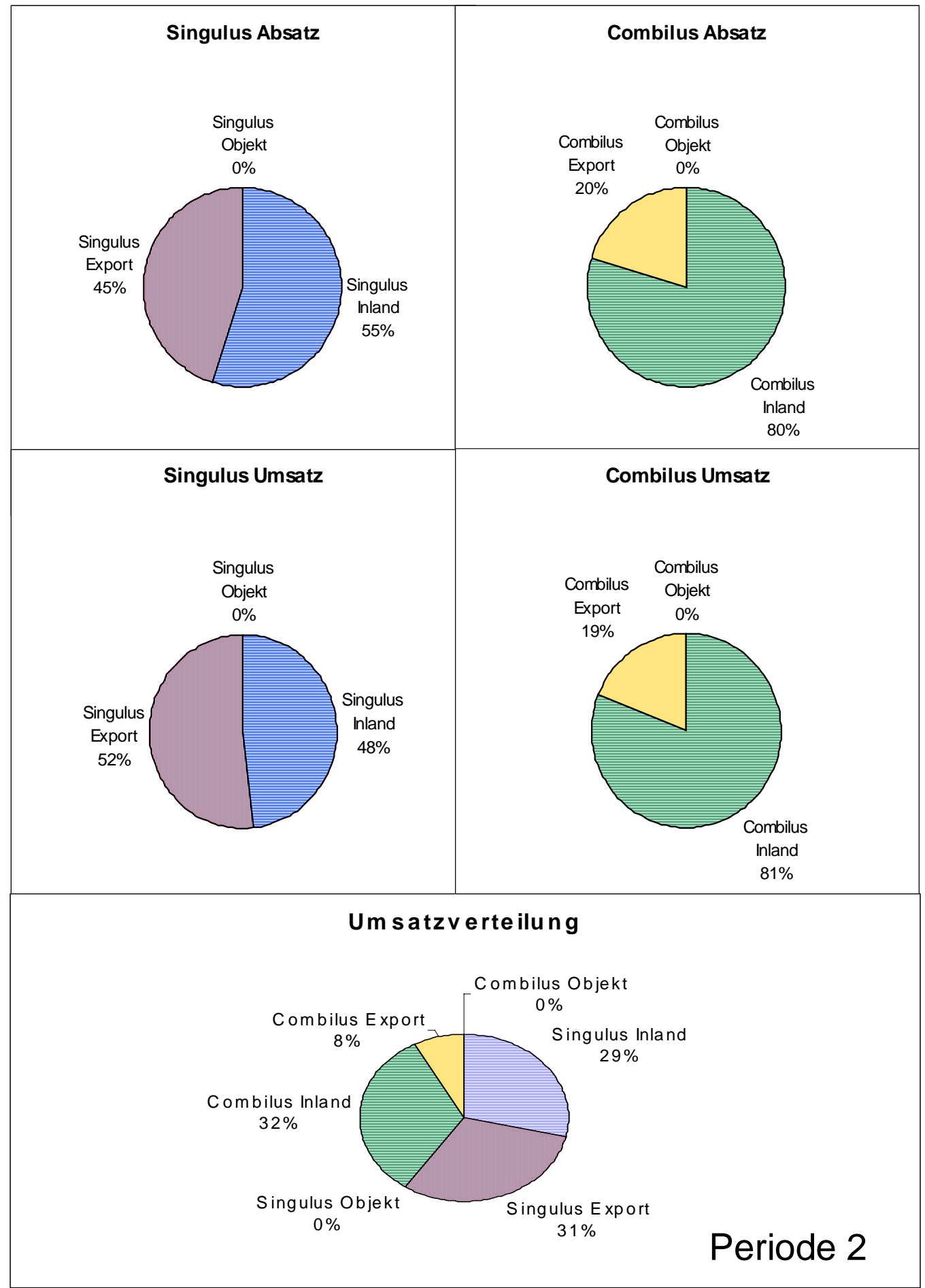

Abb. 47: Ausgewählte Vertriebskennzahlen

Sehr deutlich erkennbar ist die Aufteilung des Absatzes auf die beiden Produkte sowie die Aufteilung des Umsatzes auf die beiden Produkte in den relevanten Märkten. In obigem Beispiel erkennt man z.B. die Stärke von Combilus im Inland sowie die 
Preisstärke von Singulus im Ausland. So machen 45 Prozent des Absatzes von Singulus 52 Prozent des Umsatzes mit Singulus aus.

\subsubsection{Produktion, Qualität und Reklamationen}

Im Menübereich Produktion werden die definitiv produzierten Produkte 1 und 2 sowie die definitiv produzierten Verkaufsartikel Singulus und Combilus ausgewiesen. Dabei wird auch der bei der Montage erkannte Ausschuss in Stück und in Prozent der produzierten Menge dargestellt. In das Verkaufsartikel-Lager kommen nur fehlerlos produzierte Artikel, das Ausschuss-Lager nimmt den Rest auf.

Weiterhin werden die für den Produktionsausschuss relevanten effektiven Investitionen wie QM-System, Personalweiterbildung, Aufwand für Wartung und für F\&E aufgezeigt. Hieraus ergeben sich Rückschlüsse auf gegebenenfalls nötige Veränderungen bei den Investitionen.

Man erhält zudem einen Überblick über die in dieser Periode angefallenen Reklamationen. Diese werden jedoch erst in drei Perioden in Form einer Rechnungsstellung wirksam. 


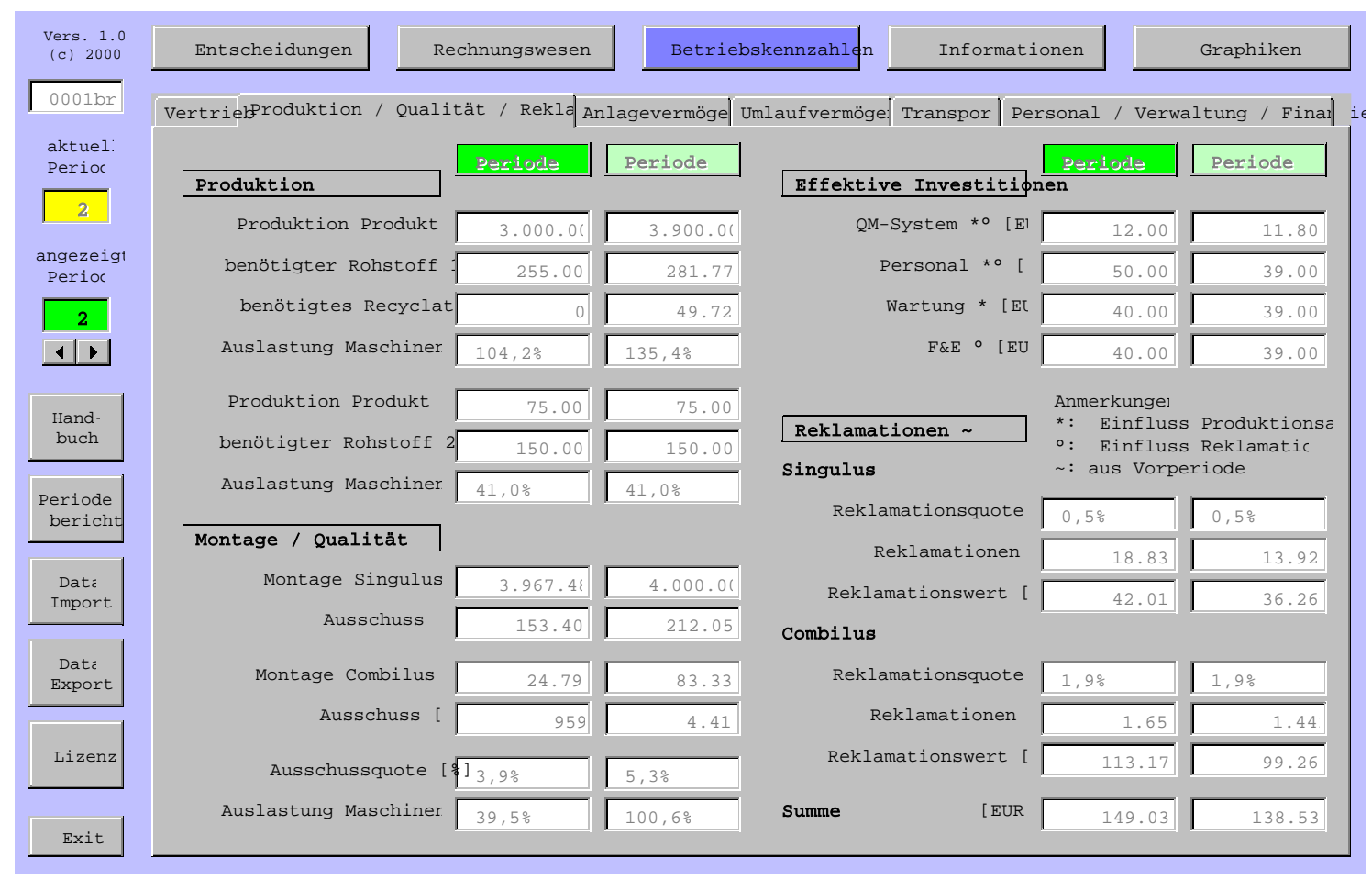

Abb. 48: Ausgabemaske Kennzahlen Produktion, Qualität und Reklamationen

Die obige Maske gibt u.a. einen sehr guten Überblick über die Qualität der Produkte. So wird unter „Montage / Qualität“ neben der absoluten Menge bzw. Anzahl der hergestellten bzw. montierten Produkte der Ausschuss in m, Stück bzw. Prozent dargestellt. Weiterhin erfährt man, wie viele Reklamationen eingegangen sind und welche Kosten dahinter stehen. Dies prozentualen Kennzahlen beziehen sich dabei auf die Vorperiode.

\subsubsection{Anlagevermögen}

Das Anlagevermögen besteht grundsätzlich aus Grundstücken, Gebäuden, Silos, Maschinen und Fahrzeugen. Es gelten folgende Abschreibungsrichtlinien:

1. Die betriebseigenen Grundstücke werden im Planspiel nicht gesondert betrachtet. Gemäß der einschlägigen Gesetze werden die Grundstücke mit den Anschaffungskosten bewertet. 
2. Gebäude sind Verwaltungsgebäude oder Lager. Beide werden periodisch nach den steuerrechtlichen Grundsätzen abgeschrieben.

3. Silos werden über 10 Jahre ( $=60$ Perioden) abgeschrieben.

4. Maschinen werden ebenfalls über 10 Jahre abgeschrieben.

5. Gabelstapler werden über 5 Jahre (= 30 Perioden) abgeschrieben.

Für Lager, Silos, Maschinen und Gabelstapler gilt gleichermaßen, dass diese nach Ablauf der Abschreibung nicht mehr benutzt werden können.

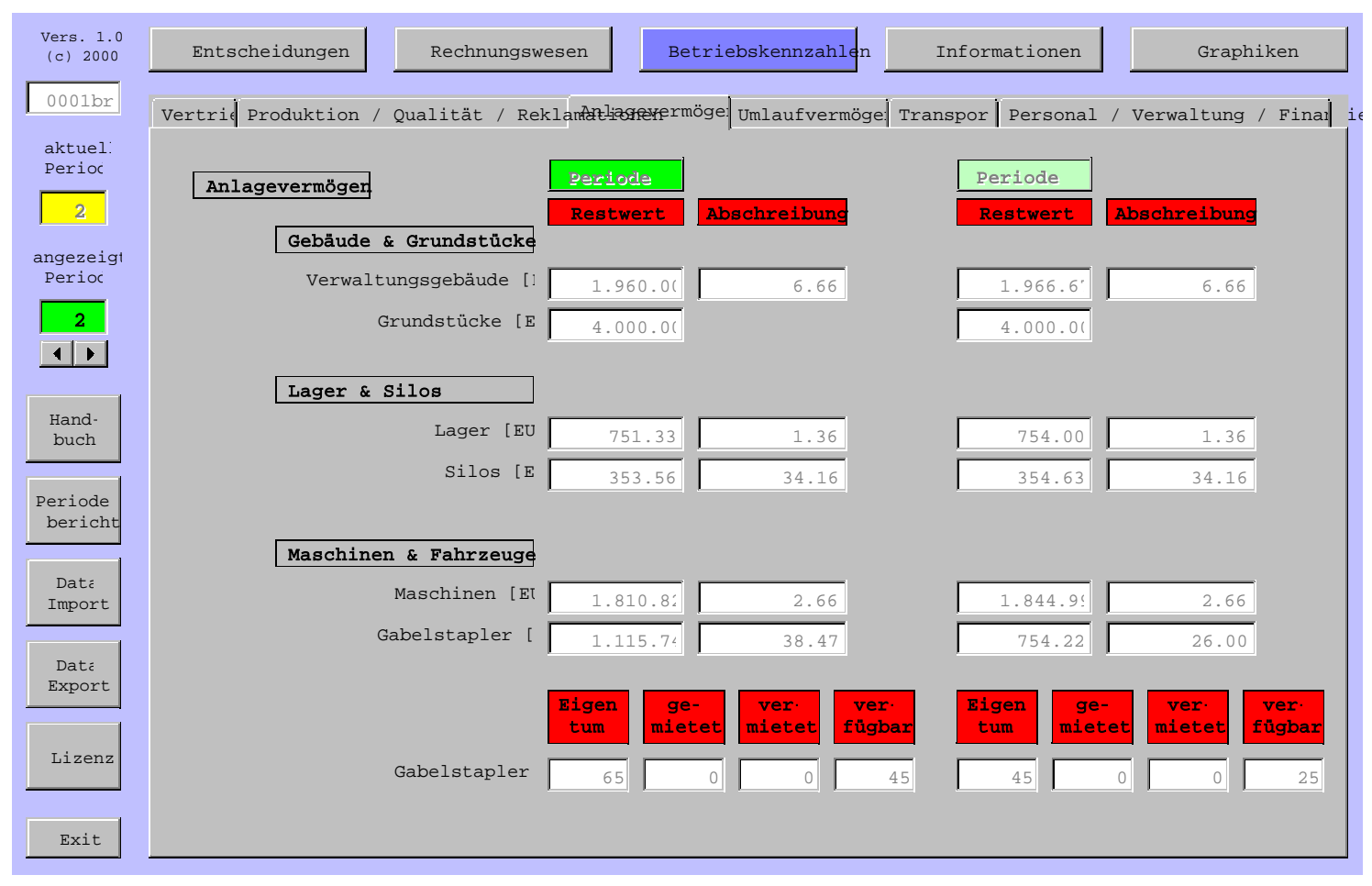

Abb. 49: Ausgabemaske Kennzahlen Anlagevermögen

Das Anlagevermögen wird in Gebäude \& Grundstücke, Lager \& Silos und in Maschinen \& Fahrzeuge unterschieden. Die Grundstücke werden nicht, das restliche Anlagevermögen nach den rechtlichen Bestimmungen abgeschrieben. Im unteren Bereich wird die Anzahl der Gabelstapler angezeigt. Hierbei werden insbesondere die verfügbaren Gabelstapler angezeigt. Die hier erkennbaren Unterschiede resultieren aus der Regel, dass bei der Beschaffung eine Periode durch die Inbetriebnahme „überbrückt”“ werden muss. 


\subsubsection{Umlaufvermögen}

Im Planspiel existieren insgesamt sechs Lagerstellen: Silo für Rohstoff 1, Silo für Rohstoff 2, Halbfabrikat-Lager, Fertigfabrikat-Lager, Ausschuss-Lager und Silo für Recyclat. Diese können gegebenenfalls aus mehreren unterschiedlichen physischen Einheiten bestehen. $\frac{185}{}$ Dabei wird jedoch für die finanzielle und Bestandsbewertung das jeweilige Gesamtlager betrachtet.

Für jede dieser Lagerstellen werden im Planspiel

1. die im Lager liegenden Gesamt- und Stückwerte der Rohstoffe, Produkte, Verkaufsartikel und des Recyclats,

2. die im Lager liegenden Mengen der Rohstoffe, Produkte, Verkaufsartikel, Recyclat und Ausschuss,

3. das jeweils benötigte Volumen,

4. die Summe der bereitgestellten Lagervolumen (Lagerkapazität),

5. die Auslastung der jeweiligen Lagerstellen sowie gegebenenfalls

6. die Reichweite der Produkte in Arbeitstagen $\underline{186}$ angegeben.

Das Recyclat und den Ausschuss bewertet man unabhängig ihrer Herkunft. Beim Recyclat nimmt man bei der Volumenberechnung das Gewicht des Rohstoffes 1 an.

\footnotetext{
185 So kann z.B. das Rohstofflager 1 aus verschiedenen Silos bestehen.

186 Aufgrund der Festlegung, dass eine Periode über 2 Monate geht, wird angenommen, dass in einer Periode regulär 40 Tage gearbeitet wird.
} 


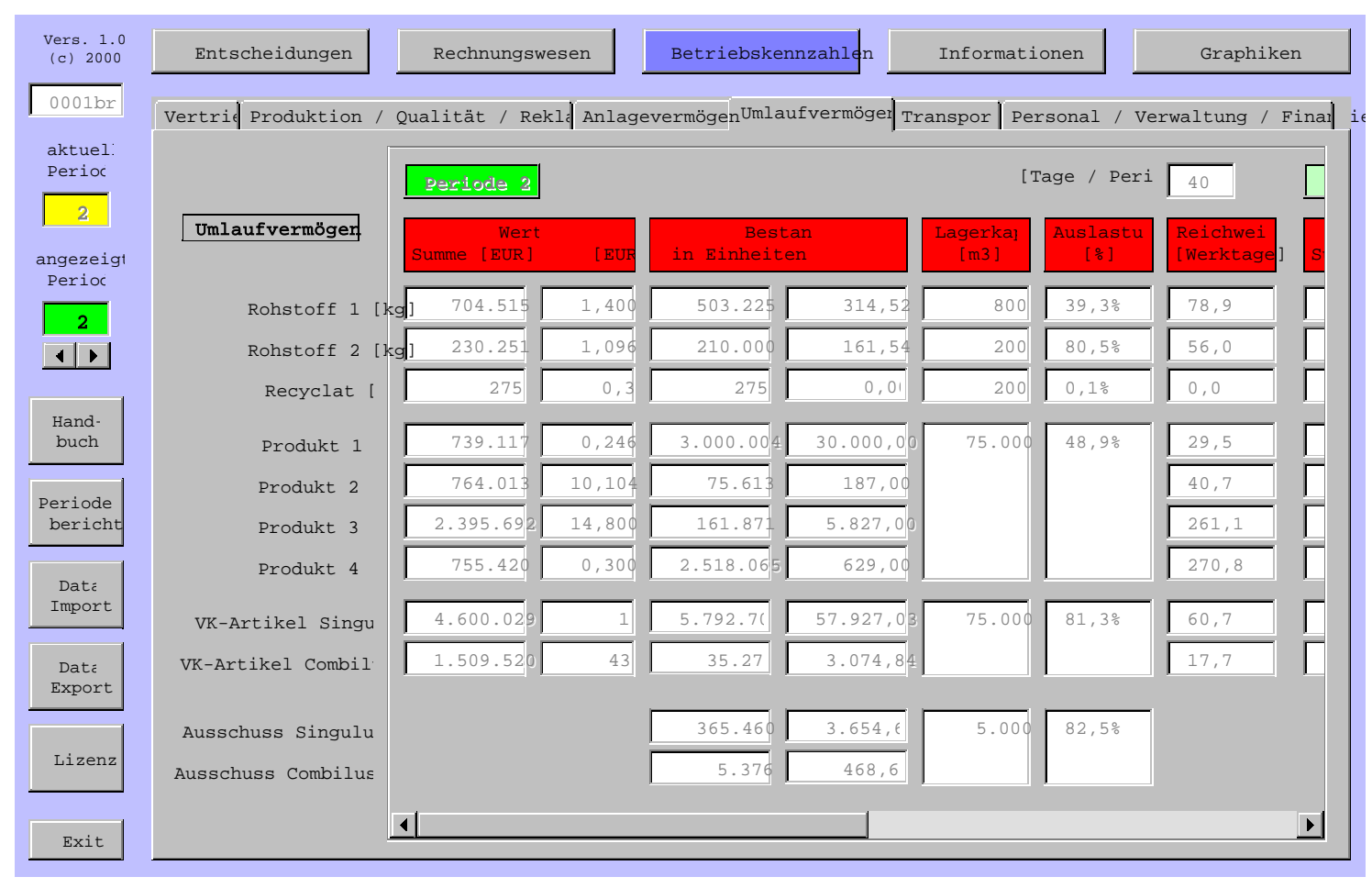

Abb. 50: Ausgabemaske Kennzahlen Umlaufvermögen

Auf dieser Maske ist nur die aktuelle geplante Periode auf den ersten Blick erkennbar (Periode 2). Die vergangene Periode mit Ist-Werten steht rechts und kann mit der unteren Laufleiste sichtbar gemacht werden.

Gemäß der Festelegung der Produktionsstruktur in Kapitel 4.2 - Leistungsströme ist das Halbfabrikatlager als ein Lagerhaus für alle vier Produkte anzusehen. Dies wird in obiger Maske durch die beiden großen weißen Felder dargestellt. Derzeit stehen für Halbfabrikate $75.000 \mathrm{~m}^{3}$ Lagervolumen zur Verfügung, dies ist zu 48,9 Prozent ausgelastet. 
In der Datei GRAPHICS findet sich die nachfolgende Darstellung zur Lagerauslastung.

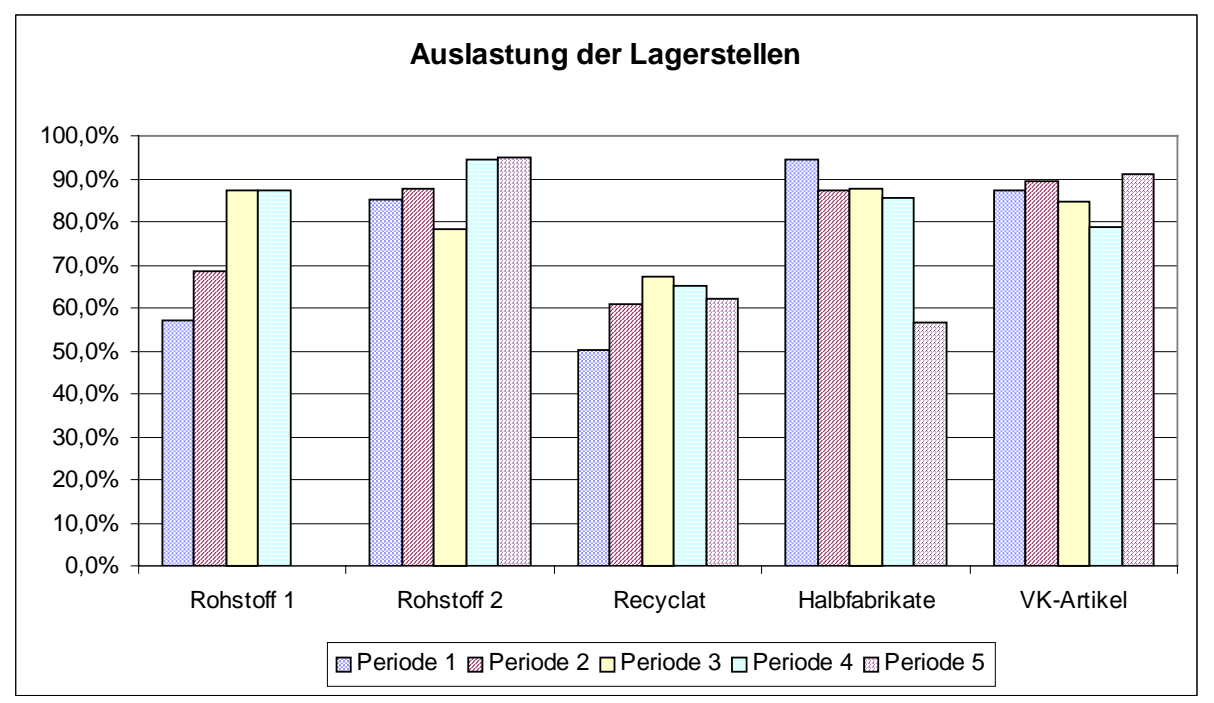

Abb. 51: Graphische Darstellung Auslastung der Lagerstellen

In diesem Bild werden die Zustände und damit die Veränderungen über die letzten fünf Perioden dargestellt. Somit besteht die Möglichkeit der genauen Analyse der Entwicklung der Lagerstellen. In obigem Beispiel (aktuelle Periode 5) nähern sich bis auf das Halbfabrikatlager die Auslastungen der Lagerstellen in Richtung Vollauslastung. 


\subsubsection{Transporte}

Die Transportkennzahlen schlüsseln die sieben unterschiedlichen innerbetrieblichen Transporte nach $\mathrm{m}^{3}$ und nach benötigten Gabelstaplerkapazitäten auf. Sollten die bereitgestellten Gabelstapler nicht ausreichen, werden die Güter zu höheren Kosten trotzdem transportiert.

\begin{tabular}{|l|l|l|}
\hline & Tätigkeit & Ziel \\
\hline 1 & Transport Produkt 1 und 2 & Lager Halbfabrikate \\
\hline 2 & Abladung Produkt 3 und 4 & Lager Halbfabrikate \\
\hline 3 & Transport Produkt 1 bis 4 & Montage \\
\hline 4 & Transport VK-Artikel & Lager Artikel \\
\hline 5 & Transport Ausschuss & Ausschuss-Lager \\
\hline 6 & Abfrachtung Artikel & LKW \\
\hline 7 & Transport Recyclat & Recycling \\
\hline
\end{tabular}

Tab. 42: Innerbetriebliche Transporte

Beim Versandtransport der VK-Artikel Singulus und Combilus wird detailliert nach Märkten und Produkten unterschieden. Dabei werden einerseits transportierte Mengen, andererseits angefallene Kosten aufgezeigt.

Hier haben die Spielteams bereits in der Planungsphase die Möglichkeit, eventuell zu geringe Bestellmengen von LKW oder Gabelstapler zu erkennen: Die Auslastung steigt über 100 Prozent. 


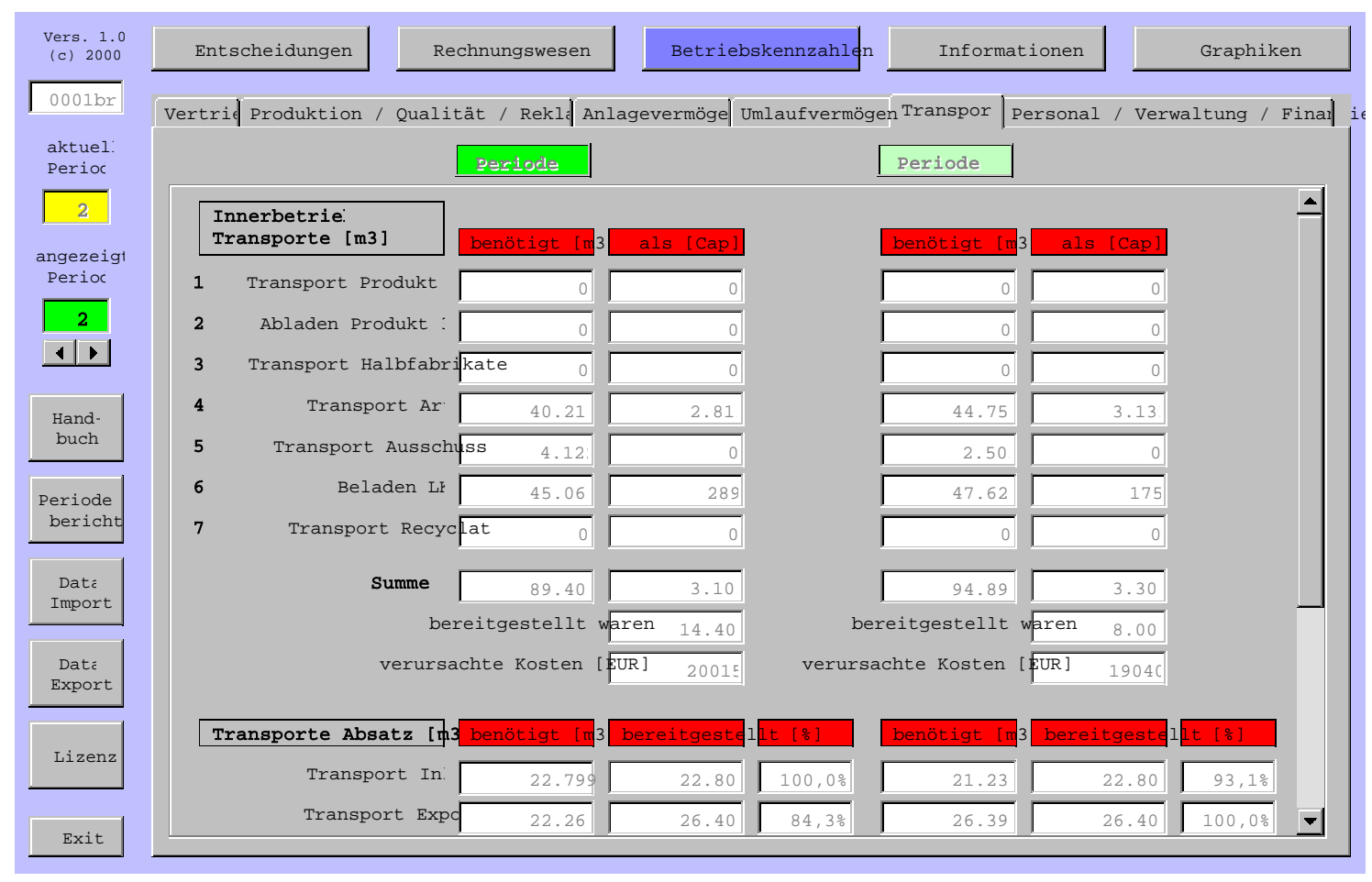

Abb. 52: Ausgabemaske Kennzahlen Transporte

Weitere Kennzahlen zum Transport Absatz erhält man mit der rechten Scroll-Leiste. Es sind dies Transportvolumen Objekt, Summe der Transporte, Transportkosten Inland, Export, Objekt sowie die Summe der Transportkosten.

\subsubsection{Personal, Verwaltung und Finanzierung}

Dieser Bereich listet Fach- und Hilfsarbeiter nach Sollbestand und einsetzbaren Arbeitern auf. Weiterhin wird die Auslastung angegeben.

Beträgt die Auslastung der Facharbeiter über 100 Prozent, sind die geplanten Fertigungs- und Montageaktivitäten mit dem Mitarbeiterstamm nicht durchführbar. Es werden teurere Leiharbeiter bzw. Überstunden benötigt. Dasselbe gilt für Facharbeiter und die innerbetrieblichen Transporte.

Überdies erhält man Auskunft über gezahlte Löhne und die geleisteten Sozialabgaben. Gehälter und Sozialabgaben des Vorstandes werden hier mit eingerechnet. Die Höhe der 
Sozialabgaben ist im Menü Entscheidungen wählbar und hat Einfluss auf den Krankenstand der Mitarbeiter (Krankheitsquote).

Das nachfolgende Bild zeigt das Verhältnis der Fach- zu Leiharbeitern. Hierbei wird unterschiedne in die absolute Anzahl sowie in die Anzahl der einsetzbaren Arbeiter.

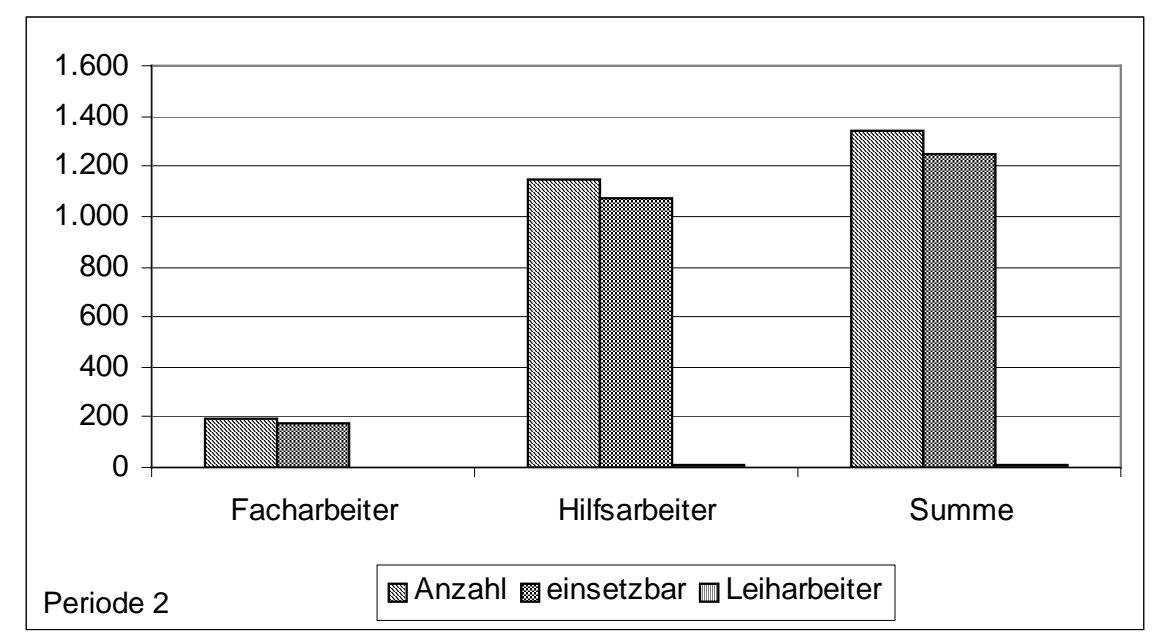

Abb. 53: Graphische Darstellung Personalstand

Weiterhin erhält man Informationen über die angefallenen Kosten der Verwaltung.

Dabei wird in umsatzabhängige und in mitarbeiterabhängige Kennzahlen unterschieden. 


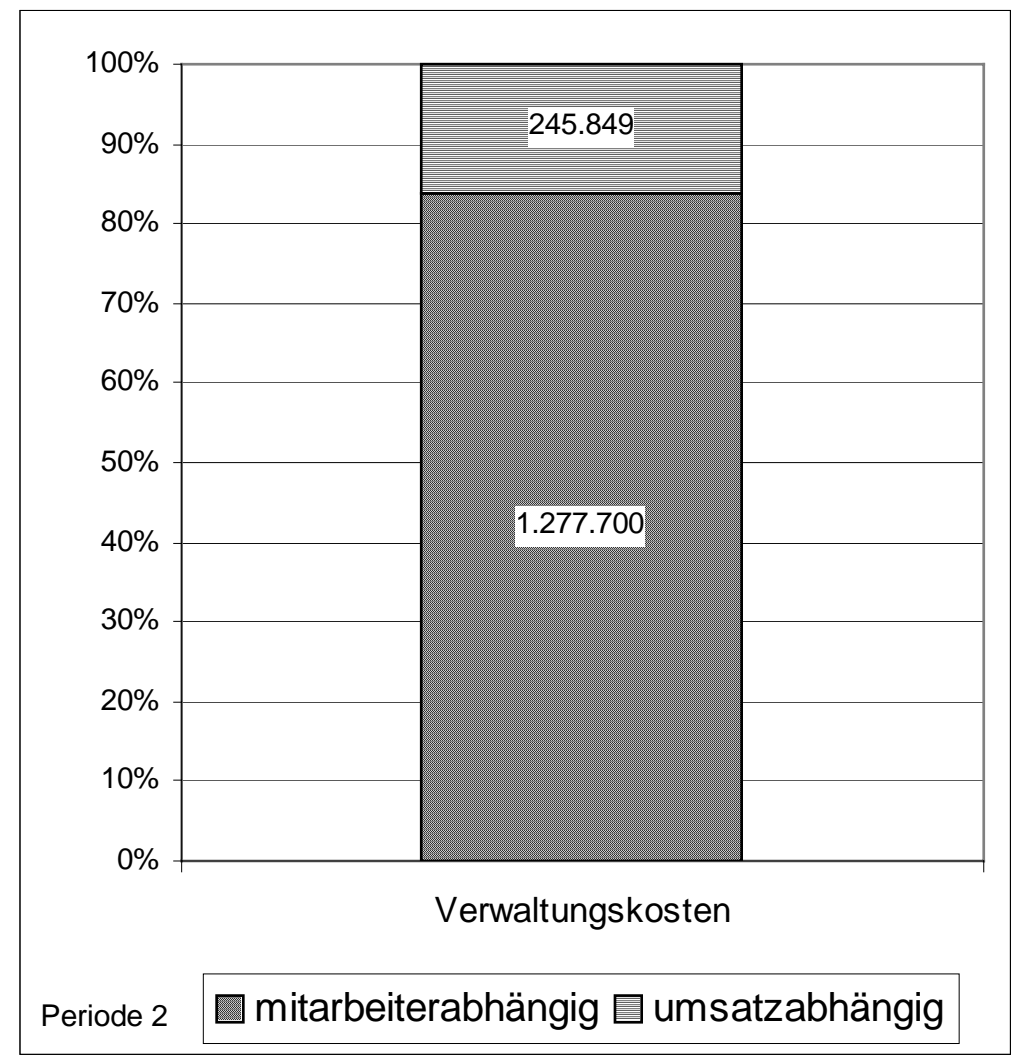

Abb. 54: Graphische Darstellung Kosten der Verwaltung

Im Bereich Finanzierung werden Informationen über das ausgeschöpfte Kreditvolumen, über in Anspruch genommenen Liquiditätskredit sowie über eigene Wertpapiere angezeigt. Durchschnittliche Zinssätze und angefallene Schuld- und Habenzinsen werden ebenfalls ausgewiesen. 


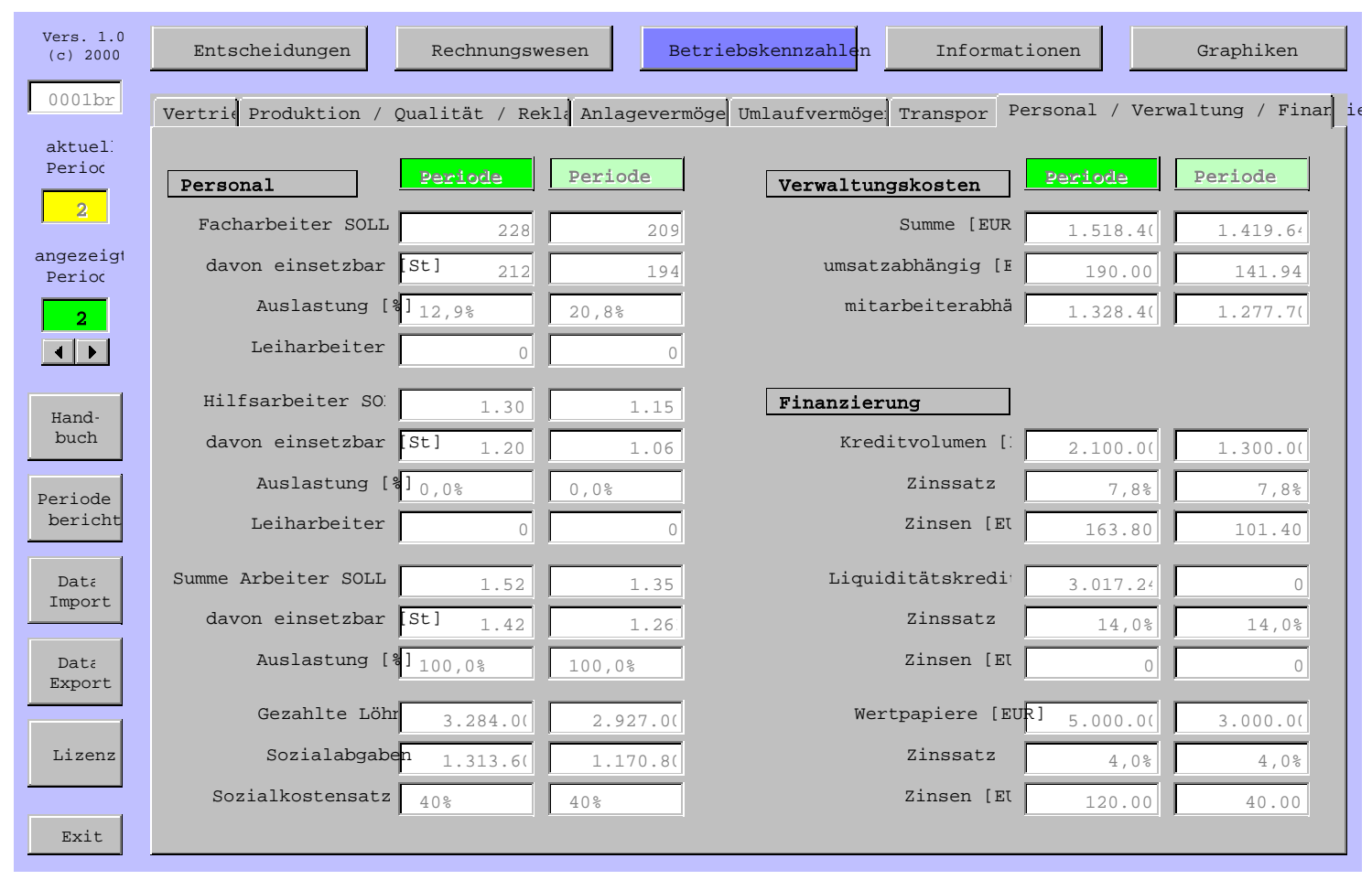

Abb. 55: Ausgabemaske Kennzahlen Personal, Verwaltung und Finanzierung

Die Auslastung beim Personal bedeutet, wie viele der einsetzbaren Kapazitäten durch die unterschiedlichen Tätigkeiten (z.B. Produktion, Montage oder Transporte) wirklich in Anspruch genommen wurden.

Die Verwaltungskosten werden nach umsatzabhängigen und mitarbeiterabhängigen Kosten aufgeschlüsselt.

Die Zinssätze der Finanzierung sind p.a. Zinssätze, die resultierenden Zinsen werden entsprechend der festgelegten Periodendauer von 2 Monaten auf ein Sechstel reduziert. 


\subsection{Marktinformationen}

\subsubsection{Allgemeines}

Das Planspiel stellt im Menüpunkt „Informationen“ verschiedene wichtige Zahlen und Parameter zur Verfügung. Dabei wird unterschieden zwischen Marktpreisen und Parameter einerseits sowie Marktforschungsergebnisse andererseits.

\subsubsection{Informationen über Marktpreise}

Die Marktpreise / -infos können aktiv nur durch die Spielleitung beeinflusst werden. Dadurch hat sie die Möglichkeit, Einfluss auf die Erträge und Aufwendungen der Spieler zu nehmen. Die Preise und Informationen gelten für alle fünf Spieler gleichermaßen.

Im Bereich Einkauf werden Preise für Rohstoffe und Zukaufprodukte sowie die Gebühren für gegebenenfalls unnötige Anfahrten aufgezeigt. 


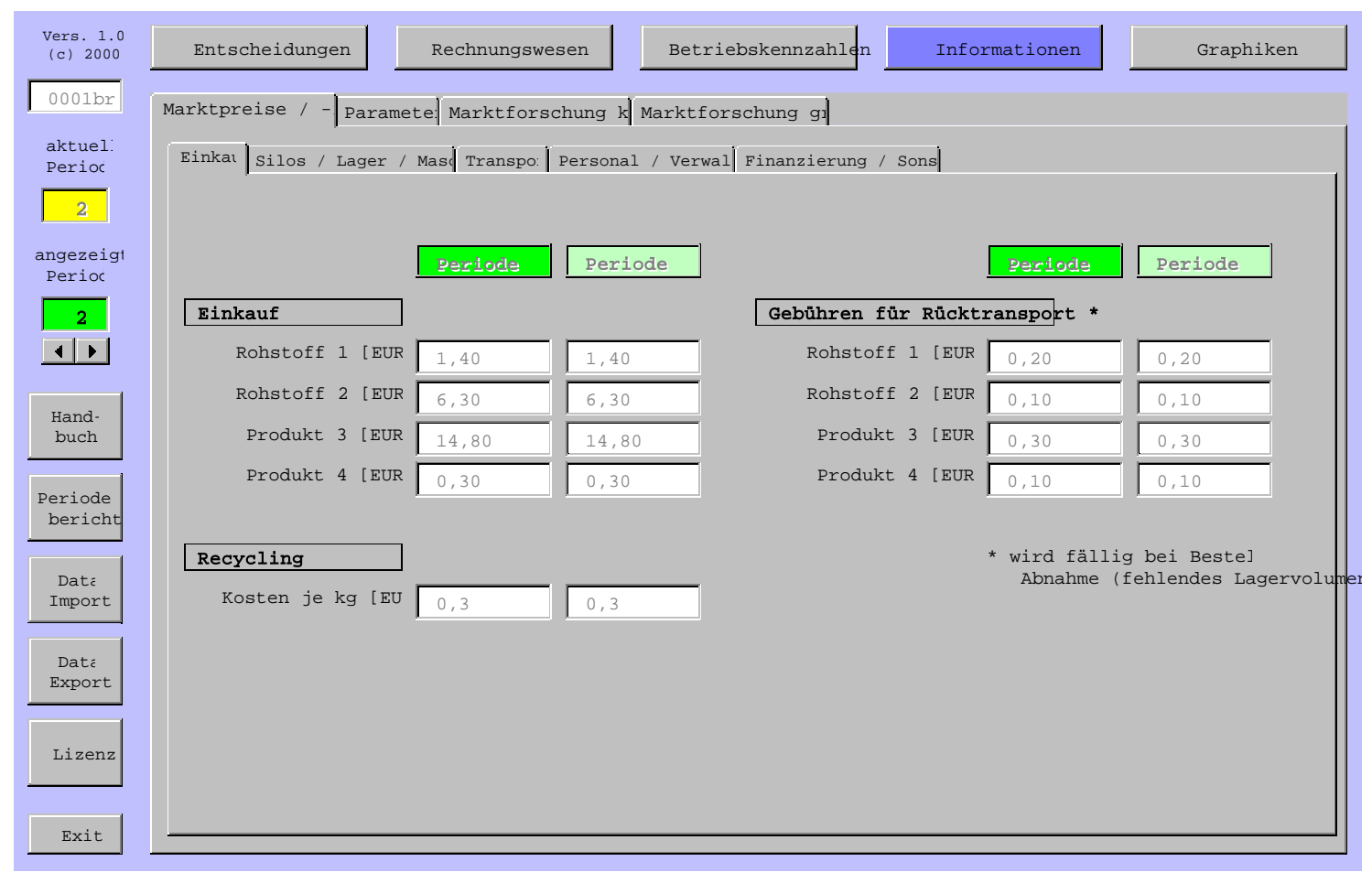

Abb. 56: Ausgabemaske Marktpreise Einkauf

Die Gebühren für unnötige Anfahrten werden fällig, falls bestellte Rohstoffe oder Zukaufprodukte aufgrund von Lagerengpässen nicht eingelagert werden können. Dies entspricht einem Rücktransport an den Lieferanten. 
Auf der Bildschirmmaske Silos / Lager / Maschinen erhält man Informationen über die Preise für Neuanschaffungen sowie erzielbare Einnahmen für Vermietungen.

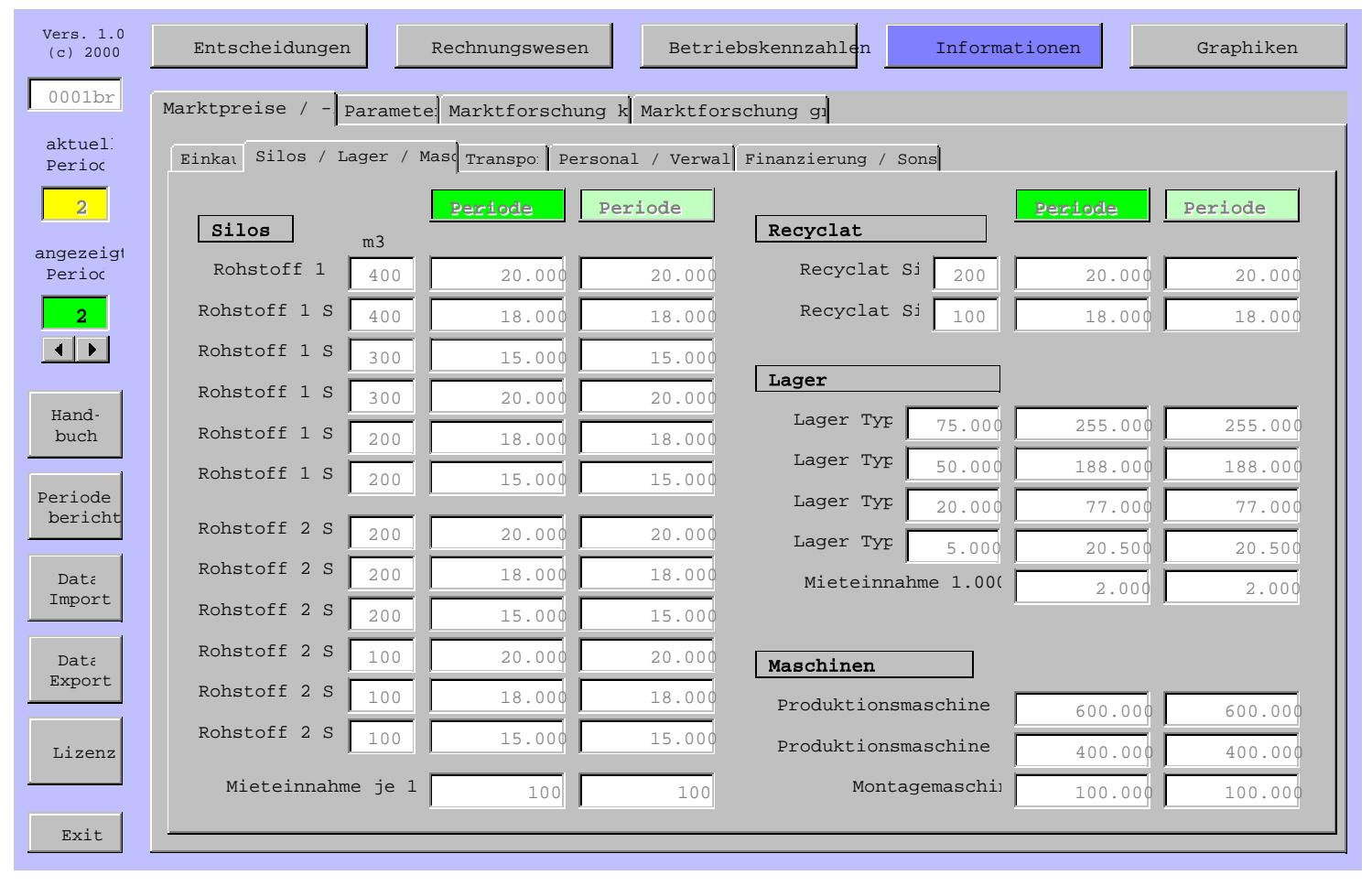

Abb. 57: Ausgabemaske Marktpreise Silos, Lager und Maschinen

Silos, Recyclat-Silos sowie Lager können in unterschiedlichen Größen aufgebaut werden. So gibt es z.B. Silos für 200, 300 und $400 \mathrm{~m}^{3}$ Inhalt. Lagergebäude stehen in den Größen 5.000, 20.000, 50.000 und $75.000 \mathrm{~m}^{3}$ zur Verfügung. Bei der Beschaffung ist zu berücksichtigen, dass erworbene Gebäude nicht wieder veräußert werden können, es ist lediglich eine Vermietung möglich. 
Unter Transporte werden die Kosten je transportiertem Kubikmeter und Markt angeben sowie die anfallenden Ausgaben für die Investition oder Anmietung von Gabelstaplern.

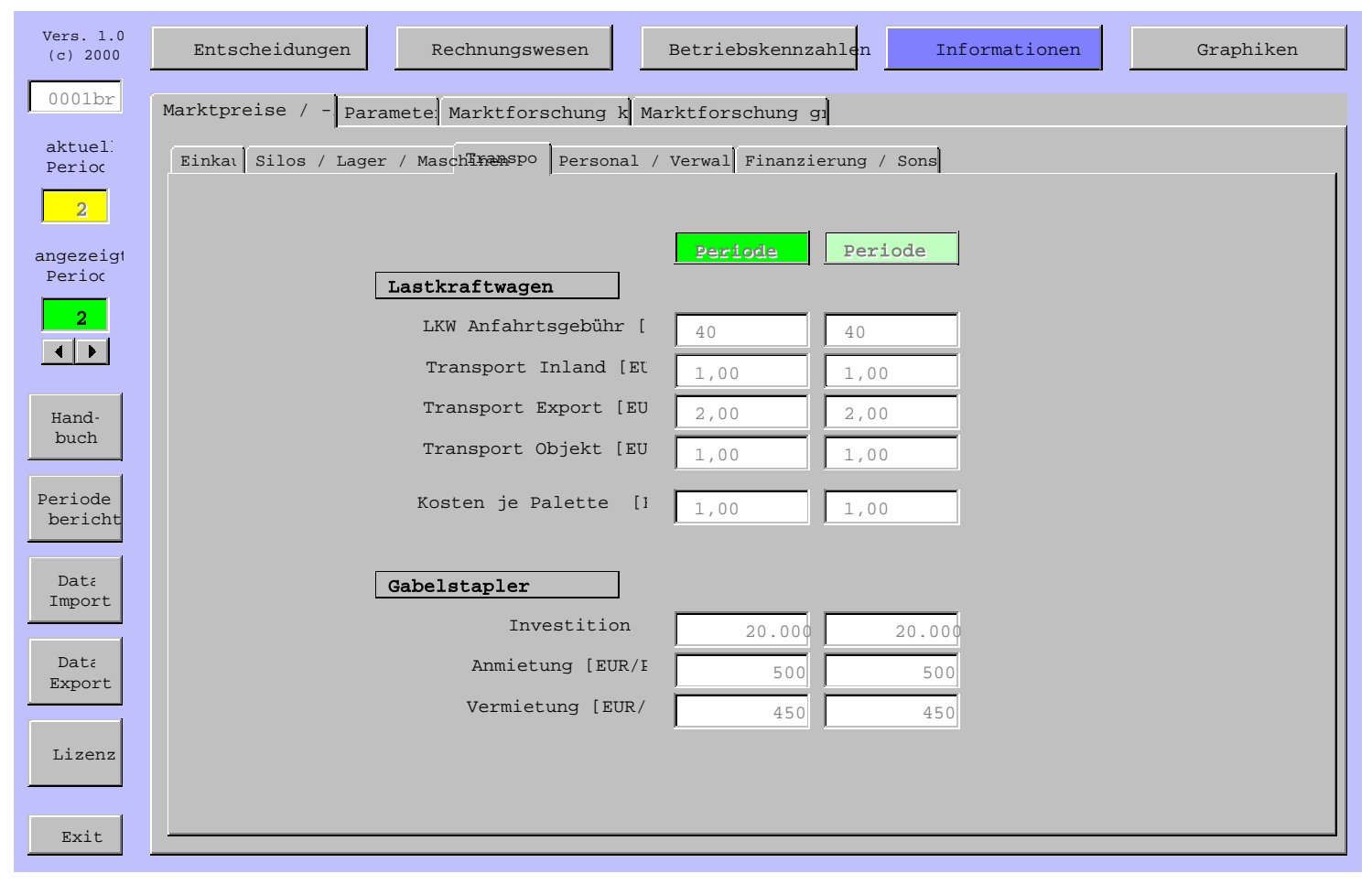

Abb. 58: Ausgabemaske Marktpreise Transport

Die Kosten für die Absatztransporte sind Fixkosten je bestelltem LKW sowie abhängige variable Kosten je transportiertem $\mathrm{m}^{3}$ für Inland, Export und Objektgeschäft. Für jedem transportierten $\mathrm{m}^{3}$ fällt weiterhin eine Palettengebühr an.

Die Gabelstapler können erworben oder je Periode angemietet werden. Sind zuviel Gabelstapler im eigenen Bestand können diese vermietet werden. Die Nachfrage hierfür ist unbegrenzt. 
Unter Personal / Verwaltung erhält man Informationen über Löhne, Einstellungs- und Entlassungsaufwendungen sowie über anfallende Mehrkosten beim Einsatz von Leihkräften.

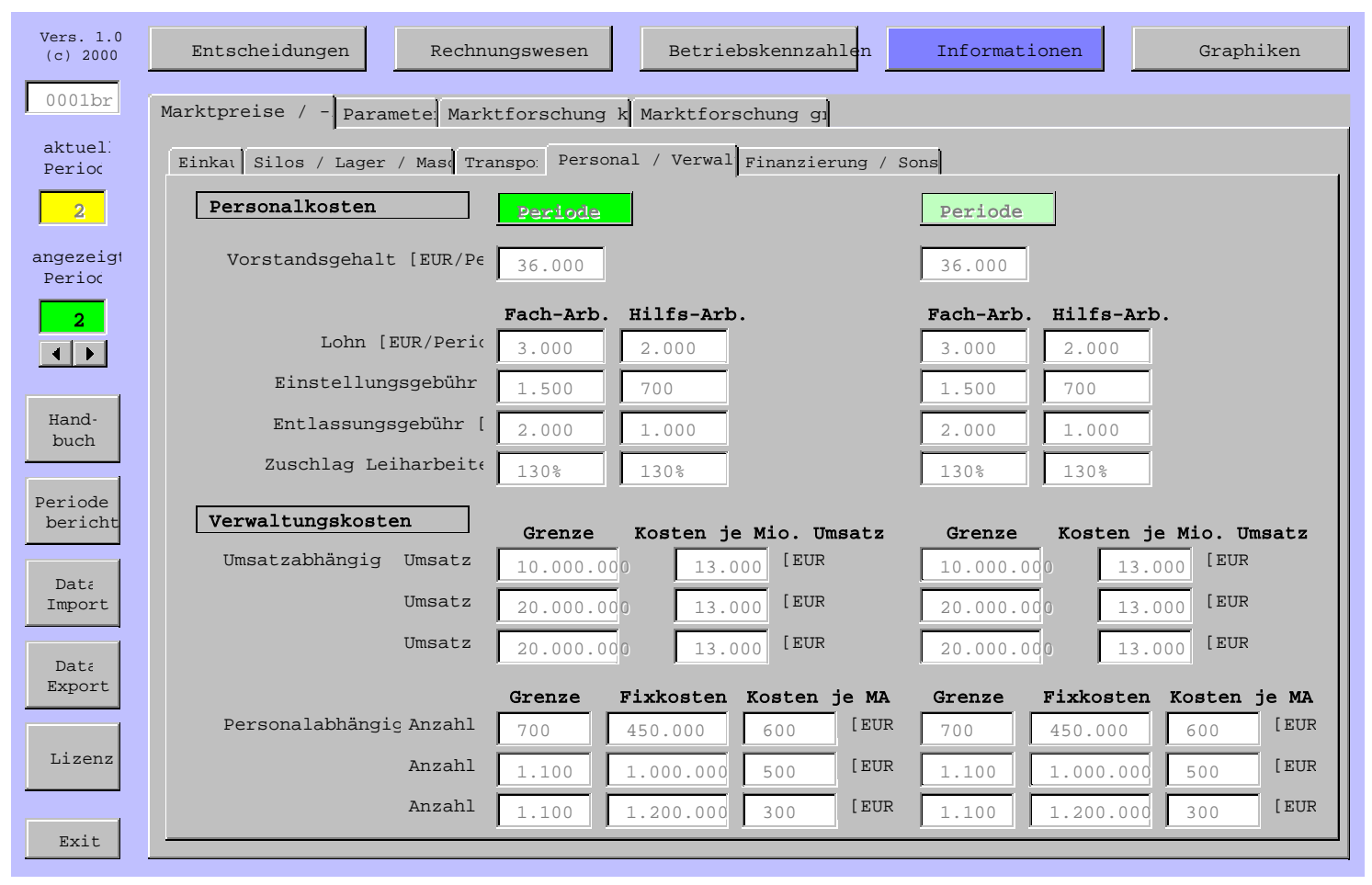

Abb. 59: Ausgabemaske Marktpreise Personal und Verwaltung

Weiterhin werden die Verwaltungskosten nach umsatzabhängigen und mitarbeiterabhängigen Kosten aufgeschlüsselt. Dabei sind fixe Kosten und variable Kosten zu addieren. So fallen bei einer Anzahl von 800 Mitarbeitern mitarbeiterabhängige Verwaltungskosten in Höhe von $1.000 .000+700 * 600+100 *$ $500=1.470 .000,-€$ an. 
Finanzen / Sonstiges gibt einen Überblick über die Geldmarktsituation sowie über gegebenenfalls anfallende Beratungsgebühren durch die Spielleitung.

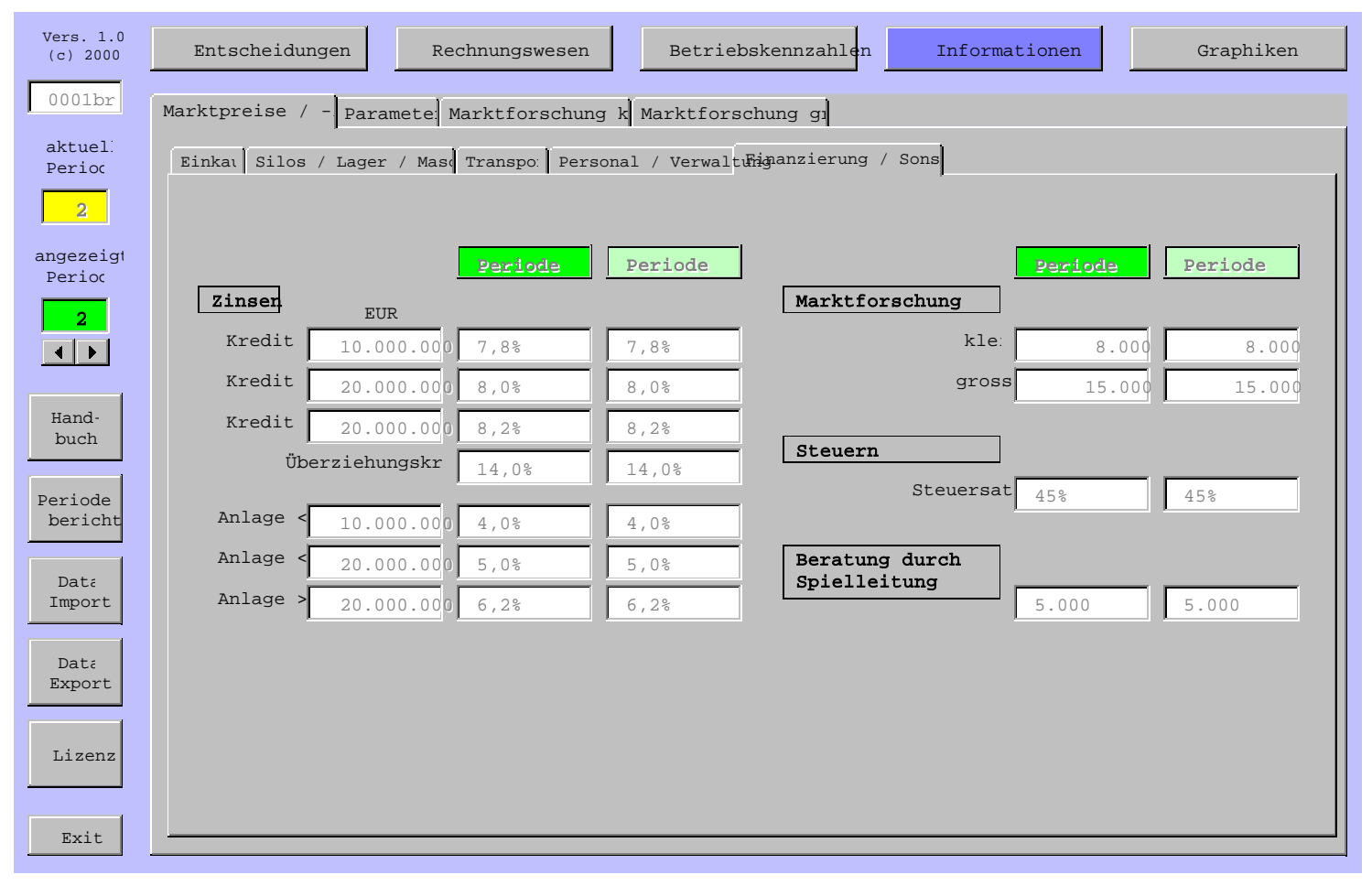

Abb. 60: Ausgabemaske Marktpreise Finanzierung und Sonstiges

Die ausgewiesenen Zinssätze sind Zinsen p.a. In der GuV werden diese entsprechend der Periodenlänge von 2 Monaten um ein Sechstel reduziert.

Die Spielleitung hat die Möglichkeit, den Spielern ein Beratungshonorar in Rechnung zustellen. Dadurch versucht man zu ereichen, dass sie Spieler sich bei Problemen nicht sofort an die Spielleitung wenden. Ziel ist es, dass die Spieler das auftretende Problem eigenständig analysieren und lösen.

\subsubsection{Informationen über Parameter}

Im Unterschied zu den Marktpreisen sind die Parameter in der Regel fix, d.h. sie werden im Verlaufe eines Spieles nicht geändert. Bei den Parametern erhält man Informationen über 
1. Produktion: Stückliste, Gewichte, Volumen, Produktionsdauer etc. der einzelnen Halb- und Fertigfabrikate

2. Maschinen: Kapazitäten, Fix- und variable Produktionskosten, Abschreibungsdauer

3. innerbetriebliche und externe Transporte: benötigte Kapazitäten, Dauer und Volumen sowie über

4. Personal: verfügbare Kapazitäten je Mitarbeitertyp.

Im Menü Stücklisten werden neben detaillierten Stücklisten für Singulus und Combilus Informationen zu Einheiten, technischen Größen oder benötigter Produktionsdauer für jedes der Produkte 1 bis 4 sowie für die beiden VK-Artikel angegeben.

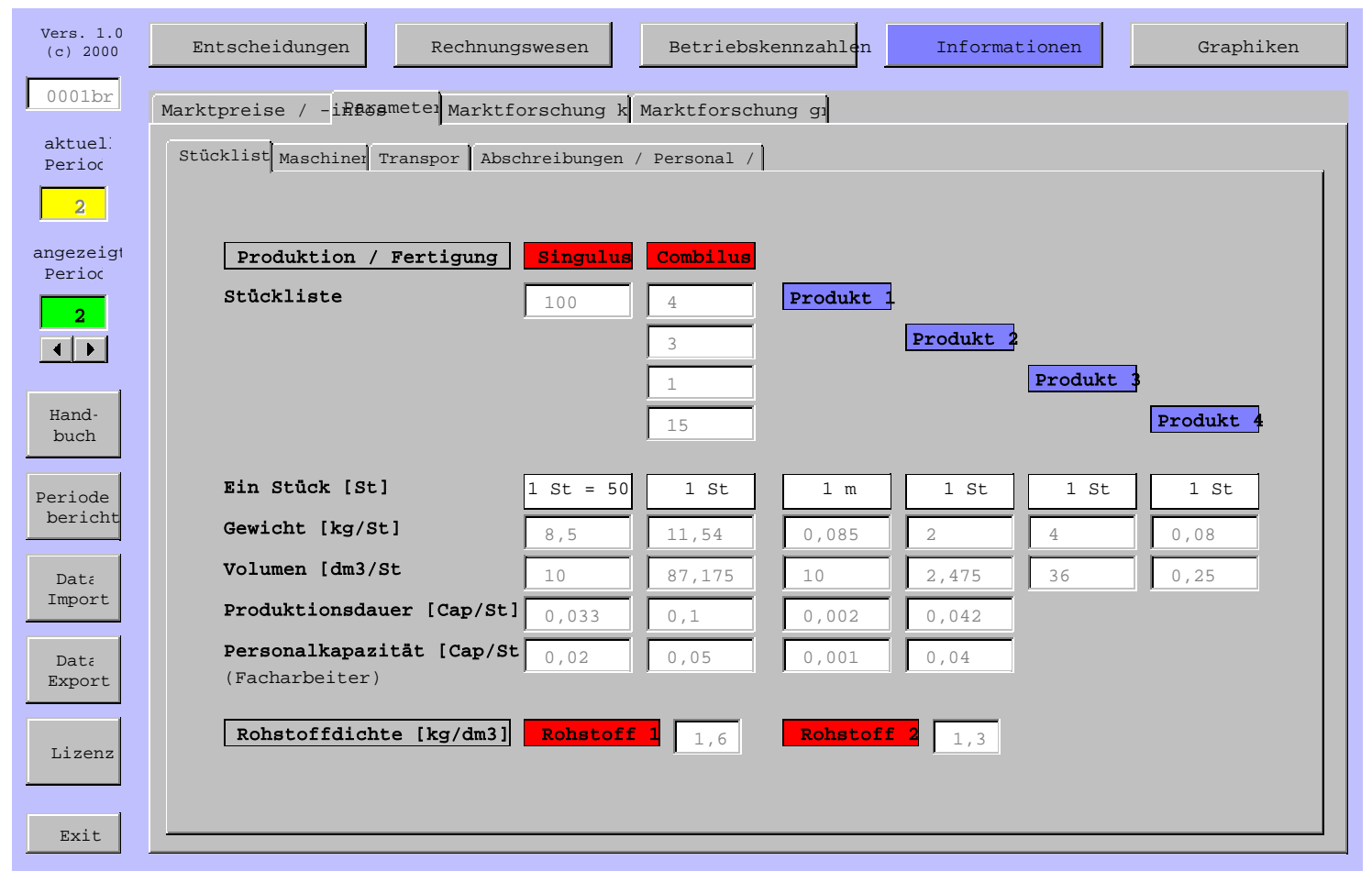

Abb. 61: Ausgabemaske Parameter Stücklisten

Die Stückliste für Combilus setzt sich aus 4 Stück Produkt 1, 3 Stück Produkt 2, 1 Stück Produkt 4 und 15 Stück Produkt 4 zusammen. In der Zeile „Ein Stück“ ist aufgeschlüsselt, welche Einheit „ein Stück“ hat. Im Falle des Produkt 1 ist die z.B. der 1 m, im Falle der anderen drei Produkte jeweils ein Stück. Weiterhin werden Gewichte, Volumen sowie die Produktionsdauer (Produkt 1 und 2) bzw. Montagedauer (Singulus 
und Combilus) der einzelnen Teile angegeben. Die für die einzelnen Schritte notwendigen Personalkapazitäten sind ebenfalls angegeben.

Im Menü Maschinen erhält man technische und wirtschaftliche Informationen zu den benutzten Produktionsmaschinen. So erfährt der Spieler wie hoch die fixen und variablen Kosten einer Periode sind, welche Zuschläge er bei Überstunden auf die variablen Kosten hinzurechnen muss oder über wie viele Perioden die Maschinen abgeschrieben werden.

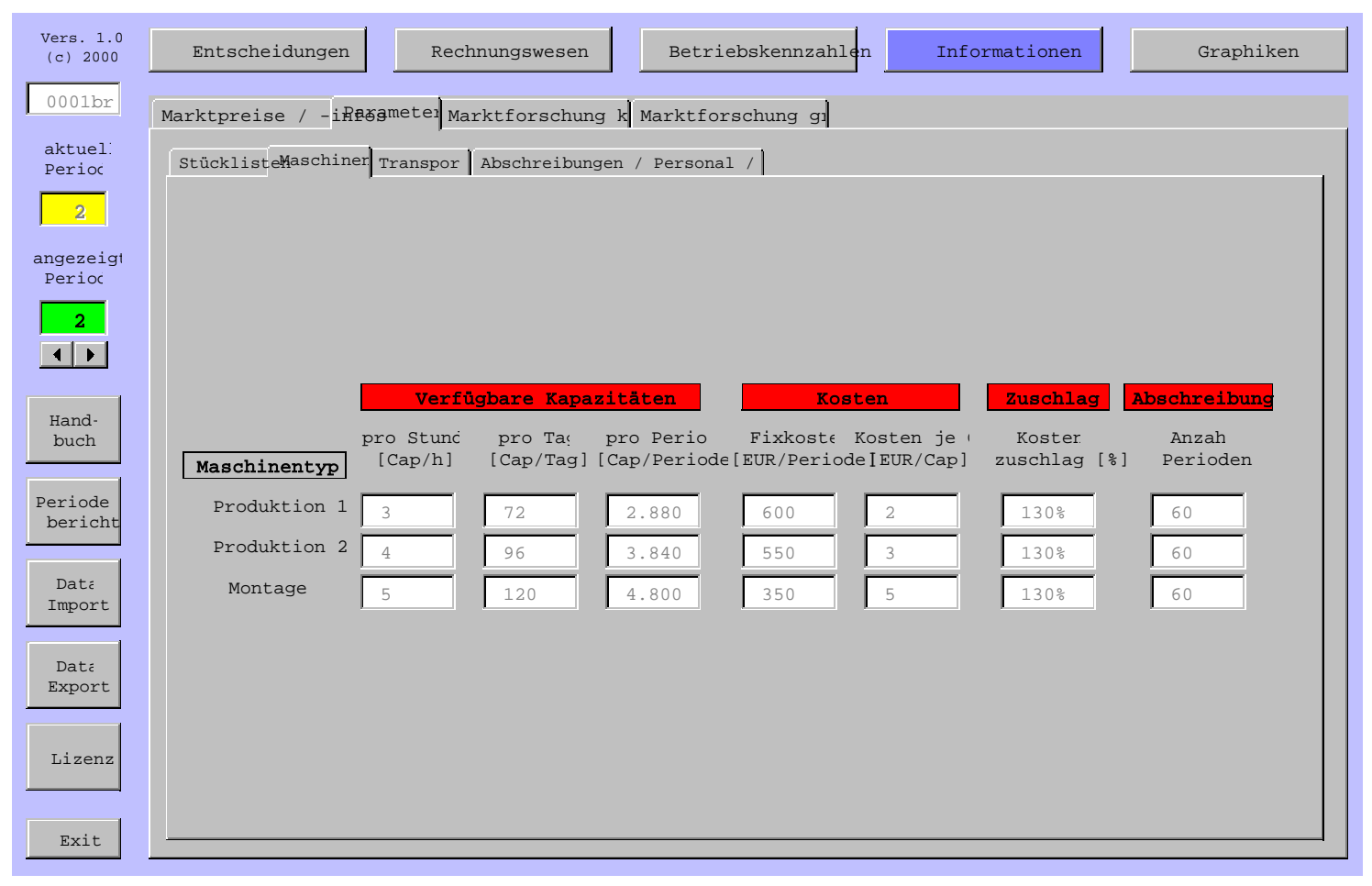

Abb. 62: Ausgabemaske Parameter Maschinen

In obiger Maske gilt für die Maschine der Produktion 1, dass pro Periode Fixkosten in Höhe von $600,-€$ sowie variable Kosten in Höhe von $2 €$ pro produzierter Kapazität anfallen. Verknüpft man die Information 2.880 verfügbare Kapazitäten pro Maschine pro Periode mit der Information, dass die Produktion des Produktes 1 eine Kapazität von 0,002 pro Meter benötigt - diese Informationen findet man in der Maske Stücklisten, die auf der Seite vorher beschrieben wurde - kann man errechnen, dass mit einer Maschine pro Periode 2.880 / 0,002 = 1,44 Mio. m Rohr produziert werden 
können. Dafür fallen variable Betriebskosten von $2 * 2.880=5.760 €$ an. Nicht berücksichtigt werden hier die Material- und Personalkosten.

Im Menü Transporte werden technische (benötigte Kapazitäten Gabelstapler), personelle (benötigte Kapazitäten Hilfsarbeiter) und teilweise wirtschaftliche Parameter (fix und variable Kosten für Gabelstapler) für die innerbetrieblichen Transporte angegeben.

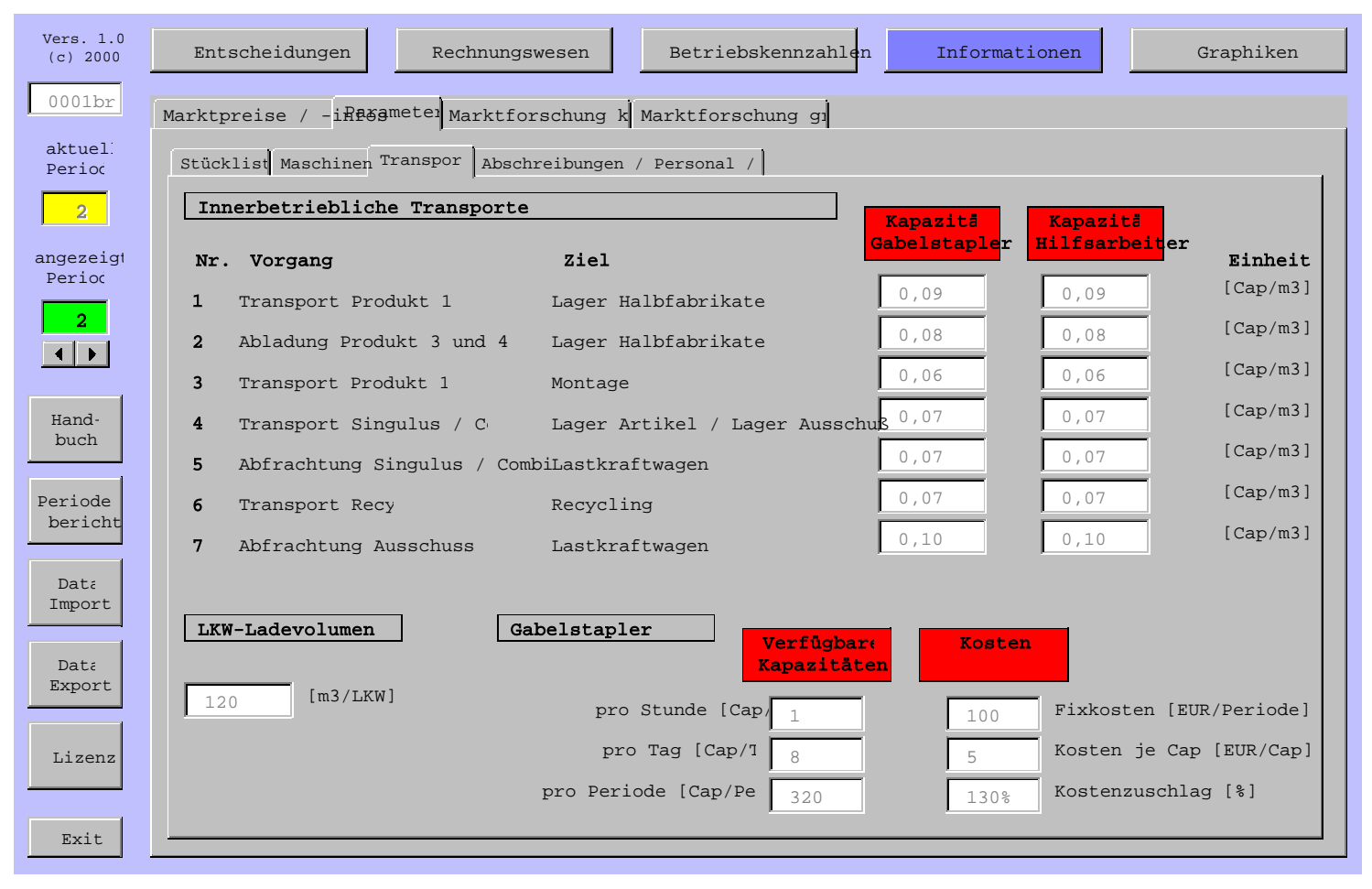

Abb. 63: Ausgabemaske Parameter Transporte

So benötigt z.B. der Transportvorgang Nummer 4 für einen transportierten Kubikmeter eine Gabelstaplerkapazität von 0,07 und eine Personalkapazität von ebenfalls 0,07. Mit der Information über die in einer Stunde verfügbare Gabelstaplerkapazität von 1 lässt sich errechnen, dass ein Gabelstapler in einer Stunde ca. $1 / 0,07 \approx 14,3 \mathrm{~m}^{3}$ der VKArtikel in das Artikellager oder in das Ausschusslager transportieren kann.

Im Menü Abschreibungen / Personal und Kredite kann sich der Spieler über die Abschreibungsdauern des Anlagevermögens, über Kapazitäten der Mitarbeiter sowie über das im in der Periode maximale Kreditvolumen informieren. 


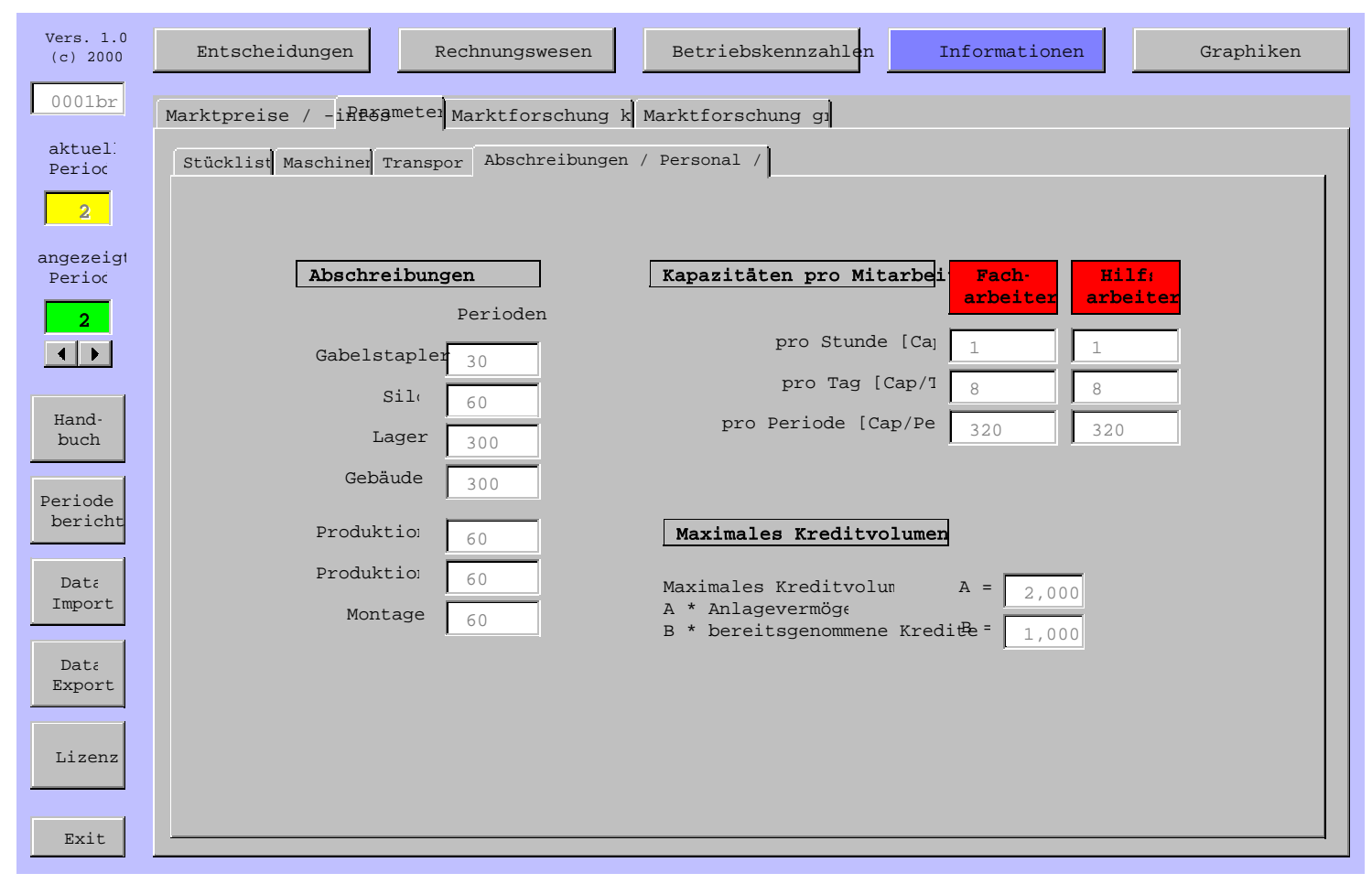

Abb. 64: Ausgabemaske Parameter Abschreibungen, Personal und Kredite

Die Abschreibungsperioden sind durch die Spielleitung auch während eines laufenden Spieles änderbar. Dies bewirkt eine Reduktion des Anlagevermögens.

Bei einem Anlagevermögen von 10 Mio. $€$ und einem bereits in Anspruch genommenen Kredit in Höhe von 4 Mio. $€$ ergibt sich bei obiger Parametereinstellung ein restliches Kreditvolumen von $2 * 10-4=16$ Mio. $€$.

\subsubsection{Ergebnisse der Marktforschung}

Je nach Auswahl der gewünschten Marktforschung durch ein unabhängiges Institut werden bestimmte Informationen den Spieler bereitgestellt. ${ }^{187}$ Hier geht es insbesondere um Marktanteile bei Absatz und Umsatz sowie um die wichtigsten Kennzahlen der Wettbewerber.

187 Die Inhalte sind im Kapitel 4.4.1.1 (Markt- und Wettbewerbsberichte) beschrieben. 


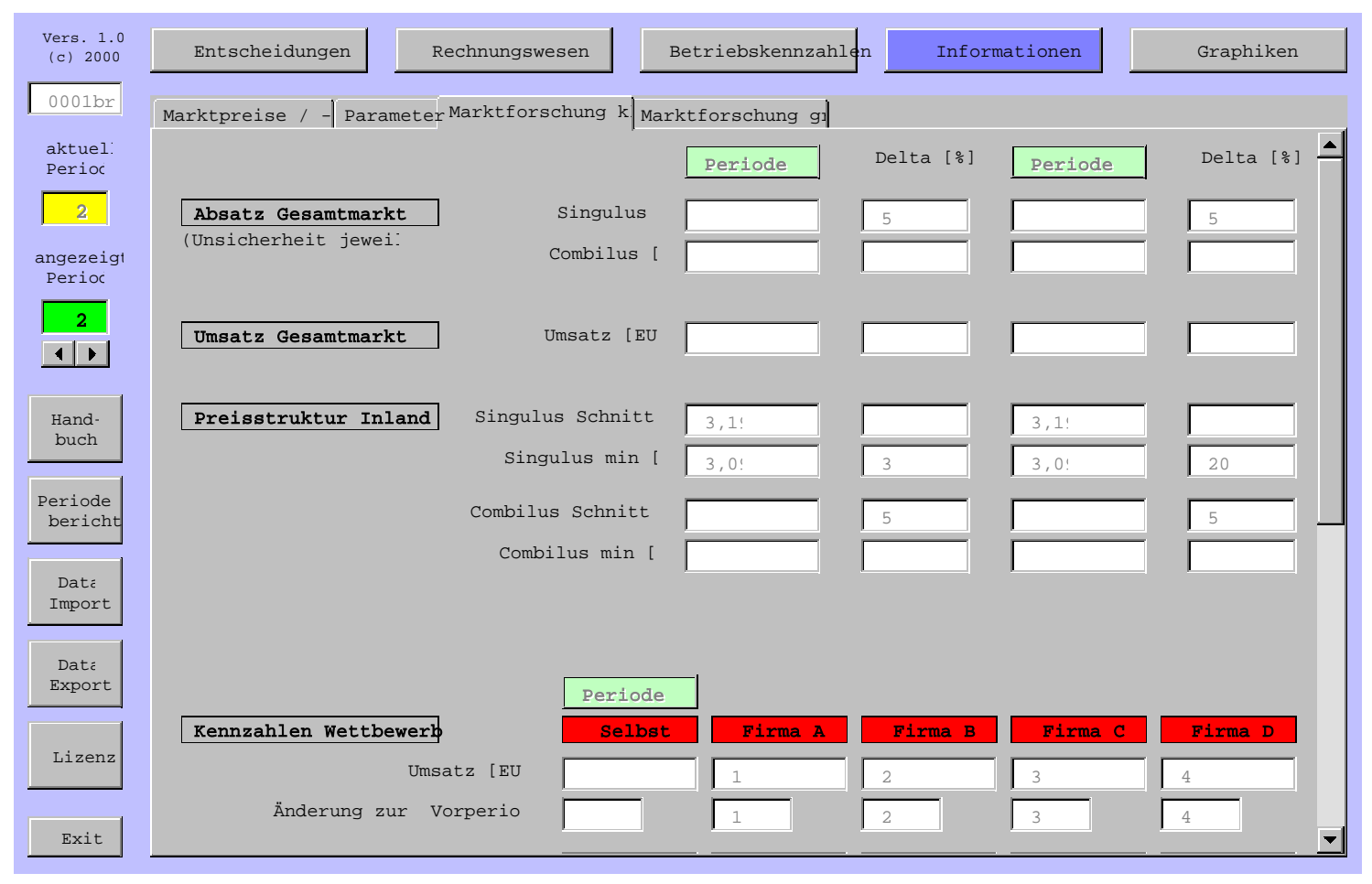

Abb. 65: Ausgabemaske Marktforschung klein (Teil 1)

Der kleine Marktforschungsbericht informiert wie in der obigen Abbildung ersichtlich u.a. über den eigenen Absatzanteil am Gesamtmarkt der beiden Artikel Singulus und Combilus, über den Umsatzanteil aller Spieler am Gesamtumsatz (Singulus und Combilus auf allen Märkten), über die Anzahl der Mitarbeiter sowie über den Perioden- und Gesamterfolg eines jeden einzelnen Bewerbers.

Aufgrund der großen Anzahl der nicht sichtbaren Kennzahlen folgt der untere Teil der Maske Marktforschung klein. 


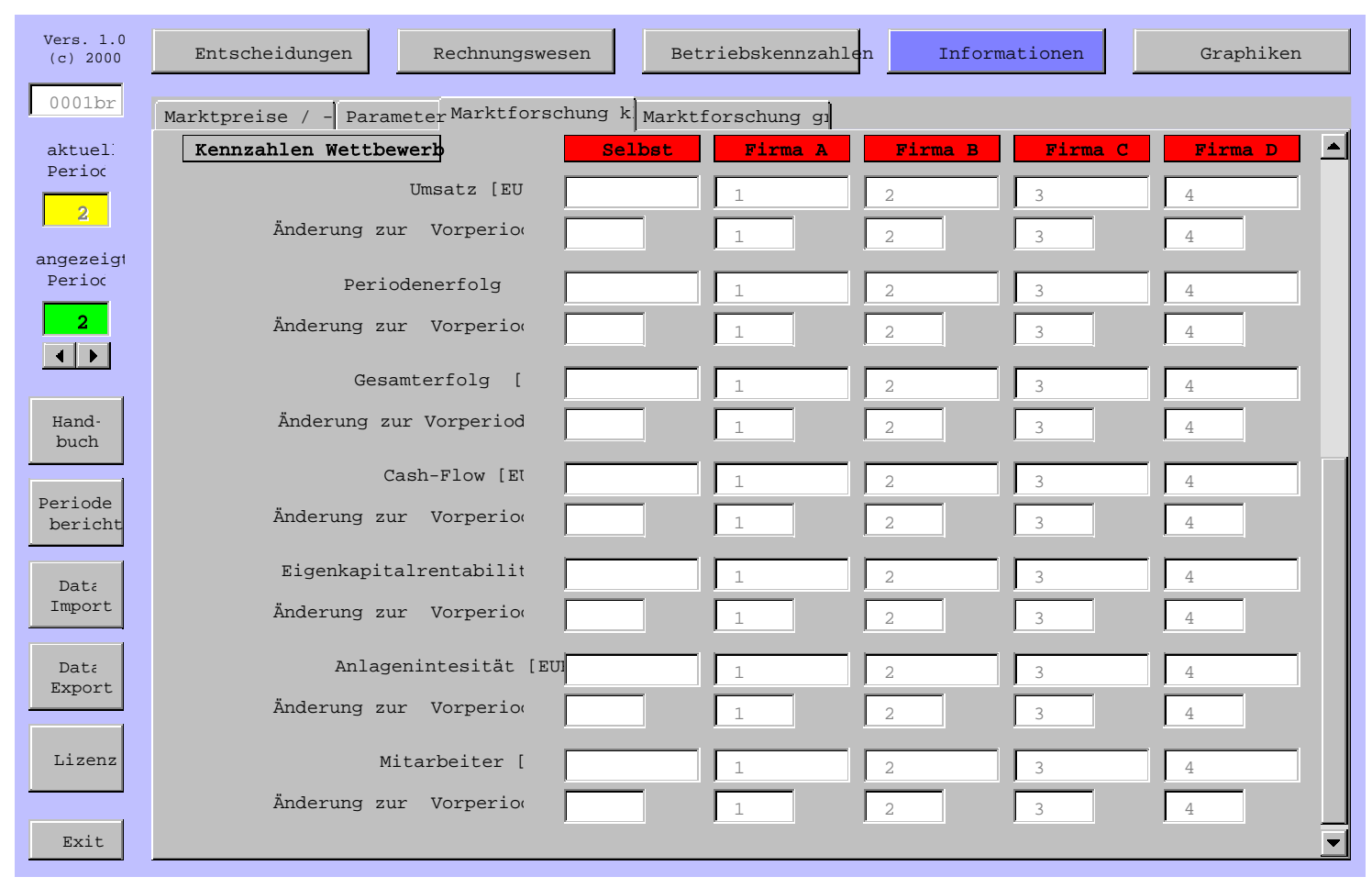

Abb. 66: Ausgabemaske Marktforschung klein (Teil 2)

In dieser Maske sind Informationen ausschließlich aus der vergangenen Perioden dargestellt. Sie sind wie öffentlich zugängliche Geschäftsberichte zu verstehen, die die aktuelle Situation immer nur ,verspätet“ darstellen.

Teile dieser Ergebnisse werden wiederum in der Excel-Datei im Verzeichnis GRAPHICS dargestellt. 


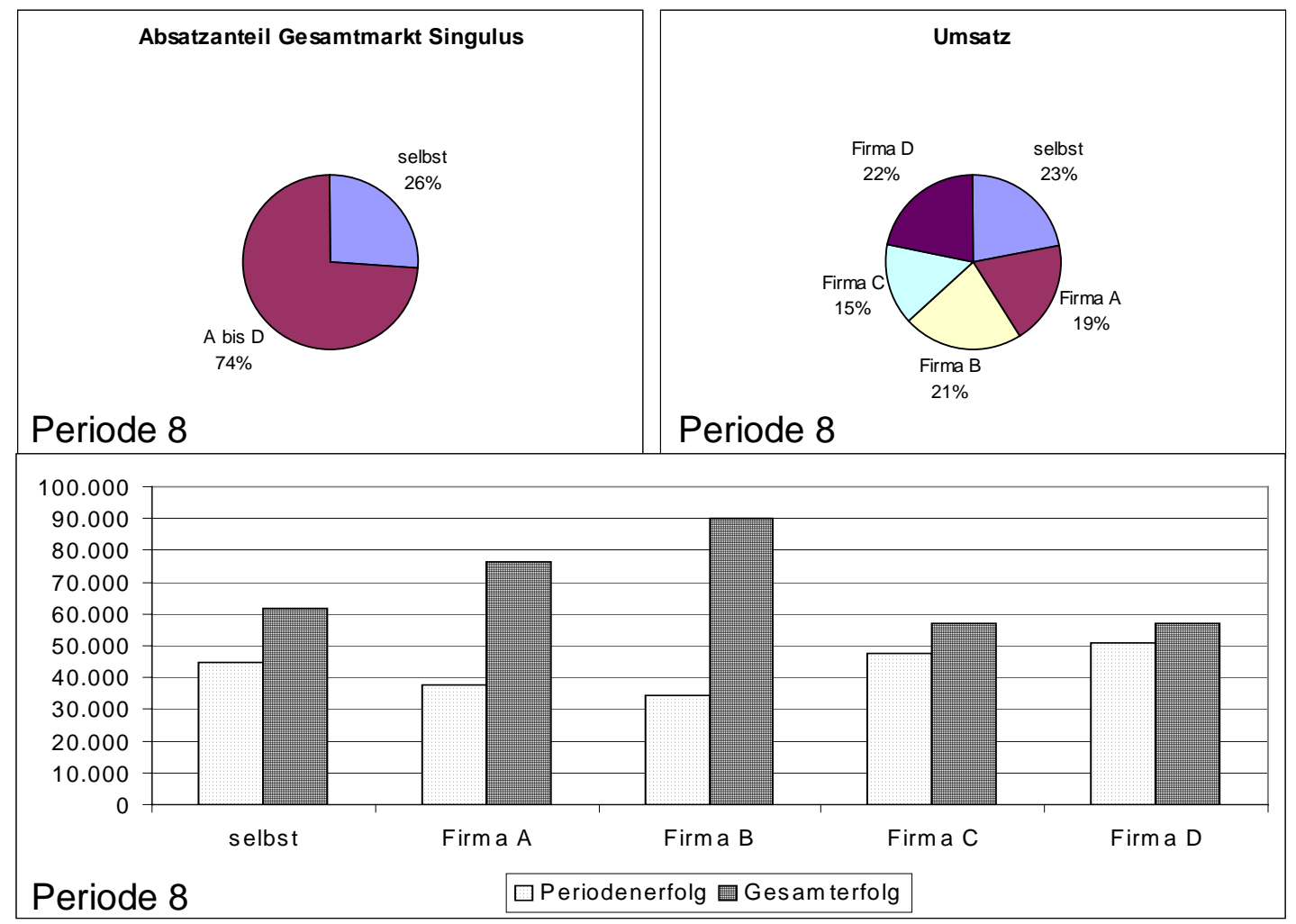

Abb. 67: Auszug Graphische Darstellung Kennzahlen Wettbewerb (Teil 1)

In diesem Bild wird sehr schnell deutlich, wie das eigene Unternehmen gegenüber den Wettbewerbern steht. Der eigene Marktanteil bei Singulus beträgt in diesem Fall 26 Prozent. Somit hat das Unternehmen die anfängliche Startsituation genutzt und den Marktanteil von 20 Prozent auf dem Inlandsmarkt und 8 Prozent auf dem Exportmarkt hervorragend gesteigert. Bezüglich der Umsätze ist das Unternehmen jedoch etwas schlechter positioniert.

Der großen Marktforschungsbericht liefert weitere Informationen über den Markt und die vier Wettbewerber. Neben der Summe der effektiven Werbevolumen - gemäß Definition sind dies die Werbeausgaben über drei Perioden verteilt - finden sich Informationen über die Preisstruktur sowie detaillierte Kennzahlen über die Wettbewerber. 


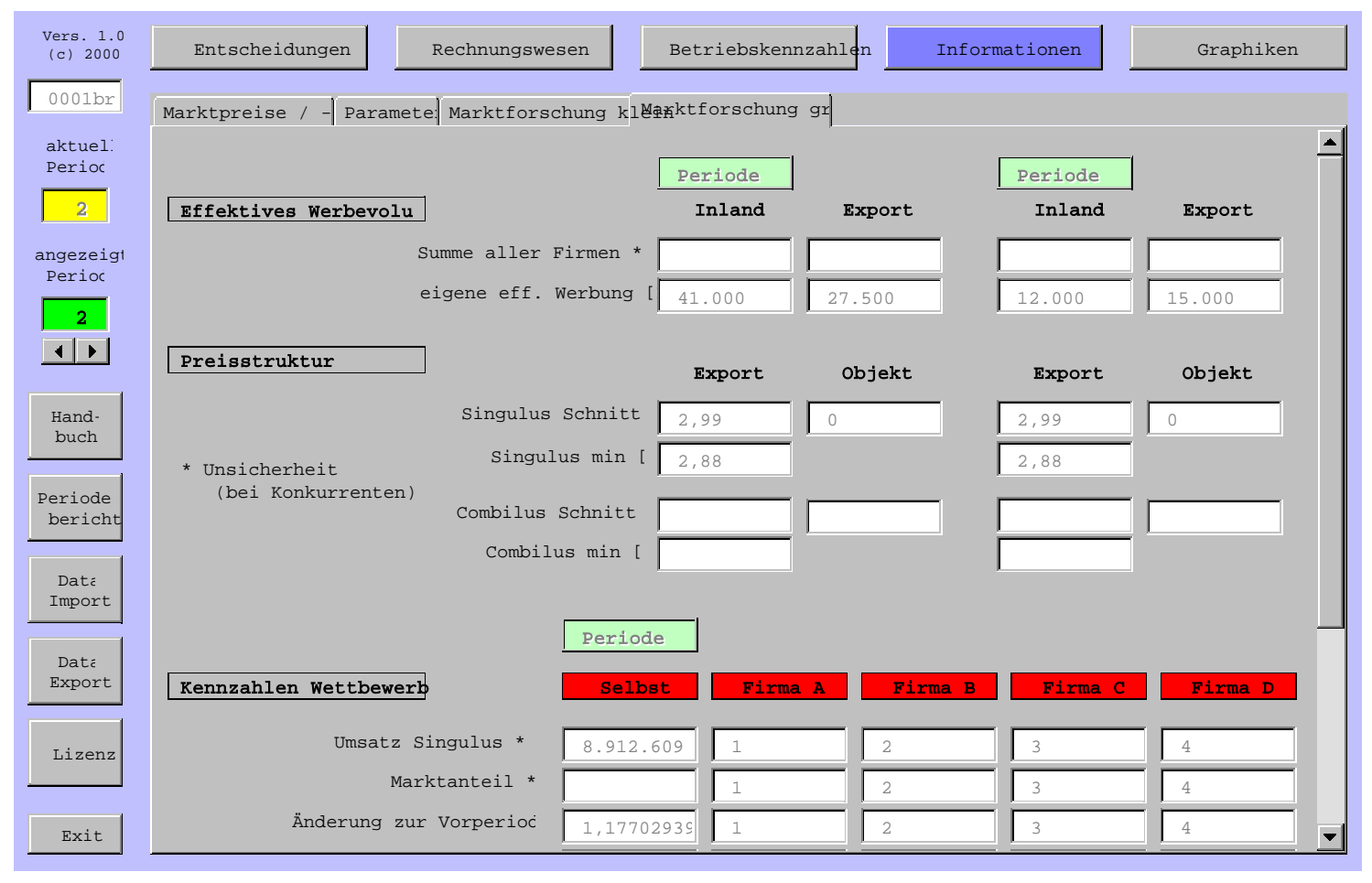

Abb. 68: Ausgabemaske Marktforschung groß (Teil 1)

Aufgrund der großen Anzahl der nicht sichtbaren Kennzahlen folgt der untere Teil der Maske Marktforschung groß. 


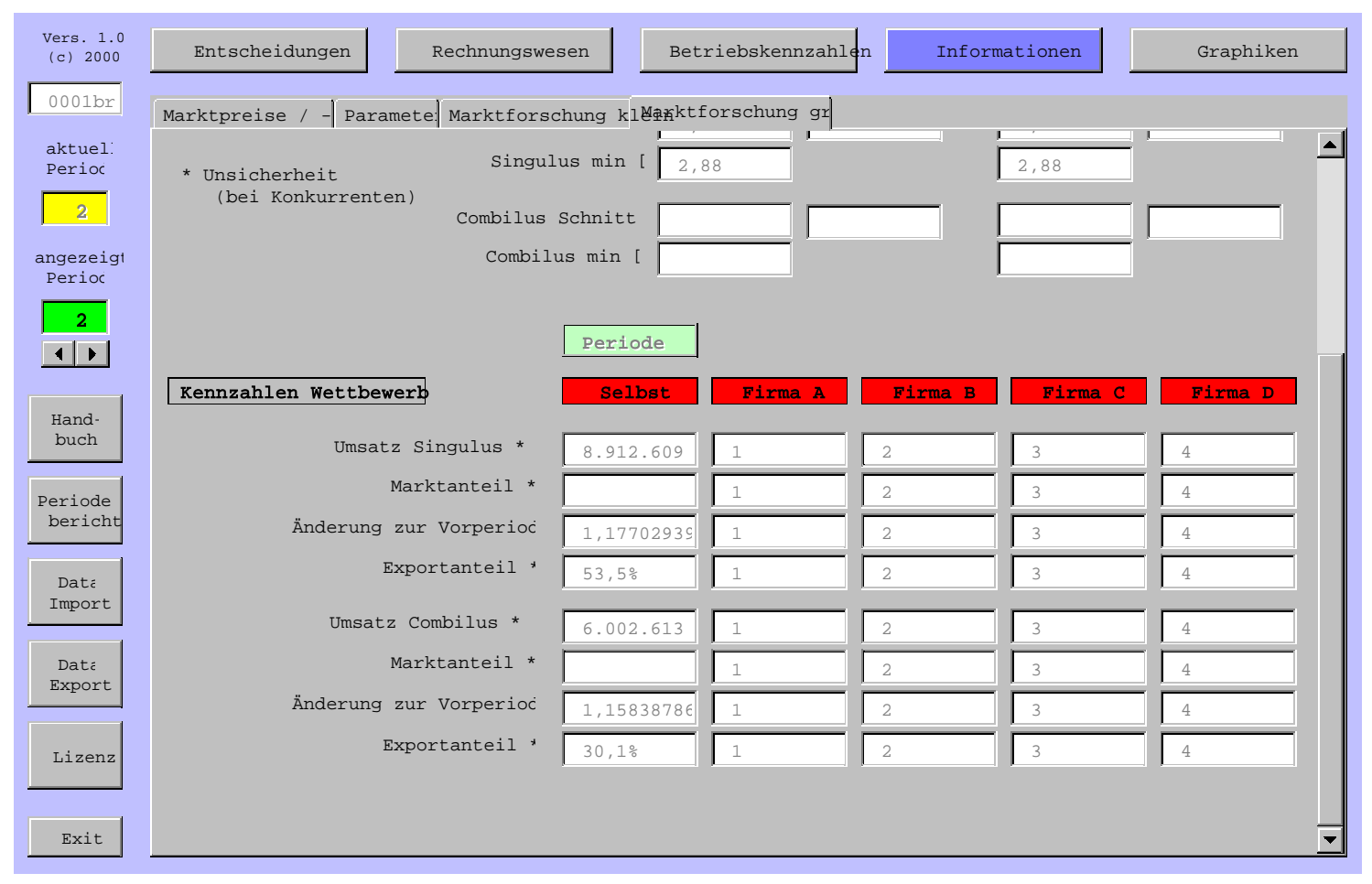

Abb. 69: Ausgabemaske Marktforschung groß (Teil 2)

Die große Marktforschung liefert deutlich weitergehende Ergebnisse als die kleine Marktforschung. Hier werden u.a. Zahlen der jeweiligen Umsatzträger, Markt- und Exportanteile angegeben. 


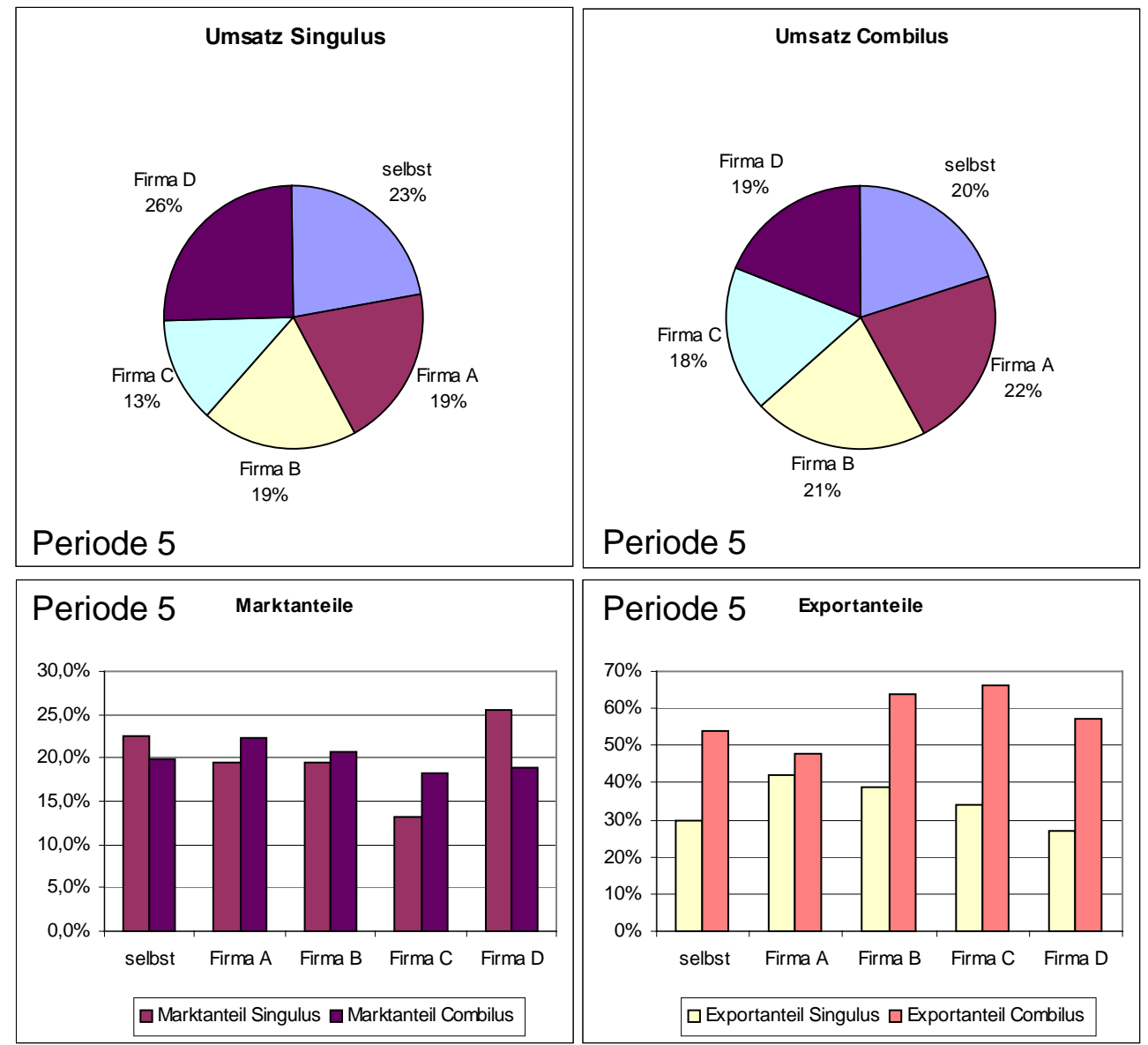

Abb. 70: Auszug Graphische Auswertung Kennzahlen Wettbewerb (Teil 2)

In obiger Darstellung ist ersichtlich, dass das Unternehmen beim Umsatz Singulus über dem Marktdurchschnitt von 20 Prozent liegt (Bild links oben). Beim Umsatz Combilus liegt das Unternehmen genau im Marktdurchschnitt (Bild oben rechts). Diese beiden Bilder sind links unten zusammengefasst. Deutlich erkennt man, dass das Unternehmen C das Schlusslicht bildet. 


\subsection{Reihenfolge der Simulation}

Das Programm selbst ist in verschiedene Module eingeteilt. Diese werden in folgender Reihenfolge gerechnet:

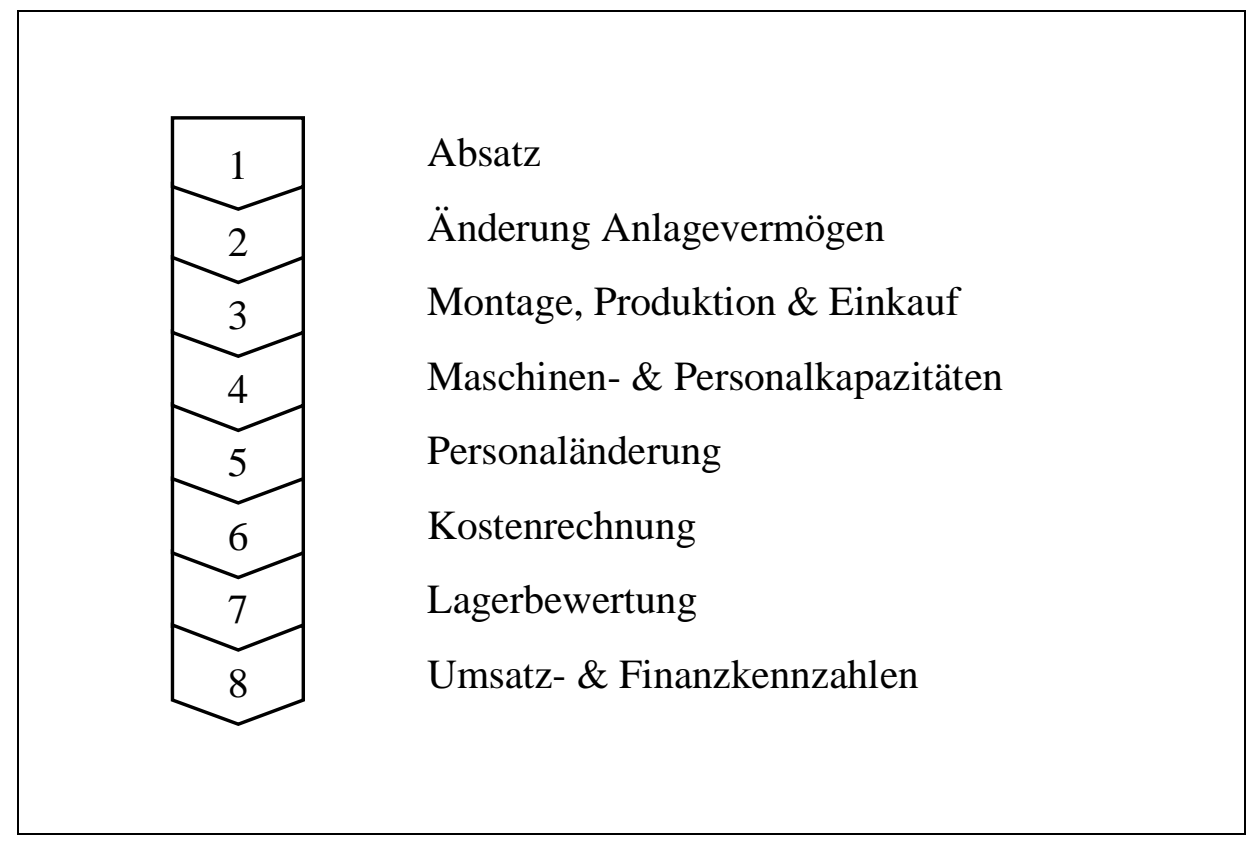

Abb. 71: Reihenfolge der Simulation

\section{Anmerkungen:}

1. Absatz: In der Planungsphase jedes einzelnen Unternehmens - d.h. vor der Bestätigung der Absatzplanung - werden alle Eingaben der Spieler als Soll-Werte übernommen. So gilt für die direkt daraus folgenden Kennzahlen wie Bilanz, GuV, Lager- und Produktionszahlen, dass die Planmengen auch wirklich zu dem gewünschten Preis abgesetzt wurden.

Nachdem die Spieler ihre Planungen an den Marktplatz geschickt haben, werden dort die definitiven Absatz- und Preispotenziale bestimmt (Marktphase). Diese werden dann den Spieler zugewiesen. Daraus resultieren die definitiven Absatzmengen und Preise, was wiederum Einfluss auf Produktion, GuV, Bilanz, Kasse, etc. hat. 
2. Anlagevermögen: Die gewünschten notwendigen Änderungen bei den Lagern, den Silos und den Produktionsmaschinen werden durchgeführt.

3. Montage, Produktion und Einkauf: Nachdem bereits unter (1) erfolgten Absatz schließt sich die Montage bzw. Produktion an. Dabei wird das Schaubild aus Abb. 4 (Leistungsfluss des Unternehmens LogisticPLUS) von rechts nach links „abgearbeitet“: Das freie Volumen im VK-Lager hat sich aufgrund des realisierten Absatzes vergrößert. Dadurch können die montierten Verkaufsartikel Singulus und Combilus eingelagert werden. Dies hat zur Folge, dass das Halbfabrikat-Lager freies Volumen erhält, was wiederum für die Einlagerung der Artikel 1 bis 4 Voraussetzung ist.

Anmerkung: Aufgrund der Prüfung der einzelnen Aufträge nacheinander kann es vorkommen, dass z.B. das Volumen des Halbfabrikat-Lagers für die zu produzierenden Volumen Produkt 1 und 2 sowie für die einzukaufenden Volumen Produkt 3 und 4 nicht ausreicht. Bevor dies überprüft wird und gegebenenfalls die Bestell- bzw. Produktionsmengen dementsprechend verringert werden, wird festgestellt, ob die Produkte 1 und 2 überhaupt in der gewünschten Menge produziert werden können. Hier erfolgt wenn notwendig auch eine Verringerung der Produktionszahlen.

4. Kapazitäten: Die für die Produktion und Montage benötigten Maschinen- und Personalkapazitäten werden berechnet. Waren zuwenig Kapazitäten vorhanden, werden teurere Leihkapazitäten in Anspruch genommen.

5. Personaländerung: Es werden Fach- und Hilfsarbeiter eingestellt oder entlassen. Diese haben somit keinen Einfluss auf die Wertschöpfung in der aktuellen Periode. Allerdings fallen bereits Kosten für Einarbeitung an.

6. Kostenrechnung: liefert u.a. die Herstellungskosten der Artikel Singulus und Combilus. Diese werden als Bilanzwerte übernommen.

7. Lagerbewertung: Berechnung und Bewertung der Bestände, Reichweiten der Lager.

8. Umsatz- und Finanzkennzahlen: Bilanz, GuV, Cashflow, Umsatzrentabilität und Liquidität werden berechnet. Sollte das bisherige Fremdkapital nicht ausreichen, um die Liquidität sicherzustellen, kommt es zur Vergabe eines teuren Liquiditätskredites. Dies wird dem Spieler angezeigt. Sollte ein Liquiditätskredit in Anspruch genommen werden, folgen daraus Änderungen in GuV und Bilanz. 


\section{Konzeption des Spielleitermoduls}

Game participants learn from their own results as well of the results of others. 188

\subsection{Aufbau und Bedienung}

Das Spielleiter-Modul hat drei unterschiedliche Aufgaben:

1. Einerseits soll das Modul den Spielleiter insbesondere bei der Analyse der vergangenen Spielrunde und somit bei der periodischen Erstellung der „Berichte der Spielleitung“ unterstützen. In diesen Berichten werden Informationen zum derzeitigen und zukünftigen Marktgeschehen an alle Spieler verteilt. Dies gilt auch für notwendige Detailanalysen für einzelne Spieler, wenn diese „,mit ihrem Latein am Ende sind“.

2. Es soll andererseits die Märkte simulieren, auf denen die Produkte abgenommen werden bzw. auf denen die Nachfrage vorhanden ist. Ergebnis der Simulation sind z.B. Zuteilungen von maximal möglichen Absatzvolumen der beiden Produkte sowie die Bestimmung der Unterstützungsfaktoren (z.B. des Werbefaktor).

3. Zuletzt hat der Spielleiter die Möglichkeit, für die nächste Periode verschiedenste Parameter zu beeinflussen. Er kann beispielsweise ankündigen, dass die Nachfrage in den nächsten Perioden abnehmen wird oder dass bestimmte Rohstoffe teurer werden.

Sieht man sich die im Kapitel 4.1 gezeigte Abbildung „Abwicklung einer Periode“ aus der Sicht der Spielleitung an, so ergibt sich nachfolgendes Bild.

188 Anderson / Morrice (Simulation Game, 2000) Seite 40. 


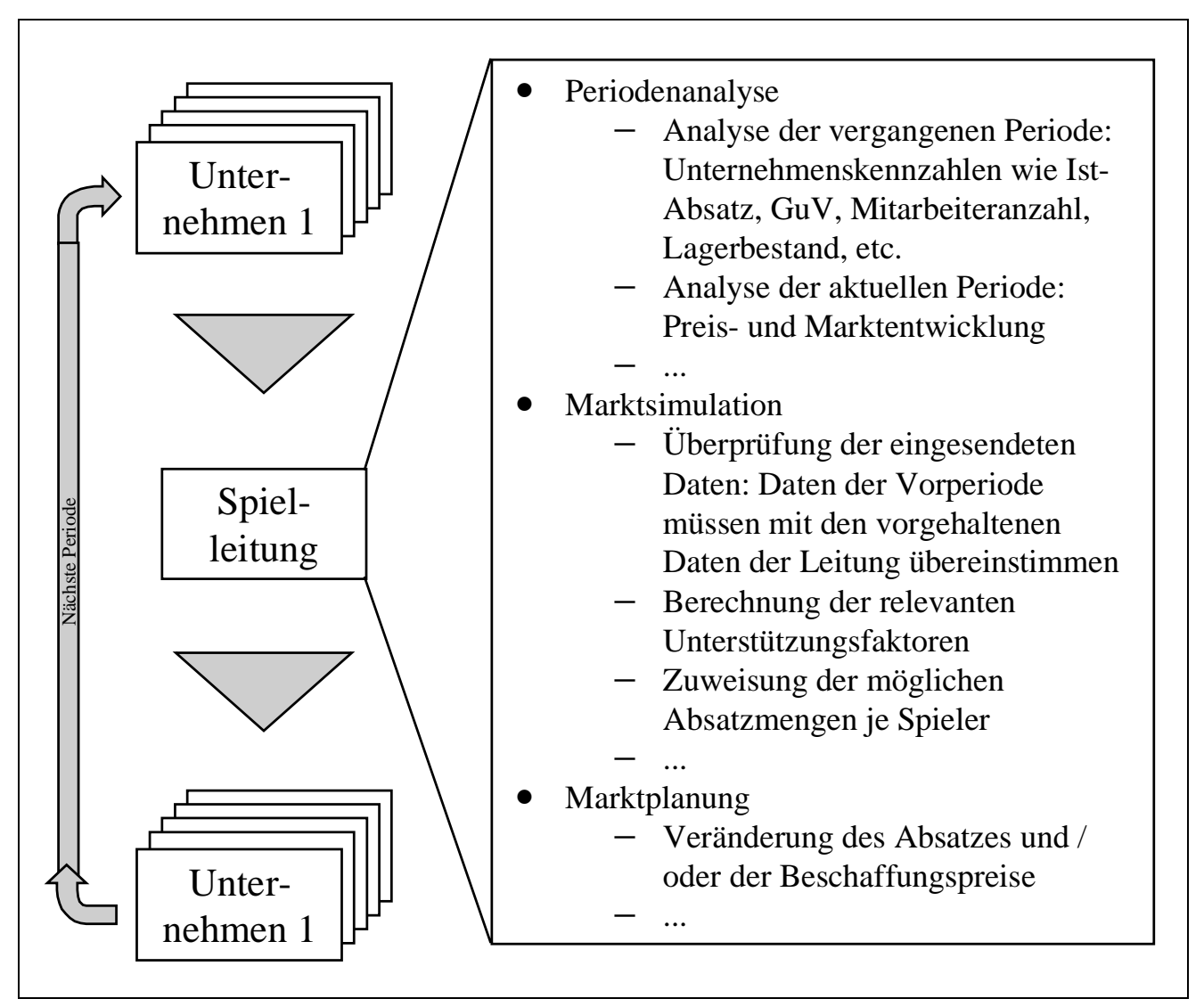

Abb. 72: Abwicklung der Simulation

Die Unternehmen planen als erstes ihre Entscheidungen z.B. der Periode T= 3 auf Basis der Ergebnisse der vorhergehenden Periode $(\mathrm{T}=2)$. Nach der Versendung der Daten an die Spielleitung werden im Spielleiterprogramm drei Schritte durchlaufen:

1. In der Periodenanalyse wird die vergangene Periode $(T=2)$ analysiert. Dabei wird u.a. ausgewertet, welche Marktanteile die Unternehmen wirklich erzielt haben oder welche internen und externen Kennzahlen sich für die Unternehmen aufgrund ihrer und der Entscheidungen der vier Konkurrenten ergeben haben.

2. In der Marktsimulation wird die aktuelle Periode ( $T=3)$ simuliert. Es werden z.B. die eingesendeten Daten überprüft sowie aufgrund der Entscheidungen der einzelnen Spieler ihnen Marktanteile zugewiesen.

3. Die Marktplanung widmet sich den folgenden Perioden $(\mathrm{T} \geq 4)$. Hier kann die Spielleitung Einfluss auf die Parameter nehmen und so z.B. die Preise für Beschaffung ändern oder aber das Marktvolumen nach oben oder unten korrigieren. 
Das Spielleiter-Modul ist optisch ähnlich dem Spieler-Modul aufgebaut. Auch hier stehen verschiedene Funktionen stehen zur Verfügung:

1. Data Import: Es werden die Daten der fünf Spieler eingelesen. Dabei werden die importierten Daten mit dem der Spielleitung vorliegenden Stand verglichen. Sollten Abweichungen (Manipulation) erkannt werden, wird dies dem Spielleiter mitgeteilt.

2. Simulation / Graphiken: Hier findet die Berechnung der neuen Nachfragemengen der Märkte je Spieler und Produkt statt.

3. Neue Periode: Es werden die Vorgabewerte der aktuellen Periode in die neue Periode übernommen. Gegebenenfalls bereits vorab geänderte Felder werden nicht überschrieben. Es erfolgt eine Mitteilung an den Bediener.

4. Neuer Bericht: Ein HTML-File wird aufgerufen.

5. Öffne Bericht: Es wird der Bericht der angezeigten Periode geöffnet.

6. Data Export: Die Daten der Spieler werden in EXCEL-Files kopiert und in einem Datenverzeichnis der aktuellen Periode abgelegt.

7. Vorgaben Spielleitung: Die Spielleitung kann hier verschiedene Parameter vorgeben oder ändern.

8. Analyse Gesamtmarkt: Auswertung der Märkte, jeweils Summenbetrachtungen.

9. Einzelanalyse Spieler: Verschiedene Kenngrößen der einzelnen Spieler werden dargestellt.

10. Daten zum Spiel: Teilnehmerinformationen (Namen, Kontakt- und sonstige Daten).

\subsection{Steuerungsgrößen}

Die wichtigsten Vorgaben der Spielleitung sind die Nachfragefunktionen der beiden Produkte auf den einzelnen Märkten. Die Spielleitung gibt für jedes Produkt auf dem Inlands- und dem Exportmarkt die preisabhängigen Nachfragefunktionen vor. Dazu legt die Spielleitung die jeweilige Nachfragemenge bei zwei Preisen fest. 


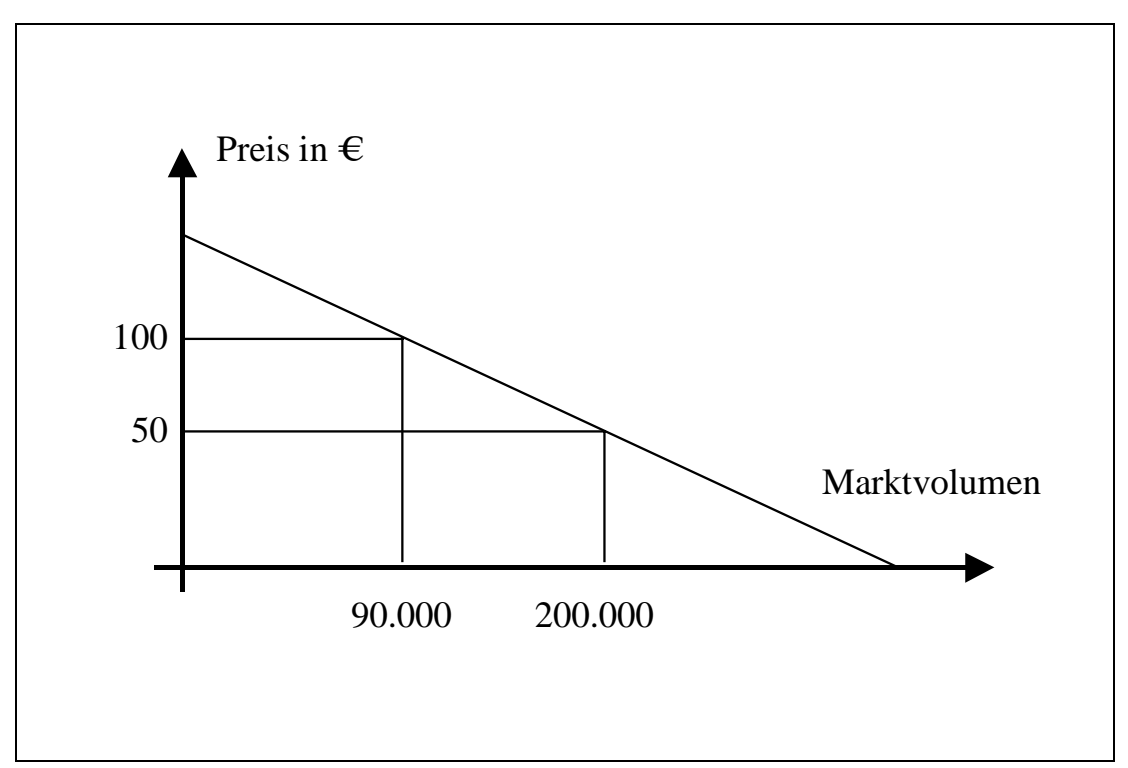

Abb. 73: Nachfragevorgabe durch die Spielleitung

Der Spielleiter muss vor der ersten Runde zwei Preise und die damit verknüpften Nachfragemengen des Marktes vorgeben. Der erwartete Durchschnittspreis ${ }^{180}$ sollte dabei irgendwo in der Mitte der beiden Preisvorgaben liegen. In obigem Beispiel werden somit bei einem durchschnittlichen Marktpreis von 100,- $€ 90.000$ Stück eines Produktes nachgefragt. Da die Funktion linear verläuft, würden bei einem Preis von 75,$€ 145.000$ Stück nachgefragt werden.

Neben der direkten Einflussnahme auf jedes einzelne Unternehmen - die Daten der Spieler können im Falle von Fehlern, Liquiditätsengpässen oder sonstigen Punkten nachträglich abgeändert werden - besteht für die Spielleitung die Möglichkeit, eine Reihe von generellen Parametern zu ändern. Nachfolgend eine Aufstellung über die beeinflussbaren Größen.

189 Die Spielleitung erhält in deren Hilfsprogramm detaillierte Hinweise, in welcher Bandbreite die Selbstkosten der Spieler bei welcher Startsituation jeweils liegen können. Je nach langfristiger Marktplanung der Spielleitung (Schrumpfung, Wachstum, etc.) kann die Nachfragemenge bzw. die Bruttomarge für die Spieler mittels der Nachfragemenge über dem Preis ,eingestellt“ werden. 


\begin{tabular}{|l|l|}
\hline Bereich & Parameter \\
\hline Nachfrage & $\begin{array}{l}\text { Festlegung der Nachfrage für zwei Produkte auf zwei Märkten } \\
\text { Festlegung des Ausschreibungsvolumens und Mindestpreises }\end{array}$ \\
\hline Einkauf / Entsorgung & $\begin{array}{l}\text { Rohstoff- und Produktpreise } \\
\text { Kosten für Recycling } \\
\text { Kosten für Rücknahme der zuviel bestellten Rohstoffe oder Produkte }\end{array}$ \\
\hline Silos / Lager & $\begin{array}{l}\text { Preise für neu zu beschaffende Silos oder Lagerhallen } \\
\text { Bestimmung der zu erwartenden Mieteinnahme bei Vermietung }\end{array}$ \\
\hline Maschinen & $\begin{array}{l}\text { Preise für neu zu beschaffende Maschinen } \\
\text { Festlegung der fixen und variablen (Produktions-) Kosten je Maschinentyp }\end{array}$ \\
\hline Transporte & $\begin{array}{l}\text { LKW-Transport- und Anfahrtsgebühren } \\
\text { Preise für neu zu beschaffende oder zu mietende Gabelstapler }\end{array}$ \\
\hline Personal & $\begin{array}{l}\text { Lohnkosten je Mitarbeitertyp } \\
\text { Einstellungs- und Entlassungskosten } \\
\text { Zuschlagsfaktor bei Überstunden }\end{array}$ \\
\hline Finanzen / Sonstiges & $\begin{array}{l}\text { Festlegung der Zinsaufwendungen (Zinserträge) bei Krediten (Anlagen) } \\
\text { Kosten für Marktforschung } \\
\text { Kosten für Beratungsdienstleistungen }\end{array}$ \\
\hline
\end{tabular}

Tab. 43: Auswahl Steuerungsgrößen des Spielleiters

Die Parameter sind einzeln durch die Spielleitung änderbar. Um die Einfachheit der Bedienung durch die Spielleitung zu gewährleisten besteht die Möglichkeit, dass per automatisiertem Befehl alle Parameter der aktuellen Periode in die folgende Periode übernommen werden. Eventuell bereits vorab geänderte Parameter - z.B. eine in Periode 3 angekündigte Ausschreibung für Periode 6, die damals auch bereits in das Spielleitermodul eingetragen wurde - wird nicht überschrieben. Der Spielleiter erhält in diesem Fall einen Hinweis, dass bestimmte Parameter bereits Werte enthalten haben. 


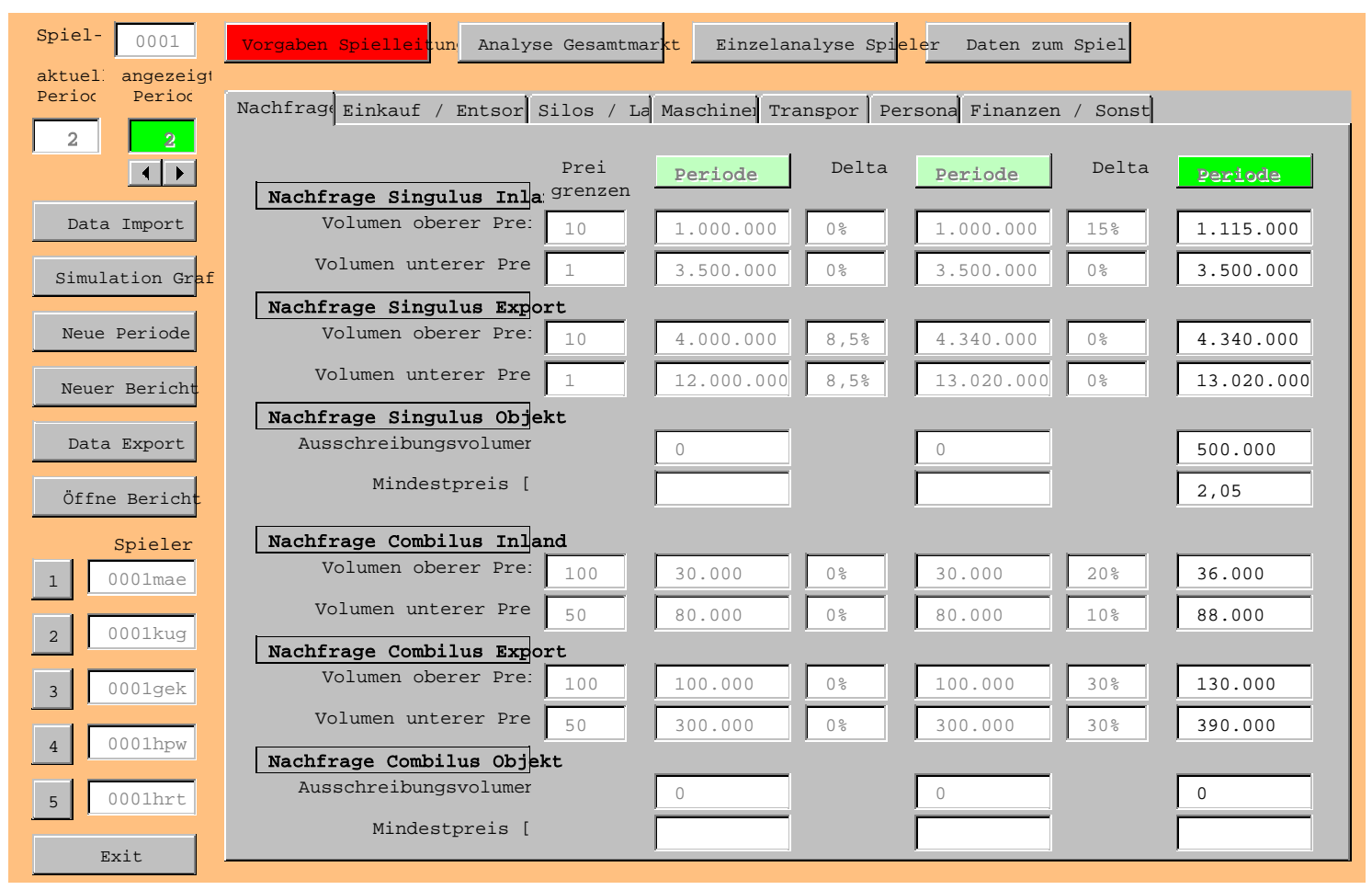

Abb. 74: Einstellungsmöglichkeiten Nachfrage (Spielleitung)

Das obige Bild zeigt eine Maske der zahlreichen Einstellmöglichkeiten für die Spielleitung. Für die beiden Produkte Singulus und Combilus können jeweils die beiden preisabhängigen Volumen für den Inlands- und den Exportmarkt angegeben werden. Dabei wird wie oben beschrieben eine Nachfragefunktion mit zwei Preisen und zwei dazugehörigen Mengen vorgegeben. Weiterhin hat die Spielleitung die Möglichkeit Ausschreibungen für die einzelnen Produkte anzukündigen. In Periode 2 werden z.B. $500.00 \mathrm{~m}$ Singulus zu einem Mindestpreis von 2,05€ angeboten.

\subsection{Auswertungen}

Dem Spielleiter hat einerseits die Möglichkeit, eine Einzelbetrachtung bei jedem Spieler durchzuführen. Somit hat er dieselben Auswertungen, wie sie sich dem Spieler bieten. Die Darstellungen entsprechen den im Kapitel 4 beschriebenen Graphiken. 
Andererseits werden automatisch Gesamtauswertungen berechnet. Diese Auswertungen sind Aggregationen über alle fünf Spieler Diese werden in ihrer Gesamtheit nur dem Spielleiter zur Verfügung gestellt. ${ }^{.90}$ Nachfolgend zwei repräsentative Beispiele.

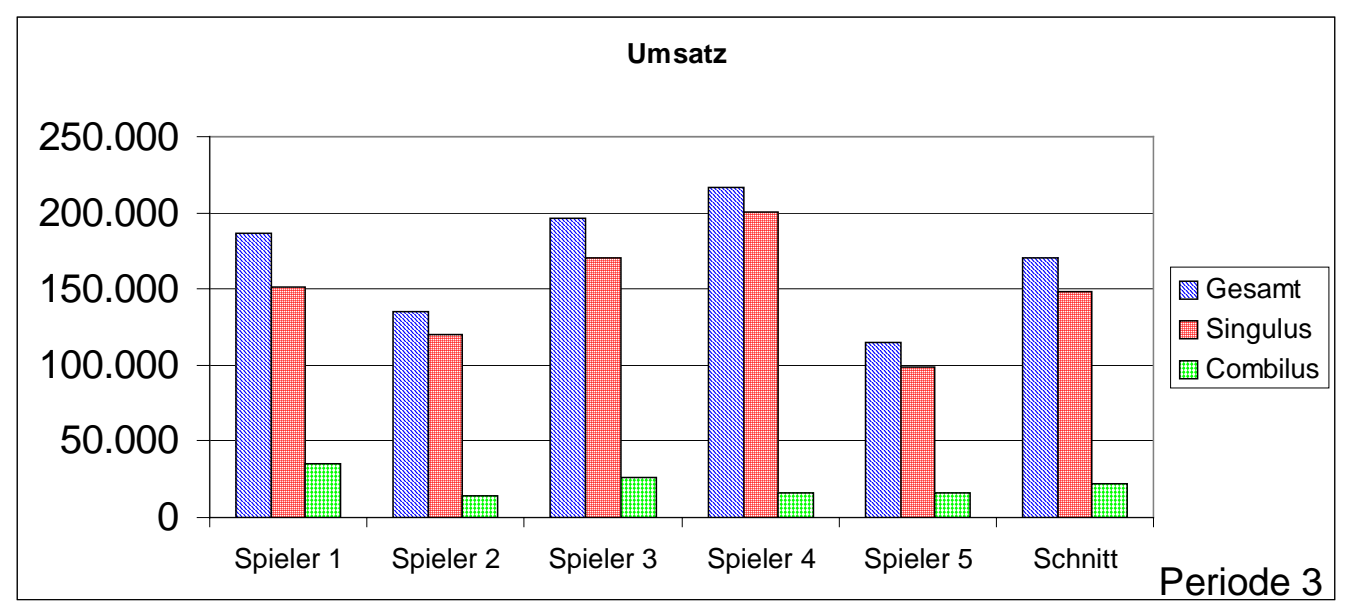

Abb. 75: Spielleiterauswertung Umsatz

In nachfolgendem Bild wird deutlich, welcher Einfluss durch die Investitionen auf den Absatz ausgeübt wird.191 Man kann gut erkennen, wie die einzelnen Spieler ihren aufgrund des Preises zugewiesenen möglichen Absatz (Marktanteil) aufgrund getätigter Investitionen vergrößern oder verkleinern. Hierbei ist zu beachten, dass einige Faktoren auch durch die Entscheidungen der Wettbewerber beeinflusst werden.

190 Die Spieler haben jedoch die Möglichkeit, zu bestimmten Kosten Teile dieser Auswertungen als Marktforschungsberichte zu kaufen.

191 So wird beispielsweise beim Werbefaktor Inland der Spieler 1 mit dem Wert 1,2 unterstützt. Dies bedeutet, dass der aufgrund des Preises ihm zugewiesene Marktanteil mit dem Wert 1,2 multipliziert wird. Siehe hierzu auch Kapitel 4.3.1.3 (Absatzunterstützung). 


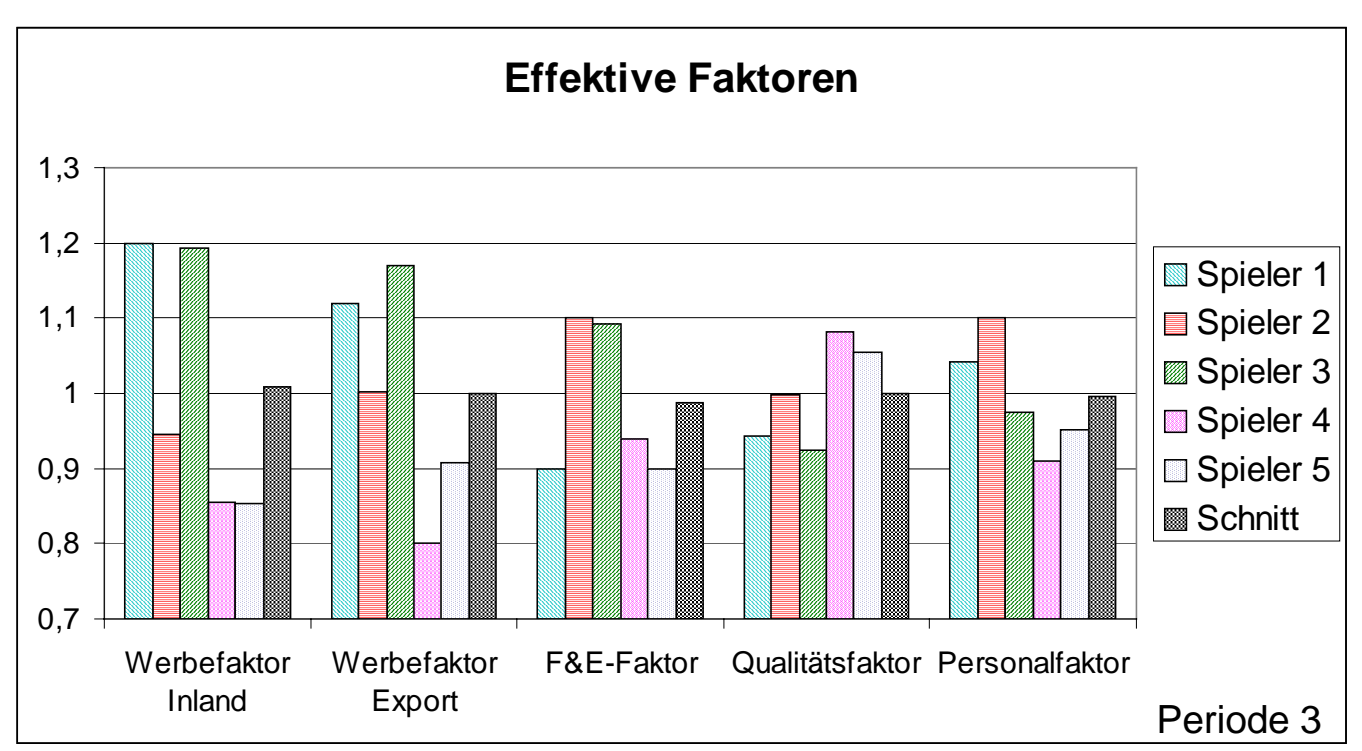

Abb. 76: Spielleiterauswertung Effektive Faktoren

Analysiert man z.B. den Werbefaktor Inland so erkennt man, dass die effektiven Werbeausgaben der Spieler sehr unterschiedlich sind. Spieler 1 und 3 investieren im Verhältnis zu den drei restlichen Unternehmen sehr viel in Werbung. In diesem Fall wird die definierte Begrenzung von maximal 1,2 erreicht (siehe Kapitel 4.3.1.3) Der Spielleiter (aber auch der Spieler) hat die Möglichkeit, mittels einer Detailanalyse festzustellen, um wie viel der effektive Werbeaufwand zu hoch ist. Je nach Situation kann die Spielleitung dem Spieler dies mittels einer E-Mail mitteilen.

\subsection{Das Computerprogramm für den Spielleiter}

Das Spielleitermodul wird als eigenständiger Programmcode zur Verfügung gestellt. Es ist wie auch das Spielermodul in Visual Basic for Applications unter Microsoft Excel 97 in Windows 95 - bzw. Windows NT - Umgebung programmiert. 
Im Verzeichnis SPIELLEITER befindet sich folgende Dateien und Ordner:

\section{Dateien}

1. HANDBUCH Die als HTML-formatierte Datei startet automatisch das Handbuch.

2. LIESMICH Hier werden Informationen zur Installation beschrieben.

3. START Das geschützte Startmodul.

4. LPLUSMOD Das geschützte Spielerleitermodul.

\section{Verzeichnisse}

1. DATA Hier werden (in weiteren Unterverzeichnissen) die Daten der Spieler gespeichert.

2. GRAPHICS Speicherort für automatisch erstellte Graphiken beim Einlesen bzw. Versenden der Ergebnisse einer Periode.

3. INFOS Berichte der Spielleitung. Diese können direkt aus dem Spielleitermodul aufgerufen werden.

4. MANUAL Hier liegen die Daten des (Spieler-) Handbuches.

5. SPIELER Damit die Spielleitung die Möglichkeit hat, direkten Einfluss auf die Entscheidungen der Spieler zu nehmen, befindet sich hier eine (abgeänderte) Version des Spielermoduls. 


\section{Logistikausbildung mit LogisticPLUS}

Training is ,systematic and scheduled, not a
rescue effort summoned to solve the
problem of the moment. In other words,
training should be a process, not an
event." 192

Andrew S. Grove

\subsection{Zielgruppenidentifikation}

Unternehmensspiele dienen heute als wertvolle (ergänzende) Instrumente in der Managementausbildung. ${ }^{193}$ Aufgrund der ursprünglichen Fokussierung auf logistische Fragestellungen ist die Hauptzielgruppe des vorliegenden Planspiels LogisticPLUS, Logistikmanager aus- und weiterzubilden. Wie in den vorangegangenen Kapiteln verdeutlicht, sind für die erfolgreiche Arbeit als Logistiker bestimmte Fähigkeiten und Kenntnisse erforderlich. Neben dem fachlichen (Logistik-) Wissen müssen insbesondere die Funktionszusammenhänge der unterschiedlichen Unternehmensfunktionen und die ertragswirksamen Zusammenhänge in den Produktionssystemen erkannt werden. Weiterhin muss der Logistiker verstehen und erkennen, wie sich (seine) logistischen Entscheidungen auf den Erfolg der Unternehmung auswirken. Hierdurch kann das Verständnis für betriebliche Probleme in benachbarten Funktionsbereichen gestärkt sowie ein Abbau des Ressortdenkens erreicht werden.

Da die Lehre von der industriellen Produktion ein zentraler Teil der Theorie der

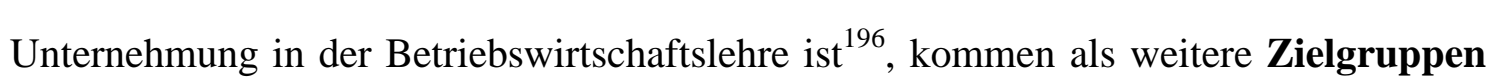
neben Mitarbeitern oder Führungskräften aus dem Bereich der Logistik (insbesondere

\footnotetext{
192 Neff / Citrin (Lessons, 2000) Seite 166. Andrew S. Grove war 1968 Mitgründer und ist seit 1998 Chairman der Intel Corporation.

193 Vgl. etwa Albach (Unternehmensspiele, 1974), Backen et al. (Experimentation, 1992) oder Paich / Sterman (Experimental Markets, 1993).

194 Vgl. Bloech (Unternehmensplanspiele, 1999) Seite 420.

195 Puma (Spielanleitung, 1992) Seite 11.

196 Bloech (Produktionsplanung, 1999) Seite 408.
} 
Produktions-) Manager auf allen Ebenen, Nachwuchsführungskräfte ${ }^{197}$ und

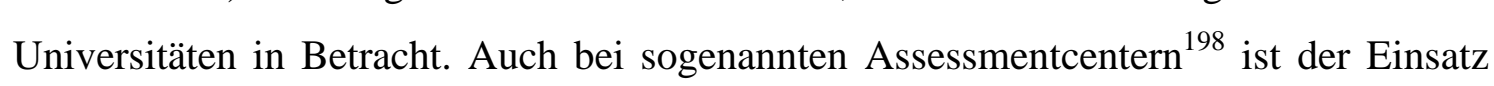
von Planspielen durchaus sinnvoll.

Für den ersten Kontakt mit Planspielen bieten sich Universitäten an. ${ }^{200}$ Hier werden eine große Anzahl von Planspielen neben der wissenschaftlichen Forschung insbesondere zur Ausbildung der Studenten in den Studiengängen Betriebswirtschaft, Wirtschaftsinformatik oder Wirtschaftsingenieurwesen eingesetzt. Die beobachteten Lernerfolge sind durchwegs positiv.

\subsection{Einsatzmöglichkeiten}

Das Planspiel LogisticPLUS kann auf Grund seines von Unternehmen und Branchen unabhängigen Aufbaus vielen Anforderungen gerecht werden:

1. Es sind die (grundsätzlichen) Unternehmensfunktionen Produktion, Logistik, Absatz und (Finanz-) Berichtswesen vorhanden. Wie im Kapitel 3 unter „Arten und Einteilung von Planspielen“ dargestellt, ist ein genereller Umfang mit den sich ergebenden Vorteilen gegeben: Generelle Planspiele betonen betriebswirtschaftliche Zusammenhänge und Interdependenzen sowohl zwischen den unternehmerischen Teilbereichen als auch zwischen einem Unternehmen und den Beschaffungs- und Absatzmärkten.

2. Die Fertigungsstruktur ist einerseits überschaubar, andererseits so komplex aufgebaut, dass verschiedenste Ausgangssituationen geübt werden können. Weiterhin ist durch die gewählte mehrstufige Mehrproduktartenfertigung eine

197 Z.B. Trainee's.

198 Ein Assessment Center ist eine Kombination verschiedener Verhaltens- und Arbeitsproben und kann sich etwa über einen halben bis zu mehreren Tagen erstrecken. Ursprünglich wurden Assessment Center bei der Auswahl des Offiziernachwuchses eingesetzt. Vgl. Hesse / Schrader (Bewerbungsstrategien, 1995) Seite $217 \mathrm{ff}$.

199 Man könnte sich vorstellen, dass ein oder mehrere Bewerber innerhalb kurzer Zeit einerseits eine komplexe Umgebung analysieren und auf Grund einer vorgegebenen Strategie eine begründete Entscheidung fällen müssen. Die Auswirkungen der Entscheidungen sind sofort messbar und können einen weiteren Input für die Bewertung der Bewerber geben.

200 Zum Planspieleinsatz an deutschen Hochschulen vgl. Bronner / Kollmannsperger (Planspieleinsatz, 1997).

201 Vgl. hierzu z.B. Bloech et al. (Ausbildung, 1996), Drössler (Planspiele, 1998), Heidenberger et al. (F\&EUnternehmensspiel, 2001) oder Knüwer (Hochschulen, 1998). 
Vielzahl von Produktionsstrukturen aus der Praxis abgedeckt. Letztendlich wird durch die Fertigungsstruktur gewährleistet, dass ein einzelner Spieler große Schwierigkeiten bei der Bewertung der einzelnen Entscheidungen haben wird. Somit ergibt sich der notwendige Zwang nach einer Spielergruppe bzw. nach Teamarbeit.

3. Es liegt ein oligopolistischer Wettbewerb vor. Der Erfolg einer Firma hängt auch von den Entscheidungen der Konkurrenten ab: damit wird garantiert, dass die eigenen (Plan-) Entscheidungen und daraus abgeleitete (Plan-) Bilanzen in der Tat Planwerte und keine von Anfang an festliegende Ergebnisse sind.

4. Aufgrund der Wahl des Mediums Computer und insbesondere des Datenaustausches über E-Mail ist das Spiel mit regional verteilten Gruppen möglich. Hierbei ist auch der internationale Einsatz mit ggf. unterschiedlichen (Bedienungs-) Sprachen zu nennen.

5. Der Spielleiter hat die Möglichkeit, nach bzw. vor jeder Periode auf das Marktgeschehen Einfluss zu nehmen und Konjunkturverläufe und spezifische Situationen einzustellen. Damit können z.B. „Unvorhergesehenheiten“ wie Lagerbrand oder LKW-Fahrer-Streik oder auch regulative Entscheidungen wie Zuweisung eines Sonderabsatzvolumens aktiv eingesteuert werden.

Nachfolgend seien drei Ausgangslösungen und damit unterschiedliche Herausforderungen für die Spieler bzw. den Logistiker kurz angerissen: 203

1. Marktbehauptung und klassischer Wettbewerb. Hier starten alle fünf Konkurrenten mit den gleichen Bedingungen. Für jedes Unternehmen gilt die gleiche Zielsetzung, wie beispielsweise maximalen Gewinn zu erwirtschaften oder einen bestimmten Marktanteil zu erreichen.

2. Markteintritt im Sinne von Existenzgründung: Vier Unternehmen teilen sich den Markt, ein Unternehmen startet mit wenig Kapital, wenig Produktion (und damit wenig Produktionsmitteln) aber guter Kostenstruktur. Ziel für das Unternehmen ist

202 Gemeint ist hiermit der Zustand der einzelnen Unternehmungen zum Anfang des Spiels, also in der nullten Periode.

203 Prehm verweist auf zwei grundsätzliche Typen von Ausgangssituationen: (a) Die Teilnehmer übernehmen einen laufenden Geschäftsbetrieb, in dem Anlagen, Rohstoffe, Fertigerzeugnisse, Vertriebseinrichtungen, etc. bereits vorhanden sind oder (b) sie gründen ein Unternehmen ausschließlich mit Hilfe der bereitgestellten Finanzmittel. Die Ausgangslage muss nicht notwendigerweise für alle Spielgruppen gleich sein. Vgl. Prehm (Marketingplanspiel, 1995) Seite 19. 
es, den etablierten Unternehmen aufgrund der bestehenden Kostenvorteile Marktanteile abzunehmen.

3. Markteintritt im Sinne von Behauptung gegen starke Konkurrenz: Hier ist ein Unternehmen dominanter Marktführer (Marktanteil z.B. 60 \%), die vier restlichen Unternehmen starten mit einem Marktanteil von jeweils $10 \%$. Die Aufgabe für die vier Unternehmungen könnte lauten erstens die drei direkten Wettbewerber zu überflügeln und gleichzeitig die Position des Marksführers zu schwächen.

Um möglichst reale Bedingungen zu schaffen, hat die Spielleitung die Möglichkeit, Eingriffe in das aktuelle Spielgeschehen vorzunehmen. So können Lern- und Erfahrungskurven in Form von Produktivitätsverbesserungen (und damit Kostensenkungen), Saison- und Konjunkturschwankungen in Form von starker Nachfrageänderung, politische Einflüsse in Form von nicht zugestellten Lieferungen oder bei der Änderung der Steuerlast oder auch direkte Markteinflüsse simuliert werden.

Diese flexible Anpassbarkeit von LogisticPLUS erlaubt damit den Einsatz in unterschiedlichsten Zielgruppen. Je nach Lernziel und Voraussetzungen der Teilnehmer ist eine Anpassung an die jeweiligen Erfordernisse möglich, ohne die Teilnehmer zu überfordern oder sie bewusst herauszufordern. Diese Anpassungen sind leicht implementierbar, es müssen nur einige Variablen wie z.B. Bestände geändert werden. 204

LogisticPLUS ist aufgrund seines Aufbaus in verschiedenen Arten spielbar:

1. Seminar: Hier befinden sich die Spieler für beispielsweise drei Tage in der gleichen lokalen Umgebung. Geeignet ist ein Seminarraum mit großen Tischen und geeigneter Multimediaausrüstung 205 . Der Spielleiter hat so die Möglichkeit, Fragen direkt zu beantworten bzw. zusätzliche Lehrmodule einzubringen.

2. Fernplanspiel: Die Spielerteams befinden sich an unterschiedlichen Orten. Sie treffen ihre Entscheidungen und verschicken die Daten z.B. per E-Mail an die Spielleitung. Das hat den Vorteil, dass keine feste Terminplanung im Sinne eines zusammenhängenden Zeitraumes notwendig ist. Die Teilnehmer können sich auf bestimmte Abgabetermine wie z.B. einen Wochentag einigen. So kann jedes Team

204 Im Planspiel sind drei stringente Sätze von Startvariablen als feste Module installiert. 
eigenständig und unabhängig von den Mitwettbewerbern seine Planungen erstellen. Weiterhin besteht die Möglichkeit, unterschiedliche Sprachoberflächen zu benutzen. Diese gestattet es, dass z.B. Teams aus unterschiedlichen Kontinenten in deren jeweiliger Landessprache planen können. Die dann vermutlich englischsprachigen Auswertungen der Spielleitung sollten in diesem Fall jedoch von allen Teilnehmern verstanden werden. Andererseits haben die Spieler keinen direkten Kontakt zum Spielleiter. Die Unterlagen wie z.B. das Handbuch sind dementsprechend ausführlich zu gestalten.

3. Assessmentcenter: Hier haben Unternehmen die Möglichkeit, Stellenbewerber in verschiedenen Bereiche zu analysieren. So gilt es zu prüfen wie und auf welcher Informationsbasis ein Einzelner eine Entscheidung trifft oder welche Stellung eine Person in einem Team einnimmt. Weiterhin kann bei der Reduktion des Planspiels auf eine Periode und bei fester Voreinstellung der vier Wettbewerber die Qualität der Entscheidung objektiv gemessen werden.

Für alle drei Arten der Planspieldurchführung können verschiedene gemeinsame gruppenbezogene Ausbildungsziele erreicht werden:206

- Die Teilnehmer werden gezwungen, Gruppen- oder Teamarbeit zu praktizieren.

- Voraussetzung für eine gute Gruppenzusammenarbeit ist die Kommunikation in der Gruppe; die Kontaktfähigkeit der Teilnehmer wird verbessert.

- Die willkürliche Zusammensetzung der Gruppe verlangt das Auffinden einer effektiven Organisationsform.

- Eine effektive Gruppenarbeit verlangt von jedem Teilnehmer eine aktive Mitarbeit am Entscheidungsprozess. Der Spieler muss Durchsetzungsvermögen beweisen, aber auch die Fähigkeit zur Selbstkritik besitzen.

- Die Koordination von Plänen und Entscheidungen wird geübt.

\footnotetext{
205 Fünf Spielcomputer, ein Computer für den Spielleiter, Flipchart, ggf. Beamer und Overhead-Projektor.

206 Puma (Spielanleitung, 1992) Seite 12.
} 


\subsection{Seminarkonzeption und Lehrmodule}

Planspiele und Unternehmenssimulationen sind keine Selbstläufer, sondern sie bedürfen der Einbettung in ein Seminarkonzept oder noch besser in ein Lernkonzept, das über das eigentliche Seminar hinausreicht. Die Seminargestaltung ist - neben der Modellkonzeption - ausschlaggebend für die Lerneffektivität. Hierbei sind insbesondere auch die Aspekte zu betrachten, die dem eigentlichen Ablauf von Unternehmensspielen vor- oder nachgelagert sind und erheblich zum Erfolg von Unternehmenssimulationen beitragen.

Unterscheidet man den Einstieg und Verlauf von Planspielen zeitlich, so kann man in drei Phasen unterteilen:

1. den Seminareinstieg durch die Kombination der Unternehmenssimulation mit anderen Lehrmethoden,

2. den Seminarablauf unter besonderer Berücksichtigung der Reflexion von Lerninhalten und

3. das Lernen nach dem Seminar unter Berücksichtigung des Lernkonzeptes.

\subsubsection{Seminareinstieg}

In der ersten Phase stellt sich häufig das Problem, dass die Vielzahl von Informationen die Spieler überfordern. Das Hineinfinden in die komplexe Materie ist bei einem Neueinstieg für viele Teilnehmer schwer. So lassen sich die zur Verfügung gestellten oft sehr detaillierten Berichte oder Beschreibungen aufgrund der ungewöhnlichen Darstellung oder aufgrund mangelhafter Transparenz nur schwierig interpretieren. Weiterhin liegen keine direkten Informationen über die Wirkungen der einzelnen Entscheidungsparameter vor.

Vor dem Beginn und der Durchführung des Planspieles muss sich die Spielleitung grundlegende Fragen stellen:

\footnotetext{
207 Vgl. Achtenhagen (Lernhandeln, 1992) Seite 182 ff. oder Schöl (Modellentwurf, 1992) Seite 196.

208 Prehm (Marketingplanspiel, 1995) Seite 19.
} 
1. Welche Ausbildungsziele sollen durch den Einsatz des Planspiels erreicht werden? 210

2. Welche Qualifikationen weisen die Teilnehmer auf und - sofern es sich um Teilnehmer aus der Praxis handelt - wie sind sie hierarchisch im Unternehmen eingeordnet?

3. Wie viel Zeit steht für das Seminar zur Verfügung ?

4. Auf welche Hilfsmittel (z.B. Taschenrechner) können die Teilnehmer während des Seminars zurückgreifen ?

Bei der Definition des Planspiels und in der Ausführung der Computerprogramme wurde versucht, diese Probleme zu berücksichtigen. So besteht z.B. die Möglichkeit, die Komplexität durch die Beschränkung auf nur einen (Inlands-) Markt oder durch automatische Bestellung der Transportkapazitäten zu reduzieren. Weiterhin ist das als HTML-Dokument vorliegende Handbuch als eigenes Computerprogramm konzipiert, so dass es parallel zum Planspiel als Nachschlagewerk zu verwenden ist.

Kenntnisse der Anleitung für das Planspiel sowie Einblicke in verschiedene betriebswirtschaftliche Bereiche sind notwendige Kriterien für die erfolgreiche Teilnahme und damit für die Erzielung eines Lernerfolges. Für den Zeitpunkt der Übergabe der Spielanleitung an die Spieler gibt es unterschiedliche Meinungen: Rohn hält die rechtzeitige Zusendung der Spielunterlagen an die Teilnehmer vor Beginn des Spiels für unerlässlich, damit diese sich mit der Aufgabenstellung vertraut machen können. Dagegen rät Ceppi von dieser Vorgehensweise mit der Begründung ab, dass in der Regel kaum mehr als die Hälfte der Teilnehmer die Arbeitsunterlagen ausreichend bearbeiten, um auf eine Kurzeinführung zu Beginn des Seminars genügend vorbereitet zu sein.

Ohne die Grundlagen der Betriebswirtschaftslehre - hier sind besonders die Bereiche Logistik, Produktion, Absatz und (internes) Rechnungswesen zu nennen - und ohne das

209 Schöl (Modellentwurf, 1992) Seite 196.

210 Vgl. hierzu die Ausführungen im Kapitel 3.

211 Weitere Informationen im Internet unter http://www.LogisticPLUS.de.

212 Vgl. Rohn (Unternehmensplanspiel, 1964) Seite 87, Ceppi (Management Games, 1970) Seite 307 oder auch Prehm (Marketingplanspiel, 1995) Seite 20, der die These von Ceppi durch eigene Erfahrungen unterstützt. 
Verständnis für die funktionellen Zusammenhänge der einzelnen (Modell-) Funktionen sind die Entscheidungen unter dem Gesichtspunkt der Optimierung jedoch nicht zu lösen. 213

Nachfolgend ein Vorschlag für betriebswirtschaftliche Lehrmodule, die während des Spiels entweder im Selbststudium, als Vortrag oder durch gemeinsames Üben zu erlernen wären. ${ }^{214}$ Die detaillierte Ausführung bzw. die Inhalte der Modelle werden sich mit vermehrtem Einsatz des Planspiels ergeben.

\begin{tabular}{|l|l|}
\hline Thema & Inhalte \\
\hline $\begin{array}{l}\text { Grundfunktionen } \\
\text { der Logistik }\end{array}$ & $\begin{array}{l}\text { Darstellung der logistischen Zusammenhänge in Spiel und Betrieben. } \\
\text { Zusammenhänge zwischen Erfolg und Logistik. } \\
\text { Strategische und operative Logistikplanung. Kennziffern und ihre Aussagen. }\end{array}$ \\
\hline Produktion & $\begin{array}{l}\text { Produktion, Qualitätsmanagement und Produktionslogistik. } \\
\text { Aufgaben und Ansätze der Produktionslogistik. }\end{array}$ \\
\hline Preis- / Absatztheorie & $\begin{array}{l}\text { Preisbildung, Preisfunktionen und Nachfrage. } \\
\text { Umsatzfunktion, Deckungsbeitragsrechnung und Gewinnfunktion. }\end{array}$ \\
\hline Erfolgsdarstellungen & $\begin{array}{l}\text { Bilanz, GuV, Vollkostenrechnung, Deckungsbeitragsrechnung, Cashflow- } \\
\text { Rechnung, Liquiditätsrestriktionen. }\end{array}$ \\
\hline Kapitalgesellschaften & Gesellschaftsformen, Steuerrecht, Bilanzierungspflichten. \\
\hline Investitionen & Tatbestände der Investitionsvorhaben. Bedeutung und Merkmale. \\
\hline Strategische Planung & $\begin{array}{l}\text { Strategische Potenziale, Umweltanalyse, Unternehmensanalyse. } \\
\text { Portfolios, Strategien, Strategieumsetzung. }\end{array}$ \\
\hline
\end{tabular}

Tab. 44: Lehrmodule und Inhalte

Eine Möglichkeit der Wissensvermittlung der obigen Lehrmodule wäre die Schulung der Teilnehmer durch die Teilnehmer selbst. So könnten die obigen Module so aufgeteilt werden, dass jeder Teilnehmer sich vor dem eigentlichen Planspiel in ein Thema vertiefend einarbeitet und das erworbene Wissen in einem Kurzvortrag allen Mitspieler zugänglich macht.

Ein weiterer wichtiger Punkt ist eine detaillierte Zeitplanung durch die Spielleitung, insbesondere für die Durchführung als Blockveranstaltung. Hierbei ist zwischen der theoretischen Wissensvermittlung, einer Eingansbesprechung, dem Erlernen der

213 Pack weist darauf hin, dass es z.B. nicht sinnvoll ist, „Planspiele als alleinige Lehrmethode für die Aus- und Weiterbildung zu verwenden. Die meisten Spiele setzen durch Schulung oder praktische Erfahrung erworbenes Wissen voraus; ein Wissen also, dass vorher mittels anderer Lehrmethoden den Spielern vermittelt worden sein muss." Pack (Planspiele, 1968) Seite 14.

${ }^{214}$ Hierbei spielt die Form der Planspieldurchführung (z.B. als Fernplanspiel, im Rahmen einer wöchentlichen Seminars oder als Blockveranstaltung) eine wichtige Rolle. 
Bedienung des Computerprogramms, der gruppeninternen Analyse des aktuellen Spielstandes und der Entscheidungsfindung, der Auswertung durch die Spielleitung sowie einer Abschlussbesprechung $\mathrm{zu}$ unterscheiden. Je komplexer ein Unternehmensplanspiel gestaltet ist und je stärker die Teilnehmer zur gründlichen Planung ihrer Entscheidungen angehalten werden sollen, desto größer wird der Bedarf an Entscheidungszeit. 215

\subsubsection{Seminarablauf}

Es ist die erste Aufgabe eines jeden Teams, die Spielanleitung bzw. das Handbuch zu lesen. Jeder im Team muss über Strukturen, Produkte, Absatzwege und Bedienung Bescheid wissen. Eine Einführungsveranstaltung kann die Teilnehmer über die Zusammenhänge informieren.

Danach sollten die idealer weise aus drei Spielern gebildeten Teams sich nach Verantwortungsbereichen aufteilen. Möglich ist beispielsweise die im Kapitel 4 vorgeschlagene Aufteilung in Produktion, Vertrieb und Service. Ischebeck weißt in diesem Zusammenhang darauf hin, dass der Lerneffekt durch eine eindeutige Kompetenzübertragung jedes Teilnehmers gesteigert werden kann, da dieser einerseits die ihm übertragenen Planungsaufgaben und Entscheidungen - in Abstimmung mit den anderen Mitgliedern - selbständig durchführt und andererseits die daraus resultierenden Ergebnisse gegenüber seinen Mitspielern vertreten muss. 216 Jeder der Spieler sollte sich dabei einen Bereich suchen, der ihm aufgrund seiner bisherigen Kenntnisse eher fremd ist. Dadurch besteht die Möglichkeit, neue Problemebereiche kennen zu lernen und zu verstehen und somit ein größeres Verständnis für das Gesamtunternehmen zu erlangen. 217

Ein gutes und von LogisticPLUS unterstütztes Verfahren ist das Durchführen einer Proberunde. Hier besteht die Möglichkeit Missverständnisse bei den Teilnehmern zu

\footnotetext{
215 Prehm (Marketingplanspiel, 1995) Seite 21.

216 Ischebeck (Unternehmensspiele, 1969) Seite 602.

217 Vgl. Prehm (Marketingplanspiel, 1995) Seite 24.
} 
erkennen sowie zu verhindern, dass Fehlinterpretationen gravierende Fehler in den Folgeperioden nach sich ziehen. Die Gruppenmitglieder können selbst prüfen, ob sie die Ausgangssituation verstanden bzw. richtig eingeschätzt haben und darauf aufbauend richtige Entscheidungen getroffen haben. Weiterhin haben die Spieler die Möglichkeit, erste Erkenntnisse über das Reaktions- bzw. Marktmodell zu erlangen.

Die Seminarphase selbst birgt häufig das Problem, dass lediglich die Beherrschung des Simulationsmodells angestrebt wird, jedoch kein Wissen für die betriebliche Realität hinzugewonnen wird. ${ }^{219}$ Daher ist es nötig, dass die Spielleitung regelmäßig Auswertungen, Hilfestellungen und Hinweise an die Spieler gibt und damit Reflexionen der Lerninhalte aktiviert. Diese im englischen als debriefing bezeichnete Reflexion dient dazu, die Teilnehmer zum Nachdenken über ihre Lernerfahrungen anzuregen und ihnen zum Verständnis der Anwendung bzw. Anwendungsmöglichkeiten ihres Wissens in der betrieblichen Praxis zu verhelfen.

LogisticPLUS erlaubt es jedem Team, Auswirkungen und Ergebnisse der Planung vor der eigentlichen Marktsimulation unmittelbar zu sehen. Preise und geplante Absatzmengen als Zielgrößen haben direkten und sofortigen Einfluss auf die Finanzund Betriebskennzahlen. Jedes Team kann z.B. im eigenen Spielermodul prüfen, ob

1. genügend Rohstoffe für die geplante Produktion (genügend Halbfabrikate für die Montage) vorhanden sind,

2. die Lager-, Maschinen- oder Personalkapazitäten ausreichen (oder ob teure Überkapazitäten bereitgestellt werden müssen),

3. genügend LKW bereitgestellt wurden,

4. ob die Einzahlungen für die geplanten Investitionen und die laufenden Ausgaben wie Löhne oder Produktionsmittel ausreichen,

5. bestimmte Lager den notwendigen Lagerumschlag aufweisen,

6. $\quad \ldots .$. 
Als Berichtsformen stehen in der Planungsphase alle in Kapitel 4 beschriebenen Funktionen bis auf die graphischen Auswertunger 220 zur Verfügung. Beim Verlassen des Moduls „Entscheidungen“ und dem direkten Wechsel in die Menüs „Rechnungswesen“ oder „Betriebskennzahlen“ werden alle funktionalen Zusammenhänge des Planspiels realitätsnahe ausgeführt. ${ }^{221}$ Die finanziellen Instrumente wie GuV, Bilanz, Cashflow, Liquidität und Kostenrechnung werden komplett berechnet. In der Planphase ist allerdings nicht berücksichtigt, welche Entscheidungen durch die vier Konkurrenten getroffen werden. Da diese insbesondere bei der Bestimmung des Durchschnittspreises und damit des eigenen Absatzes einen sehr großen Einfluss haben, ist die Aussagefähigkeit dieser Instrumente - wie in der Realität deutlich sichtbar naturgemäß begrenzt. Gerade hiermit wird ein wertvoller Beitrag zum gegenseitigen Verständnis von Theoretikern und Praktikern geleistet: Beide können erkennen, dass (theoretische) Modelle eine gute Grundlage für eine Entscheidungsfindung sind, dass aber die in der Praxis vorkommenden Einflussfunktionen - wie z.B. Preisnachlassentscheidungen der Konkurrenz - nur sehr schwer modellierbar und damit für den Einzelfall nicht vorhersehbar sind.

Zur Abrundung der Durchführung des Seminars ist nach der letzten Runde eine Abschlussbesprechung mit allen Teilnehmern des Planspiels sinnvoll. Zuerst sollten kurz nochmals die Ziele von Planspielen und das Ziel der Veranstaltung angesprochen werden. Nachfolgend ist eine kurze Präsentation jeder Gruppe vorzusehen. Hier haben die Gruppen die Möglichkeit, rückblickend ihre Unternehmensziele sowie die geplanten strategischen und taktisch / operativen Maßnahmen im Zeitablauf darzustellen und selbstkritisch aufzuzeigen, inwieweit die Ziele nicht oder nur teilweise erreicht wurden. Darüber hinaus erläutern die Teilnehmer ihre Teamorganisation sowie deren Stärken und Schwächen. Im Anschluss daran präsentiert die Spielleitung die finalen Ergebnisse in Form einer detaillierten Auswertung, die einerseits alle Unternehmen sowie jedes einzelnen Unternehmen beinhaltet. 222

\footnotetext{
${ }^{220}$ Gemeint sind die EXCEL-Auswertungen der aktuellen, dann abgeschlossenen Periode, die mit Beginn der neuen Periode im Verzeichnis \GRAPHICS \zur Verfügung stehen.

221 Damit entfällt die in vielen Planspielen nötige Überprüfung der Daten auf formale Richtigkeit. Vgl. hierzu Prehm (Marketingplanspiel, 1995) Seite 25.

222 Vgl. hierzu Prehm (Marketingplanspiel, 1995) Seite 28.
} 
Die Spielauswertung ist eine vom Umfang her große Reflexion, die weitergehende Punkte wie Ergebnisse, Zielerreichung, Seminarkritik oder Evaluation beinhaltet.

\subsubsection{Nachbereitung des Seminars}

Nach der Durchführung Planspiels gibt insbesondere für die Spielleitung die Aufgabe, die Veranstaltung nachzubereiten. Hierbei stehen zwei Aspekte im Vordergrund. 223

1. Konnten die gesetzten Ausbildungsziele aus Sicht des Veranstalters - z.B. des Unternehmens oder des Lehrstuhlinhabers - erreicht werden ?

2. Welche Verbesserungen sollten an dem Unternehmensspiel und am Ablauf der Veranstaltung bei zukünftigen Seminaren vorgenommen werden?

Zusammenfassend kann festgestellt werden, dass Reflexionen und Auswertungen stark zum Verständnis und damit zum Lernerfolg beitragen. Die Aufbereitung der Ergebnisse - bei den Reflexionen als Marktforschungen, bei der finalen Auswertung automatisch ähnelt einem Betriebsvergleich oder Benchmark. Diese haben unmittelbare Bezüge zur Praxis, da insbesondere mittelständische Unternehmen den Betriebsvergleich als wichtiges Analyseinstrument nutzen. 224

223 Prehm (Marketingplanspiel, 1995) Seite 30.

224 Vgl. Schöl (Modellentwurf, 1992) Seite 202. 


\section{Zusammenfassung}

Ein erfolgreicher Unternehmer wird von einem Journalisten gefragt, worauf er seine Erfolge zurückführe. Antwort: „Richtige Entscheidungen“. Der Journalist fragt weiter: „Entschuldigen Sie, wie kommen Sie denn $\mathrm{zu}$ Ihren richtigen Entscheidungen?“ Der Unternehmer: „Erfahrung.“ Der Journalist, allmählich verzweifelt: „Aber wie kommen Sie denn zu Ihrer Erfahrung?“ Der Unternehmer: „Falsche Entscheidungen.

Hans Georg Plaut

LogisticPLUS ist ein Planspiel mit dem Schwerpunkt auf Produktions- und Logistikfragestellungen. Bei der Definition der Unternehmensfunktionen sowie bei der Ausführung der Programmmodule wurde auf die Unabhängigkeit von bestimmten Verfahren, Sprachen oder Standorten Wert gelegt. Geringe Erweiterungen oder Änderungen ermöglichen es, Teams aus verschiedenen Kontinenten gegeneinander antreten zu lassen. Dabei könnte z.B. jedes Spielermodul in unterschiedlichen Sprachen ausgeführt werden.

Die anfangs getroffene Entscheidung für die Programmiersprache Visual Basic for Applications von Microsoft stellt sich auch im Nachhinein als richtig heraus. Zum einen ist die Sprache modular aufgebaut. Dies ermöglicht sowohl eine moderne Programmierung als auch unkomplizierte Änderungen (z.B. im Steuerrecht) oder Ergänzungen (neue Finanzkennzahlen wie EBIT ${ }^{226}$ oder EVA ${ }^{227}$ ) selbst seitens

\footnotetext{
Erzählt von Herbert Jacob. Vgl. Anders (Betriebswirtschaft, 1995) Seite 151.

226 EBIT (engl. earnings before interest and taxes): Gewinn vor Zinsen und Steuern.

227 Das EVA-Konzept (EVA, engl. economic value added), begründet durch den US-Amerikaner Bennett Stewart, ist ein sich stark am Marktwert der Aktie orientiertes System zur Beurteilung der Finanzkraft eines Unternehmens. Vgl. Kiesel (Logistik, 1999). Anmerkung: Vereinfacht ausgedrückt ist EVA das Betriebsergebnis eines Unternehmens, vermindert um die Steuern auf Einkommen und Ertrag und vermindert um die Kapitalkosten.
} 
„externer“ Programmierer. Zum zweiten ist die Sprache sehr leistungsfähig und umfangreich. Damit lassen sich auch schwierige funktionale Zusammenhänge kompakt lösen. Drittens liefert die Microsoft-Umgebung und hauptsächlich das Programm ECXEL hilfreiche Tools und Analysemöglichkeiten, auf die mit einfachen Mitteln zurückgegriffen werden kann. Ausdrücklich sei die graphische Auswertung einer Periode erwähnt, die ständig erweitert und angepasst werden kann.

Die Struktur der Unternehmung und damit die zentrale Definition der Abhängigkeiten und Wirkungszusammenhänge erweisen sich ebenfalls als gut anwendbar. Die mehrstufige Verbundproduktion verknüpft mit einem Recyclingprozess weist genügend Interdependenzen auf, die den Spielern genaue Planungen und Kenntnisse der Abhängigkeiten abverlangen. Der simulierte Leistungsfluss ist nicht spezieller, sondern allgemeiner Natur. $\mathrm{Er}$ ist auf verschiedene industrielle Unternehmungen anwendbar, so beispielsweise in der Automobilzuliefer-, Sanitär- und Heizungsindustrie oder für andere Serienfertiger.

Da der Schwerpunkt von LogisticPLUS auf dem technischen Bereich und den damit direkt verknüpften finanziellen Auswirkungen liegt, reicht es aus, zwei reguläre bzw. ständige Absatzmärkte und einen Objekt- bzw. Ausschreibungsmarkt vorzusehen. Auf diese Weise gelingt es, den Entscheidungsaufwand im Absatzbereich auf ein vernünftiges Minimum zu beschränken, andererseits die nötige Unterscheidung in Hoch- und Niederpreismärkte und damit unterschiedliche Deckungsbeiträge sicherzustellen.

Die Definition der Unternehmensfunktionen im Spielermodul erweist sich als sehr praktikabel. Dadurch hat der Spieler unter Ausschluss der Entscheidungen der Wettbewerber die Möglichkeit, sofortige (Plan-) Zahlen über seine Firma zu erhalten. Er kann alle sich direkt oder indirekt ergebenden finanziellen, Stück- und sonstigen Werte einsehen und erste Rückschlüsse auf die Auswirkungen seiner Entscheidungen ziehen. Eine Alternative wäre gewesen, die Unternehmensfunktionen in das Leitungsmodul zu implementieren. Dadurch hätte sich zwar der Programmumfang der Spielermodule

\footnotetext{
228 Teile dieser Auswertungen sind Abbildungen in den Kapitel 4 und 5.
} 
reduziert, was zu einer leichtern Austauschbarkeit des Programmcodes über das Internet geführt hätte, zugleich aber wäre die gesamte automatische Auswertung in der Planungsphase entfallen.

Die Möglichkeit der aktiven und aktuellen Beeinflussung des Spiels durch die Spielleitung ist sinnvoll und hilfreich. Damit können einerseits generelle Entwicklungen auf den Märkten gesteuert werden, andererseits einzelnen Spielern Unterstützung oder Feedback gegeben werden.

LogisticPLUS verwendet zur Festlegung der Ausgangssituation vor Spielbeginn eine Ausgangslösung. Es besteht damit die Möglichkeit, die Ausgangslösung durch den Spielleiter beliebig zu konfigurieren und zu verändern. Die Ausgangslösung bestimmt weitgehend die notwendigen Entscheidungen, die während der Einsatzphase getroffen werden müssen. Typische Ausgangslösungen sind z.B. Marktbehauptung und klassischer Wettbewerb, Markteintritt im Sinne von Existenzgründung oder Markteintritt im Sinne von Behauptung gegen starke Konkurrenz.

Durch die Möglichkeit des Eingriffs von Seiten des Spielleiters in das aktuelle Spielgeschehen mittels Veränderung der Parameter ist eine weitere Erhöhung der Komplexität erreichbar. Dadurch können insbesondere Lern- und Erfahrungskurven, Saison- und Konjunkturschwankungen, politische Einflüsse, Rationalisierungseffekte und Markteinflüsse simuliert werden.

Darüber hinaus ist es möglich, die Überforderung in der Anfangsphase zu reduzieren, weil beispielsweise nur auf einem Markt gestartet werden kann, die für den Absatz nötigen Transportkapazitäten automatisch bestellt werden oder nur das Produkt Combilus vertrieben wird. Als zusätzliche Alternative in der Einstiegsphase können Test- oder Proberunden gespielt werden, um den Spielern die Auswirkungen ihrer voraussichtlich nicht optimalen Entscheidungen zu demonstrieren.

229 Der Vollständigkeit halber sei darauf hingewiesen, dass beim Datenaustausch nur die Datenfelder und nicht die Programmcodes verschickt werden. Dadurch ist eine möglichst geringe Dateigröße gewährleistet. 
Aufgrund dieser Erkenntnisse und insbesondere der ersten Erfahrungen während der Testphasen kann festgestellt werden, dass - ausgehend von den zentralen Fragestellungen - das Planspiel auf vielfältige und unterschiedliche Einsatzsituationen angewandt werden kann. Es wird augenfällig, dass Logistikmanager durch die Beschäftigung mit dem Planspiel ihr Wissen und Können über die Zusammenhänge der einzelnen Unternehmensfunktionen unter diversen Bedingungen erheblich zu erweitern vermögen.

An der Weiterentwicklung von LogisticPLUS wird gearbeitet. 


\section{Auszug aus dem Programmcode}

Visual Basic for Application ist eine sehr leistungsfähige und dafür relativ einfach zu erlernende Sprache. Durch den modularen und prozeduralen Aufbau ist es möglich, im Nachhinein Erweiterungen oder Ergänzungen einfach und schnell zu verwirklichen.

Um das Gesamtbild der Unternehmenssimulation LogisticPLUS zu vervollständigen werden in diesem Kapitel zwei Auszüge aus dem Programmcode dargestellt. Für den Spieler oder Spielleiter ist die Kenntnis der Sprache nicht notwendig.

Anmerkung: Das einzelne Hochkomma >‘< leitet einen Kommentar ein.

\section{Beispiel 1: Überprüfung der Eingabe auf eine korrekten Wert}

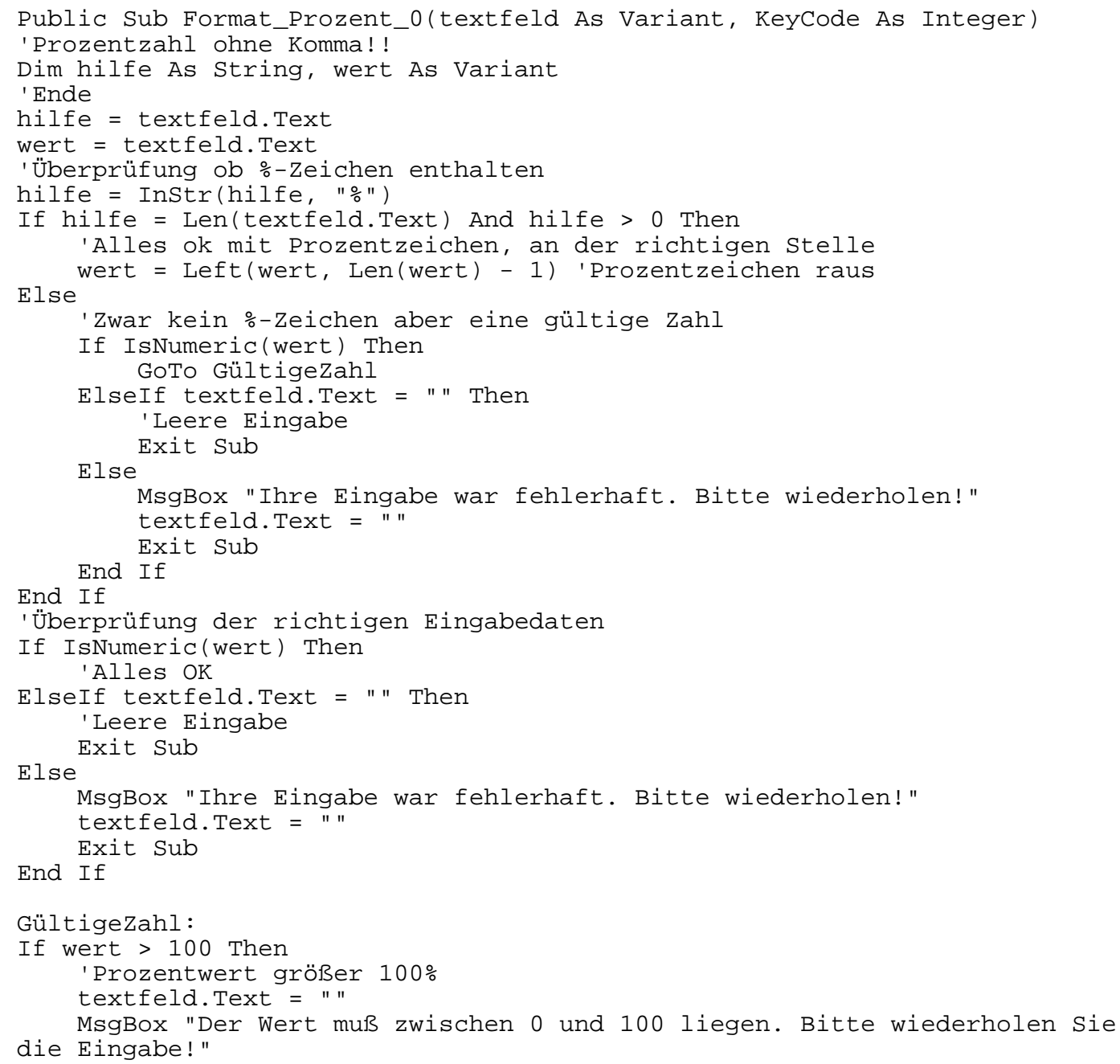




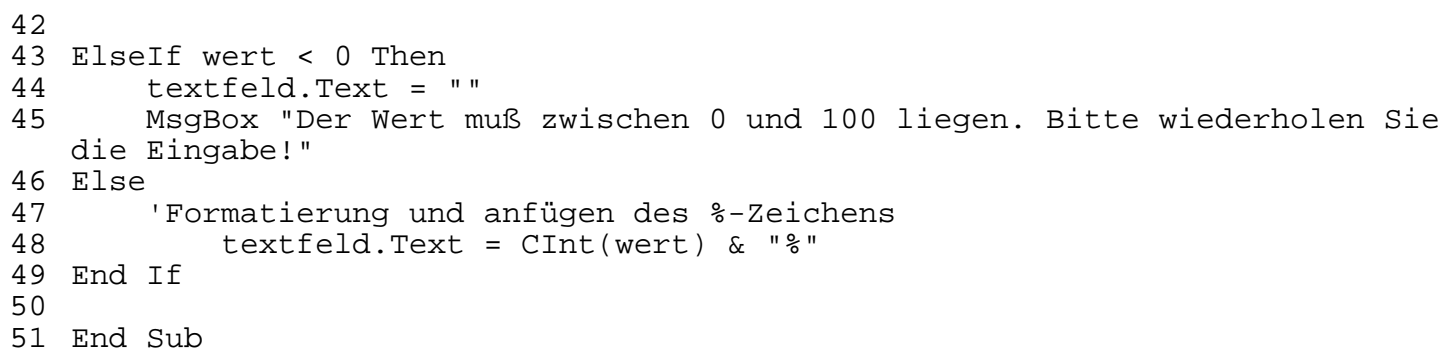

\section{Beispiel 2: Berechnung der maximal möglichen Produktion}

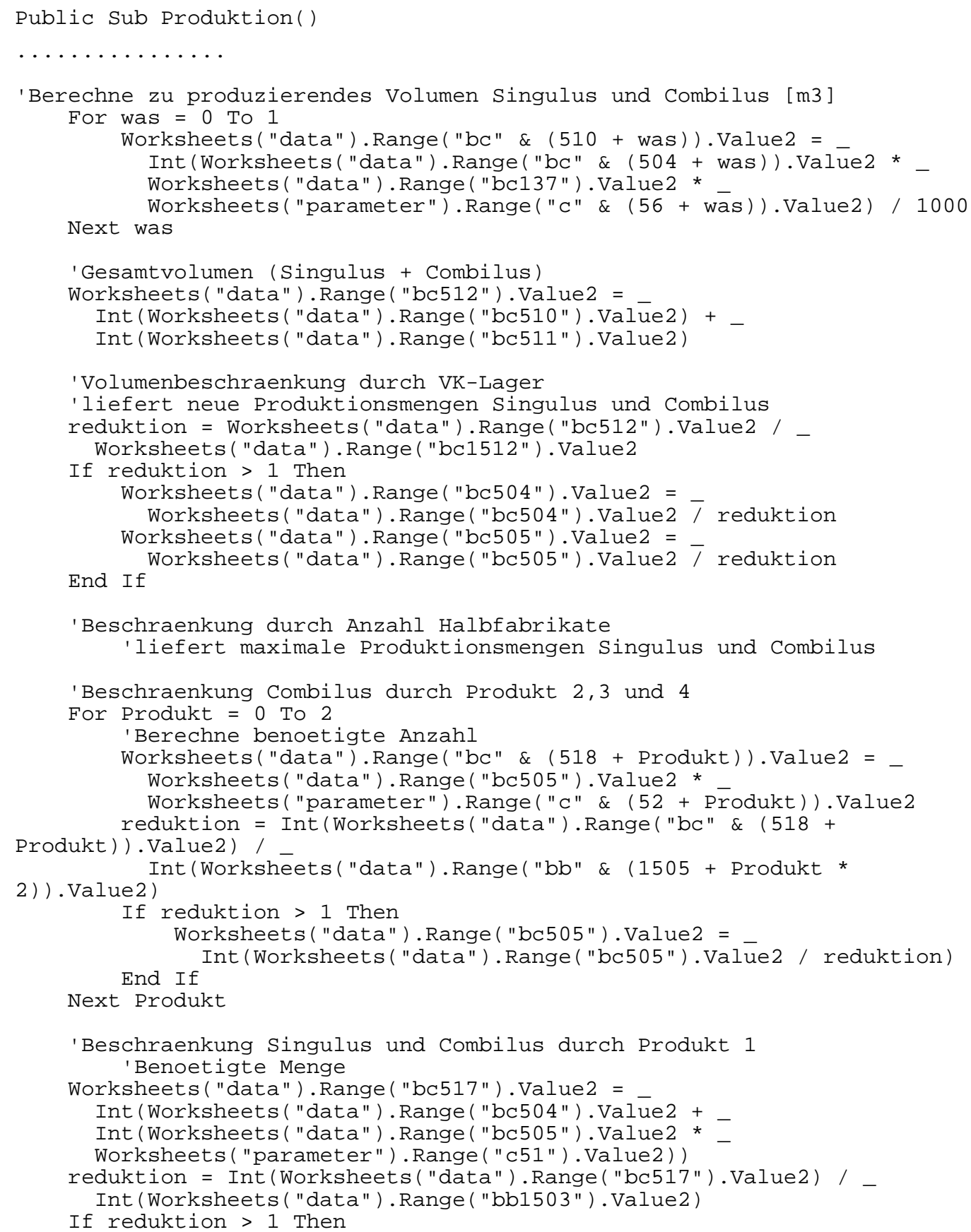




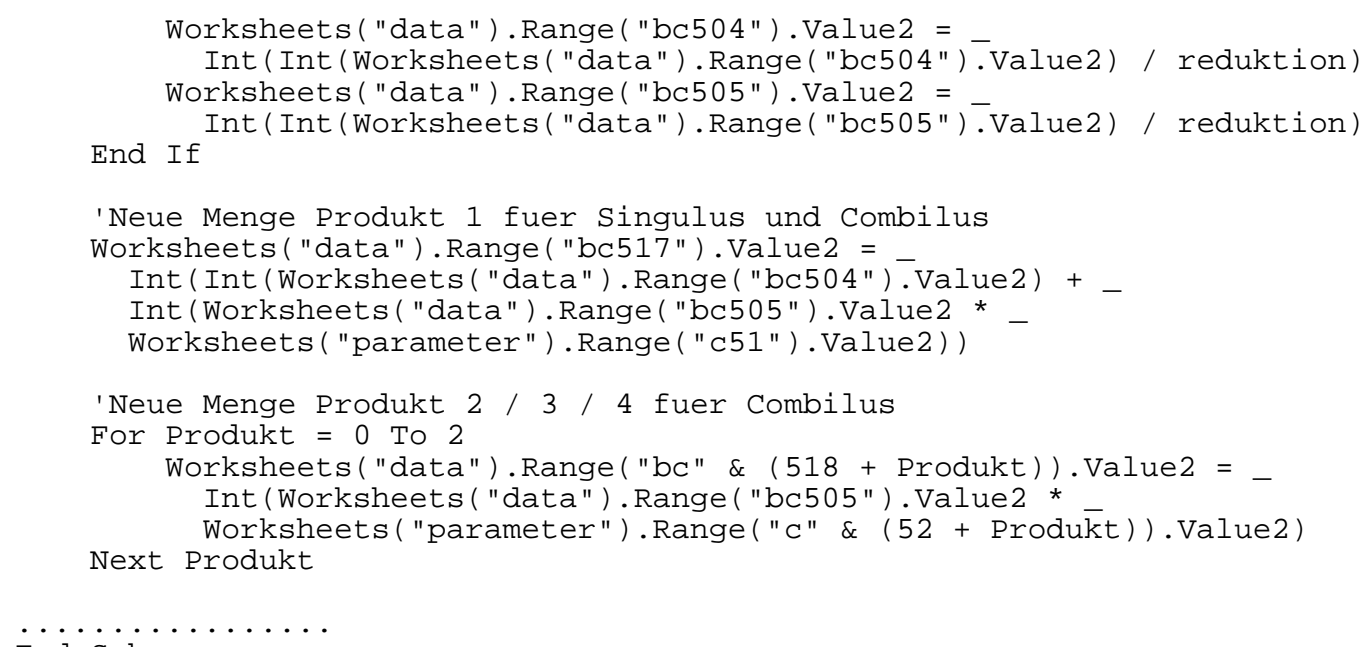




\section{Literaturverzeichnis}

Achtenhagen et al. (Lernhandeln, 1992) - Frank Achtenhagen, Tade Tramm, Peter Preiß, Heiko Seemann-Weymar, Ernst G. John und Axel Schunck: Lernhandeln in komplexen Situationen. Neue Konzepte der betriebswirtschaftlichen Ausbildung. Gabler Verlag, Wiesbaden.

Achtenhagen (Schlüsselqualifikationen, 1997) - Frank Achtenhagen: Schlüsselqualifikationen. In: Eveline Wittman und Jürgen van Buer (Hrsg.): Schlüsselqualifikationen zwischen bildungspolitischem Anspruch, wissenschaftlicher Grundlegung und wissenschaftsadäquater Umsetzung. Studien zur Wirtschafts- und Erwachsenenpädagogik aus der HumboldtUniversität zu Berlin, Band 18.

Albach (Unternehmensspiele, 1974) - Horst Albach: Unternehmensspiele als Mittel der Führungskräfteschulung, In: F. Fasshauer und W. Wurzacher (Hrsg.): Unternehmensspiele - Stand und Entwicklungstendenzen. Stuttgart.

Anders (Betriebswirtschaft, 1995) - Peter E. Anders: Betriebswirtschaft non olet. Gabler Verlag Wiesbaden.

Anderson / Morrice (Simulation Game, 2000) - Edward G. Anderson Jr. und Douglas J. Morrice: A Simulation Game for Teaching Service-Oriented Supply Chain Management: Does Information Sharing help Managers with Service Capacity Decisions? in: Production and Operations Management, Vol. 9, No.1, Spring 2000.

Arlt (Netzwerkflussprobleme, 1994) - Christoph Arlt: Netzwerkflussprobleme. Lösungsansätze unter Berücksichtigung von Fixkosten. Deutscher UniversitätsVerlag, Wiesbaden.

Arthur D. Little (Logistics, 1991) - Arthur D. Little and The University of Pennsylvania, Centre of Logistics Research: Logistics in Service Industries. Prepared for Council of Logistics Management. Oak Brook, Illinois.

Backen et al. (Experimentation, 1992) - B. Backen, J. Gould und D. Kim: Experimentation in learning organizations: A management flight simulator approach. In: European Journal of Operational Research 59 (1).

Bea et al. (ABWL, 2000) - Franz Xaver Bea, Erwin Dichtl und Marcell Schweitzer: Allgemeine Betriebswirtschaftslehre. 8., überarbeitete und erweiterte Auflage, Gustav Fischer Verlag, Stuttgart.

Beer (Cybernetics, 1959) - Stafford Beer: What has Cybernetics to do with Operational Research. Operational Research Quarterly 10 (1959).

Bleicher (Unternehmungsspiele, 1966) - Knut Bleicher: Unternehmungsspiele als Erkenntnismittel für Ausbildung und Forschung. In: Führung in der Wirtschaft (ohne Hrsg.). Festschrift zum zehnjährigen Bestehen der Akademie für Führungskräfte der Wirtschaft (1956 - 1966). Bad Harzburg. 
Bloech / Ihde (Distributionsplanung, 1972) - Jürgen Bloech und Gösta-B. Ihde: Betriebliche Distributionsplanung. Zur Optimierung der logistischen Prozesse. Physica-Verlag, Würzburg und Wien.

Bloech / Rottenbacher (Materialwirtschaft, 1986) - Jürgen Bloech und Stefan Rottenbacher (Hrsg. im Auftrag der Schmalenbachgesellschaft): Materialwirtschaft. C. E. Poeschel Verlag, Stuttgart.

Bloech et al. (Konzernlogistik, 1994) - Jürgen Bloech, Ronald Bogaschewsky, Werner Frank (Hrsg.): Konzernlogistik und Rationalisierungsgemeinschaften mit Lieferanten. Schäffer-Poeschel Verlag, Stuttgart.

Bloech et al. (Ausbildung, 1996) - Jürgen Bloech, Gerald Kauer und Christian Orth: Unternehmensplanspiele in der kaufmännischen Ausbildung, Untersuchungen zum Wissenserwerb. In: Zeitschrift für Berufs- und Wirtschaftspädagogik, Beiheft 13. Franz Steiner Verlag, Stuttgart.

Bloech et al. (Produktion, 1998) - Jürgen Bloech, Ronald Bogaschewsky, Uwe Götze und Folker Roland - Einführung in die Produktion, 3., durchgesehene Auflage. Physica-Verlag, Heidelberg.

Bloech et al. (Weiterbildung, 1998) - Jürgen Bloech, Steffen R. Döhne, Susanne Hartung und Christian Orth: Einsatz eines Planspiels in der kaufmännischen Weiterbildung - Verschiedene Arten der Komplexitätssteigerung im Planspiel. Arbeitsbericht 1/1998, Institut für Betriebswirtschaftliche Produktions- und Investitionsforschung, Abteilung für Unternehmensplanung, Universität Göttingen.

Bloech (Optimalplanung, 1999): Jürgen Bloech - Vorlesungsskriptum Managementlehre und Optimalplanung. Institut für betriebswirtschaftliche Produktions- und Investitionsforschung, Universität Göttingen.

Bloech / Ihde (Logistiklexikon, 1999) - Jürgen Bloech und Gösta B. Ihde (Hrsg.): Vahlens Grosses Logistiklexikon. Verlage Beck / Vahlen, München.

Bloech (Produktionsplanung, 1999) - Jürgen Bloech: Produktionsplanung in Unternehmensplanspielen. In: Kurt Nagel, Roland F. Erben und Frank T. Piller (Hrsg.): Produktionswirtschaft 2000. Perspektiven für die Fabrik der Zukunft. Gabler Verlag, Wiesbaden.

Bowersox (Logistical Management, 1978) - Donald J. Bowersox: Logistical Management: a systems integration of physical distribution management and materials management. Second edition, Macmillan Publishing Co., Inc. New York.

Bowersox et al. (Logistical Excellence, 1992) - Donald J. Bowersox, Patricia J. Daugherty, Cornelia L. Dröge, Richard N. Germain und Dale S. Rogers: Logistical Excellence: it's not business as usual. Digital Equipment Corporation, digital press, Burlington, MA.

Brede (Verwaltungsentscheidungen, 1968) - Helmut Brede: Die wirtschaftliche Beurteilung von Verwaltungsentscheidungen in der Unternehmung. Westdeutscher Verlag, Köln und Opladen.

Brockhaus (LexiROM, 1995) - CD-ROM. Copyright Microsoft Corporation und Bibliographisches Institut \& F.A. Brockhaus AG. 
Bronner / Kollmannsperger (Planspieleinsatz, 1997) - Rolf Bronner und Martina Kollmansperger: Planspieleinsatz an deutschen Hochschulen. In: Zeitschrift für Planung, 8. Jahrgang, Nr. 4.

BO-Cash (Spielanleitung, 2000) - Planspielunterlagen und (Test-) CD-ROM zum Planspiel BO-Cash. logic systems GmbH, Bochum.

Bundeswehr (Logistik, 1982): Logistisches Handbuch der Bundeswehr, Teil 1. Herausgegeben durch den Bundesminister der Verteidigung. Bonn.

Bundeswehr (ZDv 30/41, 1984): Zentrale Dienstvorschrift ZDv 30/41, Begriffe der Logistik und Rüstung. Herausgegeben durch den Bundesminister der Verteidigung. Bonn.

Busch (Leistung, 1998) - Friedhelm Busch: Kosten senken bei höherer Leistung Modernisierungen in der Logistikkette. In: Logistik Jahrbuch 1998. Verlagsgruppe Handelsblatt, Düsseldorf.

Ceppi (Management Games, 1970) - C. Ceppi: Erfahrungen mit Management Games. In: Industrielle Organisation, Heft 7.

Drössler (Planspiele, 1998) - Christoph Drössler: Virtueller Bankrott. Mit Planspielen sollen Manager lernen, ein Unternehmen zu führen. In: Die Zeit. Hamburg, 8. April 1998.

Engel (Konzernlogistik, 1994) - Ulrich Engel: In: Jürgen Bloech, Ronald Bogaschewsky und Werner Frank (Hrsg.): Konzernlogistik und Rationalisierungsgemeinschaften mit Lieferanten. Schäffer-Poeschel Verlag, Stuttgart.

Epus (Spielanleitung, 1992) - Jürgen Bloech und Herbert Rüscher: Spielanleitung EpUS

- Ein-Platz-Unternehmens-Simulation. Institut für Betriebswirtschaftliche Produktions- und Investitionsforschung, Universität Göttingen.

Eversheim / Schuh (Betriebshütte,1996) - Walter Eversheim und Günther Schuh (Hrsg.): Produktion und Management »Betriebshütte«. 7., völlig neu bearbeitete Auflage. Springer Verlag, Berlin, Heidelberg und New York.

FAZ (19. August 2000): Sonderbeilage Internationale Märkte. Frankfurt am Main.

FAZ (30. August 2000): Milliarden-Auftrag für Danzas von der Telekom. Frankfurt am Main.

FAZ (19. September 2000): 81 Prozent der Güter werden auf der Straße transportiert. Frankfurt am Main.

FAZ (9. Oktober 2000): Auch Fonds setzen auf das „Rückgrat der New Economy“. Frankfurt am Main.

Femppel (Kundenlogistik, 1998) - Peter Femppel: Kunden-Logistik in Zeitsprung. In: Logistik Jahrbuch 1998. Verlagsgruppe Handelsblatt, Düsseldorf.

Fiege (Dienstleister, 1998) - Heinz Fiege: Komplexitätsreduktion durch Einschalten logistischer Dienstleister. In: Logistik Jahrbuch 1998. Verlagsgruppe Handelsblatt, Düsseldorf.

Goertzen (Simultanplanung, 1992) - Hannelore Goertzen: Simultanplanung von Produktion und Beschaffung bei substitutionalen Produktionsfaktoren - Eine Entscheidungshilfe im Planspiel SUBPRO. Physica-Verlag, Heidelberg. 
Götze / Mikus (Strategisches Management, 1999) - Uwe Götze und Barbara Mikus: Strategisches Management. GUC - Verlag der Gesellschaft für Unternehmensrechnung und Controlling m.b.H., Chemnitz.

Göpfert et al. (Beschaffungslogistik, 1999) - Ingrid Göpfert, Axel Neher und KlausPeter Jung: Zukünftige Entwicklung der Beschaffungslogistik in der Automobilindustrie. Ergebnisse einer empirischen Untersuchung. In: Logistik Jahrbuch 1999. Verlagsgruppe Handelsblatt, Düsseldorf.

Günther / Kruschwitz (Unternehmungsplanspiel, 1975) - Horst Günther und Lutz Kruschwitz: FINIS - Ein Unternehmungsplanspiel zur Investitions- und Finanzplanung. Erich Schmidt Verlag, Berlin.

Harlacher (Logistikkostenrechnung, 1988) - Klaus Harlacher: Logistikkosten- und Logistikleistungsrechnung der Verkehrsbetriebe der chemischen Industrie. In: Thomas Reichmann (Hrsg.): Controlling-Praxis, Erfolgsorientierte Unternehmenssteuerung. Verlag Franz Vahlen München.

Hartung (Fortbildung, 2000) - Susanne Hartung: Förderung der Lerneffizienz beim Einsatz von Unternehmensplanspielen in der kaufmännischen Fortbildung berufstätiger Erwachsener. Wissenschaftliche Publikationen Duehrenkop \& Radicke, Göttingen.

Heidenberger et al. (F\&E-Unternehmensspiel, 2001) - Kurt Heidenberger, Wolfgang Ristl und Christian Stummer: Planung von Forschungs- und Entwicklungsinvestitionen im Unternehmensspiel. Demnächst veröffentlicht in: Zeitschrift für Planung, 12. Jahrgang. Physica-Verlag, Heidelberg.

Hesse / Schrader (Bewerbungsstrategien, 1995) - Jürgen Hesse und Hans Christian Schrader: Bewerbungsstrategien für Führungskräfte in Industrie, Handel und Öffentlichem Dienst. Fischer Taschenbuch Verlag, Frankfurt am Main.

Hessenberger / Kuhn (KVP, 1996) - Manfred Hessenberger und Joachim Kuhn: KVP: Mit guter Logistik fängt alles an. In: Harvard Business Manager 3/96, Seiten 17 bis 24 .

Hoffmann (Wertanalyse, 1994) - Heinz J. Hoffmann: Wertanalyse, Die westliche Antwort auf KAIZEN. Ullstein Verlag Frankfurt am Main und Berlin.

Hoitsch (Kostenrechnung, 1996) - Hans-Jörg Hoitsch: Kosten- und Erlösrechnung. Eine controllingorientierte Einführung. 2. Auflage, Springer Verlag, Berlin, Heidelberg und New York.

Hug (Beständecontrolling, 1988) - Werner Hug: Ersatzteil-Beständecontrolling, dargestellt am Beispiel eines Unternehmens der Stahlindustrie. In: Thomas Reichmann (Hrsg.): Controlling-Praxis, Erfolgsorientierte Unternehmenssteuerung. Verlag Franz Vahlen München.

Ihde (Transport, 1991) - Gösta B. Ihde: Transport, Verkehr, Logistik. 2., völlig überarbeitete und erweiterte Auflage, Verlag Franz Vahlen, München.

Ischebeck (Unternehmensspiele, 1969) - Wolfram Ischebeck: Unternehmensspiele Neue Möglichkeiten der Weiterbildung. In: Wissenschaft für die Praxis. Heft 10. 
Jünemann (Materialfluss, 1989) - Reinhardt Jünemann: Materialfluss und Logistik: Systemische Grundlagen mit Praxisbeispielen. Springer-Verlag, Berlin und Heidelberg.

Katzmarzyk (Einkaufscontrolling, 1988) - Johannes Katzmarzyk: Einkaufs-Controlling in der Industrie. Bundesverband Materialwirtschaft und Einkauf e.V., Frankfurt am Main.

Kern (Operations Research, 1966) - Werner Kern: Operations Research. 2. Auflage. C. E. Poeschel Verlag, Stuttgart.

Kiesel (Logistik, 1999) - Jens Kiesel: Fachwörter der Logistik, Logistics Dictionary. Herausgegeben von der Siemens AG. 11., wesentlich überarbeitete und erweiterte Auflage. Publicis MCD Verlag, Erlangen und München.

Kleeberg (Transportnetzwerke, 2000) - Lars Kleeberg: Management von Transportnetzwerken. Wissenschaftliche Publikationen Duehrenkop \& Radicke, Göttingen.

Klimaforte (Spielanleitung, 1998) - Manfred Meyer und Martin Schwandt: Spielanleitung KLIMA ${ }^{\text {FORTE }}$ - Klinikmanagement mit Fallpauschalen-orientierter Finanzierung. Forschungsgruppe Medizinökonomie am Lehrstuhl für Betriebswirtschaftslehre und Operations Research der Universität ErlangenNürnberg.

Klöpper (Management, 1991) - Heinz-Jürgen Klöpper: Logistikorientiertes strategisches Management. Erfolgspotentiale im Wettbewerb. Verlag TÜV Rheinland.

Knüwer (Hochschulen, 1998) - Thomas Knüwer: Hochschulen machen das Management spielend einfach. In: Handelsblatt. Düsseldorf, 15. Juni 1998.

Koller (Planspieltechnik, 1969) - Horst Koller: Simulation und Planspieltechnik. Berechnungsexperimente in der Betriebswirtschaft. Gabler Verlag, Wiesbaden.

Koppelmann (Verpackung, 1979) - Udo Koppelmann: Stichwort „Verpackung“. In: W. Kern (Hrsg.): Handwörterbuch der Produktionswirtschaft. Stuttgart.

KPMG (Rechnungslegung, 1999) - KPMG Aktiengesellschaft: Rechnungslegung nach US-amerikanischen Grundsätzen. Grundlagen der US-GAAP und SECVorschriften. 2., überarbeitete und erweiterte Auflage, Berlin.

Kuhn (Unternehmensplanung, 1998) - Axel Kuhn: Unternehmensplanung. In: Sonderbeilage Transfer, Management Berater, Februar 1998.

Küting / Weber (Bilanzanalyse, 1993) - Karlheinz Küting und Claus-Peter Weber: Die Bilanzanalyse. Lehrbuch zur Beurteilung von Einzel- und Konzernabschlüssen. Schäffer-Poeschel Verlag Stuttgart.

Lucke (Information, 1998) - Hans-Joachim Lucke: Jederzeit aktuell informieren. In: Logistik Jahrbuch 1998. Verlagsgruppe Handelsblatt, Düsseldorf.

Mandl et al. (Wissen, 1993) - Heinz Mandl, Hans Gruber und Alexander Renkl: Das träge Wissen. In: Psychologie heute, Heft 9.

Marga (Spielanleitung, 1998): Spielanleitung MARGA - Das General Management Unternehmensplanspiel. Universitätsseminar der Wirtschaft, Schloss Gracht. 
Merkel (Managementsysteme, 1995) - Helmut Merkel: Logistik Managementsysteme, Grundlagen und informationstechnische Umsetzung. R. Oldenbourg Verlag, München und Wien.

Meyer (Systemforschung, 1995) - Manfred Meyer: Operations Research. Systemforschung. 4. überarbeitete Auflage, Gustav Fischer Verlag, Stuttgart.

Meyer / Hansen (Planungsverfahren, 1996) - Manfred Meyer und Klaus Hansen: Planungsverfahren des Operations Research. 4., völlig überarbeitete und ergänztre Auflage. Verlag Franz Vahlen, München.

Meyers (Lexikon, 1977): Meyers Enzyklopädisches Lexikon, Band 20. Bibliographisches Institut, Mannheim.

Miebach / Blauermel (Beschaffungszentrum, 1998) - Joachim Miebach und Gregor Blauermel: Kooperation im Beschaffungszentrum. In: Logistik Jahrbuch 1998. Verlagsgruppe Handelsblatt, Düsseldorf.

Milling (Modeling, 1996) - Peter M. Milling: Modeling innovation processes for decision support and management simulation. in: System Dynamics Review, Volume 12, No. 3.

Nagel (Just-in-Time, 1993) - Bernhard Nagel: Just-in-Time-Produktion und Lieferantenstellung. In: Peter Milling und Günther Zäpfel (Hrsg.): Betriebswirtschaftliche Grundlagen moderner Produktionsstrukturen. Verlag Neue Wirtschafts-Briefe, Herne und Berlin.

Neff / Citrin (Lessons, 2000) - Thomas J. Neff und James M. Citrin: Lessons from the Top. In search of the best Business Leaders. Penguin Books Ltd., Harmondsworth.

Orth (Planspiele, 1999) - Christian Orth: Unternehmensplanspiele in der betriebswirtschaftlichen Aus- und Weiterbildung. Josef Eul Verlag, Lohmar und Köln.

Otto et al. (Nadelöhr, 1998) - Jürgen Otto, Matthias Zeller und Michael Müller-Berg: Die Daten im Nadelöhr. In: Logistik Jahrbuch 1998. Verlagsgruppe Handelsblatt, Düsseldorf.

Pack (Planspiele, 1968) - Ludwig Pack: Unternehmer-Planspiele für die betriebswirtschaftliche Ausbildung, Spielebeschreibungen. Westdeutscher Verlag, Köln und Opladen.

Paich / Sterman (Experimental Markets, 1993) - Mark Paich und John D. Sterman: Boom, bust, and failures to learn in experimental markets. In: Management Science Vol. 39, No. 12.

Pawellek / Hartman (Produktionslogistik, 1999) - Günther Pawellek und Thorsten Hartmann: Komplexitätsreduzierung in der Produktionslogistik. In: Logistik Jahrbuch 1999. Verlagsgruppe Handelsblatt, Düsseldorf.

Pederson (Simulation, 2000) - Paul B. Pederson: One in the eye is worth two in the ear! In: Simulation and Gaming Vol. 31, No. 1.

Penta (Spielanleitung, 1991) - Jürgen Bloech, Herbert Rüscher und Uwe Schubert: Spielanleitung PENTA - Planspiel Entsorgungs- und Abfallwirtschaft. Institut 
für betriebswirtschaftliche Produktions- und Investitionsforschung, Universität Göttingen.

Pfohl (Logistiksysteme, 2000) - Hans-Christian Pfohl: Logistiksysteme Betriebswirtschaftliche Grundlagen. 6. neubearbeitete und aktualisierte Auflage, Springer Verlag, Berlin, Heidelberg und New York.

Pfohl / Schäfer (Beschaffungsprozess, 1999) - Hans-Christian Pfohl und Christian Schäfer: Entscheidungen im Beschaffungsprozess. In: Logistik Jahrbuch 1999. Verlagsgruppe Handelsblatt, Düsseldorf.

Porter (Wettbewerbsvorteile, 1992) - Michael E. Porter: Wettbewerbsvorteile (Competitive Advantages). Spitzenleistungen erreichen und behaupten. 3. Auflage, Campus Verlag, Frankfurt am Main

Prehm (Marketingplanspiel, 1995) - Hans-Jürgen Prehm: Marketing-Unternehmensspiel MARKUS - Modelldarstellung und Instrumente zur Entscheidungsvorbereitung. Deutscher Universitäts-Verlag, Wiesbaden.

Prehm / Ehlken (Planspiel, 1995) - Hans-Jürgen Prehm und Jörg Ehlken: Unternehmensspiel ISOS - Simulation internationaler Standorte und Märkte. Arbeitsbericht 2/1995, Institut für Betriebswirtschaftliche Produktions- und Investitionsforschung, Abteilung für Unternehmensplanung, Universität Göttingen.

Protil (Materiallogistik, 1997) - Roberto Max Protil: Effizienz- und Risikoanalyse der Materiallogistik in landwirtschaftlichen Genossenschaften anhand eines Simulationsmodells. Cuvillier Verlag, Göttingen.

Puma (Spielanleitung, 1992) - Jürgen Bloech, Herbert Rüscher und Martin Dick: Spielanleitung PUMA - Produktions- und Marketing-Simulation. Institut für betriebswirtschaftliche Produktions- und Investitionsforschung, Universität Göttingen.

Reutner (Strategietagung, 1995) - Friedrich Reutner: Die Strategietagung. 2. Auflage, Gabler Verlag, Wiesbaden.

Ritter (Verpackungsoptimierung, 1980) - Thomas Ritter: Betriebswirtschaftliche Verpackungsoptimierung, Überlegungen zur zieladäquaten Konzeption von Verpackungslösungen. Eichhorn-Verlag, Göttingen.

Rohn (Unternehmensplanspiel, 1964) - Walter E. Rohn: Führungsentscheidungen im Unternehmensplanspiel. Veröffentlichung des Deutschen Instituts zur Förderung des industriellen Führungsnachwuchses, Band 6, Essen.

Schenk / Jahn (Recycling, 1997) - Michael Schenk und Carlos Jahn: Integration des Recyclings in die Produktion - Herausforderungen für Planung und Logistik. In: Karl Inderfurth, Michael Schenk und Dietrich Ziems (Hrsg.): Logistik auf Umweltkurs: Chancen und Herausforderungen. 3. Magdeburger Logistiktagung, 20. - 21. November 1997. Otto-von-Guericke-Universität Magdeburg.

Schierenbeck (BWL, 2000) - Henner Schierenbeck: Grundzüge der Betriebswirtschaftslehre. 15., überarbeitete und erweiterte Auflage. R. Oldenbourg Verlag, München und Wien. 
Schöl (Modellentwurf, 1998) - Klaus Schöl: Konzeption handelsbetrieblicher Unternehmenssimulationen und Planspiele. Ein Modellentwurf für den mittelständischen Textileinzelhandel. Dissertation, Universität Göttingen.

Schöllhammer (Planspiele, 1964) - Hans Schöllhammer: Bedeutung von nichtmaschinengebundenen Unternehmungsspielen als Methode zur Ausbildung von Führungskräften. In: Betriebswirtschaftliche Forschung und Praxis 6/1964.

Schulte (Logistik, 1995) - Christof Schulte: Logistik - Wege zur Optimierung des Material- und Informationsflusses. 2., überarbeitete und erweiterte Auflage, Verlag Franz Vahlen, München.

Schwandt (Planspiel, 1998) - Martin Schwandt: Entwicklung eines Planspiels zum Klinikmanagement mit Fallpauschalen-orientierter Finanzierung: KLIMA ${ }^{\text {FORTE }}$. Forschungsgruppe Medizinökonomie am Lehrstuhl für Betriebswirtschaftslehre und Operations Research der Universität Erlangen-Nürnberg.

Severin (Warenumschlag, 1998) - Hans Gerd Severin: Der automatische Warenumschlag wird Realität. In: Logistik Jahrbuch 1998. Verlagsgruppe Handelsblatt, Düsseldorf.

Simlog (Spielanleitung, 1998) - Jürgen Bloech und Herbert Rüscher: Unterlagen und Skriptum zu SIM-LOG - Simulation zur Logistik. Institut für betriebswirtschaftliche Produktions- und Investitionsforschung, Universität Göttingen.

Staufenbiel (Berufsplanung, 2000) - Joerg E. Staufenbiel: Berufsplanung für den Managementnachwuchs. 20. Auflage, Staufenbiel Institut, Köln.

Strauß (Controlling, 1993) - Peter Strauß: Controlling als notwendiges Führungsinstrument für den modernen Produktionsbetrieb. In: Peter Milling und Günther Zäpfel (Hrsg.): Betriebswirtschaftliche Grundlagen moderner Produktionsstrukturen. Verlag Neue Wirtschafts-Briefe, Herne und Berlin.

Süddeutsche Zeitung (17./18. Juni 2000): Was macht eigentlich ein Supply Chain Manager? München.

Süddeutsche Zeitung (20. September 2000): Stellenanzeige der Volkswagen AG. München.

Verein Deutscher Ingenieure (VDI-Richtlinie 3633, 1996): Hrsg. vom Verein Deutscher Ingenieure e.V.. Düsseldorf.

Wäscher (Layout, 1993) - Gerhard Wäscher: Logistikorientiertes Layout von Fertigungssystemen. In: Peter Milling und Günther Zäpfel (Hrsg.): Betriebswirtschaftliche Grundlagen moderner Produktionsstrukturen. Verlag Neue Wirtschafts-Briefe, Herne und Berlin.

Weber (Logistik-Controlling, 1990) - Jürgen Weber: Logistik-Controlling. C. E. Poeschel Verlag, Stuttgart.

Wehking / Schulz (Fahrzeugkonzepte, 1999) - Karl-Heinz Wehking und Robert Schulz: Systematische Entwicklung innovativer Fahrzeugkonzepte für die zukünftigen Aufgaben der Entsorgungswirtschaft. In: Logistik Jahrbuch 1999. Verlagsgruppe Handelsblatt, Düsseldorf. 
Wildemann (Logistik, 1992) - Horst Wildemann: Logistik. In: Karlheinz Küting und Axel Schnorbus (Hrsg.): Betriebswirtschaftslehre heute. Für die Aufgaben der Praxis. Verlag Frankfurter Allgemeine Zeitung, Frankfurt am Main.

Wilken (Qualität, 1993) - Carsten Wilken: Strategische Qualitätsplanung und Qualitätskostenanalysen im Rahmen eines Total Quality Management. PhysicaVerlag, Heidelberg.

Wöhe (Bilanzierung, 1997) - Günter Wöhe: Bilanzierung und Bilanzpolitik. 9. völlig überarbeitete Auflage, Verlag Franz Vahlen, München.

Wöhe (ABWL, 2000) - Günter Wöhe: Einführung in die Allgemeine Betriebswirtschaftslehre. 20., neubearbeitete Auflage. Verlag Franz Vahlen, München.

Wöhe / Bilstein (Unternehmensfinanzierung, 1988) - Günter Wöhe und Jürgen Bilstein: Grundzüge der Unternehmensfinanzierung. 5., überarbeitete Auflage. Verlag Franz Vahlen, München.

Woll (AVWL, 2000) - Artur Woll: Allgemeine Volkswirtschaftslehre. 13., überarbeitete und ergänzte Auflage. Verlag Franz Vahlen, München

Wundrack (Vertriebskostenrechnung, 2000) - Carsten Wundrack: Industrielle Vertriebskostenrechnung. Josef Eul Verlag, Lohmar und Köln.

Zäpfel (Produktionsplanung, 1993) - Günther Zäpfel: Produktionsplanung und -steuerung in der „Fabrik der Zukunft“. In: Peter Milling und Günther Zäpfel (Hrsg.): Betriebswirtschaftliche Grundlagen moderner Produktionsstrukturen. Verlag Neue Wirtschafts-Briefe, Herne und Berlin. 


\section{Versicherung an Eides Statt}

„Ich versichere an Eides Statt, dass ich die eingereichte Dissertation

\section{Logistische Entscheidungen und ihre Auswirkungen: \\ Die Unternehmenssimulation LogisticPLUS}

selbständig verfasst habe. Anderer als der von mir vorgegebenen Hilfsmittel und Schriften habe ich mich nicht bedient. Alle wörtlich oder sinngemäß den Schriften anderer Autoren entnommenen Stellen habe ich kenntlich gemacht."

Mannheim, im November 2000

Maximilian Eichhorn 


\section{Autor}

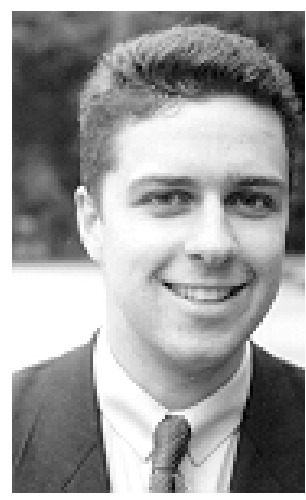

Persönliches

Schule

Studium

Berufserfahrung

Militär

\section{Maximilian Eichhorn}

\section{St.-Veit-Str. 43}

81673 München

m.eichhorn@LogisticPLUS.de

geboren am 8. Januar 1969 in Münster/Westfalen, verheiratet mit Susanne Eichhorn geb. Jäger

Grundschule und altsprachliches Gymnasium am Kaiserdom in Speyer, drei Monate Europäische Schule in Culham / Oxford Abitur 1988

1990 bis 1993: Elektrotechnik (TU München, Uni Jena)

1993 bis 1994: Wirtschaftsmathematik (Uni Ulm, Vordiplom Wirtschaftswissenschaften)

1994 bis 1997: Elektrotechnik (TU Wien, Diplom-Ingenieur)

1998 bis 2000: Promotionsstudium Betriebswirtschaftslehre (Uni Göttingen, Dr.rer.pol.)

während des Studiums neun Praktika, davon drei im Ausland

März 1998 bis August 2000: Assistent des Vorstands, später Produkt Manager, Friatec AG, Mannheim

Ab September 2000: Unternehmensberater, Siemens Management Consulting, Siemens AG, München

Juli 1988 bis Juni 1990 Militärdienst in der Gebirgsdivision, Ausbildung zum Reserveoffizier, 12 Wehrübungen und Lehrgänge, davon 2 im Ausland (USA, Schweiz), Hauptmann der Reserve 
